BNL--52317-Vol.1

\title{
National Synchrotron Light Source Annual Report 1991 Volume I
}

(For the period of October 1, 1990 through September 30, 1991)

\author{
Editors: \\ S. L. Hulbert and N. M. Lazarz
}

\section{April 1992}

The National Synchrotron Light Source Department is supported by the Office of Basic Energy Sciences United States Department of Energy Washington, D.C.

Brookhaven National Laboratory Associated Universities, Inc. Upton, New York 11973 Under Contract No. DE-AC02-76CH00016 with the United States Department of Energy 


\title{
EDITORS' NOTE
}

The Editors of the 1991 NSLS Annual Report would like to recognize Lydia Lever for her dedicated effort in typing and unending patience in compiling the NSLS Staff and User Publication lists, the latter being a major tangle of differing formats and degrees of completeness (what a mess). We also recognize Lisa Lo Sordo for painstaking typing of the lists of VUV and X-ray abstracts.

This year's Annual Report is divided into two volumes. Volume I contains scientific highlights, operational summaries, and reports from workshops and NSLS projects, while Volume II contains all contributed VUV and X-ray abstracts. By making this division, Volume I becomes a compact summary of FY1991 work conducted at the NSLS, which can be distributed to visitors as well as to NSLS beamlines and users. Volume II will be provided as a reference volume to each NSLS beamline and to the libraries of synchrotron radiation facilities around the world. In an effort to save trees, Volume II will be provided to individuals only upon request (contact NSLS User Administration).

As you will discover, the NSLS FY1991 scientific highlights have been compiled by a collaborative group of NSLS scientific staff members and Special Interest Group representatives, with our thanks. An effort has been made to make these summaries more interesting by including a number of figures with informative captions. These figures include all beamline scientific highlights contributed by users. Beamline operational highlights remain in their own section $(\mathrm{V})$; we thank all contributors and renew our request for greater participation next year (note that the cover photographs are selected from the contributed highlights).

This report was prepared as an acc iunt of work sponsored by an agency of the United States Government. Neither the Uniced States Government nor any agency thereof, nor any of their employees, nor any of their contractors, subcontractors, or their employees, makes any warranty, expres, or implied, or assumes any legal liability or responsibility for the accuracy, complateness, or usefulness of any information, apparatus, product, or process disclosed, or represents that its use would not infringe privately owned rights. Reference herein to any specific commercial product, process, or service by trade name, trademark, manufacturer, or otherwise, does not necessarily constitute or imply its endorsement, recommendation, or favoring by the United States Government or any agency, contractor or subcontractor thereof. The views and opinions of authors expressed herein do not necessarily state or reflect those of the United States Government or any agency, contractor or subcontractor thereof.

\author{
Printed in the United States of America \\ Available from \\ National Technical Information Service \\ U.S. Department of Commerce \\ 5285 Port Royal Road \\ Spring field, VA 22161 \\ NTIS price codes: \\ Printed Copy: A09; Microfiche Copy: A01
}




\section{TABLE OF CONTENTS}

\section{VOLUME I}

\section{SECTION I \\ INTRODUCTION}

Introduction by the Chairman. ................................... 3

Users' Executive Committee ................................Mark Rivers . ......4 4

Scientific Program Support, Committe $\ldots \ldots \ldots \ldots \ldots \ldots \ldots \ldots$ Randy Alkire/Roger Klaffky . . . . . . 6

\section{SCIENTIFIC DISCIPLINES:}

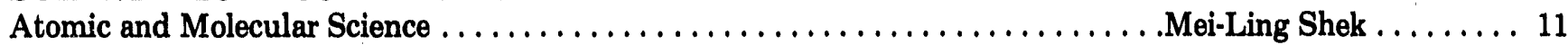

Energy Dispersive Diffraction $\ldots \ldots \ldots \ldots \ldots \ldots \ldots \ldots \ldots \ldots \ldots \ldots \ldots \ldots \ldots$ Earl Skelton $\ldots \ldots \ldots \ldots 17$

Lithography, Microscopy and Tomography ...................Gwyn Williams ...... 20

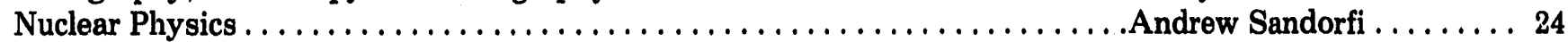

UV Photoemission and Surface Science $\ldots \ldots \ldots \ldots \ldots \ldots \ldots \ldots \ldots \ldots \ldots$ Chi-Chang Kao . . . . . 26

X-ray Absorption Spectroscopy .....................Lars Furenlid/Chuck Bouldin . ...... 32

X-ray Scattering and Crystallography $\ldots \ldots \ldots \ldots \ldots \ldots \ldots \ldots$ Lonny Berman $/$ Peter Siddons $\ldots \ldots \ldots 36$

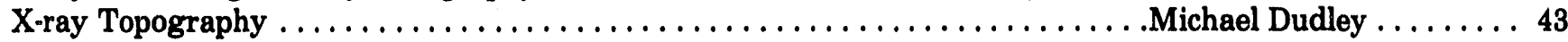

\section{SECTION II}

\section{SYMPOSIA, WORITSHOPS AND PROJECTS}

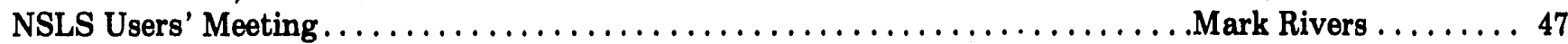

Workshop on Surface Structure $\ldots \ldots \ldots \ldots \ldots \ldots \ldots \ldots \ldots \ldots \ldots \ldots \ldots \ldots$. . . . . . . . . . . . 49

Workshop on Electronic and Chemical Phenomena at Surfaces . . . . . . Kevin Smith/Neal Shinn . . . . . . 50

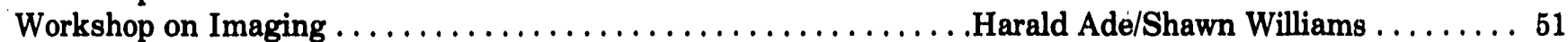

UV FEL Machine Reviews . ........................Sam Krinsky/Ilan Ben-Zvi . . . ... 52

DOE High Schno! Honors Program $\ldots \ldots \ldots \ldots \ldots \ldots \ldots \ldots \ldots \ldots \ldots \ldots$ William Lynch $\ldots \ldots \ldots . \ldots 4$

\section{SECTION III}

\section{FACILITY REPORT}

VUV Machine Operations. ................................Steve Kramer . . . . 59

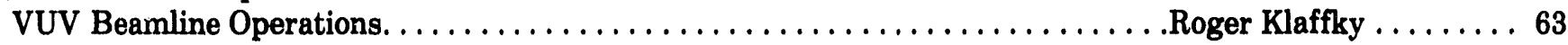

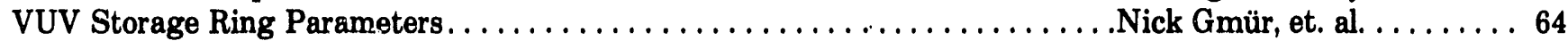

X-ray Machine Operations $\ldots \ldots \ldots \ldots \ldots \ldots \ldots \ldots \ldots \ldots \ldots \ldots \ldots \ldots \ldots \ldots \ldots$ Norman Fewell $\ldots \ldots \ldots . \ldots 6$

X-ray Beamline Operations $\ldots \ldots \ldots \ldots \ldots \ldots \ldots \ldots \ldots \ldots \ldots \ldots \ldots \ldots \ldots$ Roger Klaffky $\ldots \ldots \ldots \ldots 8$

X-ray Storage Ring Parameters $\ldots \ldots \ldots \ldots \ldots \ldots \ldots \ldots \ldots \ldots \ldots \ldots$ Nick Gmür, et. al. . . . . . 70

Users Facilities . . . . . . . . . . . . . . . . . . . . . . . . . . . . . William Thomlinson . . . . . 71

\section{SECTION IV}

\section{NSLS PROJECTS}

Superconducting X-ray Lithography Source (SXLS) $\ldots \ldots \ldots \ldots \ldots \ldots \ldots \ldots$ Richard Heese $\ldots \ldots \ldots 77$

SXLS Storage Ring Parameters $\ldots \ldots \ldots \ldots \ldots \ldots \ldots \ldots \ldots \ldots \ldots$ Jim Murphy, et. al. . . . . . 78

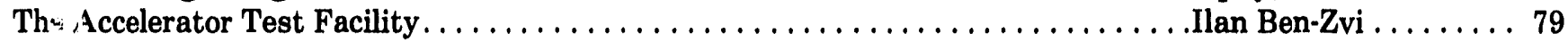

Propused UV-FEL User Facility at the NSLS . . . . . . . . Ilan Ben-Zvi/Sam Krinsky/Li-Hua Yu . . . . . 81

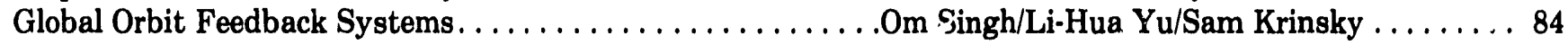

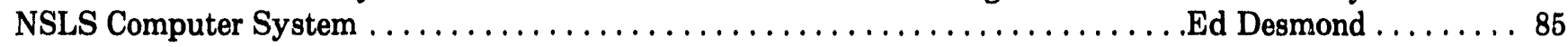

\section{SECTION V}

1991 NSLS OPERATIONAL HIGHLIGHTS

Beamline Operational Highlights . 


\section{SECTION VI}

INFORMATIONAL GUIDE

Informational Guide to the NSLS VUV Beamlines . . . . . . . . . . . . . . . User Administration . . . . . . 105

Informational Guide to the NSLS X-ray Beamlines. . . . . . . . . . . . User Administration . . . . . 109

\section{SECTION VII}

\section{APPENDICES}

List of Abstracts: VUV Beamlines (Abstracts Located in Volume II). . . . . . . . . . Lisa Lo Sordo . . . . . . 119

List of Abstracts: X-ray Beamlines (Abstracts Located in Volume II) . . . . . . . . . Lisa Lo Sordo . . . . . . 127

NSLS Users' Publications $\ldots \ldots \ldots \ldots \ldots \ldots \ldots \ldots \ldots \ldots \ldots \ldots \ldots \ldots$. Lisa Lo Sordo . . . . . . 147

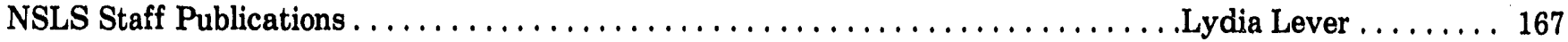

NSLS Staff Preprints $\ldots \ldots \ldots \ldots \ldots \ldots \ldots \ldots \ldots \ldots \ldots \ldots \ldots \ldots \ldots$. $\ldots \ldots \ldots$ ydia Lever $\ldots \ldots \ldots \ldots 173$

NSLS Staff BNL Formal and Informal Reports $\ldots \ldots \ldots \ldots \ldots \ldots \ldots \ldots \ldots$. Lydia Lever . . . . . 175

\section{TABLE OF CONTENTS}

\section{VOLUME II}

\section{SECTION I}

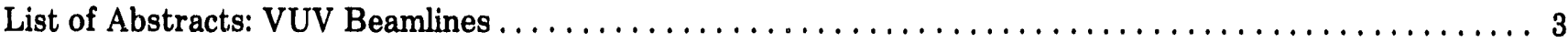

A.bstracts of VUV Beamlines $\ldots \ldots \ldots \ldots \ldots \ldots \ldots \ldots \ldots \ldots \ldots \ldots \ldots \ldots \ldots \ldots \ldots \ldots \ldots \ldots$

\section{SECTION II}

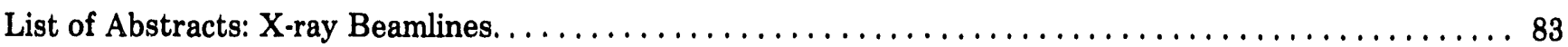

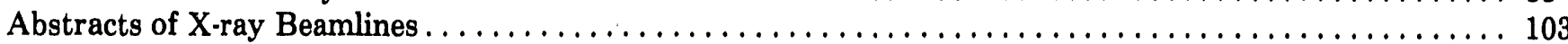




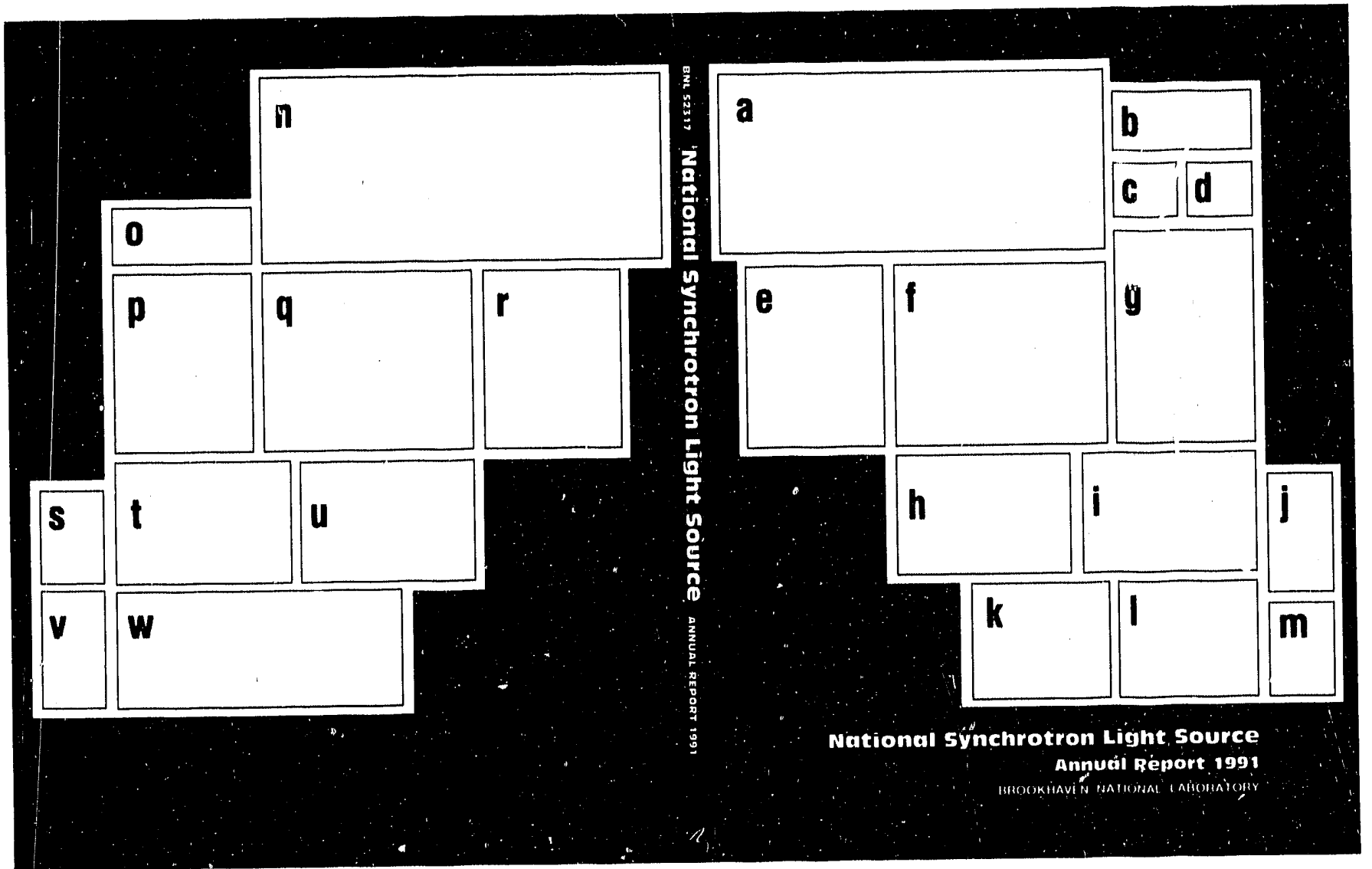

\section{FRONT COVER PHOTOGRAPHS}

a - The National Synchrotron Light Source, Building 725 .

b - Scanning electron miscroscope photos of 0.1 micron lines and spaces in a $60 \mathrm{~nm}$ thick layer of PMMA resist, patterned using 3 different fabrication techniques by the Advanced Lithography Research Department of AT\&T Bell Labs at beamline U13UB. See Section I - Lithography, Microscopy and Tomography.

c - Three $2 \times 2 \mu \mathrm{m}^{2}$ images of an Au test object, as viewed in the X1A Scanning Transmission Electron Microscope (STEM). See Section I - Lithography, Microscopy and Tomography.

d - Copper monolith body of the beam position monitor developed by E. Johnson and T. Oversluizen (NSLS Beamline R \& D Section). See Rev. Sci. Instrum. 60, 1947 (1989).

e - The crystal structure of the superconducting Buckminsterfullerence compound $\mathrm{K}_{3} \mathrm{C}_{60}$. The raspberries are $\mathrm{C}_{60}$ and the smaller isolated spheres are potassium cations. This structure was solved by Peter Stephens and coworkers at SUNY and UCLA, from powder diffraction data taken at beamline X3. See Section I - X-ray Scattering and Crystallography.

f - Andy Warkentien (Technical Specialist in the NSLS Mechanical Group) and John Flannigan (Computer Analyst in the NSLS Computer Controls Group) adjust the liquid helium refrigerator to optimize the performance of the X17 Superconducting Wiggler Cryogenic System. 
g - Movable exit slit chamber for the U13UA beamline, sandwiched between long welded bellows and mounted on a $880 \mathrm{~mm}$-travel linear slide. The distance from the sample chamber to the center of the U13 insertion device is 23 meters. S. L. Hulbert (NSLS, Beamline R \& D Section).

h - The Phase I Superconducting X-ray Lithography Source (SXLS) Storage Ring.

i - VUV front-end fast valves during assembly.

j - Tony Lenhard (NSLS Beamline R \& D Section) inspecting an adjustable crystal holder for a double crystal monochromator. These monochromators are currently installed at X19A, X12B/C and X7.

k - Crystal holders for a double crystal monochromator. T. Oversluizen (NSLS Beamline R \& D Section).

1 - Molecular structure of Buckminsterfullerence, $C_{60}$. D. Cox (BNL Physics) and P. Heiney and coworkers (U. Penn) at beamline X7A have carried out high resolution powder diffraction experiments which revealed the existence of an orientational ordering transition in $\mathrm{C}_{60}$ at $250 \mathrm{~K}$ and an analogous transition in $\mathrm{C}_{70}$. See Section I - X-ray Scattering and Crystallography.

m - Schematic of the water cooled copper monolith body of the NSLS developed beam position monitors. The tungsten blades which are used to sense the beam position are also shown. E. Johnson and T. Oversluizen (NSLS Beamline R \& D Section). See Rev. Sci. Instrum. 60, 1947 (1989).

\section{BACK COVER PHOTOGRAPHS}

$\mathrm{n}$ - The U4IR beamline on the VUV Storage Ring includes three Nicolet interferometers covering the wavelength region from 1 micron to $1 \mathrm{~mm}$. See Section V - Beamline Operational Highlights.

0- A 44 year old male sustained an acute myocardial infarction on Feb. 19, 1991. On Feb. 25, cardiac catherization revealed an $80 \%$ stenosis of the Right Coronary Artery (RCA) - see left hand Cine (film) image. Balloon angioplasty was performed to re-open the RCA - see middle Cine image. Transvenous coronary angiography was performed on Aug. 22, 1991 at the Synchrontron Medical Research Facility (SMERF) showing that the RCA was still patent - see right hand SMERF image, Left Anterior Oblique $40^{\circ}$ view. Beam parameters: X17 superconducting wiggler field was 3.0 Tesla; ring current was $206 \mathrm{~mA}$.

p - Computed microtomography (CMT) image of the internal structure in a $1 \mathrm{~cm}$ fragment of the Allende carbonaceous meteorite. This 2-dimensional image is one of 40 slices in a 3-dimensional tomogram abtained in the superconducting wiggler beamline (X17B1). The high energy wiggler radiation is essential to penetrate these large, dense objects. CMT of meteorites is valuable because of the ability to decermine the positions of the pseudo-circular objects (silicate-bearing chondrules that formed in the early solar nebula) prior to developing sampling strategies and allocation plans. The techniques being de:veloped at X17B1 will be particularly useful in studying rare meteorites collected in Antarctica, some of which come from the Moon and probably Mars. 
q - Developers of the X1A SPEM (left to right) D. Kern, IBM, E. Anderson, LBL, E. Johnson (back), NSLS, J. Kirz, SUNY at Stony Brook, S. Hulbert, NSLS, and H. Ade, SUNY at Stony Brook. H. Ade and J. Kirz were the principal investigators of this collaborative project which received a $1991 \mathrm{R} \& \mathrm{D}$ 100 Award.

$r$ - Using a soft x-ray undulator source and a Fresnel zone plate to produce a focused x-ray probe, researchers at X1A have obtained $50-75 \mathrm{~nm}$ resolution images of the ZnS-based P31 phosphor grains shown here. C. Jacobsen, S. Lindaas, S. Williams, and X. Zhang (SUNY at Stony Brook). See Section I - Lithography, Microscopy and Tomography.

s - Laue crystal used in monochromators.

t - A Couette Shear Cell at beamline X10A is designed for in-situ $\mathrm{x}$-ray scattering of macromolecular fluids under flow. The purpose of this research program is to explore new non-equilibrium steady-state structures of complete fluids that are produced under shear flow, due to the continuous input and dissipation of energy. See Section V - Beamline Operational Highlights.

$\mathrm{u}$ - Beamline U3A during re-assembly. The large chamber at right-center houses the new monochromator. Two of three calibration chambers are located left (downstream) of the monochromator. See Section $\mathrm{V}$ - Beamline Operational Highlights.

v - X-ray scattering at beamline X22C. Upper figure: The diffraction pattern obtained for $\mathrm{Pt}(111)$ at high temperatures in the reconsiructed phase. Lower figure: "ring scans" taken around the $(1,0)$ reflection as a function of temperature. A. R. Sandy, S. G. J. Mochrie (MIT), D. M. Zehner (ORNL), G. Grübel, K. G. Huang, and D. Gibbs (BNL Physics). See Section I - X-ray Scattering and Crystallography.

w - Two X1A-SPEM images of a microfabricated device, acquired simultaneously by monitoring the sample current (representative of the sum of all electrons librated) together with the intensity of the electron energy analyzer. D. Kern, IBM, E. Anderson, LBL, E. Johnson, NSLS, J. Kirz, SUNY at Stony Brook, S. Hulbert, NSLS, and H. Ade, SUNY at Stony Brook. See Section I - Lithography, Microscopy and Tomography. 

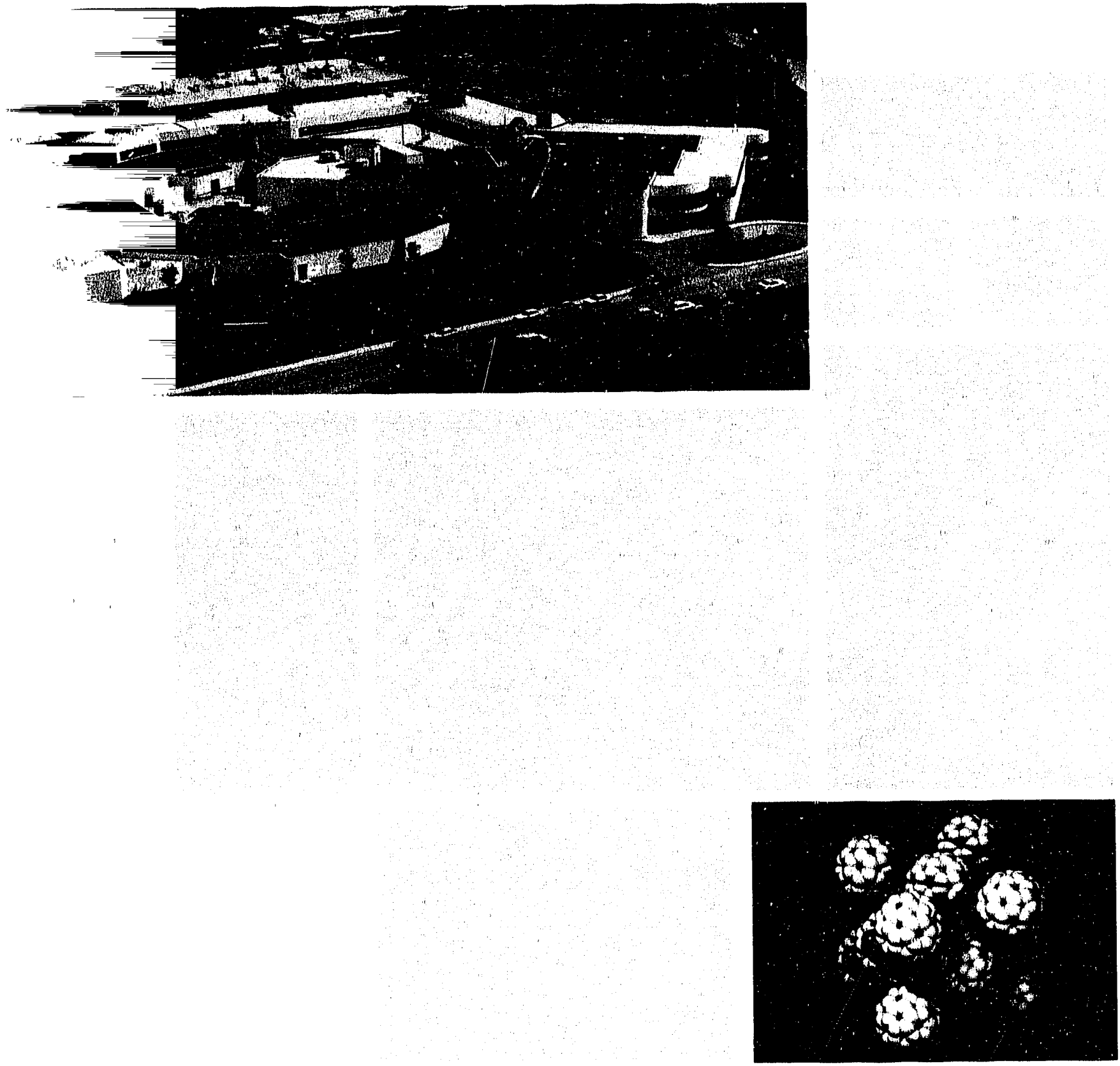

Section I Introduction 


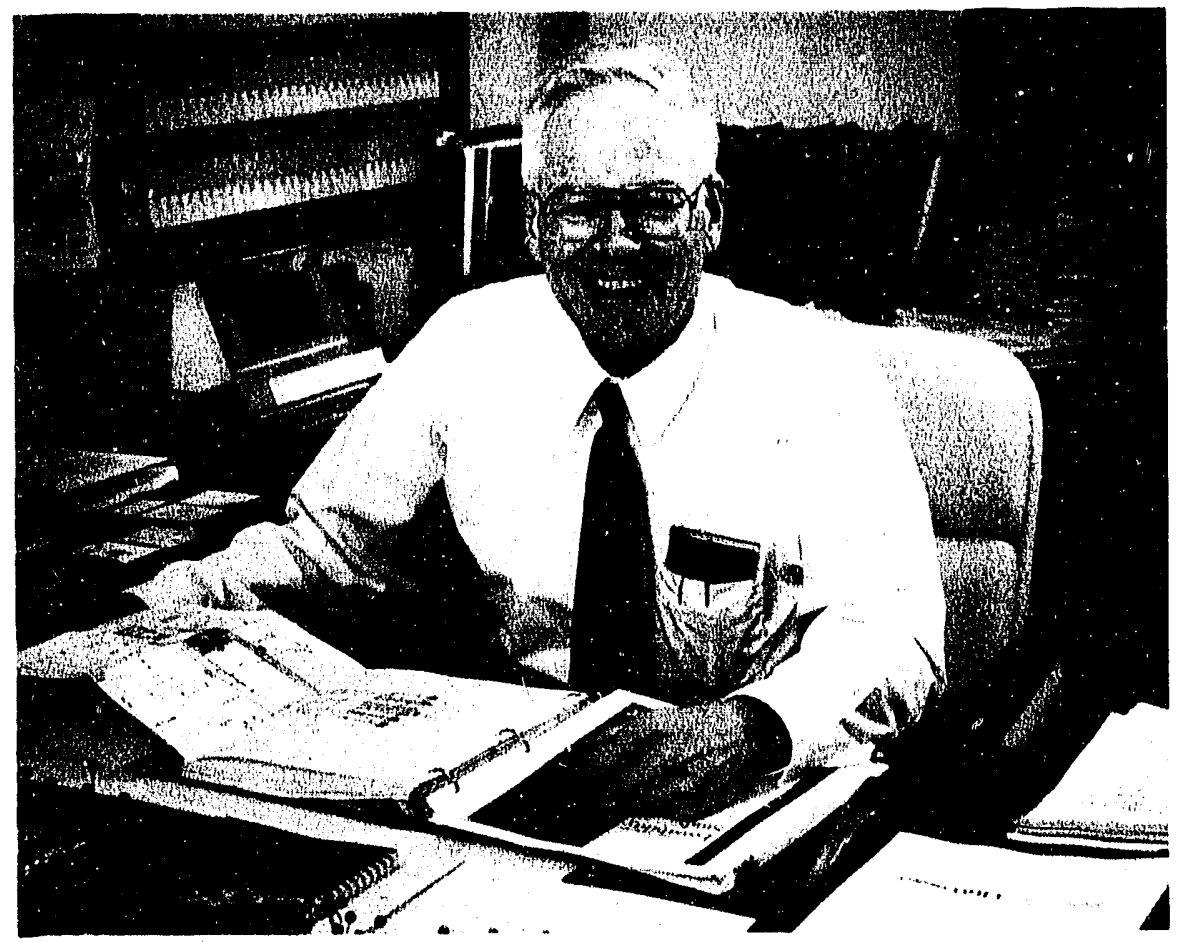

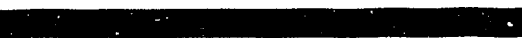

\section{INTRODUCTION}

\section{Denis McWhan \\ NSLS Chairman}

The National Synchrotron Light Source is probably the largest user facility in the world with 3000 users from 400 institutions. The number of users continues to grow with no sign of saturating, and the number of publications based on work done at the NSLS during FY91 was 435 . There is more than one Physical Review Letter published each week which is based on synchrotron radiation and approximately $60 \%$ of these come from the NSLS. This only represents one of the many fields in which forefront research is done at the Light Source. The Friday lunch seminars feature two informal 20 minute talks of new things being done on the X-ray and VUV Rings and attract an audience of close to 50 people. It is impossible to walk around the flool and not find something new and exciting that is being done.

The impressive improvement in orbit stability is clearly the highlight of this years accelerator phyeics program. The real time, global harmonic feedback is operating routinely on both the X-ray and VUV Rings in the vertical and on the X-ray Ring in the horizontal. The vertical orbit is stable to better than 20 microns during a fill, and this has made possible new classes of experiments. A 4th harmonic cavity is operating in the VUV Ring and tuned so as to double the length of the electron bunch. This results in a doubling of the lifetime on the VUV Ring. A third klystron was added to the linac so as to increase the injection energy to $130 \mathrm{MeV}$, and a major program is underway to improve the operation of the booster. The controls group has made major strides in understanding and dramatically improving the communications rate between the micros and the workstations. Implementing Ethernet communications will lead to improved reliability and performance. Studies of the water system have led to an understanding of its deficencies, and a plan to improve the stability of the water temperature is underway.

Major improvements have been made in the work environment for the users of the NSLS. A rearrangement of various groups and facilities has led to a consolidation of user services. A new user administration office is located right upstairs from the front door, and the new electronic registration system painlessly takes care of all the new users. We have established a permanent memorial to the accelerator physicists whose work forms the basis of all low emittance storage rings with the Chasman-Green Memorial Library/Lounge which is adjacent to the experimental floor. The library/lounge provides a place to discuss new results, to consult a reference book or to take a nap, and should provide a focus of activity on the floor. Adjacent to the library are the computerized stockroom, the offices of the flow. manager, control room supervisors, safety engineers, and safety and environmental personnel, and the canteen/kitchen. Most of the people and facilities that the users need are now located in one area. The user shop has been improved and is now staffed during working hours. More staff has been added to keep the facility beamlines at a 
state of the art level and to develop new techniques. The Directorate of Brookhaven National Laboratory has shown its strong commitment to the NSLS by providing money to expand the build. ing between $\mathrm{X} 1$ and $\mathrm{X} 5$ on the $X$-ray Ring. We are optimistic that line item funding will be obtained this year for an addition devoted to structural biology research between X5 and X11. With these additions we will come close to being able to provide secure setup space adjacent to the experimental floor for each Par. ticipating Research Team.

The superconducting x-ray lithography source (SXLS) is moving forward. The Phase I project in. volved building a storage ring with conventional iron magnets. This ring was commissioned and extensive accelerator physics studies have been done during the year. The ring has stored currents of 1.5 Amperes, which is three times the design specification. Construction of the Phase II ring, which will use superconductir.g 180 degree dipole magnets, is underway.

The design of a Free Electron Laser user facility, operating in the ultra-violet, has continued and has been extensively reviewed by a blue ribbon FEL panel. Preconstruction R\&D, suggested by the panel, is beginning at the Accelerator Test Facility with collaborations involving the NSLS and Chemistry departments at BNL and the Grumman Aerospace Company. We look forward to important accelerator physics developments being made using the X-ray and VUV Rings, the SXLS, and the ATF.

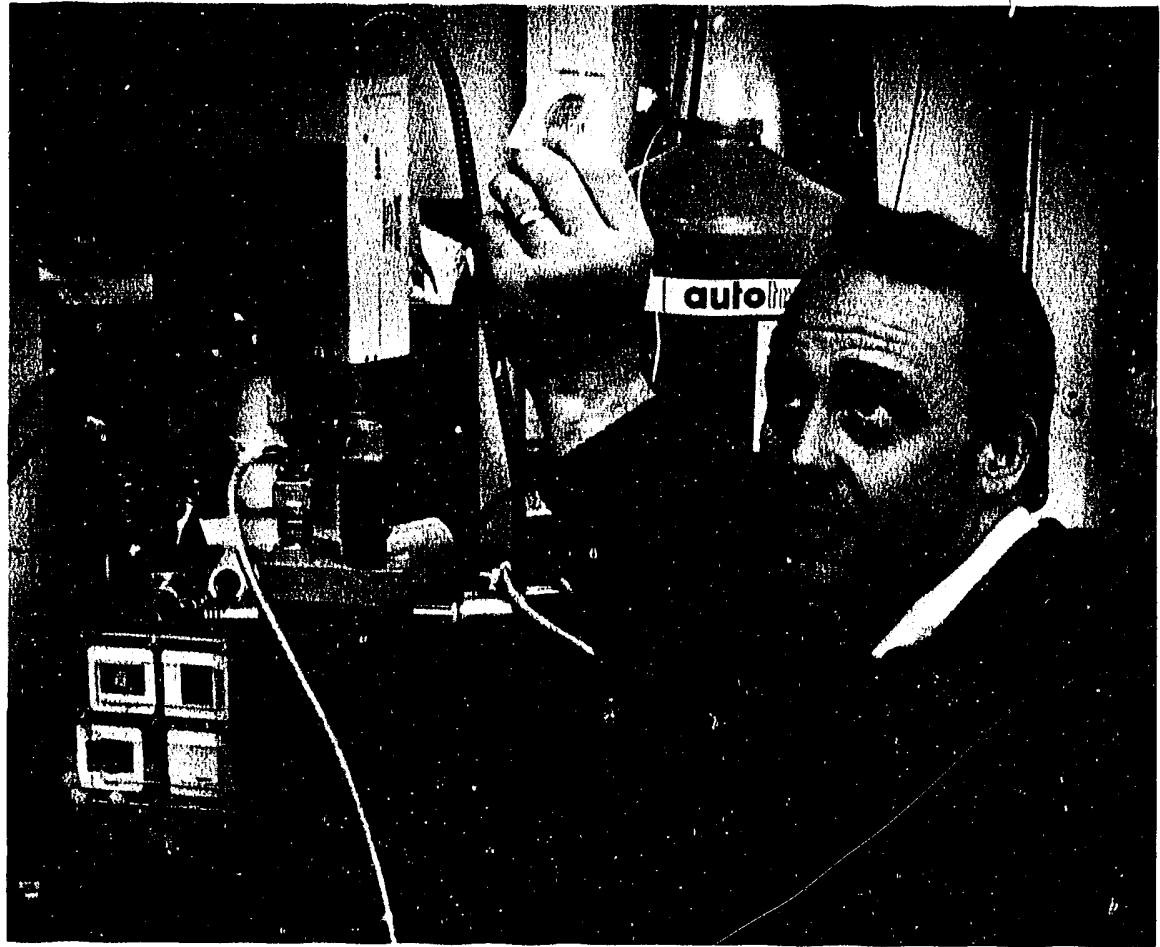

\section{USERS' \\ EXECUTIVE COMMITTEE}

\section{Mark Rivers \\ Univ. of Chicago \\ UEC Chairperson}

The UEC met four times over the year and at each meeting had discussions with both the NSLS and BNL laboratory management on issues of concern to the user community.

The 1991 NSLS Users' Meeting was held on May 20-22. The first day was devoted to five workshops on Atomic and Molecular Science, Computational Tools in XAFS, Electronic and Chemical Phenomena at Surfaces, Imaging, and Surface Structure. The number of workshops has been increas- ing each year, and this year they attracted more than 220 people. The main Users' Meeting was highlighted by an overview of the NSLS by Denis McWhan and ten scientific talks on topics ranging from X-ray Projection Lithography to Mossbauer Spectroscopy. The meeting was attended by over 310 people, which is down just slightly from 1990 . There was a vendor display with 18 vendors and a poster session with over 60 posters of research conducted at the NSLS. Summaries of the main Users' Meeting and the five workshops can be found in Section II of this report.

Both the VUV and $\mathrm{X}$-ray rings have been running well. The X-ray ring has seen significant improvements in beam stability with the addition of both vertical and horizontal feedback systems, improvements which have been high on the users' priority list for some time. Further improvements in 
the VUV feedback system are scheduled for the near future. A. comprehensive program of upgrading the booster ring is under way, which should improve the speed and reliability of injection into both storage rings.

The NSLS has been successful in securing General Plant Projects (GPP) money for two building additions, including an expansion of the experimental floor in the region between $X 1$ and $X 5$. This space will be used in part for new user laboratories. The biology initiative to expand the ring between $\mathrm{X} 5$ and $\mathrm{X} 11$ is still under active consideration by Office of Health and Environmental Research (OHER). These additions should allow every X-ray beamline to have an amount of dedicated setup space.

It appears that the NEXRAD advance weather radar system will definitely be coming to $\mathrm{BNL}$, to be installed on a site about $3 / 4$ mile from the NSLS with operations scheduled to begin in 1994. There is a written agreement that NEXRAD will provide whatever screening is required to reduce $R F$ radiation levels to $2 \mathrm{~V} / \mathrm{m}$ inside the NSLS and other BNL buildings. Additional local shielding will be installed if any experiments are still adversely impacted.

There has been good progress on a number of "quality of life" items affecting users. The recrganization of NSLS space is now almost complete. The new user library/ lounge and the much more convenient location of the $\mathrm{Ij}$ ser Administration Office are among the benefits of these changes for the user community. The Staff Services Division has made a number of improvements in the areas of housing and food services. These include expanded dinner services and the addition of a bar and video games at the Brookhaven Center. A number of apartments are being renovated each

The present members of the UEC are:

\begin{tabular}{|l|l|}
\hline Mark Rivers - Chairperson & Univ. of Chicago \\
\hline Neal Shinn - Vice Chairperson & Sandia National Laboratory \\
\hline Peter Johnson - Past Chairperson & BNL Physics Department \\
\hline Brant Johnson - Secretary & $\begin{array}{l}\text { BNL Applied Science Dept. and } \\
\text { Atomic \& Molecular Science SPIG } \\
\text { Rep. }\end{array}$ \\
\hline C.'T. Chen - Member & AT\&T Bell Laboratories \\
\hline Paul Cowan - Member & Argonne National Laboratory \\
\hline Eric Jensen - Member & Brandeis Univ. \\
\hline John Kirkland - Member & Naval Research Laboratory \\
\hline Gabrielle Long - Member & NIST \\
\hline Larry Sorenson - Member & Univ. of Washington \\
\hline Brian Stephenson - Member & IBM Research Center \\
\hline Susan White-DePace - Ex. Officio & NSLS User Administrator \\
\hline
\end{tabular}

Special Interest Group (SPIG) Representatives

\begin{tabular}{|l|l|}
\hline $\begin{array}{l}\text { Robert Bartynski - UV Photoemis- } \\
\text { sion and Surface Science }\end{array}$ & Rutgers University \\
\hline Charles Bouldin - XAFS & NIST \\
\hline Michael Dudley - Topography & SUNY at Stony Brook \\
\hline $\begin{array}{l}\text { Jonathan Hanson - X-ray Scattering } \\
\text { \& Crystallography }\end{array}$ & BNL Chemistry Dept. \\
\hline $\begin{array}{l}\text { Stephen Lindaas - Students \& } \\
\text { Post-Docs }\end{array}$ & SUNY at Stony Brook \\
\hline \begin{tabular}{l} 
Andrew Sandorfi - Nuclear Physics \\
\hline $\begin{array}{l}\text { Earl Skelton - Energy Dispersive } \\
\text { Diffraction }\end{array}$
\end{tabular} & BNL Physics Dept. \\
\hline $\begin{array}{l}\text { Shawn Williams - Lithography/ } \\
\text { Microscopy/Tomography/X-ray Fluo- } \\
\text { rescence }\end{array}$ & SUNY at Stony Brook \\
\hline
\end{tabular}


The 1992 Scientific Program Advisory Committee (SPSC) chaired by Roger Klaffky and Randy Alkire has been very active. This committee is responsible for identifying significant items which affect the scientific and working environment of the NSLS.

One of the items the UEC has asked this committee to work on is the audio noise level on the experimental floor. The sentiment of the UEC was that users would be willing to abide by noise abatement regulations, such as soundproofing of pumps and equipment racks if it would significantly reduce the noise levels on the floor.

The Space Committee of the UEC is one of the most important com- mittees, recommending policy and implementing space assignments for user labs and offices. This chairmanship of this committee has been handled for many years by the Exxon PRT, first by Rich Hewitt and more recently by Kevin D'Amico. I would like to thank them both for their consid. erable efforts over the years. The new chairman of this committee is Brian Stephenson of IBM.

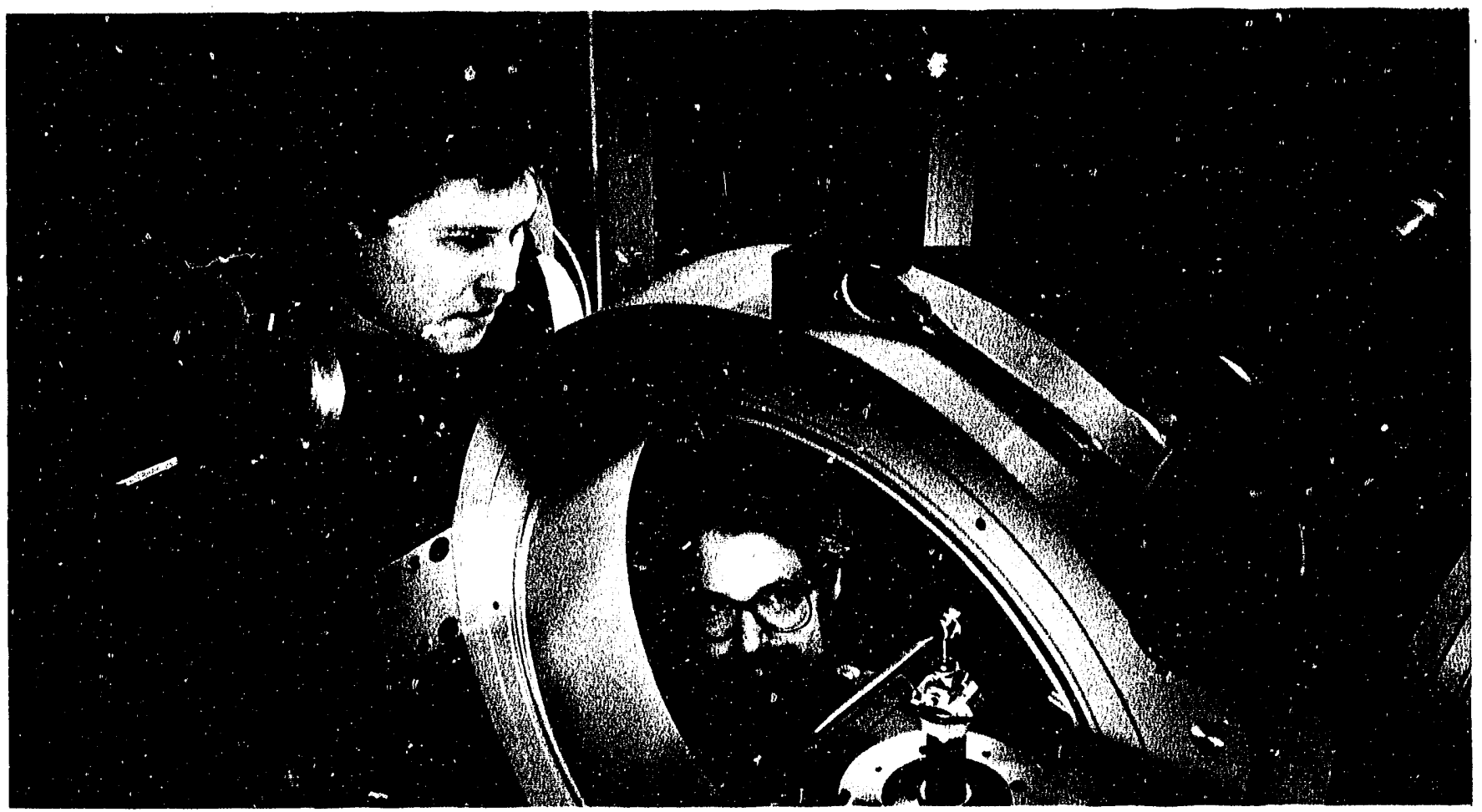

\section{SCIENTIFIC PROGRAM SUPPORT COMMITTEE}

\author{
Randy W. Alkire \\ ANL
}

Roger Klaffky

NSLS

SPSC 91 Co-chairpersons
The 1991 Scientific Program Support Committee was formed in October 1991 and was charged with the task of making recommendations to the UEC that would address user related problems at the NSLS. All aspects of life at the NSLS were considered, including ring operations and quality of life issues. The committee chose to focus on about a dozen topics and final recommendations on these topics will be made in the SPSC 91 report, scheduled to be completed in May 1991. The committee members were:
A. Ackerman - NSLS
R. Alkire - ANL (Co-Chair)
D. Costas - IBM
N. Gmür - NSLS (Secretary)
S. Hulbert - NSLS
B. Johnson - BNL/App. Sci.
J. Kirkland - Sachs Freeman Assoc.

R. Klaffky - NSLS (Co-Chair)

A. MacDowell - AT\&T Bell Labs

K. Pandya - BNL/App.Sci. 


\section{Rothe - NSLS \\ G. Van Derlaske - NSLS \\ S. White-DePace - NSLS}

Ring operations, computing user support facilities, safety and security were the main topics addressed by SPSC 91. In addition, the committee continued to look at several issues examined by previous SPSC committees. Highlights from these investigations will be discussed here, and the complete list of recommendations can be found in the SPSC 91. final report.

Beam stability on both storage rings has been a major topic of concern with the past SPSC committees, and with the installation of global orbit feedback on both rings, beam stability has im. proved dramatically. However, the orbit to which the beam is locked by the global feedback is determined at the beginning of each fill by a global orbit correction algorithm that minimizes the rms sum of the deviations from this orbit of all of the pick-up electrodes (PUEs) around the ring. Since this minimization scheme is global, rather than local, there can be significant fill-to-fill deviations at a given point in the ring. The committee is tracking fill-to-fill changes in beam position at every $\mathrm{X}$-ray Ring dipole magnet in order to determine baseline characteristics for later comparison. A procedure for locally correcting the orbit in every X-ray straight section is being implemented which should reduce fill-to-fill orbit deviations on the adjacent bending magnets.
Xyplex $(9600$ baud terminal server to ethernet) connections are currently available on the VUV Ring and plans for installation on the X-ray Ring are in progress. Some problems with use of the Xyplex system were determined to be caused by too little memory on the Xyplex boards, which have been solved by installing expanded memory boards.

Significant progress has been made by the committee in getting the NSLS trailer park hooked up to Ethernet. All necessary cable has been installed and the final fiber optic link will be completed by early spring 1992 .

The past year has seen a rearrangment of space on the NSLS floor, yielding a more centrally located user administration office, easier access to periodicals, fax and copy machines in the Chasman-Green Memorial Library/Lounge, and more centrally located storage space. Further improvements will be recommended this year regarding the shipping of large items and the user machine shop. Shipping will be made easier and most cost effective by stocking boxes large enough to ship items such as Camac crates, avoiding costly crating charges. The user machine shop steward will begin making recommendations of which raw materials to be put in the stockroom for use in the user machine shop, such as bar stock, Unistrut, and aluminum plate. User training in the machine shop will also be offered to acquaint general users with safe machine shop practices.
Safety is being expanded as a topic by the SPSC and questions regarding scheduling of work by outside contractors, workmen training, and waste disposal are being investigated.

Noise, a topic looked at by all previous SPSC committees, is a problem that will not go away without a significant influx of money. However, the committee has recently purchased sound insulation materials in order to examine the possibility of reducing noise locally by insulating electronics racks and computing equipment.

Theft of equipment at the NSLS has been a major concern of users in the past, primarily during extended shutdown periods. Results of user surveys and an extensive look at police reports show that building 725 does not have a problam with theft, although the same cannot be said for 510. The committee has also looked at personnel security at the NSLS trailer park, and plans are currently underway to install additional lighting around the park to increase illumination in the area.

Other areas in which the users can expect improvement include more frequent filling of vending machines (with a wider variety of food, eg. vegetarian), more routine use of pocket pagers to let users know when ring problems occur, and extended repair services offered through third party ser. vices, accessible to users with an NSLS account. 

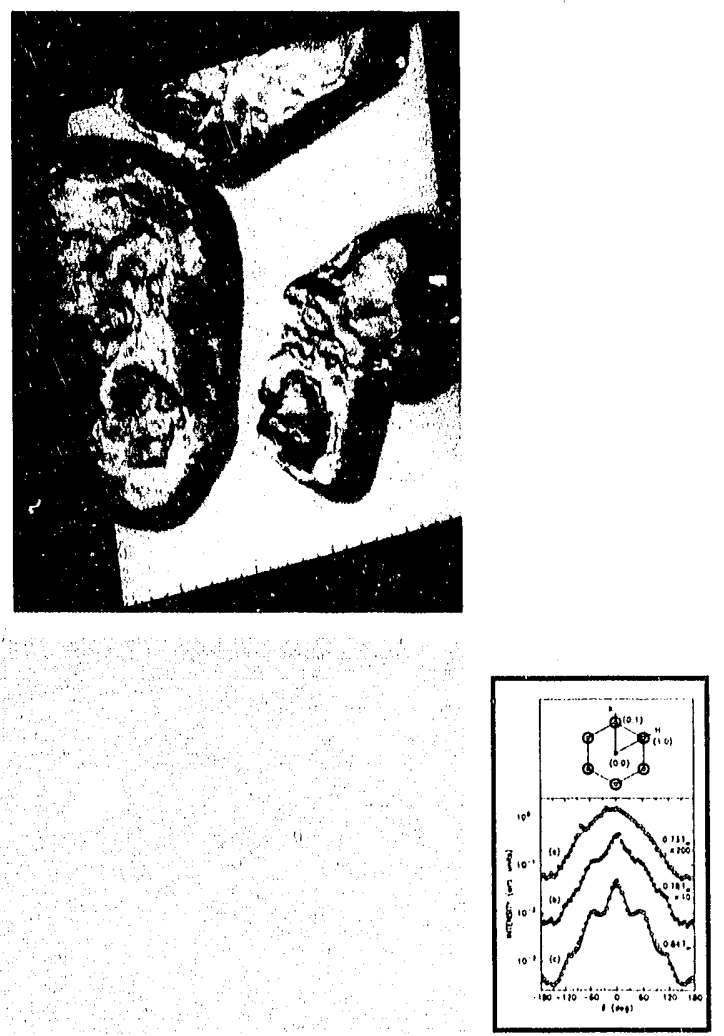

\section{Scientific Disciplines}




\section{ATOMIC AND MOLECULAR SCIENCE}

\section{Mei-Ling Shek \\ NSLS}

\section{Valence electronic excitation}

Beamline U11 is dedicated to gas phase studies involving valence electronic excitations. Of special interest are chemical dynamics in reactions and in van der Waals clusters.

With the use of synchrotron radiation in photoionization mass spectrometry, chemical reaction products are identified, and the photon energy dependence of their yields are measured. "The major channels for the reaction between $\mathrm{N}$ and the $\mathrm{C}_{2} \mathrm{H}_{5}$ radical are sug. gested to be:

$\mathrm{N}+\mathrm{C}_{2} \mathrm{H}_{5}->\mathrm{H}_{2} \mathrm{CN}+\mathrm{CH}_{3}->$ $\mathrm{C}_{2} \mathrm{H}_{4}+\mathrm{NH}$.

A study has been made on the photoionization spectral yields of the radicals $\mathrm{H}_{2} \mathrm{CN}$ and $\mathrm{D}_{2} \mathrm{CN}$, generated by the reactions of $\mathrm{N}$ with $\mathrm{CH}_{3}$ and $\mathrm{CD}_{3}$, respectively.

Recent photoionization and photodissociation experiments on van der Waals clusters include the monomer and clusters of thiophene, $\left(\mathrm{C}_{4} \mathrm{H}_{2} \mathrm{~S}\right)_{\mathrm{n}}$ with $\mathrm{n}=1,2,3$; $\mathrm{CF}_{3} \mathrm{Br}$ and its mixed dimer with
$\mathrm{CH}_{3} \mathrm{OH}$; and complexes of $\mathrm{O}_{2}$ with $\mathrm{C}_{6} \mathrm{H}_{6}$ and $\mathrm{C}_{6} \mathrm{~F}_{6}$. In the last study, a surprising result is that $\left(\mathrm{C}_{6} \mathrm{H}_{6} \bullet \mathrm{O}_{2}\right)^{+}$is formed by the non-dissociative photoionization of the van der Waals dimer $\left(\mathrm{C}_{6} \mathrm{H}_{6}\right) \cdot \mathrm{O}_{2}$ alone. On the other hand, $\mathrm{C}_{6} \mathrm{H}_{6} \mathrm{O}^{+}$and $\mathrm{C}_{6} \mathrm{~F}_{6} \mathrm{O}^{+}$are only produced from larger cluster's by photoionization-induced intracluster reactions. The yield curve for $\mathrm{C}_{6} \mathrm{H}_{6} \mathrm{O}^{+}$in Fig. 1 suggests the mechanism by which the ion is

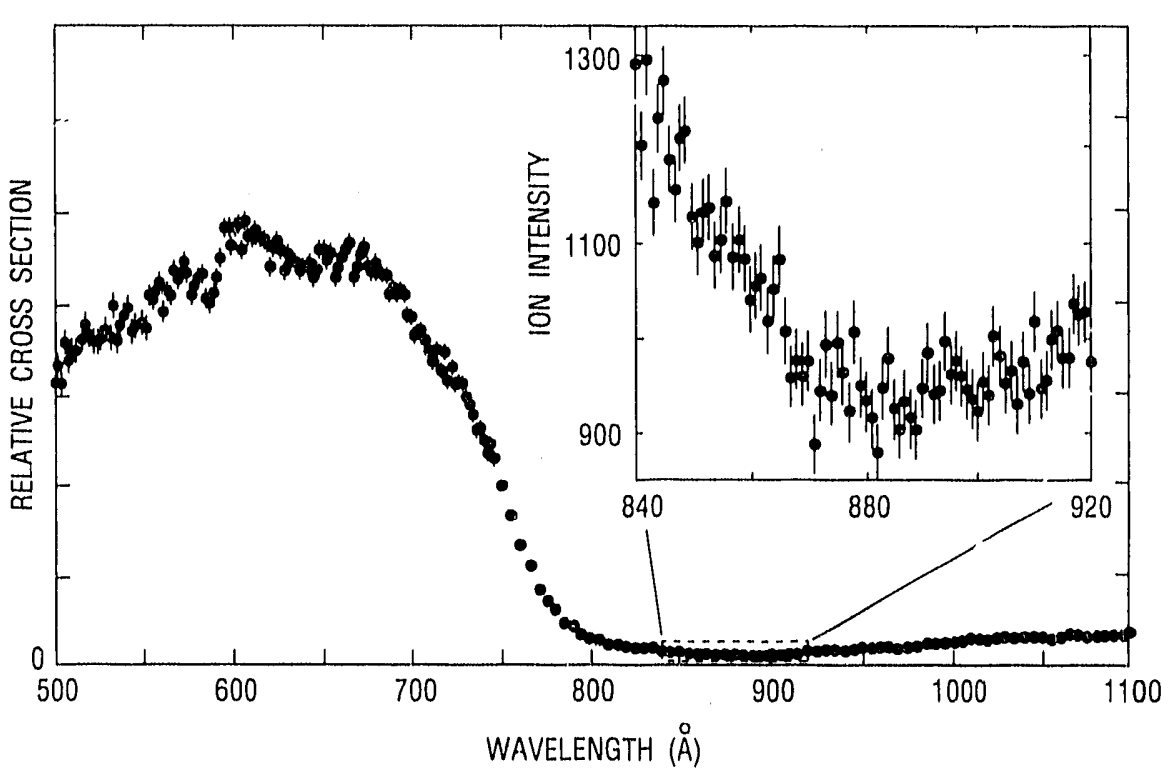

Fig. 1 Yield curve for the production of $\mathrm{C}_{6} \mathrm{H}_{6} \mathrm{O}^{+}$from the van der Waals mixed trimer $\left(\mathrm{C}_{6} \mathrm{H}_{6}\right)_{2} \mathrm{O}_{2}$. The window resonance corresponding to the $\left(\mathrm{c}^{4} \mathrm{\Sigma} \mathrm{u}^{-}\right) 3 \mathrm{~s} \sigma_{\mathrm{g}}$ Rydberg state of free oxygen is prominest at $580 \AA$, showing, for the first time, the involvement of autoionization in intracluster reactions where only polyatomic molecules are present. The inset shows the first onset, at $14.10 \pm 0.05 \mathrm{eV}$, which is far above the thermodynamic threshold. However, it is beautifully consistent with direct onization of a $\mathrm{C}_{6} \mathrm{H}_{6}$ moiety to produce a bound, excited benzene ion corresponding to the ${ }^{2} \mathrm{E}_{1 u}$ or ${ }^{2} \mathrm{~B}_{2 u}$ state, which then transfers its excitation into the neighboring $\mathrm{O}_{2}$, dissociating it with capture of one of the oxygen atoms. Elimination of a $\mathrm{C}_{6} \mathrm{H}_{6}$ moiety disposes enough energy to stabilize $\mathrm{C}_{6} \mathrm{H}_{6} \mathrm{O}^{+}$. J. R. Grover (BNL Chemistry Dept.), G. Hagenow (Freie Univ. Berlin), aid E. A. Walters (U. of New Mexico) 


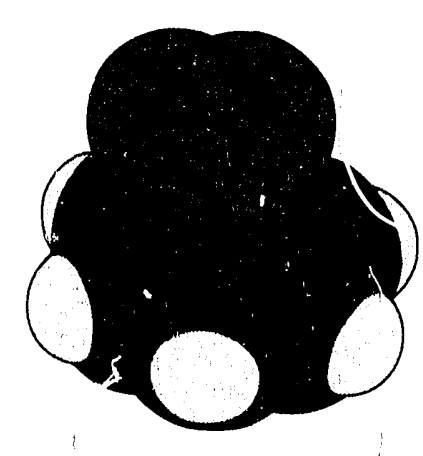

(a)

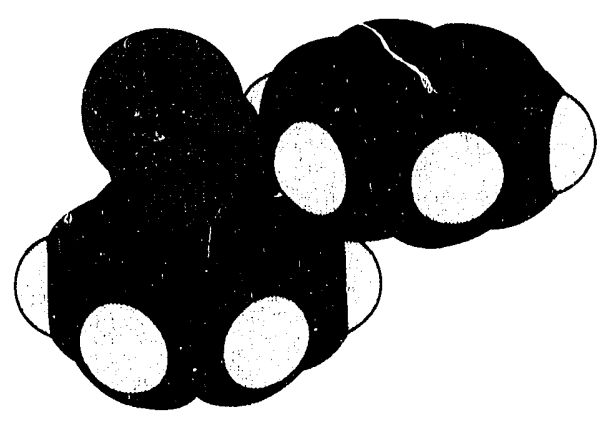

(b)

Fig. 2 Empirically calculated structures of benzene oxygen complexes, assuming that electrostatic and non-bonding forces are dominant. (a) Structure of $\mathrm{C}_{6} \mathrm{H}_{6}{ }^{\circ} \mathrm{O}_{2}$, predicting that the axis of the uxygen molecule is parallel to the plane of th benzene. This is consistent with the failure of this complex to produce $\mathrm{C}_{6} \mathrm{H}_{6} \mathrm{O}^{+}$, because the recoiling $\mathrm{O}$ or $\mathrm{O}^{+}$could never be trapped by the benzene. (b) Structure of $\left(\mathrm{C}_{6} \mathrm{H}_{6}\right)_{2} \mathrm{O}_{2}$, predicting a parallel-displaced structure for the two benzenes, with $\mathrm{O}_{2}$ nestled in the ${ }^{-}{ }^{+} \mathrm{ep}$. Zero-point motion requires that the oxygen and benzenes undergo rotation, so that the $\mathrm{O}_{2}$ axis points at the upper benzene an appreciable fraction of the time. This arrangement is consistent with the observed formation of $\mathrm{C}_{6} \mathrm{H}_{6} \mathrm{O}^{+}$from trimers. J, R. Grover (BNL Chemistry Dept.), G. Hagenow (Freie Univ., Berlin), and E. A. Walters (U. of New Mexico).

\section{Core electronic excitation}

The dynamics of core electron excitatior and de-excitation process in small molecules constitute the main research on Beamline U15. These are probed by ion yield measurements at different angles and electron-ion coincidence measurements. Very recently, the high resolution and high flux on U13UA have enabled refined measurements to be made.

The symmetry and photoabsorption asymmetry parameter $\beta$ of core-hole excited molecular states are investigated by looking at the angular dependence of photo-fragmentation. Ion yield spectra are recorded simultaneously by two retarding field analyzers with their axes oriented perpendicular and parallel to the electric vector of synchrotron radiation. The excitation of a series of diatomic molecules $\left(\mathrm{N}_{2}, \mathrm{O}_{2}, \mathrm{NO}\right.$ and $\mathrm{CO}$ ) near the $\mathrm{N}$ and $\mathrm{O} \mathrm{K}$-edges shows the general pattern of a preedge $\pi^{*}$ resonance and a $\sigma^{*}$ resonance. In addition, the $\beta$-spectra of NO at ihe N K-edge and the 0 $\mathrm{K}$-edge are found to be qualitatively similar to the corresponding $\beta$-spectra of $\mathrm{N}_{2}$ and $\mathrm{O}_{2}$, respectively. A second study is directed towards triatomic molecules with different symmetries, namely, $\mathrm{CO}_{2}\left(\mathrm{D}_{\infty \mathrm{h}}\right), \mathrm{N}_{2} \mathrm{O}\left(\mathrm{C}_{\infty \mathrm{v}}\right)$ and $\mathrm{H}_{2} \mathrm{O}$ $\left(\mathrm{C}_{2 \mathrm{v}}\right)$; the ion yields and the anisotropy parameters at the $\mathrm{OK}$ edges as well as other atomic edges have been determined.

The chemistry induced by core electron excitation is studied by electron multiple ion coincidence measurements. The behavior of $\mathrm{CO}$ upon $\mathrm{O} 1 \mathrm{~s}$ photoionization is studied by mass spectrometry in coincidence with $465.475 \mathrm{eV} \mathrm{Au}$ - ger electrons, which produce two $\mathrm{CO}^{+}$siates with known energies. Their fragmentation to $\mathrm{C}^{+}$and $\mathrm{O}^{+}$ leaves these atomic ions in highly excited electronic states, as deduced by the difference in the parent ion energies and the kinetic energies of the atomic ions. The distribution of energies in other fragmentation pathways giving dipositive ions is also obtained. In a study of $\mathrm{NH}_{3}$ upon the creation of a N1s core hole, the mass spectrum in coincidence with $369-379$ $\mathrm{eV}$ Auger electrons gives the dissociation dynamics of $\mathrm{NH}_{3}{ }^{2+}$ in its ground singlet and first excited electronic states. A recent development is the detecticn of ion pairs, and a study is made on the fragmentation of methanol following thie excitation of $\mathrm{C} 1 \mathrm{~s}$ to the continuum (see Fig. 3). 


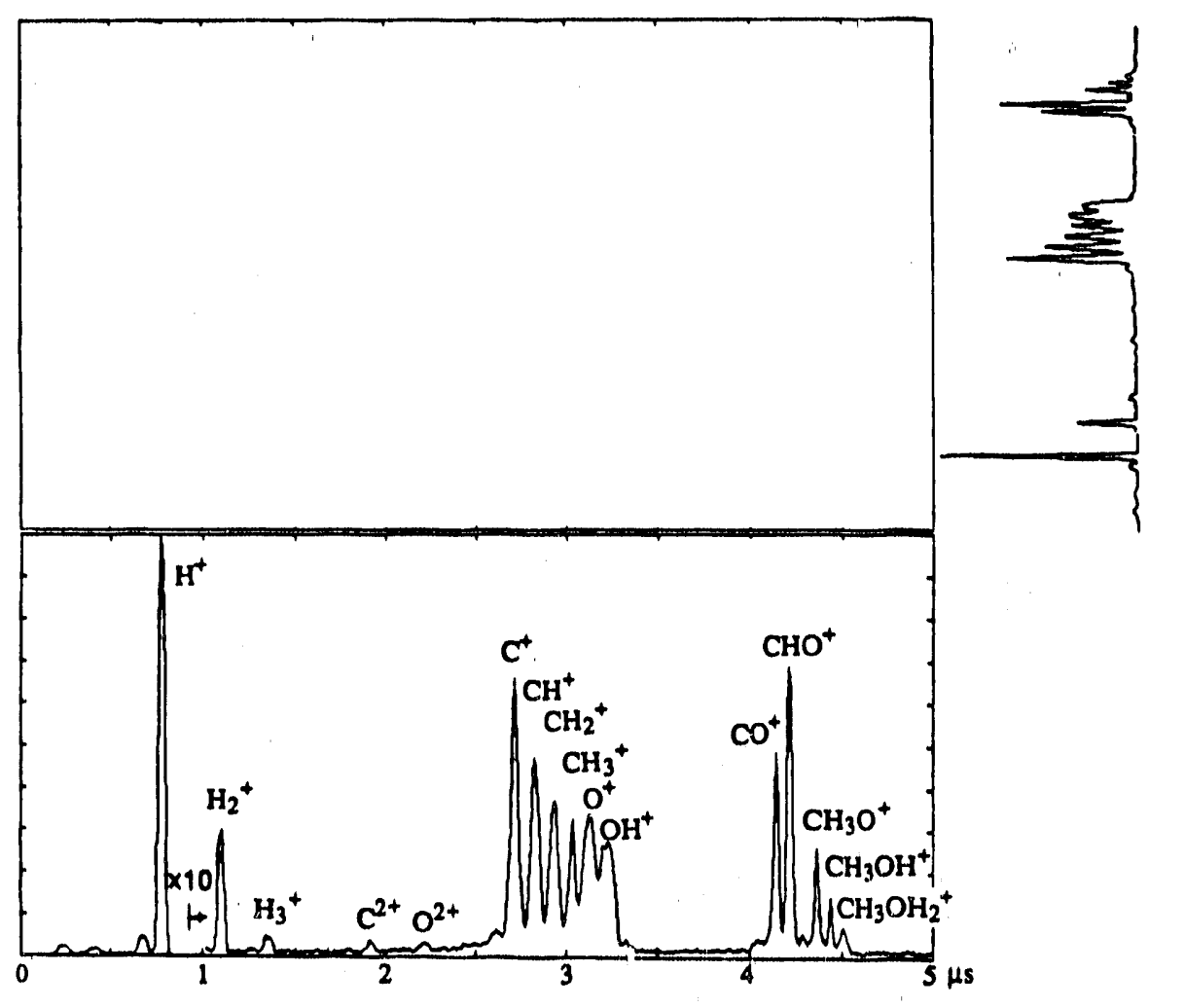

Fig. 3 The dynamics of molecular fragmentation following core excitation probed by electron-multiple ion coincidence spectroscopy. The sequential nature of the fragmentation process is revealed here by a two-dimension ion-ion coincidence map of methanol $\left(\mathrm{CH}_{3} \mathrm{OH}\right)$ after ionization of the carbon $1 \mathrm{~s}$ electron. The dense lobes in the map for the coincidence between $\mathrm{O}^{+}$and $\left[\mathrm{C}^{+}, \mathrm{CH}_{\mathrm{n}}{ }^{+}(\mathrm{n}=1,2,3)\right]$ exhihit a slope of -1 as a result of axial recoil in a two body fragmentation process. This result indicates that the neutral particles are ejected earlier in time. C.I, Ma, K. Lee, D. Y. Kim, and D, M. Hanson (SUNY at Stony Brook).

Chemically important pathways of core hole decay of large molecules may often include fragmentation involving neutral species in an excited state. A complete characterization of the dynamics of core hole decay of such molecules would not be possible without information about the neutral decay products. An ideal technique for observation of the neutral frag. ments is the observation of luminescence from excited states of such fragments (see Fig. 4). From a knowledge of the excitation energy of the atomic species and the final state energies of the Auger decay products it is possible to determine the probable final states that result in the observed fragments.

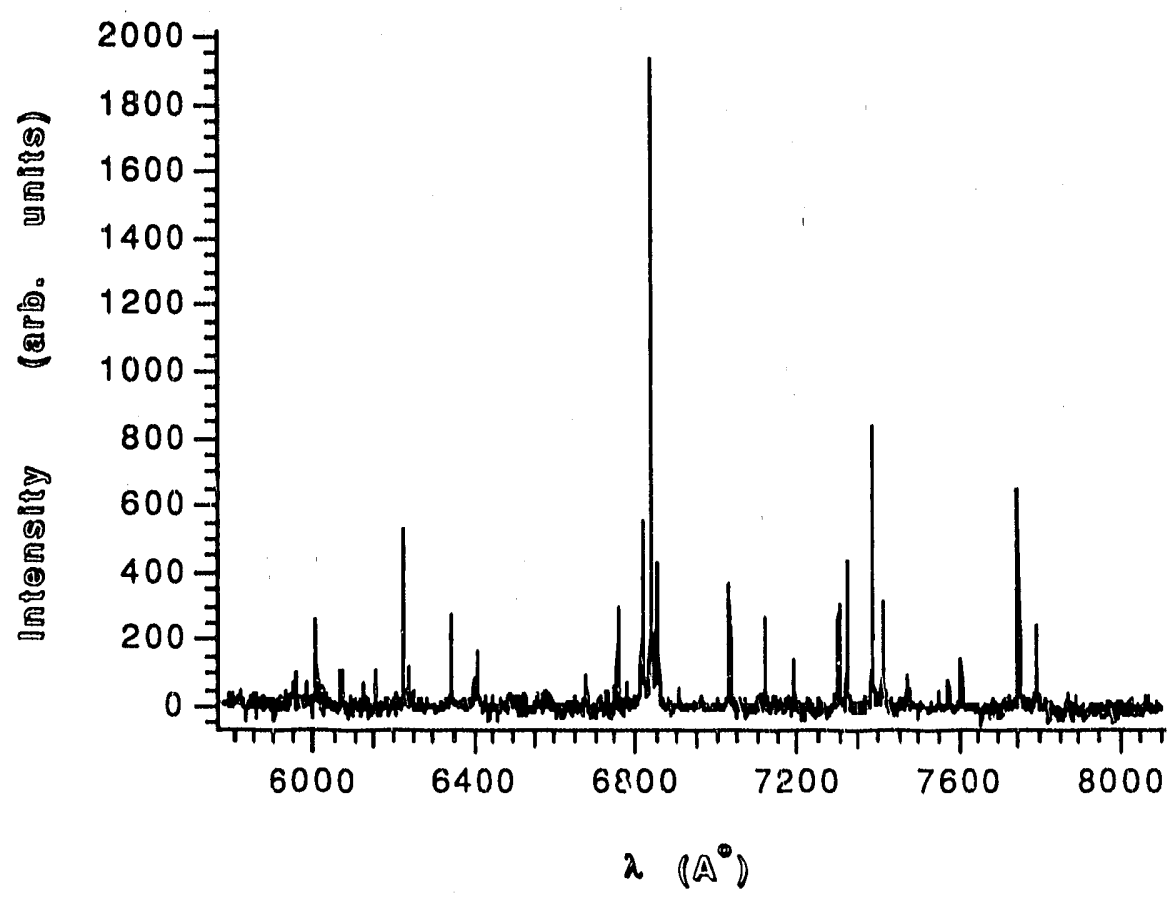

Fig. 4 Intensity of neutral atomic fluorine luminescence from $\mathrm{CF}_{4}$ following $\mathrm{Cls}$ core excitation. M. Mahalingham, C. Woodbrige, K. Lee, and D. M. Hanson (SUNY at Stony Brook). 
On Beamline U13UA, two recent gas phase experiments have been done. For $\mathrm{N}$ 1s photoabsorption in $\mathrm{N}_{2}$, angular-dependent ion yield measurements have identified excitations to $\pi$ an $\sigma$ symmetry states and refined a previous peak assignment obtained by high-resolution absorption spectroscopy on U4B (see Fig. 5).

The second experiment demonstrates the effect of site and state specificity in molecular core hole decay. The $\mathrm{CK}$-edge resonances in acetone in Fig. 7, obtained by total ion yield, show $1 \mathrm{~s} \rightarrow \pi^{*}$ and $1 \mathrm{~s}$ $\rightarrow 3 \mathrm{p}$ excitations from different carbon atoms. The identification is made possible from the Auger electron spectra at resonance together with electron-ion coincidence data (see Fig. 6).

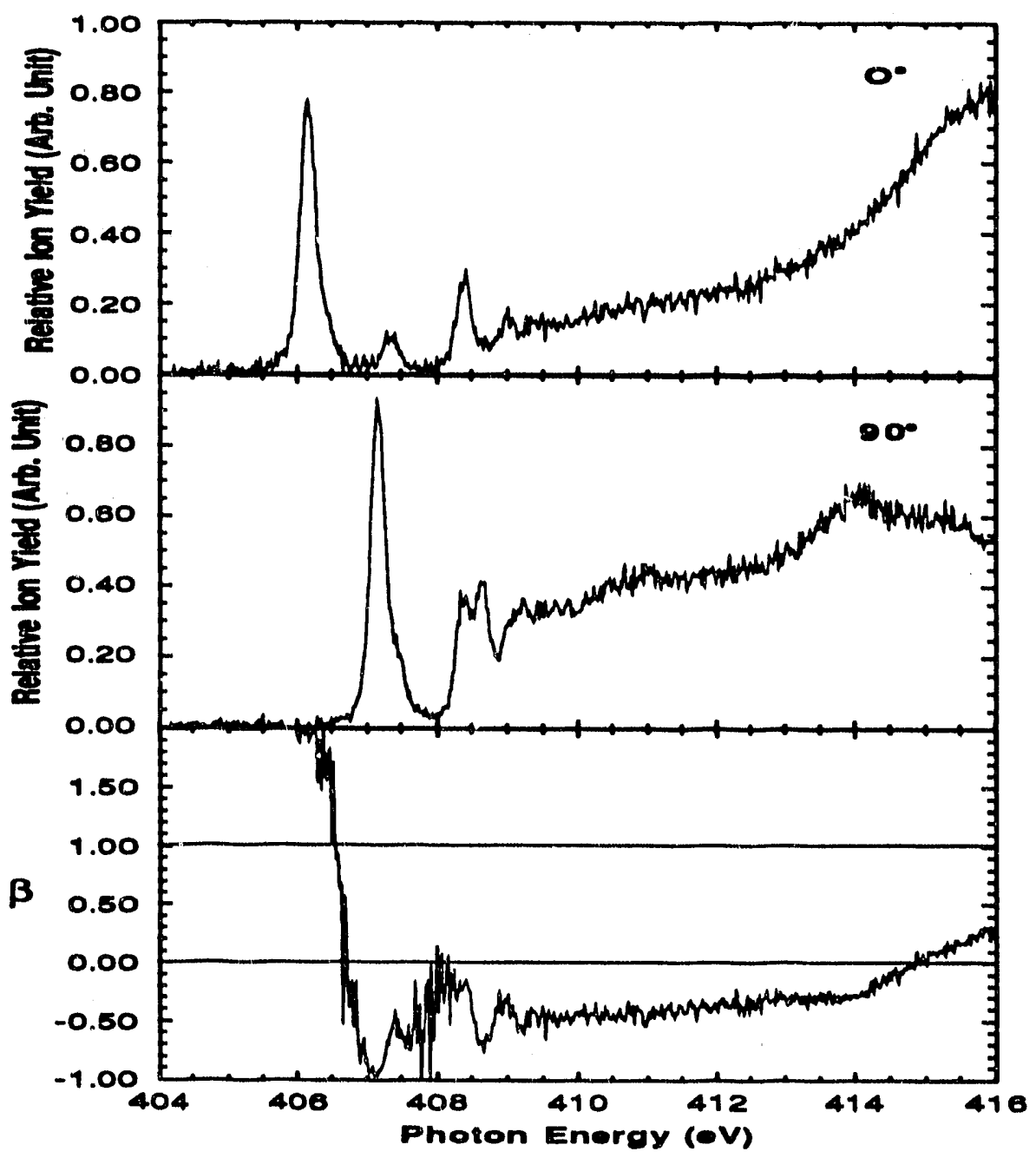

Fig. 5 Ion Yields from $\mathrm{N}_{2}$ at $0^{\circ}$ and $90^{\circ}$, and the anisotropy parameter, $\beta$, spectra. The $\beta$ spectrum is constructed from the ion yields at the two detectors and the degree of light polarization. Excitations to symmetry electronic states appear only at $90^{\circ}$, and excitation to $\Sigma$ symmetry electronic states appear only at $0^{\circ}$. Resonance peaks that have not been resolved by conventional high resolution spectroscopy can now be revealed in the two different detectors. D. Y. Kim, K. Lee, C.-I. Ma, M. Mahalingham, and D. M. Hanson (SUNY at Stony Brook), and S. L. Hulbert (NSLS). 


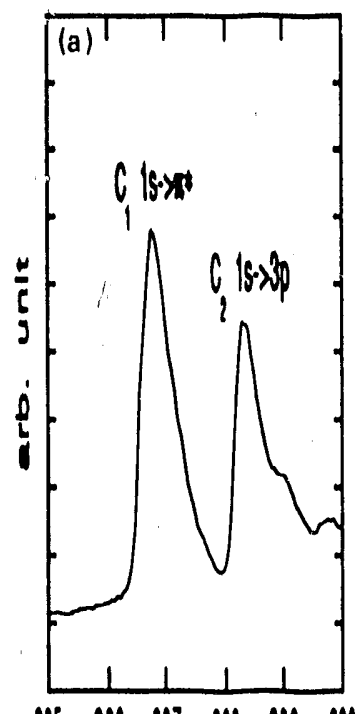

$265 \quad 266 \quad 287 \quad 280269298$

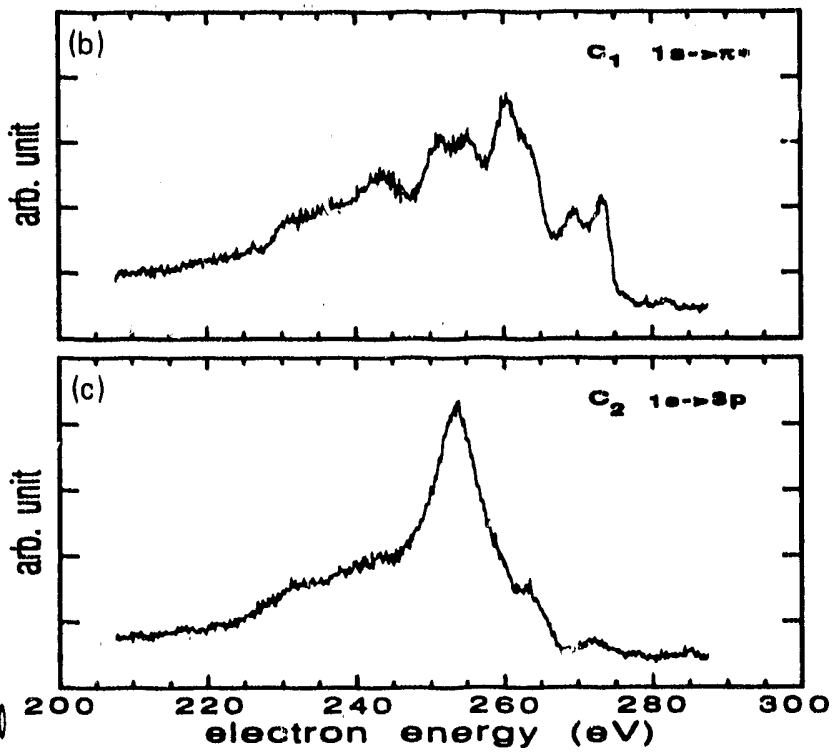

$\operatorname{hr}(\mathrm{el})$
Fig. 6 (a) C K-edge resonances in acetone, showing the $1 \mathrm{~s} \rightarrow \pi^{*}$ resonance of the carbonyl carbon $\left(\mathrm{C}_{1}\right)$ and the $1 \mathrm{~s} \rightarrow 3 \mathrm{p}$ resonance of the methyl carbon $\left(C_{2}\right)$. The assignment is made from the Auger electron spectra at resonance $(b, c)$ together with electron-ion coincidence data. For example, the spectral shape of (c) resembles the Auger spectrum of methane, which implies that $\mathrm{C}_{2}$ is the methyl carbon atom and spectator decay dominates the core hole relaxation. By elimination, $\mathrm{C}_{1}$ is the carbonyl carbon atom. C.-I. Ma, K. Lee, D. Y. Kim, and D. M. Hanson (SUNY at Stony Brook) and S. L. Hulbert (NSLS).
III. High resolution core level excitation and de-excitation On the newly completed X1B beamline, core level spectroscopies in small molecules have been done. The combination of undulator radiation and high-resolution soft x-ray optics has resolved features in the $\mathrm{O} \mathrm{K}$-edge photoabsorption spectra of $\mathrm{O}_{2}$ more clearly than before. The capability for core level photoelectron spectroscopy at vibrational resolution is also demonstrated, as shown in Fig. 7. The vibrational fine structure in the decay of core hole states in gas phase $\mathrm{N}_{2}$ reveals information about the coupling between electronic states and nuclear motion, both evolving on a time scale of $\sim 10^{-14} \mathrm{~s}$. The first observation of vibrational selection on core hole decay is shown in Fig. 8. The high intensity has also enabled the observation of electronic decay in the $1 \mathrm{~s}^{1} 3 \mathrm{p}$ and $1 \mathrm{~s}^{-1}$ 3s core hole Rydberg states, together with the much more intense $1 \mathrm{~s}^{-1}$ and $1 \mathrm{~s}^{-1} \pi^{*}$ decays. A com. parison yields information on the spectator Rydberg electron and Coulomb interactions between the the rest of the system.

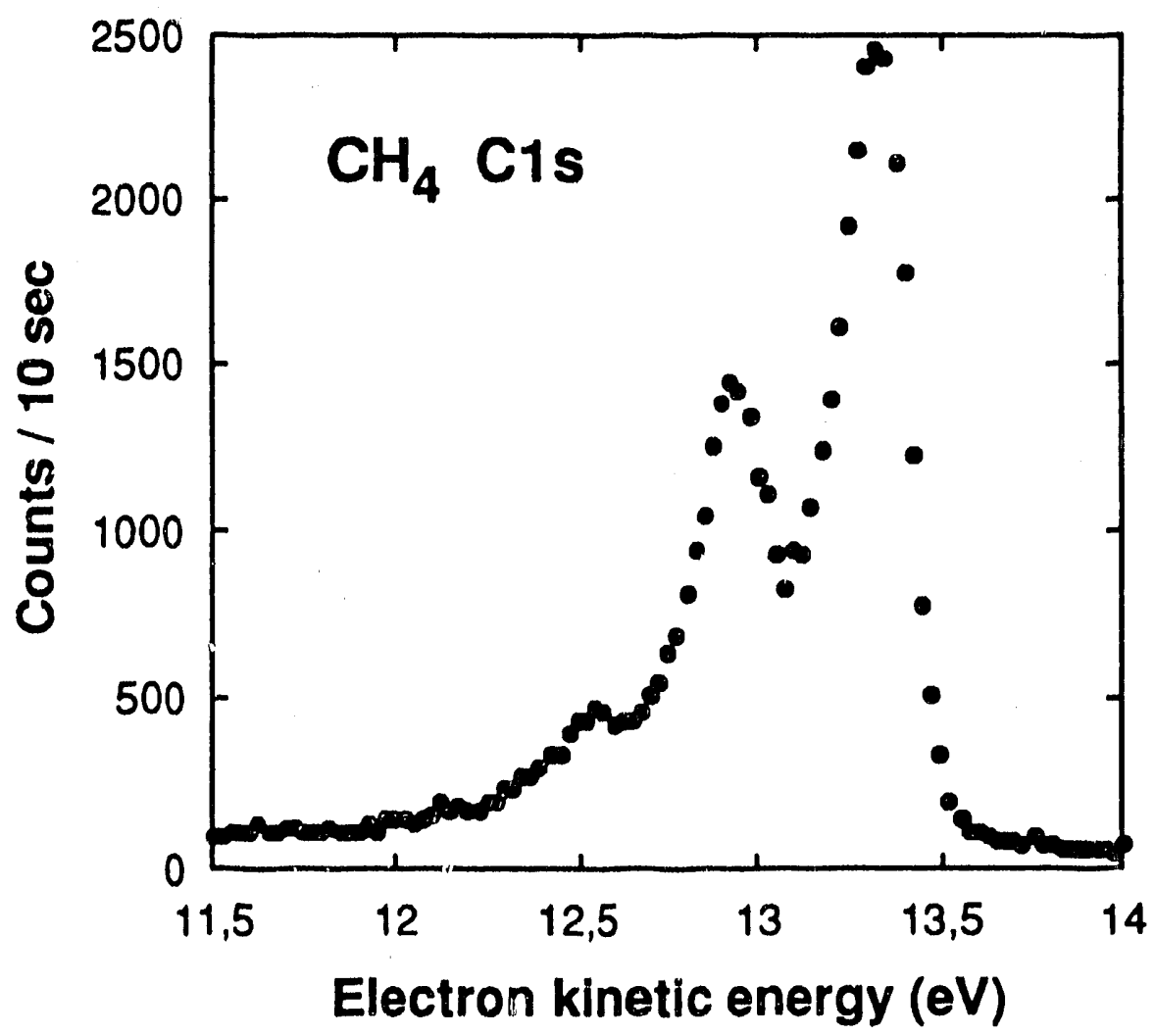

Fig. $7 \mathrm{C} 1 \mathrm{~s}$ photoelectron spectrum of methane taken at a photon energy of $\sim 304 \mathrm{eV}$ on X1R, clearly resolving the vibrational peaks. K. J. Randall, J. Feldhaus, A.L.D. Kilcoyne, A. M. Bradshaw (Fritz-Haber-Institut.), W. Eberhardt, J. E. Rubensson (KFA Jülich), Y. Ma (PNL), Z. Xu, and P. D. Johnson (BNL Physics Dept.). 


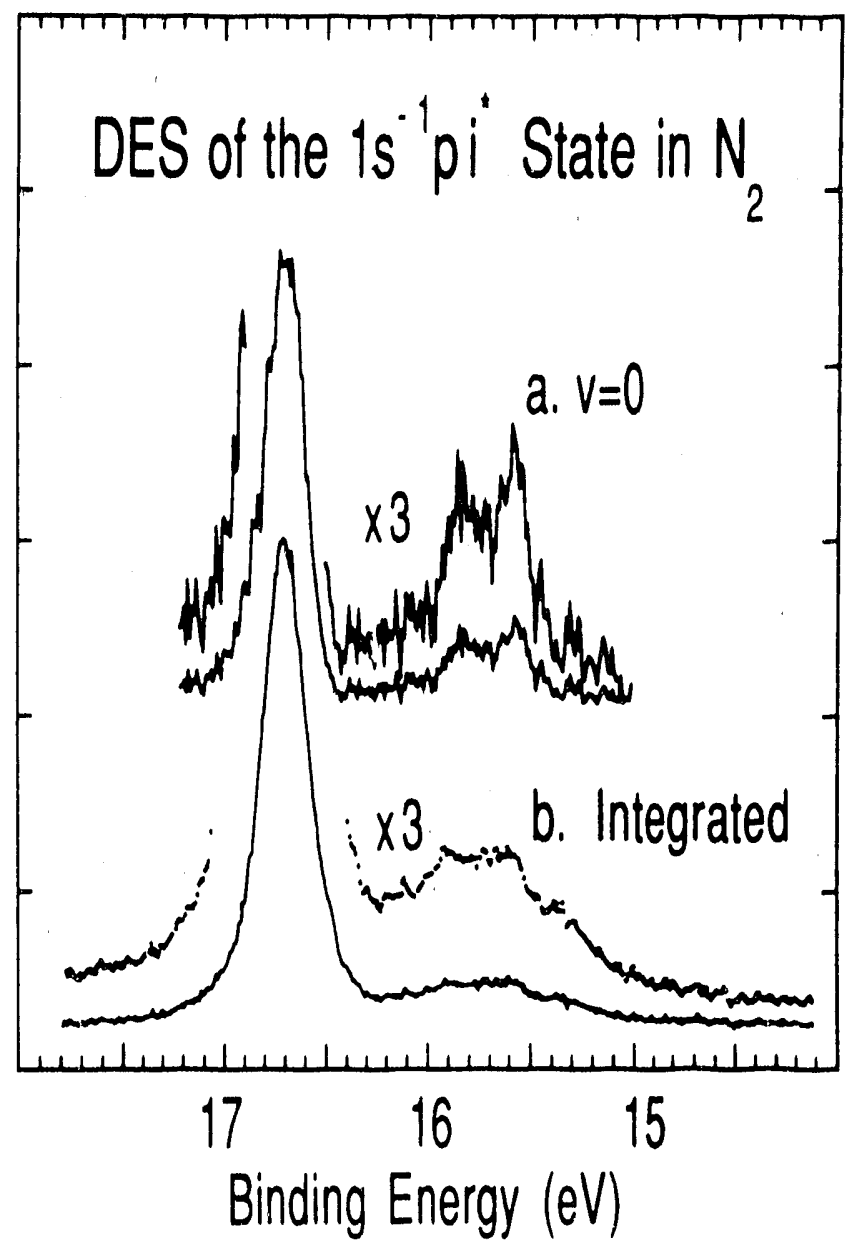

Fig. 8 (a) The decay of the first vibronic level of the $1 \mathrm{~s}^{-1} \pi^{*}$ state of gas phase $\mathrm{N}_{2}$, obtained on beamline $\mathrm{X} 1 \mathrm{~B}$. The sharp peak at $16.7 \mathrm{eV}$ corresponds to the first vibrational level of the final state with a $1 \pi_{\mathrm{u}}$. hole, and the weaker progression starting at $15.6 \mathrm{eV}$ corresponds to the first vibrations of the final state with a $3 \sigma_{\mathrm{g}}$ hole. (b) Previously recorded spectrum without vibrational resolution in the excitation. J. E. Rubensson, W. Eberhardt (KFA Jülich), Y. Ma (PNL), Z. Xu, and P. D. Johnson (BNL Physics Dept.).
IV. X-ray Photoionization

On beamline X24A, the double photoionization of $\mathrm{He}$ at $2.8 \mathrm{KeV}$ has been studied. The ratio $\mathrm{He}^{++} /$ $\mathrm{He}^{+}$is found to be $1.6 \pm 0.3 \%$, presenting the first accurate measurement at photon energies greater than $200 \mathrm{eV}$. This measurement is important because the photoionization of $\mathrm{He}$ is the testing ground for understanding electron correlation.

On beamline X26C, initial studies have been made of the double differential cross-sections of Compton-ejected electrons from $\mathrm{Ne}$, using 10-15 keV photons obtained by filtering white light with a thick Be window and $3 \mathrm{~mm}$ Kapton. A spherical sector electrostatic spectrometer has been used to detect electron kinetic energies up to $4.5 \mathrm{keV}$. Modifications to the gas jet, the scattering chamber, and plans for a magnetic spectrometer to detect up to 100 $\mathrm{keV}$ electrons, are under way. 


\section{ENERGY}

DISPERSIVE

DIFFRACTION

\section{Earl Skelton \\ NRL}

Special Interest Group

Representative

Energy dispersive diffraction techniques at NSLS are principally used for high pressure research. Extreme pressures, in excess of those found at the center of the earth $(\approx 360 \mathrm{GPa})$, are developed in the submillimeter regions between the flattened tips of opposed diamond-anvils. Larger volume high pressure capabilities are available in the cubic press, operational on X17B. During the past year, some diamondanvil cell energy dispersive diffraction studies were pursued on $\mathrm{X} 7 \mathrm{~A}$, but the bulk of the work was performed on the beamline designed for this technique, $\mathrm{X} 17 \mathrm{C}$.

Using $\mathrm{X} 7 \mathrm{~A}$ in the white beam mode, Wang et al. from the University of Hawaii studied the isothermal compression of spinel $\left(\mathrm{Fe}_{2} \mathrm{SiO}_{2}\right)$ at pressures up to 24 GPa. They observed an apparent stiffening of the spinel lattice above $18 \mathrm{GPa}$, which they attributed to nunhydrostatic conditions. In a separate study, Reichlin et al. from Lawrence Livermore $\mathrm{Na}$ tional Laburatory (LLNL), in collaboration with Hemley et al. from Carnegie Institution of Washington (CIW), found that monatomic metallic iodine trans- forms to a bcc structure at 55 $\mathrm{GPa}$, which prevails to at least 181 $\mathrm{GPa}$. At this pressure, the volume compression, $\mathrm{V} / \mathrm{V}_{0}$, is measured to be 0.356 .

Zhao et al. from SUNY at Stony Brook, using the cubic press on $\mathrm{X} 17 \mathrm{~B}$, measured both the thermal expansivity and the isothermal compressibility of $\mathrm{NaMgF}_{3}$. At ambient pressure and a temperature of $765^{\circ} \mathrm{C}$, a phase transformation was observed from the orthorhombically distorted perovskite structure (Pbnm) to a cubic phase $(\mathrm{Pm} 3 \mathrm{~m})$. Results from these measurements allow deductions about the iron content of the earth's core.

A broad spectrum of energy dispersive diffraction work has been performed on $\mathrm{X} 17 \mathrm{C}$, ranging from structure determinations of crystals with volumes as small as 28 attoliters to studies at pressures approaching $300 \mathrm{GPa}$ and at temperatures ranging from 50 to $900 \mathrm{~K}$.

Loubeyre et al. from Universite Paris, collaborating with scientists from CIW, measured the phase diagram and equation of state of helium over the range from 50 to $300 \mathrm{~K}$ and from 4 to 20 $\mathrm{GPa}$. Relations between the fluid phase and the two condensed phases, fcc and hcp, were determined from energy dispersive measurements on polycrystalline samples (see Fig. 9).

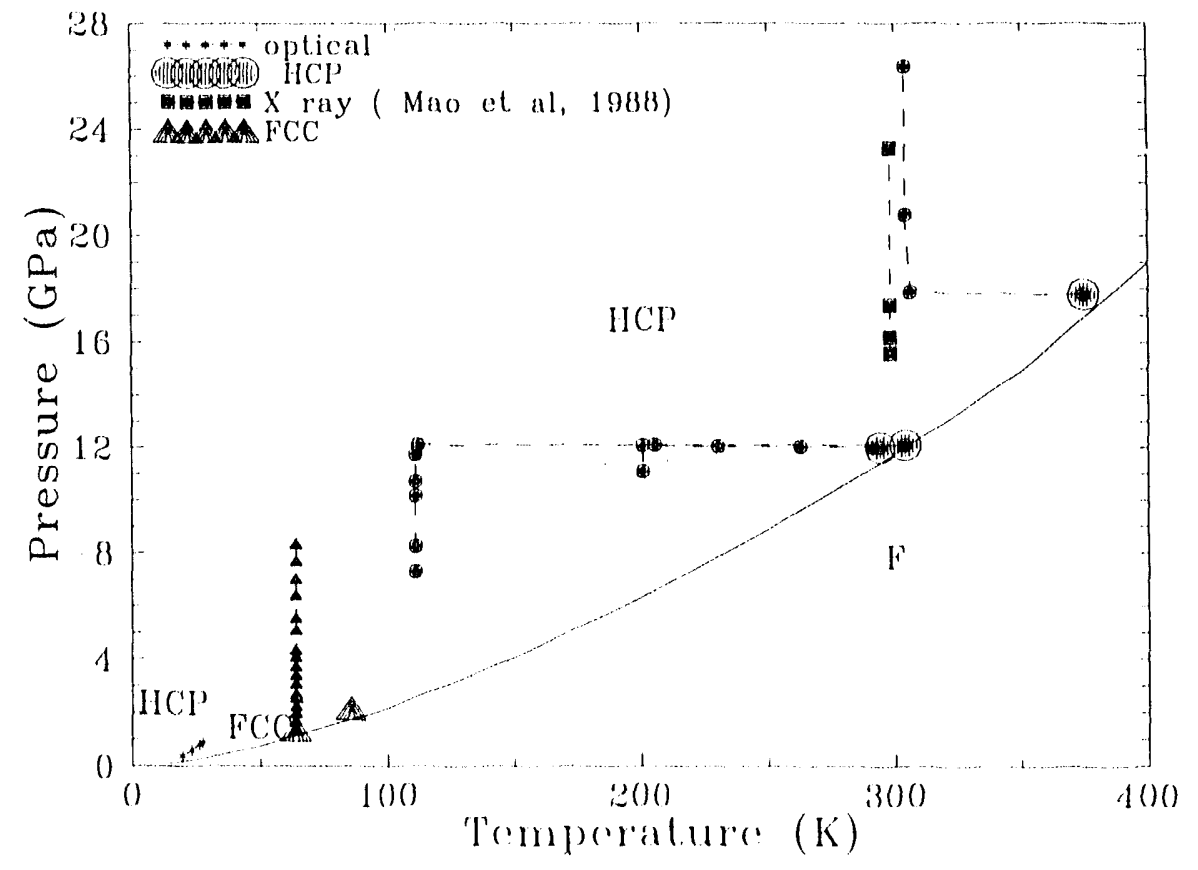

Fig. 9. Phase diagram of ${ }^{4} \mathrm{He}$ measured at high pressure using X17C. The large symbols in. dicate where single crystals had been grown from the melt by the isochoric method. $P$. Loubeyre et al. (Universite Paris). 
In a similar study, but on a single crystal sample, Mao et al. from CIW confirmed that hydrogen crystallizes in the hcp structure up to at least $42 \mathrm{GPa}$. Data from this sample allowed fitting the BirchMurnaghan equation of state to over $40 \mathrm{GPa}$ (see Fig. 10).

A number of high pressure in. vestigations were pursued on a

Fig. 10. Pressure-volume relation of solid hydrogen at room temperature, measured at $X 17 \mathrm{C}$ up to a pressure of $42 \mathrm{GPa}$. Fitting the data to the Birch-Murnaghan equation of state yields an isothermal bulk modulus $\mathrm{K}_{0}=0.492 \mathrm{GPa}$ and $\mathrm{K}={ }_{0}^{\prime} 4.3$. H. K. Mao et al. (Carnegie Institution of Washington). variety of materials. From CIW, magnesiowsitite, $\left(\mathrm{Mg}_{0.6} \mathrm{Fe}_{0.4}\right) \mathrm{O}$ was examined to $30 \mathrm{GPa}$ and 800 $\mathrm{K}$ by Fei et al.; Hu et al. collected data on possible high pressure calibrants, $\mathrm{W}$ and $\mathrm{Pt}$, to hun dreds of GPa (see Fig. 11); Vos et al. performed measurements to 10 $\mathrm{GPa}$ and elevated temperatures to chart the phase diagrams of $\mathrm{N}_{2}$, $\mathrm{He}$, and $\mathrm{N}_{2} \cdot \mathrm{He}$ mixture (see Fig. 12); and Meade et al. performed studies on silica glass to $42 \mathrm{GPa}$, the results of which can be interpreted as an increase in the short and intormediate range order with increasing pressure (see Fig. 13).
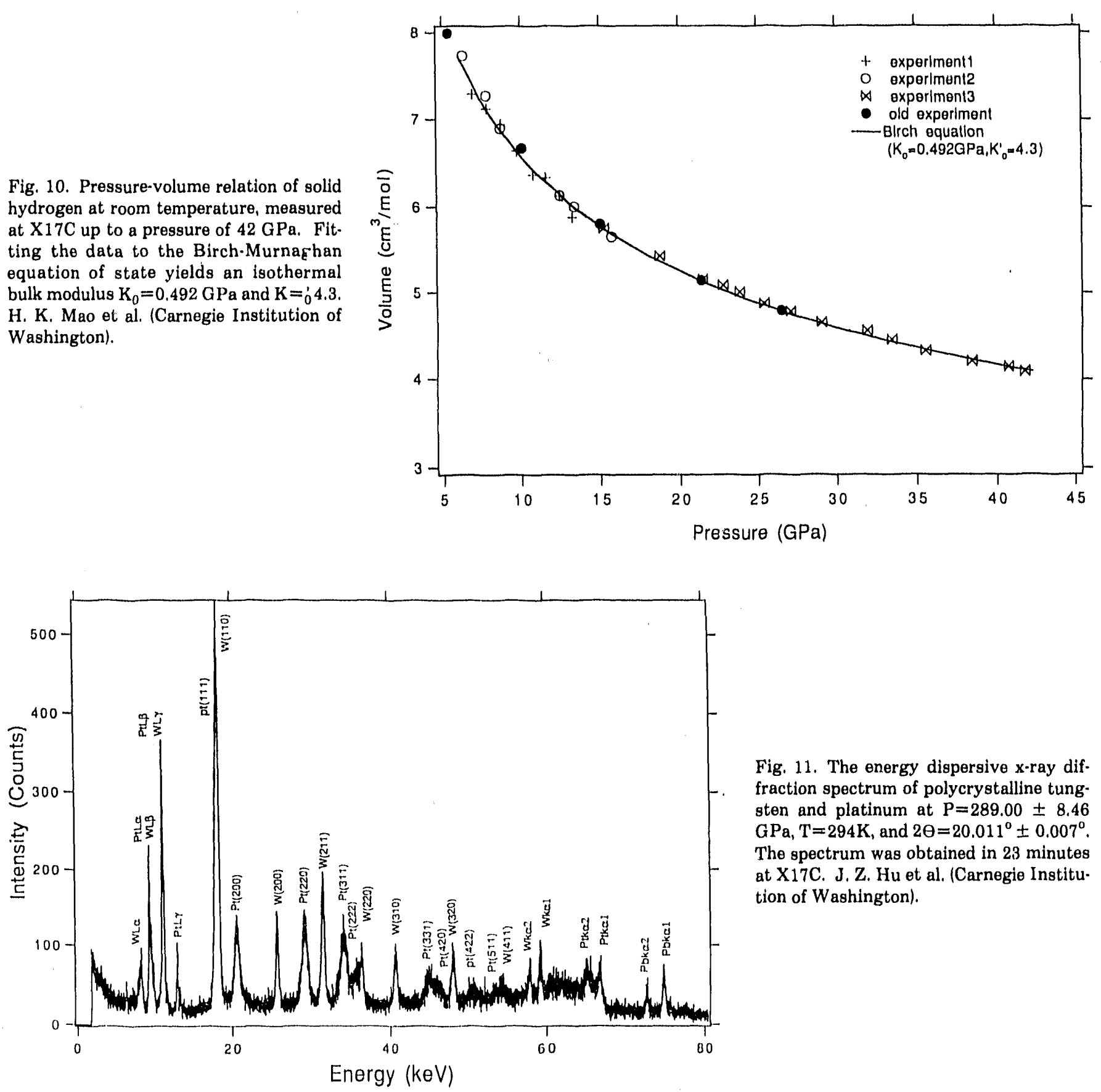

Fig. 11. The energy dispersive x-ray diffraction spectrum of polycrystalline tungsten and platinum at $\mathrm{P}=289.00 \pm 8.46$ $\mathrm{GPa}, \mathrm{T}=294 \mathrm{~K}$, and $2 \theta=20.011^{\circ} \pm 0.007^{\circ}$. The spectrum was obtained in 23 minutes at X17C. J. Z. Hu et al. (Carnegie Institution of Washington). 


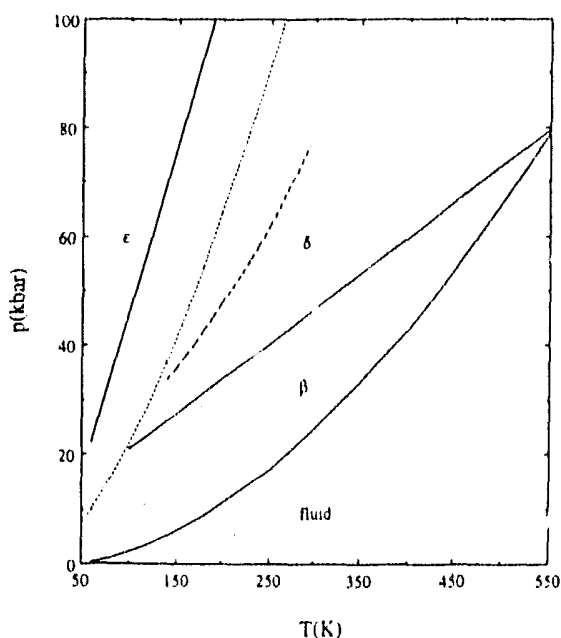

Fig. 12. P.T phase diagram of $\mathrm{N}_{2}, \mathrm{He}$, and $\mathrm{N}_{2} \cdot \mathrm{He}$ mixture measured at X17C. The solid curves are, in order of increasing pres. sure, the $\beta$-fluid, $\delta-\beta$, and $\epsilon \cdot \delta$ phase boundaries of pure $\mathrm{N}_{2}$; the short dashed curve is the melting curve of helium; and the dashed curve is the $\epsilon^{*}-\delta^{*}$-fluid three phase boundary of the $\mathrm{N}_{2} \cdot$ He mixture. W. L. Vos et al. (Carnegie Institution of Washington).

Qadri et al. from NRL examined phase transitions in $\mathrm{Zn}_{1-x} \mathrm{Fe}_{x} \mathrm{Se}$ and $\mathrm{Hg}_{1-\mathrm{x}} \mathrm{Fe}_{\mathrm{x}} \mathrm{Se}$ (see Fig. 14).

Akella et al. from LLNL studied the equation of state of $\mathrm{Nd}$ to 90 $\mathrm{GPa}$.

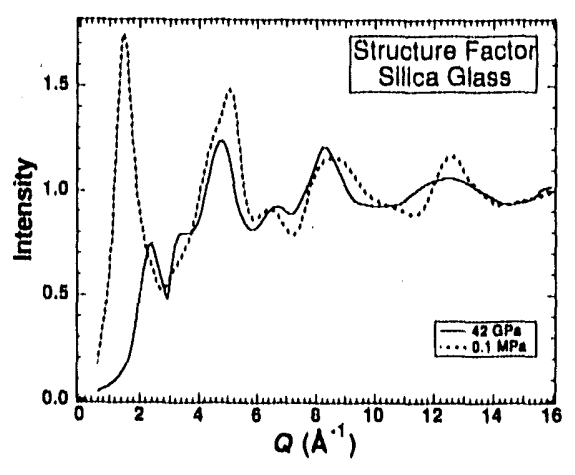

Fig. 13. The structure of $\mathrm{SiO}_{2}$ glass at high pressure. To enable a new generation of structural studies on liquids and glasses, $X 17 C$ researchers from the Geophysical Laboratory of the Carnegie Institution of Washincton have developed $x$-ray diffraction techniques for amorphous materials using the diamond anvil cell at high pressures. This work will have direct applica. tions to the study of silicate melts deep within the earth and to theoretical investigations of liquid and glass structure. They have measured $x$-ray diffraction spectra of approximately a $4 \times 10^{-13} \mathrm{~m}^{3}$ volume of $\mathrm{SiO}_{2}$ glass in the diamond anvil cell at 42 $\mathrm{GPa}$ and room temperature. This figure compares the $\mathrm{x}$-ray structure factors for this sample at $42 \mathrm{GPa}$ and ambient pres. sure $(0.1 \mathrm{MPa})$. These data show that increasing pressure pr duces a large shift in $Q$ and $a$ large decrea $\theta$ in the intensity of the first sharp diffraction peak at $\mathbf{Q}$ $\approx 1.5 \mathrm{~A}^{-1}$. By fitting Monte Carlo simulations to these data, they have determined that there is an increase in the Si coordina. tion with compression of $\mathrm{SiO}_{2}$ glass to 42 GPa. This is an exainple of a pressure. induced structural transformation in a glass. C. Meade (Carnegie Institution of Washington).
In a separate study, Skelton et al. from NRL, in collaboration with Finger et al. from CIW, obtained energy dispersive diffraction data from a cylindrical single crystal of Bi having a diameter of only $\approx 0.067 \mu \mathrm{m}$. Provisional refinement of these data reveal a new phase of bismuth, $\mathrm{Bi} \cdot \mathrm{X}$, with an fcc lattice $(a=10.65 \pm 0.05 \AA)$ belonging to the space group F-43m.

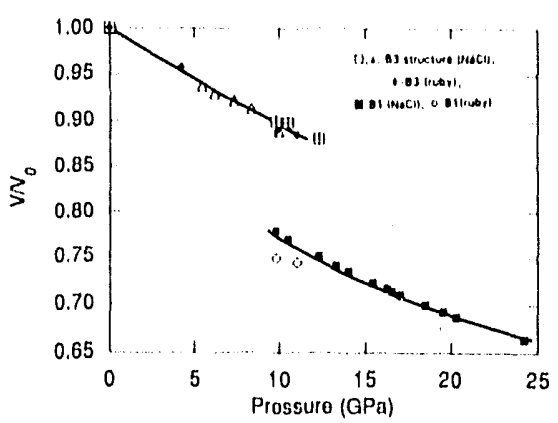

Fig. 14. The equation of state of $\mathrm{Zn}_{1-x} \mathrm{Fe}_{\mathrm{x}} \mathrm{Se}(\mathrm{x}=0.17)$ measured at X17C. Symbols indicate the $\mathrm{Zn}_{1-x} \mathrm{Fe}_{x}$ Se structure and the internal pressure standard $(\mathrm{NaC})$ or ruby). A phase transition occurs at about $10 \mathrm{GPa}$. 


\section{LITHOGRAPHY, MICROSCOPY, AND TOMOGRAPHY}

\section{G.P. Williams \\ NSLS}

\section{Lithography}

The three operating $x$-ray lithography beamlines continued active programs during FY 1991. Beamlines U2 (IBM) and U6 (IBM) are primarily concerned with proxim. ity printing although some of the radiation induced effects are of general interest. U13UB (AT\&T Bell Labs) is primarily concerned with projection printing.

U2 operated reliably with the Karl-Suss stepper being used for several production runs in conjunction with personnel from Grumman Aerospace Corporation.

U6 was used in several different studies, all of which can be classified as radiation induced effects. These included studies of mask distortions, of new resists, and of radiation damage effects to the circuits being printed. Specific examples are the effects on the emitter gain of bipolar transistors and the susceptibilities of different gate dielectrics and other insulating elements to degradation due to the creation of residual electron traps. The effects of annealing on radiation induced damage was also a part of the study, and it was found that in general, induced charged or bipolar traps in the materials anneal out well compared with neutral traps. Thus it is desirable to choose materials which are less prone to the creation of neutral traps.

At U13UB several studies of the physics of reflective optics and diffraction limited imaging took place. Two instruments, a Schwartzschild reduction optic and an Offner 1:1 ring field system, were used to print 0.1 micron lines and spaces using both transmission and reflective masks (see Fig. 15).

\section{Schwarzschild Camera Images in PMMA Using Patterned Reflection Masks}

Etched Ge Absorber

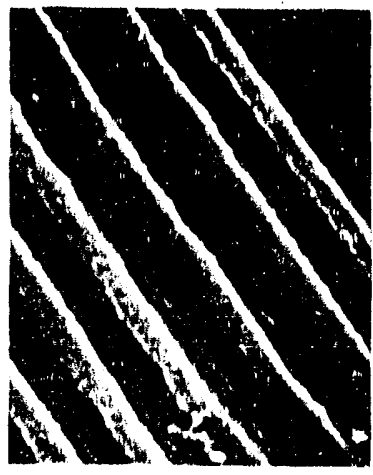

The reflective masks are part of the program involving multi. layers which will also be applied to reducing the operating wavelength of the ring field camera systum from its present value of $46 \mathrm{~nm}$ to $14 \mathrm{~nm}$.

Multilayers were also studied at U8 (IBM) for use in a soft $x$-ray telescope which was used by collaborators at the HarvardSmithsonian Center for Astro. physics to photograph the sun.
Fig. 15 The Advanced Lithography Research Department of AT\&T Bell Li.'Joratories is involved in an experimental program on Bcamline U13UB to determine the feasibility of using soft $x$-rays at wavelengths near $14 \mathrm{~nm}$ to carry out advanced projection lithography. It has been suggested that soft x-ray projection printing can be accomplished using a reflecting mask fabricated on a rugged, stable substrate. The substrate must exhibit minimal thermal distortion, since the power incident on the mask may be hundreds of milliwatts. This gives reflecting masks a great potential advantage over the membrane masks currently used in $x$-ray proximity printing.

The U13UB researchers have for the first time compared the performance of Mo/Si multjlayer coated reflection masks on rugged, stable substrates to that of a transmission mask using a 20:1 reduction Schwarzschild optic at $14 \mathrm{~nm}$. They found that there is no observable difference among reflection masks patterned using different fabrication techniques, as shown in these scanning electron microscope photographs of images of 0.1 micron lines and spaces in a $60 \mathrm{~nm}$ thick layer of PMMA resist. (a,b) Printed from reflecting masks patterned hy absorbing layers deposited on top of a multilayer reflector. (c) Printed from a reflective mask patterned by reactive ion removal of reflective coating. There is no observ. able difference between reflection and transmission mask results. R. Freeman, J. Bjorkholm, D. Tennant, L. Eichner, 'T. Jewell, A. MacDowell, L. Szeto, D. Taylor, W. Waskiewic\%, D. White, D. Windt, and O. Wood (AT\&T Bell Labs). 


\section{Microscopy}

The x-ray microscopy program at $\mathrm{X} 1 \mathrm{~A}$ saw several developments during FY 1991, both in terms of technique, development and application. 'The scanning transmission microscope was upgraded to a resolution of $45 \mathrm{~nm}$ using a new zone plate and scanning stage (see Fig. 16).

Some of the biological samples imaged with this improved setup hav $\rightarrow$ been muscle, calcium deposits in human cartilage, zymogen granules, in which it was possible to estimate the protein content, and chromosomes (see Fig. 17).

Not only can the beam be almost routinely focused to these dimensions, but using the high brightness, it was possible for the first time to detect luminescence from a sample as the micro-focused beam was scanned over it (see Fig. 18).

In another new application, it proved possible to tune the photon energy to the energy corresponding to transitions from the 1 s level to the aromatic $2 \pi^{*}$ of carbon and to other nearby final states in a polymer mixture, and so image carbon in a specific chemical state. These experiments involve adjusting the focus of the sample since the zone plate images different wavelengths at different distances. Finally, a series of experiments began in which a series of new detectors were tested with the aim of doing phase contrast. imaging. In a demonstration of the application of $x$-ray microscopy, a scanning photoelectron microscope picture was successfully taken of a microelectronic

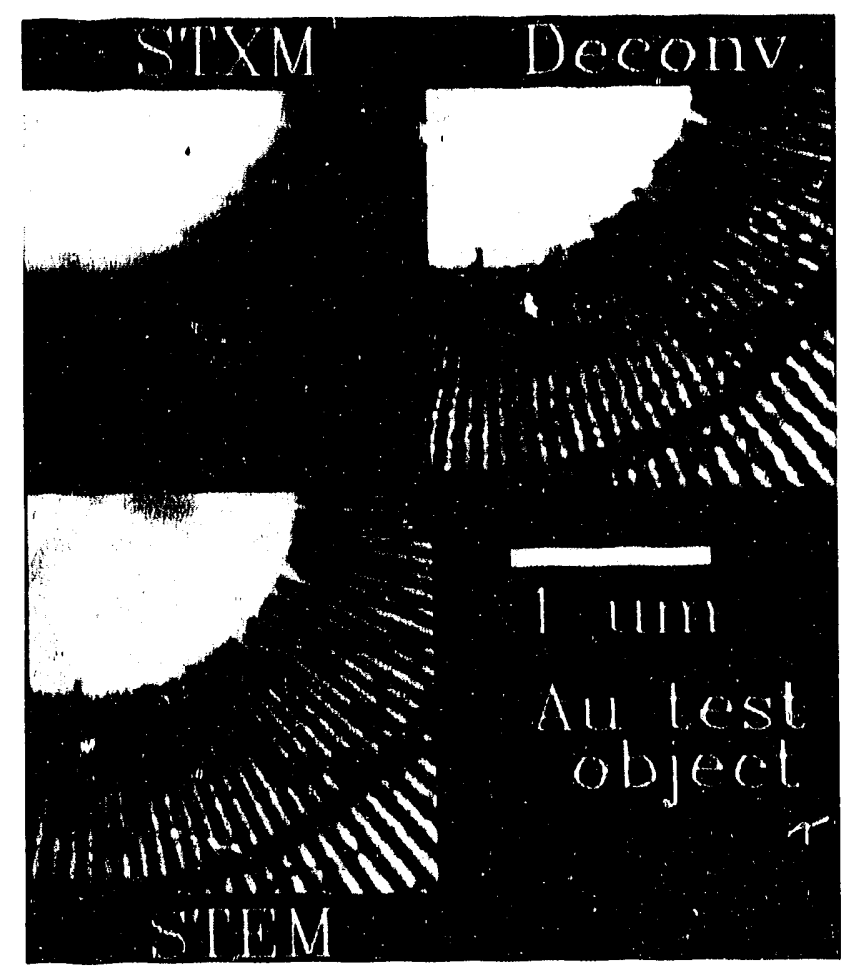

Fig. 16 Three $2 \times 2 \mu \mathrm{m}^{2}$ images of a Au test object, as viewed in the X1A Scanning Transmission X.ray Microscope (STXM), the STXM with deconvolution, and with a Scanning Transmission Electron Microscope (STEM). C. Jacobsen, S. Williams, J. Kirz, X. Zhang (SUNY at Stony Brook), E. Anderson (LBL), M. T, Browne, C. J. Buckley (King's College, London), D. Kern (IBM), and M. Rivers (U. Chicago).

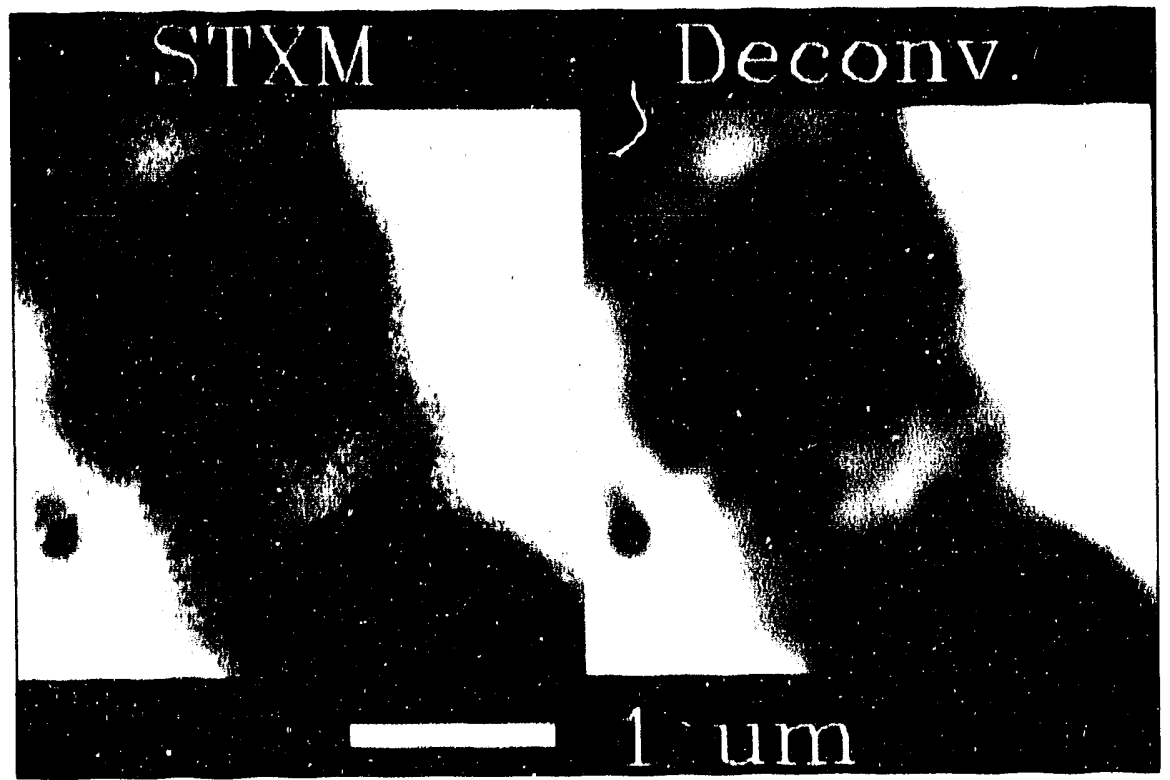

Fig. 17 X1A STXM image of a Vicia faba M chromosome kinetochore. The goal of this work is to quantify the mass per unit length versus chromosome type and to understand radiation damage effects on chromosome structure. At left is shown the original $23 \mathrm{~nm}$ pixel size S'TXM image, which was taken over 4 minutes for a transmitted flux of 1300 photons/ pixel in the transparent background regions. At right is shown the image as processed by a deconvolution filter. The deconvolution appears to restore the proper contrast of high resolution features, and pixel-t.j-pixel shot noise has been reduced by image oversampling and application of a Wiener filter. S. Williams, C. Jacobsen, J. Kirz (SUNY at Stony Brook), S. Lamm, and J, Van't Hof (BNL Biology Dept). 
device at a one-dimensional resolu* tion of $100 \mathrm{~nm}$ and a two dimensional resolution of $400 \mathrm{~nm}$. The contrast in the image was due to the different elements of which the circuit was composed and their different chemical states (see Fig. 19).

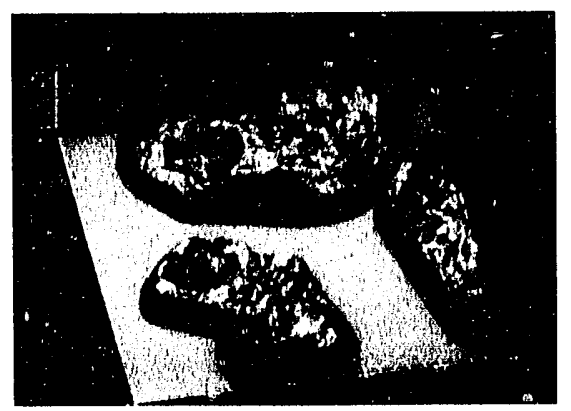

Fig. 18 Scanning luminescence x-ray $\mathrm{mi}$ croscopy (SLXM) is based on the use of the very small focused probe of a scanning $x$. ray microscope to stimulate visible light emission from dyes usually used in conven. tional fluorescence microscopy. Using a soft x-ray undulator source and a Fresnel zone plate to produce a focused $x$-ray probe, researchers at $\mathrm{X} \perp \mathrm{A}$ have obtained 50-75 $\mathrm{nm}$ resolution images of the $\mathrm{ZnS}$. based P31 phosphor grains shown here. The resolution was limited not by the focused $x$-ray probe (they have resolved 36 nm structures in the Scanning Transmission X-ray Microprobe (STXM)) but by dark noise and low net efficiency $(70 \%)$ in the luminescence detection system used for this first investigation. This technique should make possible the imaging of dyetagged sites of biochemical activity at 20$60 \mathrm{~nm}$ resolution in wet, unsectioned, and perhaps even initially living cells. A possible extension of the technique for 3D imaging at the transverse resolution of the $x$-ray microscope is described, wheie visible light collection optics might be used to obtain $<500 \mathrm{~nm}$ axial resolution. C. Jacnbsen, $\mathrm{S}$. Lindaas, S. Williams, and X. "'..ung (SUNY at Stony Brook).

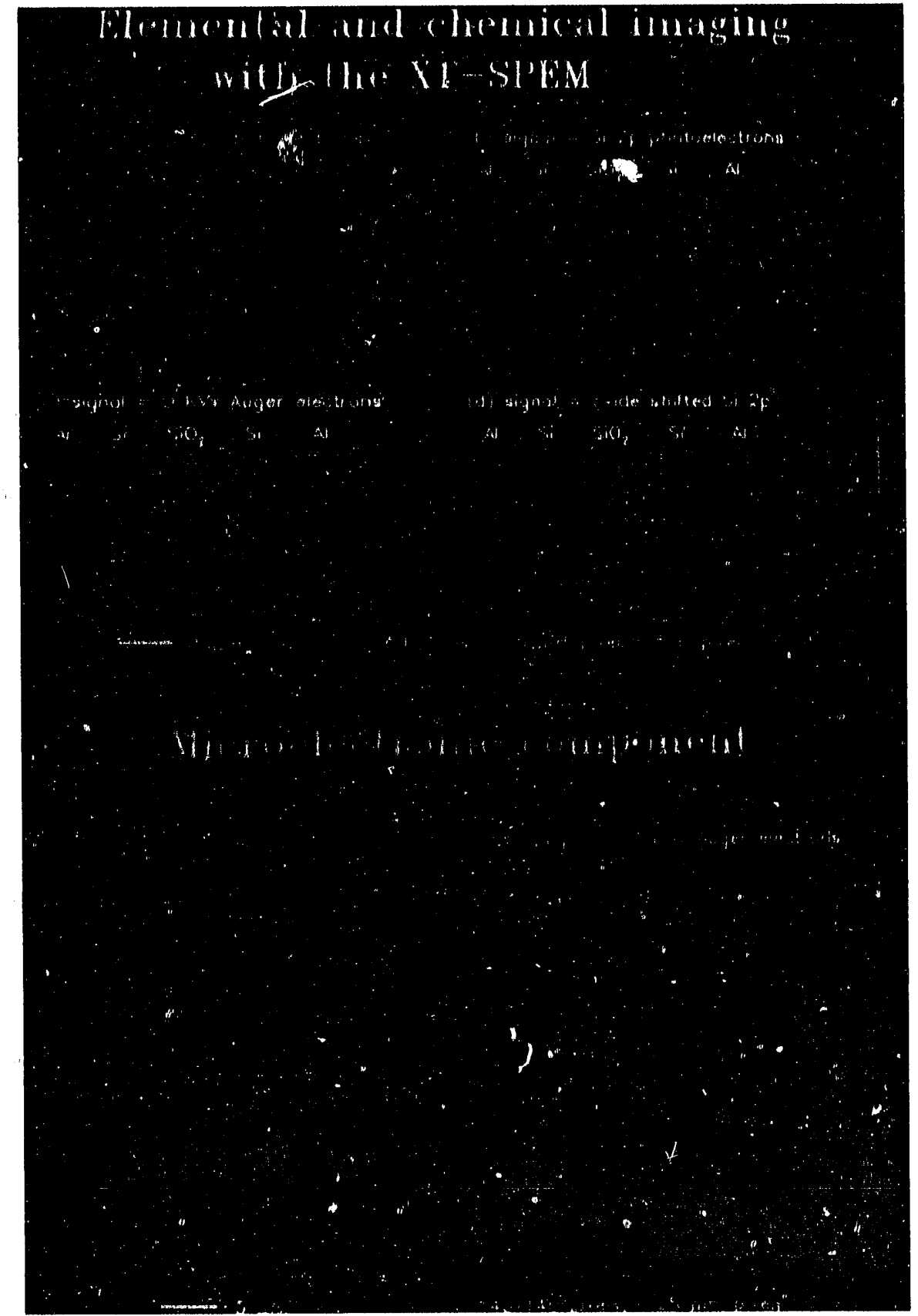

Fig. 19 Top: Chemical and elemental composition of a microfabricated device as determined with the X1A Scanning Photoemission Microscope (SPEM). Elemental alumi. num (a), silicon (b), and oxygen (c) are identified and mapped by selecting characteristic electron energies associated with each element in turn. Silicon atoms bound in silicon dioxide molecules (d) have an energy that is slightly shifted from the pure silicon level. In this way, two different chemical states of silicon have thus been distinguished. Bottom: Additionally, the instrument can acquire two images simultaneously by monitoring both (a) the sam. ple current (representative of the total electron yield (TY)) and (b) the intensity of the electron energy analyzer (in this case the oxygen KVV Auger yield). Areas with low oxygen concentration appear dark in (b) but bright in (a). Note that there are streak-like features near the bottom of (a) which are not present in (b), which is attributed to changes in work function of sub-surface material. Some microelectronic gates are imaged in the top right corner of (a) and (b), although the gates themselves cannot be resolved owing to insufficient two-dimensional resolution $(\approx 0.4 \mu \mathrm{m})$. The photon energy is $690 \mathrm{eV}$, the array size is $140 \times 140$, with a pixel size of $0.25 \mu \mathrm{m}$ and a dwoll time to $200 \mathrm{~ms}$ (images are median filtered). H. Ade, J. Kirz (SUNY at Stony Brook), E. Johnson, S. Hulbert (NSLS), E. Anderson (LBL), and D. Kern (IBM). 


\section{Tomography}

Several interesting images were taken at X2 (Exxon), X23 (NIST), and $X 26$. X2 researchers recorded images of calcium deposits in cartilage tissue (see Fig. 20), images of the spatial distribution of metal atoms in a supported catalyst system, and a study of the settling of particles in a heavy oil.

Fig. 20 Microtomography investigation of the spatial distribution of calcium deposits in cartilage tissue at X2B. The sample was mounted in an epoxy substrate and cut to a cube approximately $1.5 \mathrm{~mm}$ on an edge, and was imaged at $h v \approx 15 \mathrm{keV}$. Despite the low optical depth of the epoxy and tissue material, the relatively large mineral deposits required the high photon energy in order for them to be penetrated with sufficient signal-to-noise to permit reconstructions. The relative size and orientations of the deposits was sought. It is clear from the data that the Ca deposits varied up to sizes in excess of $150 \mu \mathrm{m}$. In addition, the roughly spherical particles appear to be arranged in a well defined pattern, much like beads on a string. Further experiments are planned to investigate the location of deposits relative to physical features such as the neighboring bone tissue. This figure is a volume rendering of the cartilage sample showing the tissue matrix (lighter color) and the $\mathrm{Ca}$ deposits, which are the dark nodules which appear to be strung together. K. L. D'Amico, J. H. Dunsmuir, S. R. Ferguson (Exxon), and C. J. Buckley (King's College, London, UK).
Synthetic calcite was imaged at $\mathrm{X} 26 \mathrm{C}$ and compared with electron microprobe analysis. This evaluation of the usefulness of the synchrotron images will continue.

Finally, some exotic samples were imaged at X23, from crystals of triglycine sulfate, mercuric iodide and lead tin telluride grown in Spacelab III and on the Space
Shuttle to synthetic diamonds. Also studied were nonlinear optical crystals of L-arginine phosphate which have a potential application in optical devices. In all cases images of superb contrast were obtained as can be seen in the abstracts.

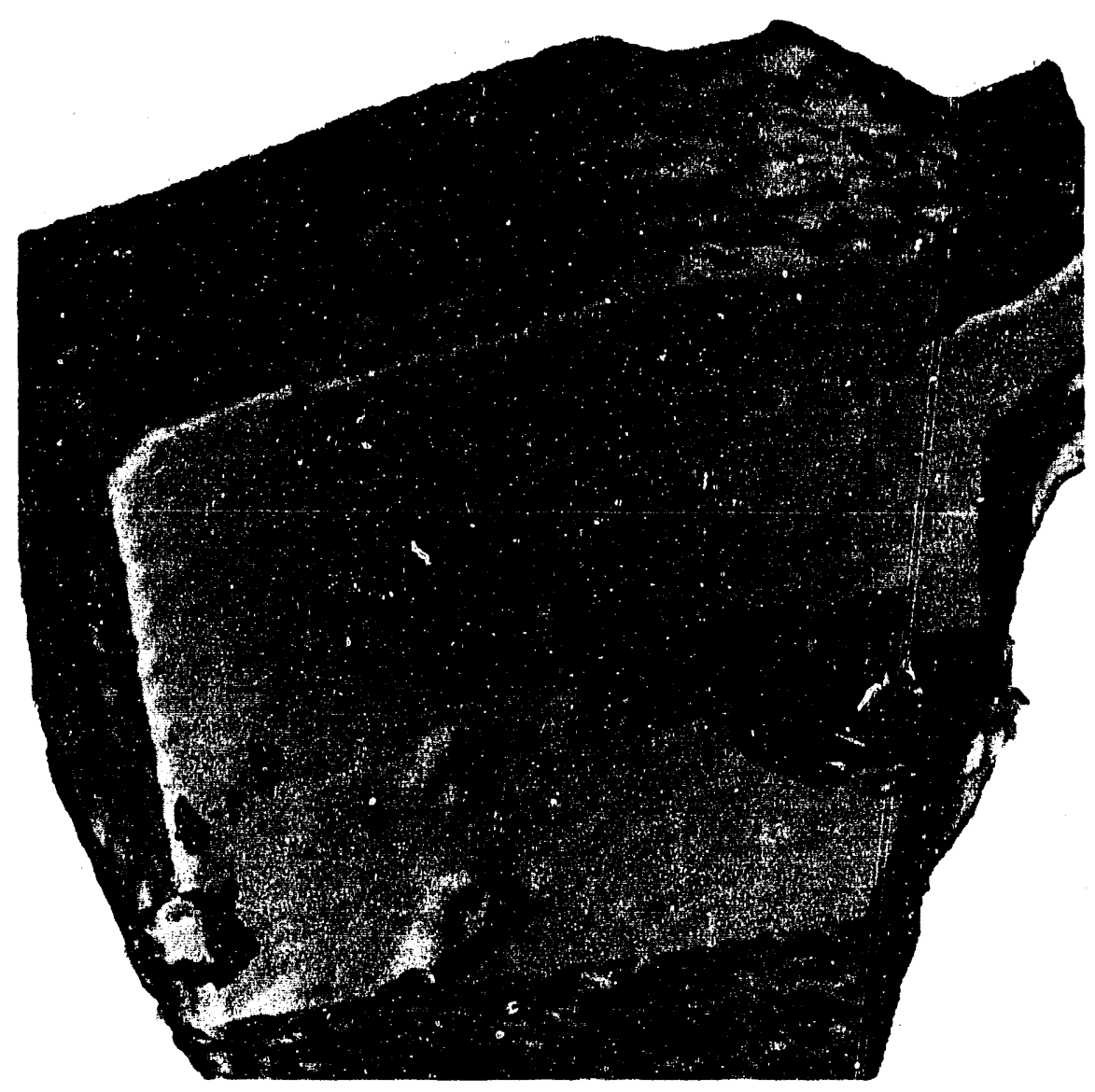




\section{NUCLEAR PHYSICS}

\section{Andrew M. Sandorfi BNL Physics Department Special Interest Group Representative}

Beamline X5, the Laser Electron Gamma Source, has completed its second full year of operations and a full year it was. Several sig. nificant improvements were made to the gamma-ray beam and to the experimental equipment for nuclear physics measurements. Many new collaborators have joined in making these improvements, and more importantly, in performing four separate major experiments during the past year. The gamma-ray beam was produced for about 160 days. Of this total, approximately $20 \%$ was used for beam and detector development. The remaining $80 \%$ of the beam time was devoted to four experiments.

L3: Photodisintegration of the deuteron at energies from 200 io $320 \mathrm{MeV}$ to study the role of the nuclear tensor force (38 days);

L4: Three body final states in the photodisintegration of ${ }^{3} \mathrm{He}$ to determine the importance of three body forces (48 days);

L5: Neutral pion photoproduction on the proton to determine the deformation of the delta isobar, the first excited state of the nucleon (18 days);

L6: Three body photodisintegration of the deuteron to determine properties of the nucleon-delta interaction (28 days).

Experiments L4 and L6 are part of our outside-user program and were performed in collaboration with a group from Renssellaer Polytechnic Institute (RPI).

We have replaced the laser with a higher power version, so that it now delivers 7 watts of power in the ultraxiolet from $333 \mathrm{~nm}$ to 364 $\mathrm{nm}$, which produces gamma-rays up to $319 \mathrm{MeV}$, and 2 watts deeper in the ultraviolet from $300 \mathrm{~nm}$ to $336 \mathrm{rm}$, which produces gammarays up to $349 \mathrm{~N}: \mathrm{V}$. Operation at this higher energy is a new capability, and it will become a common operating mode for LEGS. The higher energy allows us to make measurements on and above the delta resonance, which will significantly enhance new measurements of the delta deformation planned for the coming year.

A new cryogenic target, capable of liquefying ${ }^{1} \mathrm{H},{ }^{2} \mathrm{H},{ }^{3} \mathrm{He}$, and ${ }^{4} \mathrm{He}$, was constructed and installed in the target area, principally by collaborators from the University of Rome and RPI (Annalisa D'Angelo, Carlo Schaerf, and Kamran Vaziri). An array of 48 plastic scintillator bars, $1.5 \mathrm{me}$ ters long, was mounted above the target by the University of Virginia (Richard Sealock, Steven Thornton, Richard Lindgren, and Cole Smith). The entire detector and target stand is mounted on rails so that it can be removed intact from the beam to allow experiments to be run in alternation, with minimal disassembly.

Keith Mize, the first graduate student to work at LEGS, recejved his $\mathrm{PhD}$ degree from the University of South Carolina this year. His thesis work (experiment L1) on the effect of the nuclear tensor force on beam-polarization observables in deuteron photodisintegration, was published in Physical Review Letters in September. Three other graduate students, all from Renssellaer Polytechnic Institute, have finished their thesis experiments at LEGS this year (experiments L4 and L6) and are now immersed in the analysis of their data. A visiting scientist from Grenoble, Dominique Rebreyend, is spending the year at LEGS to learn details of the construction and operation of the Compton backscattered gammaray bean, as well as collaborate in experim 'nts. He has been working at ERSF on the design of a similar gamma-ray facility called GRAAL.

The major effort at beamline X5 for the next year will be a series of measurements to determine the deformation of the first excited state of the nucleon, the delta resonance at $320 \mathrm{MeV}$. Essentially all quark models of the nucleon invoke a tensor force between the quarks. This non-central force breaks the otherwise spherical symmetry of the nucleon, resulting in a deformation and static quadrupole moment for the delta. The delta is excited mainly by M1 photons, but if it has a quadrupole deformation, then it can also be excited by an E2 photon. The dominant decay channel is pion emission. Sensitivity to the small E2 component is enhanced by observing the photo-pion production with linearly polarized photons and determining the ratio $\frac{d \sigma 11}{d \sigma \perp}$ 
of cross sections with the polarization parallel to and perpendicular to the reaction plane. Preliminary results of these measurements are shown in Fig. 21, along with previous data and the results of two independent calculations, each with and without a quadrupole deformation of the delta. The calculation of Davidson, et. al., which have approximately the correct energy dependence, can be brought into agreement with the data for a mixing ratio of about $4 \%$. This would imply a larger deformation than expected in most models. Unfortunately, the agreement is not as good at other reaction angles. New experiments at X5 will provide improved determination of other parameters upon which the calculations depend, as well as further reducing uncertainties in polarization observables such as those shown in Fig. 21.

Nuclear physics research on beamline $\lambda_{2}$ is performed by a collaboration of physicists from BNL, INFN Frascati, Universita di Roma, University of South Carolina, University of Virginia, Virginia Polytechnic Institute and State University, Tel Aviv University, and Renssellaer Polytechnic Institute.

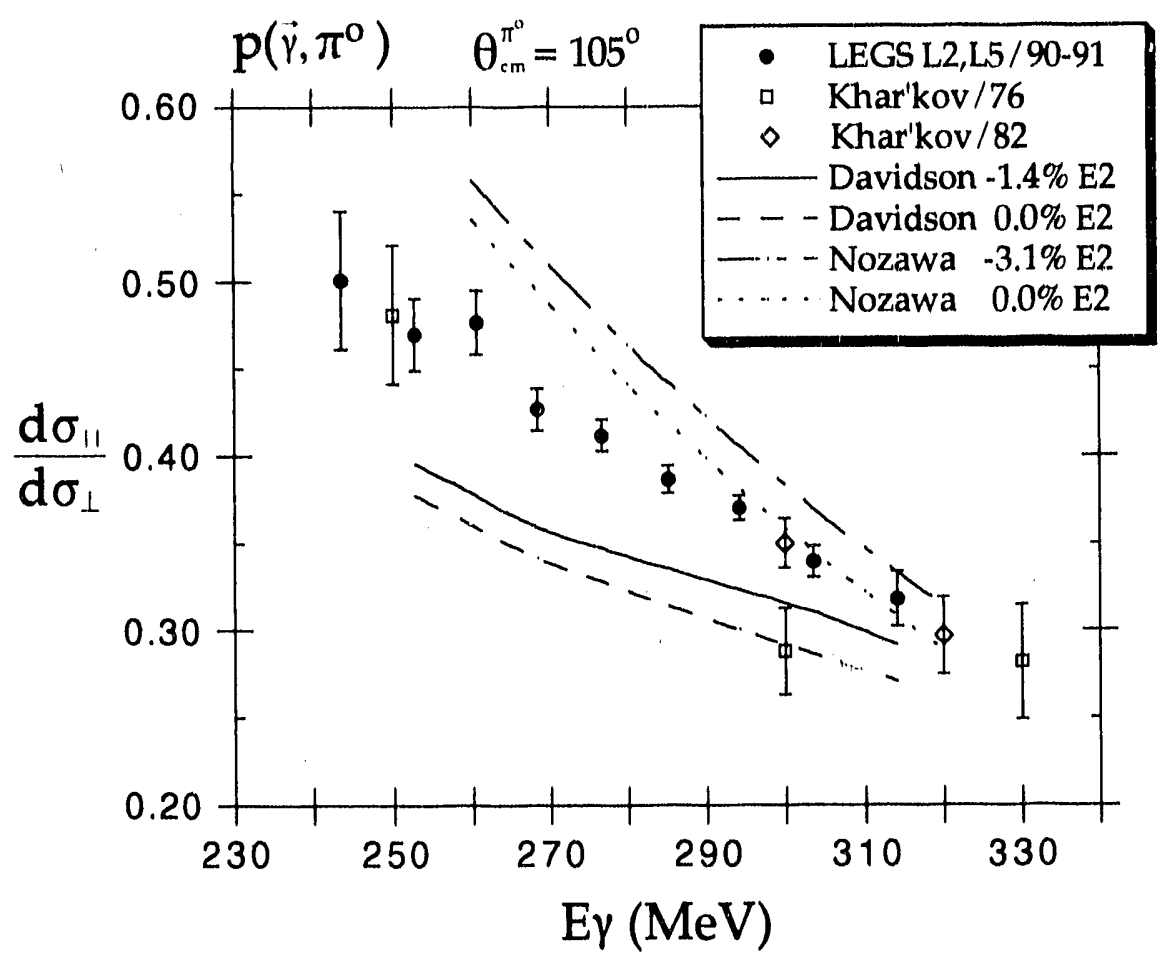

Fig. 21. Ratio of cross sections for production of neutral pions by linearly polarized photons on hydrogen with the incident photon polarization parallel and perpendicular to the reacton plane. The solid circles are the average of two separate experiments, L2 and L5, made at LEGS (X5). The calculations demonstrate the sensitivity of the measurement to the quadrupole deformation of the delta resonance. 


\section{UV \\ PHOTOEMISSION \\ AND \\ SURFACE SCIENCE}

\section{Chi-Chang Kao \\ NSLS}

\section{(1) Angle-resolved photoemission}

Angle-resolved photoemission spectroscopy continues to be one of the most important experimental techniques in the study of electronic structure of materials. During the past year, angleresolved photoemission has been used to study the electronic band structures of a variety of bulk solids (U3, U7B, U12B, U16A), surfaces (U4A, U7B, U16A), ad. sorbate covered surfaces (U4A), thin metal overlayers (U7B, U16A), metal-semiconductor interfaces (U12B), as well as high temperature superconductors and related materials (U3). Spinresolved angle-resolved photoemission also continues to provide important information on surface and interface magnetism. The following are a few representative examples:

(1.1) The bulk electronic band structures of two experimentally very demanding systems, $\mathrm{Li}$ (U12B) and Gd (U16A), were measured.

Along the $\Gamma->\mathrm{N}$ direction of the Brillouin zone of BCC Li, both the bandwidth and the shape of the dispersion were found to deviate strongly from the behavior predicted by the free electron model.
Better agreement with the experimental results was achieved by taking into account the exchangecorrelation in the framework of the local density approximation (LDA). Almost exact agreement is achieved by further including the quasiparticle self-energy (see Fig. 22).

In the bulk band structure of Gd(0001), along the $\Gamma->$ A direction, two bulk band states with predominantly 6 s character were observed. The splitting between the two states is int orpreted as a manifestation of indirect exchange between the nonoverlapping $4 \mathrm{f}$ states via conduction electrons. The temperature dependence of the splitting yields a critical exponent $\beta$ of 0.378 , which agrees very well with the results obtained from the magnetization measurement of bulk Gd (see Fig. 23).

(1.2) Spin-resolved angle-resolved photoemission was used to study the magnetic and electronic structure of $\mathrm{Fe} / \mathrm{Ag}$ interfaces. A spinpolarized interfa state was observed in the $\mathrm{Ag} / \mathrm{Fe}(001)$ system. It shows a distinct quantum size effect, a single new electronic state being observed for each layer, up to three atomic layers. These results may have important implications in the understanding of the oscillatory indirect exchange coupling between two ferromagnetic layers separated by thin layers of nonmagnetic transition metals (see Fig 24).

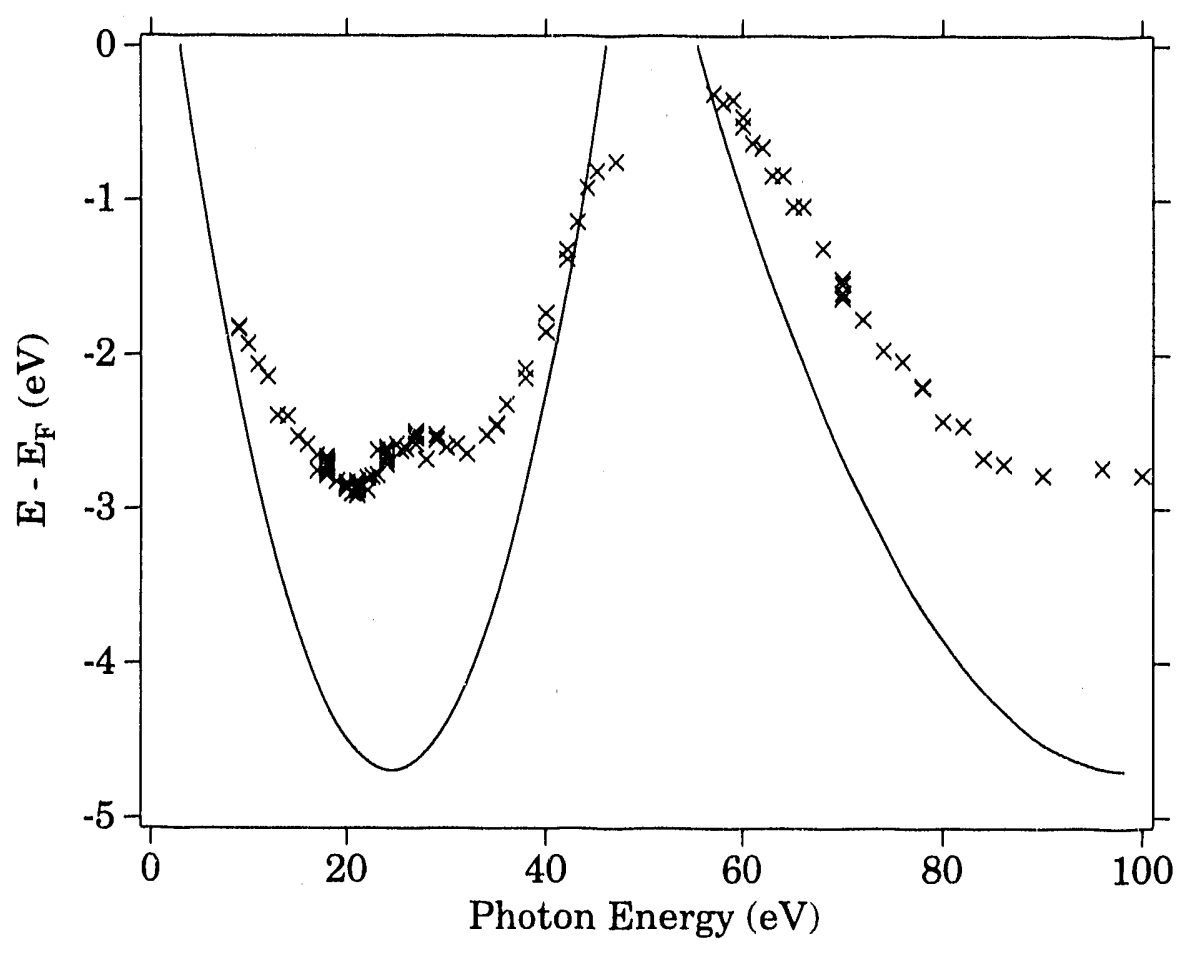

Fig. 22 Bulk band structure of BCC Li along the $\Gamma->$ N direction in the bulk Brillouin zone. Measured initial state energy (crosses) and free electron metal dispersion (solid line) are shown. The LDA bandwidth $(3.45 \mathrm{eV})$ is closer to the experimental value $(2.85 \mathrm{eV})$ than the free electron model predicts, and the quasiparticle bandwidth in the $\mathrm{GW}$ approximation $(2.84 \mathrm{eV})$ shows nearly exact agreement with the experiment. G. M. Watson, X. Y. Liu, and E. W. Plummer (University of Pennsylvania). 
Fig. 23 (a) Norrs al emission electron energy distribution curves (EDCs) for $\mathrm{Gd}(0001)$ at a photon energy $\mathrm{h} \nu=38 \mathrm{eV}$ as a function of temperature in the range $0.35 \mathrm{~T}_{\mathrm{c}}<\mathrm{T}<\mathrm{T}_{\mathrm{c}}$, where the Curie temperature $\mathrm{T}_{\mathrm{c}}=293 \mathrm{~K}$. (b) Temperature dependence of the exchange splitting of the $\sim 1.6 \mathrm{VV}$ binding energy peak in (a). The solid line is $\left(1-T / T_{c}\right)^{\beta}$, where the best fit critical exponent $\beta=0.378$ agrees well with bulk magnetization measurements of Gd. B. Kim, A. B. Andrews, J. L. Erskine (U. Texas at Austin), K. Kim, and B. N. Harmon (Iowa State U.).

Fig. 24 Spin polarized (a) and spin integrated (b) angle-resolved photoemission spectra taken using beamline U5U at normal emission, $\mathrm{h} \nu=52 \mathrm{eV}$, and $70^{\circ}$ angle of incidence, for $\mathrm{Fe}(001)$ epitaxially covered with $0,1,2$, and 3 monolayers of $\mathrm{Ag}$. The $\mathrm{Fe}(001)$ surface state in the clean $\mathrm{Fe}$ spectrum (indicated by arrow) evolves into the $\mathrm{Ag} / \mathrm{Fe}$ interface state in the 1,2 , and 3 monolayer $\mathrm{Ag} / \mathrm{Fe}$ spectra (indicated by arrows). N. B. Brookes, Y. Chang, A. Clarke, P. D. Johnson, and M. Weinert (BNL Physics Dept.). See Phys. Rev. Let.t. 67, 354 (i991).
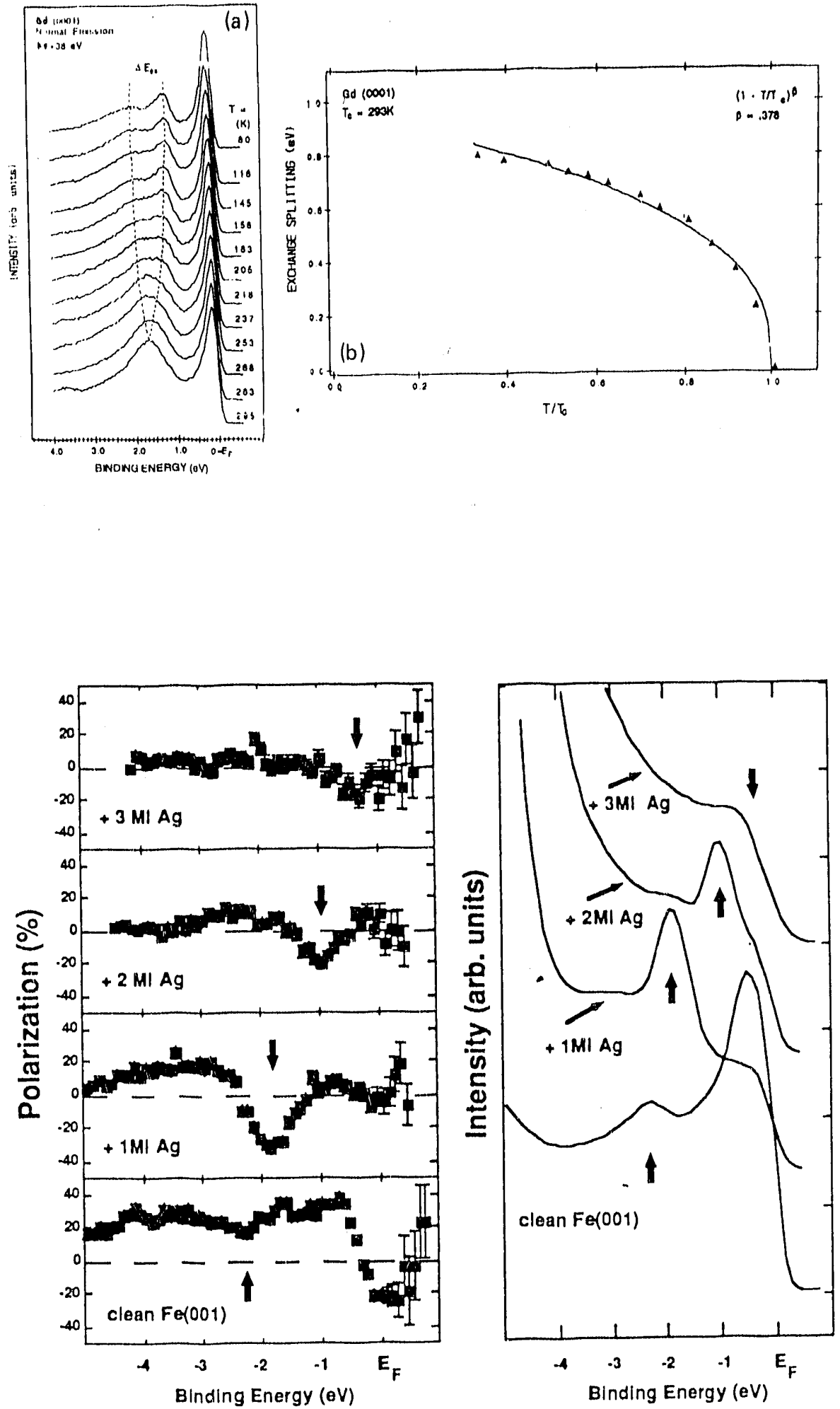


\section{(2) Valence band photoemission}

There also have been a large number of angle-integrated valence band photoemission experiments reported. Among them, one of the most important developments is the extension of resonant photoemission to the soft $x$-ray region (U4B). There is also an increase in the use of the Cooper minimum in the photo-absorption cross section to selectively probe the valence band partial density of states of individual components in alloys (U4A,U7B). Finally, high resolution photoemission has been used to study the temperature dependent Kondo resonances in heavy Fermion systems (U3,U4B).

(2.1) Resonant photoemission has long been an invaluable tool in the study of the electronic structure of correlated systems due to its capability to identify different electronic configurations in the valence band photoemission spectra. In their study of $\mathrm{CuO}$, the authors demonstrated that for $3 \mathrm{~d}$ transition metal systems, carrying out the measurement near the $2 p$ absorption edge instead of the more commonly used $3 p$ absorption edge has several advantages which simplify the data interpretation. Among these advantages are larger resonant enhancement due to stronger absorption and larger spin-orbit splitting compared to the lifetime width of the core hole (see Fig. 25).

(2.2) The prevailing theory of photoemission of heavy Fermion compounds is the Konclo impurity theory, which predicts a Kondo resonance in the spectrum located an energy $\mathrm{kT}_{\mathrm{K}}\left(\mathrm{T}_{\mathrm{K}}\right.$ is the Kondo

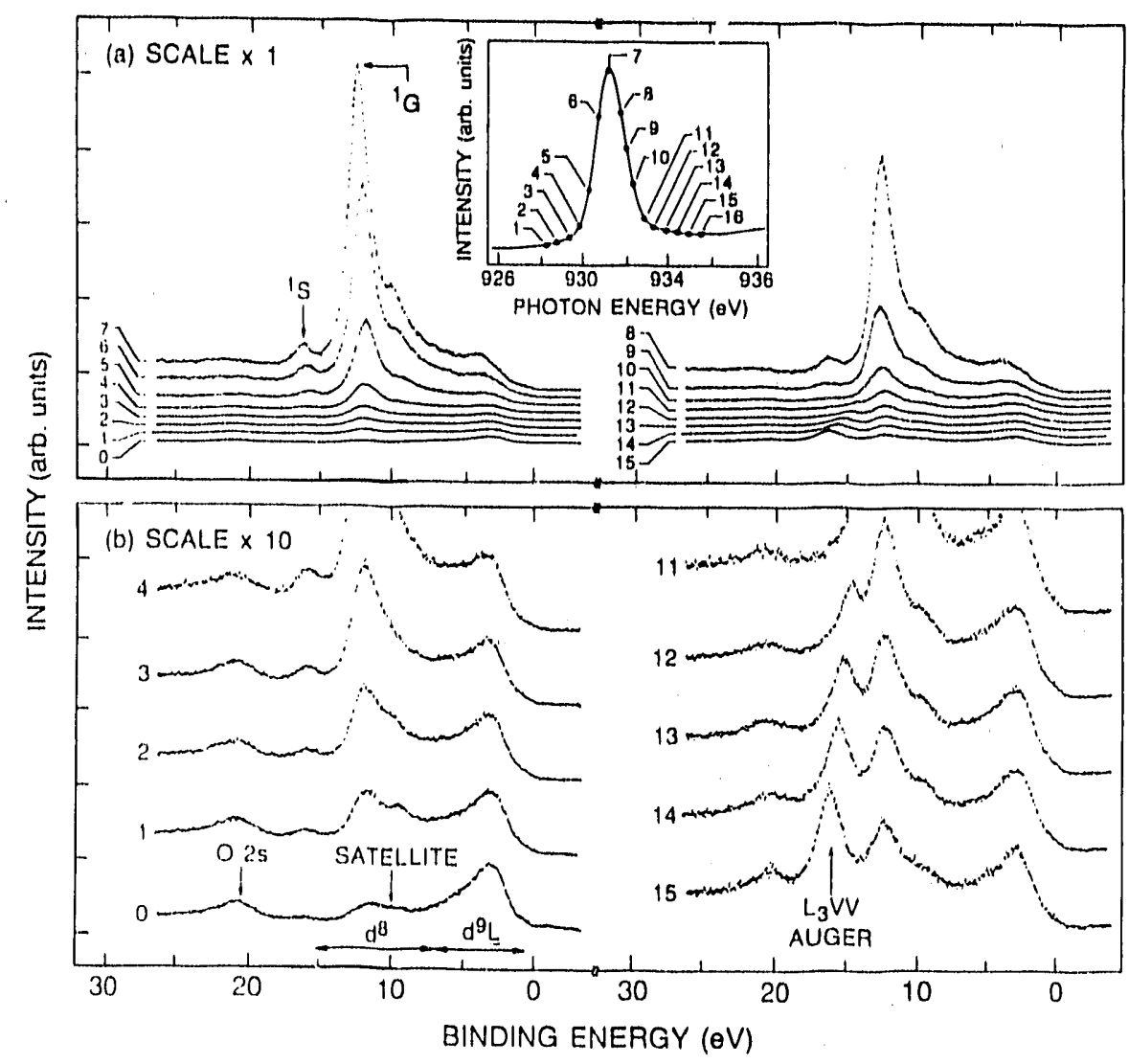

Fig. 25 Giant $\mathrm{Cu} 2 \mathrm{p}$ resonances in $\mathrm{CuO}$ valence band photoemission. (a) Valence band photoer.aission spectra of $\mathrm{CuO}$ at photon energies in the vicinity of the $\mathrm{Cu} 2 \mathrm{p}_{3 / 2}$ absorption edge, exhibiting a giant ( $\sim 100$ fold) resonant effect. (b) Spectra from (a) plotted on an expanded vertical scale, which provide an unambiguous identification of the local $\mathrm{Cu} 3 \mathrm{~d}^{8}$ and $3 d^{9} \underline{\mathrm{L}}$ configurations. This provides conclusive evidence for the charge-transfer nature (as opposed to Mott-hisbbardlike nature) of the insulating gap in the electronic structure of $\mathrm{CuO}$. L. H. Tjeng, C. T. Chen, P. Rudolf, F. Sette (AT\&T Bell Laboratories), and J. Ghijsen (FUNDP, LISE, Belgiunn). See Phys, Rev. Lett. 67, 501 (1991).

temperature) above the Fermi level for the single $4 \mathrm{f}$ electron $\mathrm{Ce}$ compounds, and $\mathrm{kT}_{\mathrm{K}}$ below the Fermi level for the single $4 \mathrm{f}$ hole $\mathrm{Yb}$ compounds. The theory also predicts that the spectral weight and width of the resonance should scale with $\mathrm{T}_{\mathrm{K}}$. Temperature dependent high resolution photoemission studies of a number of $\mathrm{Ce}$ and $\mathrm{Yb}$ compounds were carried out by the Los Alamos group at U3. Contrary to previous findings, the authors argue that the observed temperature dependence in their spectra can be fully accounted for by the temperature dependent broadening of the valence band (phonons) and of the Fermi function (see Fig. 26).

Clearly, this is one of the areas that will benefit most from the continuing efforts in the de. velopment of high resolution monochromators.

(2.3) A combined high resolution valence band photoemission and soft $x$-ray absorption study of the 


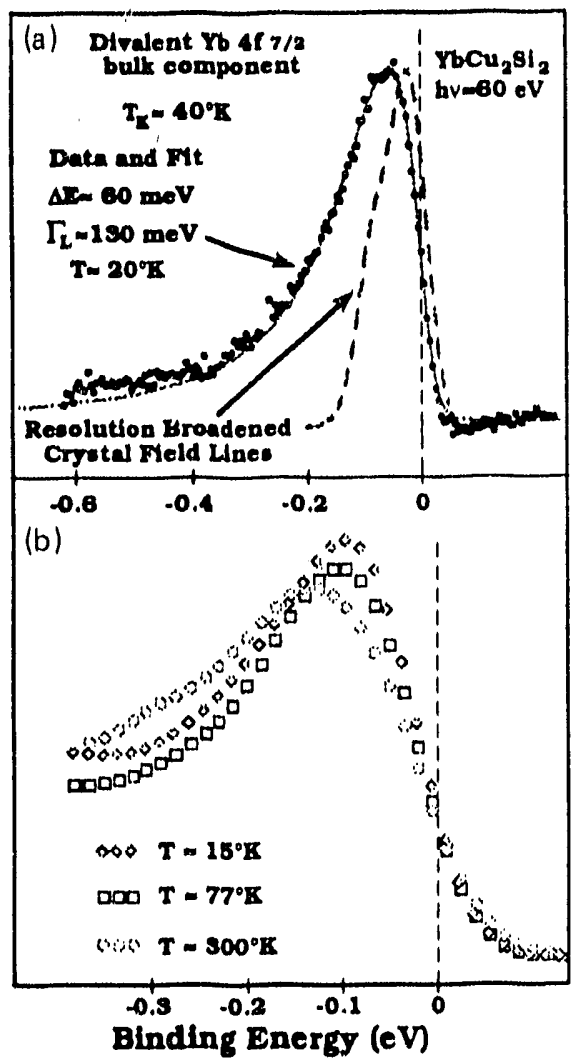

Fig. 26 (a) Photoemission spectrum of the bulk $\mathrm{Yb} 4 \mathrm{f}_{7 / 2}$ core level in $\mathrm{YbCu}_{2} \mathrm{Si}_{2}$ : data (dots), Lorentzian fit (solid line), and resolution broadened Kondo resonance model prediction (dashed line). (b) Temperature dependence of photoemission spectrum in (a). The U3C researchers conclude from this and other data that the Kondo resonance model fails in all of its major predictions: (i) the temperature dependence can be explained by Fermi function and phonon broadening, (ii) the lineshapes and widths are simple core-level-like and much broader than predicted, (iii) the valence is a conspectral weight is independent of the Kondo temperature (not shown in this figure), A. J. Arko, J. J. Joyce, J. M Lawrence, R. J. Bartlett, P. C. Canfield, Z. Fisk, and J. D. Thompson (LANL). See Phys. Rev. Lett. 68, 236 (1992).
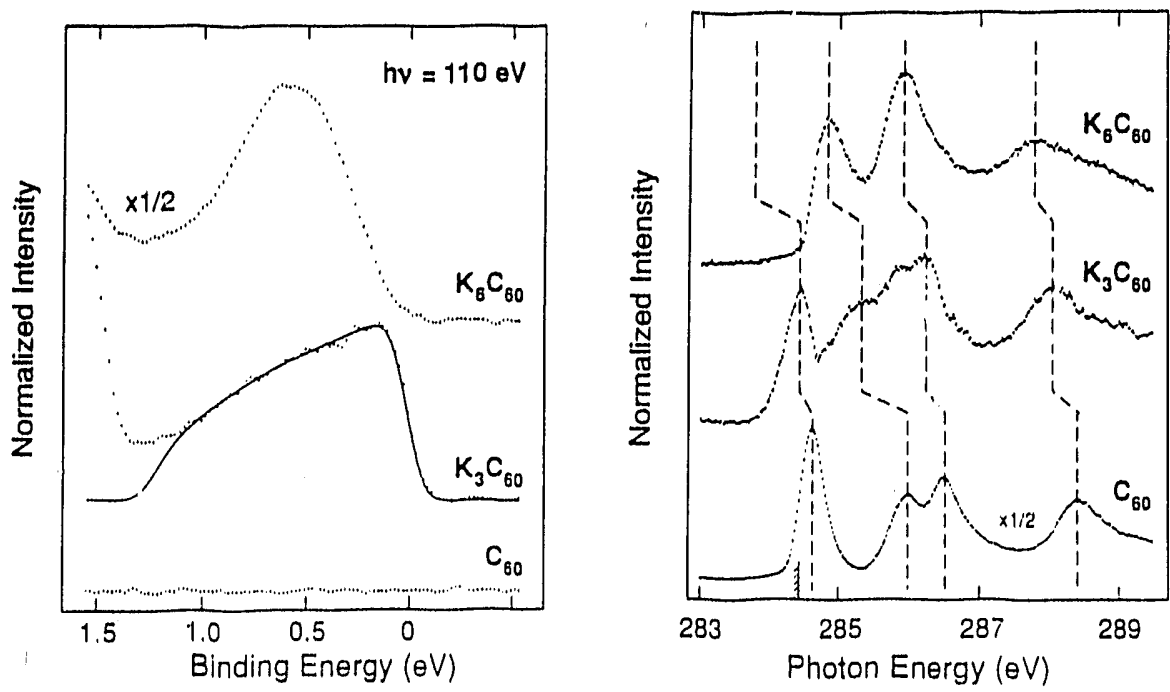
stant function of temperature, and (iv) the

electronic structure of $\mathrm{K}_{\mathrm{x}} \mathrm{C}_{60}$ was reported by U4B. The $\mathrm{K}_{\mathrm{x}} \mathrm{C}_{60}$ was found to separate in to three stable phases, $\mathrm{C}_{60}, \mathrm{~K}_{3} \mathrm{C}_{60}$, and $\mathrm{K}_{6} \mathrm{C}_{60}$, where $\mathrm{K}_{3} \mathrm{C}_{60}$ is the metallic phase responsible for superconductivity. As shown in Fig. 27, a well-defined Fermi edge is clearly observed in the $\mathrm{K}_{3} \mathrm{C}_{60}$ phase, with an occupied band width of $1.2 \mathrm{eV}$. The soft $x$-ray absorption spectra, on the other hand, show large non-rigidband shifts between the three phases, with $\mathrm{K}_{3} \mathrm{C}_{60}$ being halffilled and $\mathrm{K}_{6} \mathrm{C}_{60}$ being completely filled. These results indicate that the conduction band is formed by the lowest unoccupied molecular orbital of $\mathrm{C}_{60}$, which has predominantly $\mathrm{C} 2 \mathrm{p}$ character, and that there is strong mixing of the electronic states of $\mathrm{K}$ and $\mathrm{C}_{60}$ in the superconducting phase (see Fig. 27).

Fig. 27 Valence band photoemission (left) and $\mathrm{C} 1 \mathrm{~s}$ absorption (right) spectra from wellannealed high quality $\mathrm{K}_{\mathrm{x}} \mathrm{C}_{60}, \mathrm{x}=0,3,6$, thin films grown under ultrahigh vacuum conditions at beamline U4B. Spectra taken at other values of $x$ are found to be linear combinations of these three stable phases. The Fermi edge for the $\mathrm{K}_{3} \mathrm{C}_{60}$ film (left) is well defined and the density of states at the Fermi level suggests that this material may be a BCS-like superconductor. The $\mathrm{C} 1 \mathrm{~s}$ absorption spectra (right) exhibit large non-rigid-band shifts as a function of $\mathrm{x}$ which, combined with the anomalous occupied band width $(1.2 \mathrm{eV})$ for $\mathrm{K}_{3} \mathrm{C}_{60}$, implies that there is significant mixing of the electronic sts tes of $\mathrm{K}$ and $\mathrm{C}_{60}$ in the superconducting phase. C. T. Chen, L. H. Tjeng, P. Rudolf, G. Meigs, J. E. Rowe (AT\&T Bell Labs), J. Chen, J. P. McCauley Jr., A. B. Smith, W. J. Romanow, and E. W. Plummer (U. of Pennsylvania). See Nature, 352, 603 (1991).

\section{(3) Core level photoemission}

In core level photoemission, the sensitivity of the core level binding energy to oxidation state, chemical environment, and valence electron distribution has been used to monitor chemical reactions, and to study surface and interface electronic properties. These studies include semiconductor surface etching reactions (U8) and oxidation of tungsten and aluminum surfaces (U16B).

There were also an increasing number of high resolution core level photoemission studies performed during the last year. Higher energy resolution not only enables ever smaller core level shifts to be resolved, but it also makes possible the study of linewidth and lineshape in greater detail. For example, in a series of 
core level photoemission studies of alkali metals reported by $\mathrm{U} 4 \mathrm{~A}$, the thermal shift of core level binding energies as well as the vibrational broadening of the core levels were measured. The authors found that for $\mathrm{Na}(110)$ the width of the surface core level has a larger temperature dependence than that of the bulk core level, indicating a smaller effective Debye temperature for the surface atoms, which is consistent with an earlier proposal of the existence of a soft mode perpendicular to the surface (see Fig. 28).

\section{(4) Auger-photoelectron coincidence}

Auger-photoelectron coincidence spectroscopy (APECS) has been demonstrated to have the following unique advantages compared to ordinary photoemission: reduction of core hole lifetime broadening, elimination of uncorrelated secondary background, better surface sensitivity, and separation of overlapping spectral features.

In a study of the lineshape of the $\mathrm{Si} \mathrm{L}_{2,3} \mathrm{VV}$ Auger line of $\mathrm{SiO}_{2}$ carried out by the Rutgers university group (U14A), the major features in the coincidence spectrum are found to be reproduced quite well by an earlier calculation, as shown in Fig. 29. However, additional spectral weight is observed near $68 \mathrm{eV}$ and in the threshold region $(87 \mathrm{eV})$. The additional spectral weight near threshold is assigned to Auger transitions involving $\mathrm{Si}$ orbitals with d character, whereas the spectral weight near $68 \mathrm{eV}$ is assigned to a correlation satellite (see Fig, 29).

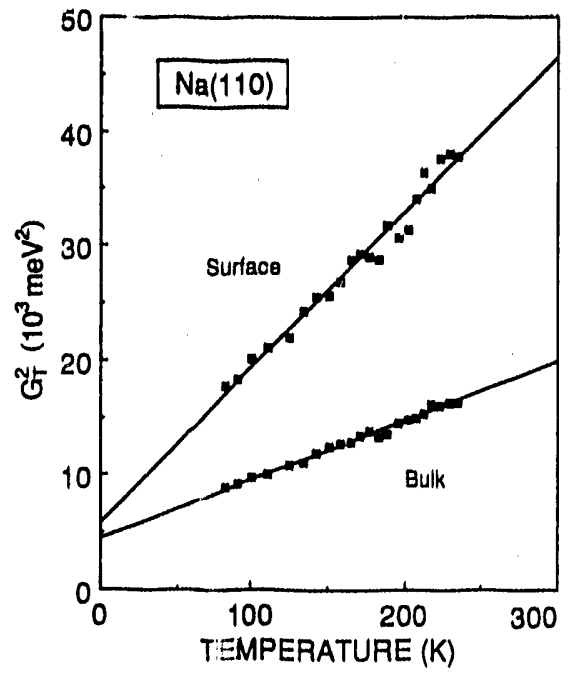

Fig. 28 Vibrational broadening of bulk and surface cort levels of $\mathrm{Na}(110)$ measured at beamline U4A. The square of the measured Gaussian widths of the surface and bulk $\mathrm{Na} 2 \mathrm{p}$ ciore levels (squares) are plotted as a function of temperature from $77 \mathrm{~K}$ to $230 \mathrm{~K}$. Above the Debye temperature, the phonon contribution to the core level width increases as $\mathrm{T}^{1 / 2}$ (solid lines). The zero temperature intercept for the bulk core level agrees well with the independently known instrumental width, indicating that inhomogeneious broadening cuntributions are small. T'he surface core level widths exhibit a small inhomogeneous contribution and, more importantly, a larger slope than the bulk core level, which im. plies that the surface atoms have a smaller effective Debye temperature than bulk atoms. This data supports the model of Jackson (Surf, Sci. 43, 431 (1974)) in which a soft mode perpendicular to the surface results in a reduced effective surface Debye temperature. D. M. Riffe, G. K. Wertheim, and P. H. Citrin (AT\&T Bell Labs).

It should be noted that the coincidence technique is essential in arriving at these assignments. For example, the alternative interpretation that the additional spectral weight near threshold is due to substoichiometric oxide was ruled out because the spectrum shown in Fig. 29 included only Auger electrons which were coincident with $2 p$ photoemitted electrons of the stoichiometric oxide.
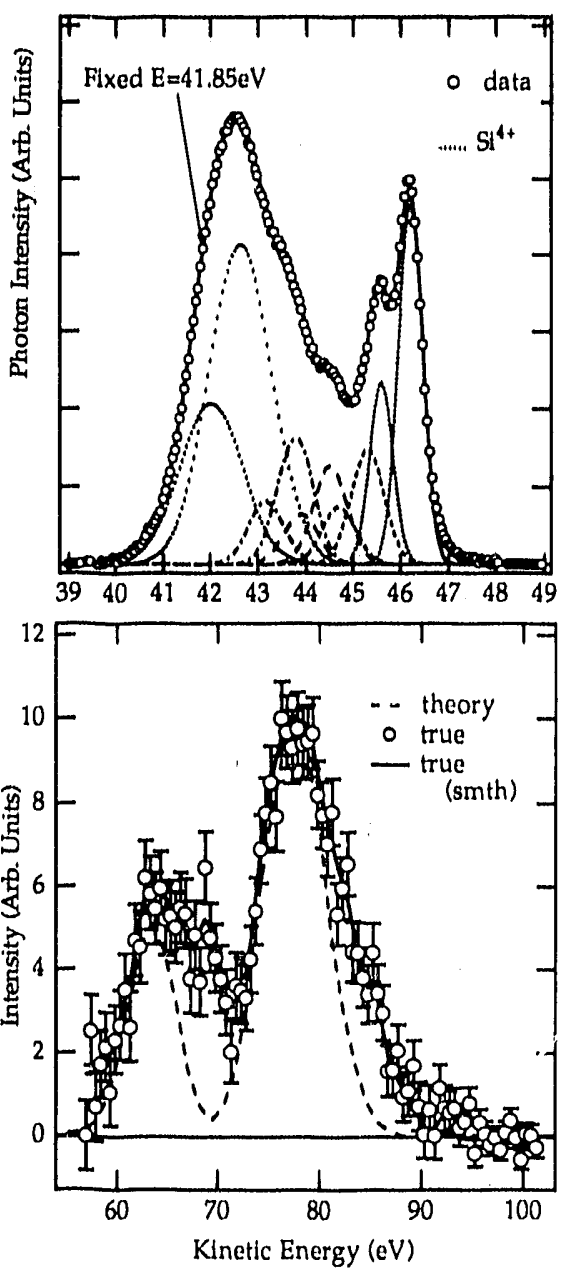

Fig. 29 Line shape of the Si $\mathrm{L}_{2} \mathrm{VV}$ Auger spectrum of stoichiometric $\mathrm{SiO}_{2}$ measured by Auger Photoelectron Coincidence Spectroscopy (APECS) using beamline U14A. Upper figure: ordinary Si 2p core level photoemission spectrum from a $7 \AA$ film of $\mathrm{SiO}_{2}$ on Si(111). The data is well fit by a series of chemically shifted Si 2 p core levels associated with substoichiometric oxides as proposed by Himpsel (Phys, Rev, B 38, 6084 (1988)). Lower figure: background subtracted Auger spectrum of stoichiometric $\mathrm{SiO}_{2}$ obtained in coincidence with $\mathrm{Si} 2 \mathrm{p}$ core electrons emitted only from stoichiometric $\mathrm{SiO}_{2}$, i.e. at $41.85 \pm 0.5 \mathrm{eV}$ kinetic energy $(\mathrm{h} \nu=150 \mathrm{eV})$. This coincidence Auger spectrum gener a.lly agrees with the calculation (dotted line) of Ramaker (Phys. Rev, B 21, 4608 (1980)). The disagreement near threshold is attributed to $\mathrm{Si}$ orbitals of d-character participating in the Auger transition and not, as previously suggested, to substoichiometric oxide generated by beam damage (the latter is explicitly eliminated by measuring in coincidence with only stoichiometric $\mathrm{SiO}_{2}$ ). R. A. Bartynski, A. K. See (Rutgers U.), C.-C. Kao, and S. L. Hulbert (NSLS). 


\section{(5) Time resolved photoemission}

Photoemission from laser-excited states has long been the exclusive domain of laser pump, laser probe techniques. For the first time, excited state photoemission has been demonstrated using a laser pump, synchrotron probe tech. nique at beamline X24C (see Fig. 30). The laser transiently popu- lates a surface state pocket at $\overline{\mathrm{X}}$ in the surface Brillouin zone of GaAs(110), which can be observed as a peak at a kinetic energy of $5.7 \mathrm{eV}$ using synchrotron radiation at $\mathrm{h} \nu=8.4 \mathrm{eV}$. The broad tunability of the synchrotron probe at these photon energies makes this technique useful for excited state photoemission in a wide range of materials. The $\mathrm{X} 24 \mathrm{C}$ researchers have also measured the time decay of laser-induced surface photo. voltage (SPV) shifts of the Si $2 p$ core level on Si(111) surfaces, and the elimination by the SPV of in. homogeneous broadening caused by band-bending nonuniformities on GaAs(110) surfaces (see Fig. 30).
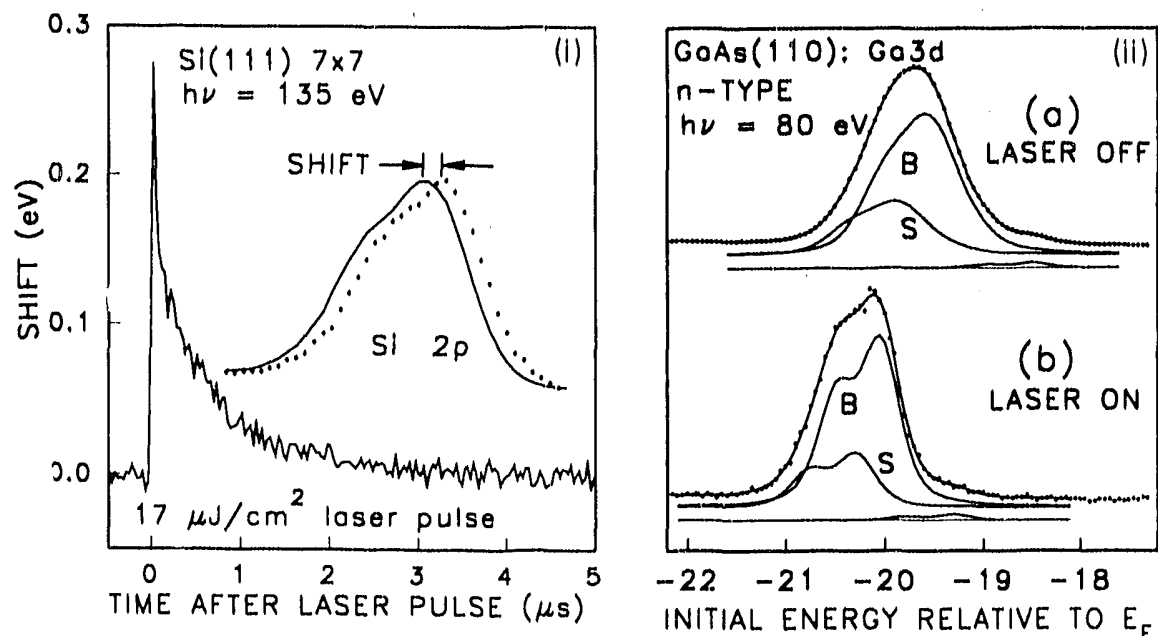

Fig. 30 Time resolved photoemission with nanosecond resolution, used to study laser excited states of semiconductors at beamline X24C. Much of these data are acquired during single bunch operations, which decreases by pver an order of magnitude the mismatch between laser and syn. chrotron pulse repetition rates. Clockwise from upper left: (i) Time decay of the laserinduced surface photovoltage (SPV) shift of the Si 2p core lovel. Analysis of such decay curves has characterized surface space-charge dynamics and has revealed extremely large surface recombination rates for a variety of $S i(111)$ surface preparations. (ii) Elimination by the SPV of inhomogeneous broadening caused by band-bending nonuniformities on GaAs(110). The narrowing of spectra recorded during the 5ns laser pulse aided in. terpretation of an unexpected surface photodissociation discovered in these experiments, (iii) Excited state photoemission. Until now, exclusively the domain of laser-pump-and-probe experiments, excited state photoemission was demonstrated for the first time using a synchrotron probe. The peak at $5.7 \mathrm{eV}$ orig. inates from electrons which transiently photo-populate a surface state pocket at $\bar{X}$ in the $\mathrm{GaAs}(110)$ surface Brillouin zone. J. P. Long, S. S. Goldenberg, J. C. Rife, and M. N. Kabler (NRL). 
EXAFS work carried out by PRT' and by general users is summa. rized in the abstracts of beamlines $\mathrm{X} 9 \mathrm{~A}, \mathrm{X} 11 \mathrm{~A}, \mathrm{X} 10 \mathrm{C}, \mathrm{X18B}, \mathrm{X} 19 \mathrm{~A}$, $\mathrm{X} 23 \mathrm{~A} 2$, and $\mathrm{X} 23 \mathrm{~B}$ found in the second volume of this Annual Report. The number of physical and life science programs making use of XAS techniques is ever increasing, and the beam time allocation process through the general user proposal system is now highly competitive.

One example of the importance of XAS in biological systems is the determination of the function of the E7 amino acid (histidine) in mammalian myoglobin. Researchers at X9 have reported the structure of the myoglobin oxygenbinding active site in which the $\mathrm{E} 7$ histidine is replaced by tyrosine. They conclude from EXAFS and optical absorption data that tyrosine binds cirectly to heme in its ferric form and does not allow any ligands to interact with the myoglobin heme.

Another example of the importance of the XAS technique is its application to environmental research. Soil contamination by toxic heavy metals is a major and grow. ing national environmental issue. DuPont Engineering researchers at X11 have used fluorescence EXAFS to determine that the lead in a typical environmental material, waste pond sludge containing $0.23 \% \mathrm{~Pb}$ by weight, is predominantly in the form of lead sulfide. A likely source of lead sulfide is the action of sulfate-reducing bacteria under anaerobic conditions. Lead sulfide is expected to present minimum hazard to the groundwater, but care needs to be taken that it is not converted to a soluble form by any other activity undertaken at the site.

ting studies to be performed on systems where no experimental standards are available, experience has shown that analysis using an FEFF calculated standard is more reliable than one using an experi. mental standard which is structur. ally or chemically too different from the unknown. Efforts are underway to extend FEFF to perform multiple scattering calculations and thereby accurately simulate spectra for atoms beyond the first coordination shell.

One of the new experimental tech. niques demonstrated at the NSLS during the past year overcomes one of the fundamental stumbling blocks for high resolution XANES spectroscopy: the broadening arising from the core hole lifetime. In standard XANES spectroscopy, the limited lifetime of the core hole produced in the photon absorption process results in an uncertainty in the energy of the final state and hence a smearing of the edge features in the absorption spectrum.

As a whole, the field of x-ray spectroscopy continues to mature as a result of the international effort to define standards for experimental practice and data analysis, and individual efforts aimed at developing accurate theories for simulating experimental spectra. The FEFF program, developed by John Rehr and coworkers at the University of Washington, is now in regular use as part of the data analysis procedure. The program computes theoretical EXAFS spectra from first principle for an arrangement of atoms with essentially a single adjustable parameter: an overall amplitude scaling factor arising from many-body effects which is uncertain to within about $20 \%$. In addition to permit-
This effect becomes more pro. nounced as the atomic number of the absorber increases. The new high-resolution XAS technique developed by $\mathrm{K}$. Hämäläinen, J. B. Hastings, D. P. Siddons, and L. E. Berman (NSLS) using X25 is based on high resolution analysis of the fluorescence radiation emitted when the core hole decays, as represented by the diagram in Fig. 31.

By counting only fluorescent photons which have energies within a narrow range, the energy of the initial core hole (and also, via energy conservation, the energy of the excited electron) is fixed. The longer lifetime of the secondary hole created in the fluorescence process results in a substantial reduction in 
Fig. 31 Schematic representation of high,resolution X-ray fluorescence spectroscopy as compared to standard (low res. olution) fluorescence measurements. In the high resolution fluorescence measurement, only a small energy slice of the emitted fluorescence radiation is accepted. By conservation of energy, this improves the energy resolution of the X-ray absorption measurement.

the overall energy broadening. The remarkable improvement in the structural detail evident in edge regions is represented in Fig. 32, which compares normal and highresolution XANES measurements for the Dysprosium L(III) edge.

The technique has also been used to perform spin-polarized measurements on anti-ferromagnetically coupled Mn samples.

Another experimental technique developed in the past year is diffraction anomalous fine structure spectroscopy (DAFS). In DAFS spectroscopy, developed by Charles Bouldin and coworkers at NIST and Larry Sorensen, J. J. Rehr and coworkers at the University of Washington (see abstracts for X23A2, and also independently $\mathrm{X} 10 \mathrm{C})$, the intensity modulations in a diffraction peak are measured as the incident energy is varied through and well past an absorption edge of one of the elements contributing to the structure factor. The experimental geometry, shown in Fig. 33, is similar to a conventional diffraction experiment, but with an energy-scanned incident beam.
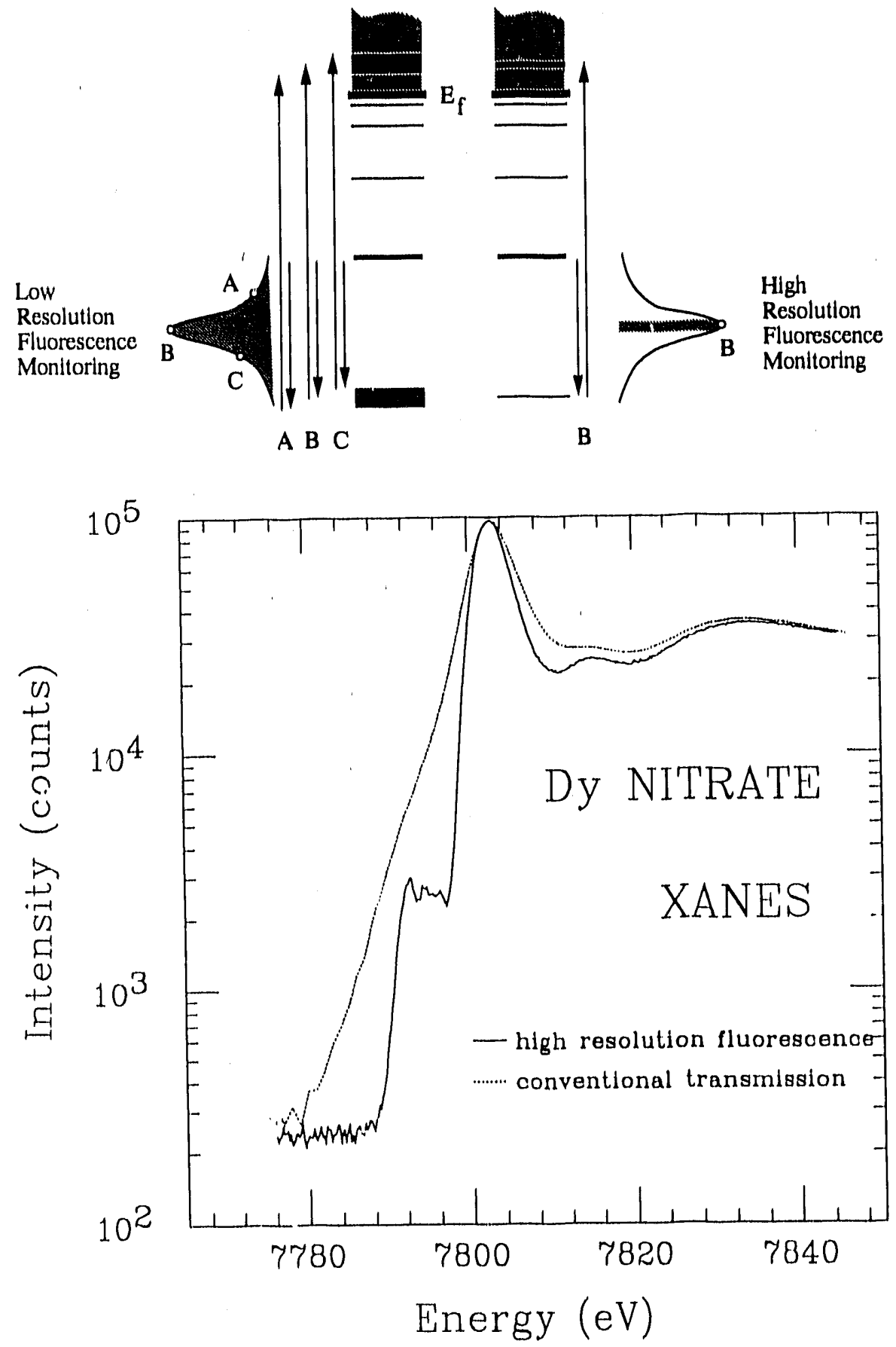

Fig. 32 The energy resolution of XAS has for the most part been limited by the natural lifetime broadening of the core hole. If, however, the energy of the fluorescent photons produced in the decay of this core hole can be determined with a resolution narrower than the lifetime width, the core hole lifetime broadening can be overcome. To realize this, a high resolution fluorescence spectrometer, based on a backscattering spherically-bent silicon crystal in a Johann geometry, was constructed and tested at X25. Shown above is the observed XANES spectrum of a $\mathrm{Dy}\left(\mathrm{NO}_{3}\right)_{3}$ sample at the $\mathrm{Dy} \mathrm{L}(\mathrm{III})$ edge, for which the analyzer window was centered on the Dy $L \alpha_{1}$ fluorescence line. A conventional transmission XANES spectrum of the same sample is shown for comparison. The enhanced resolution using the backscattering analyzer reveals fine structure which is totally invisible in the conventional spectrum. K. Hämäläinen, D. P. Siddons, J. B. Hastings, and L. E. Berman (NSLS); see Phys. Rev. Lett. 67, 2850 (1991). 
Fig. 33 The experimental setups for EXAFS /transmitted, or forward scattered signal) and DAFS $(\Theta, 2 \theta$ diffructed signal) spectroscopies.
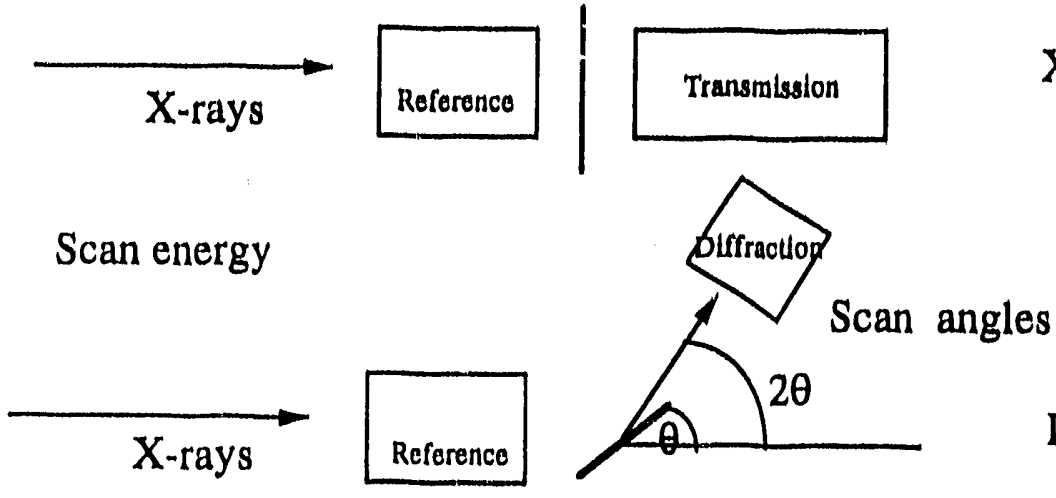

DAFS
The observed intensity variations in a 111 reflection from a copper crystal are shown in Fig. 34 (a), suporimposed upon the absorption spectrum.

The region after the edge is seen to contain oscillations analogous to EXAFS, and indeed the same Fourier transform magnitude (Fig. 34 (c)), is obtained after subtraction of a smooth background. But the oscillations are, in fact, $90^{\circ}$ out of phase (Fig. 34 (b)) with the EXAFS oscillations since the absorption is linked to scattering via a Kramers-Kronig transform.

The significance of DAFS arises from the fact that it generalizes EXAFS from an energy scanning spectroscopy to one that uses both photon momentum and photon en. ergy simultaneously. This double tunability retains the chemically selective character of EXAFS, while adding several important new capabilities. For instance, it is possible to use different diffraction peaks to study separately each phase of a multi-phase material. This has been demonstrated by a study of a multilayer system of $\mathrm{In}_{\mathrm{x}} \mathrm{Ga}_{1-\mathrm{x}} \mathrm{As}$ in which the structure of buried layers of different In composition (and hence, different lattice constants) was determined using DAFS measurements at three different photon momentum transfers, Q. In addition to macroscopic spatial selectivity, DAFS also extends the elemental selectivity of EXAFS to include site selec- tivity, i.e., by using DAFS spectra with different photon momentum transfers, it is possible to separately determine the short-range order about crystallographically inequivalent, but chemically equiv. alent atoms. This has been demonstrated by DAFS measurements of the two inequivalent sites of $\mathrm{Cu}$ atoms in the 123 high $\mathrm{T}_{\mathrm{c}}$ superconductors. The addition of siteselectivity to EXAFS has long been sought, but has never been satisfactorily achieved until the use of DAFS. Work on X10C shows that DAFS measurements can be made on powder samples, which will lead to applicability of this method to a very wide range of materials. 


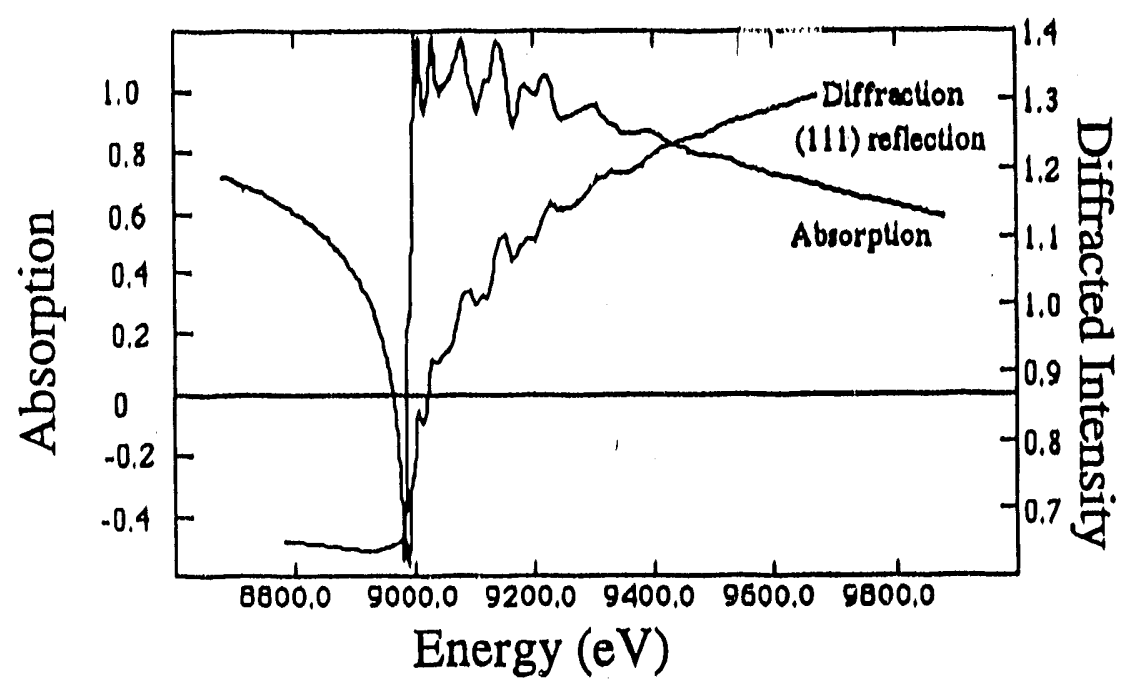

XAFS and DAFS Filtered First-shell Data
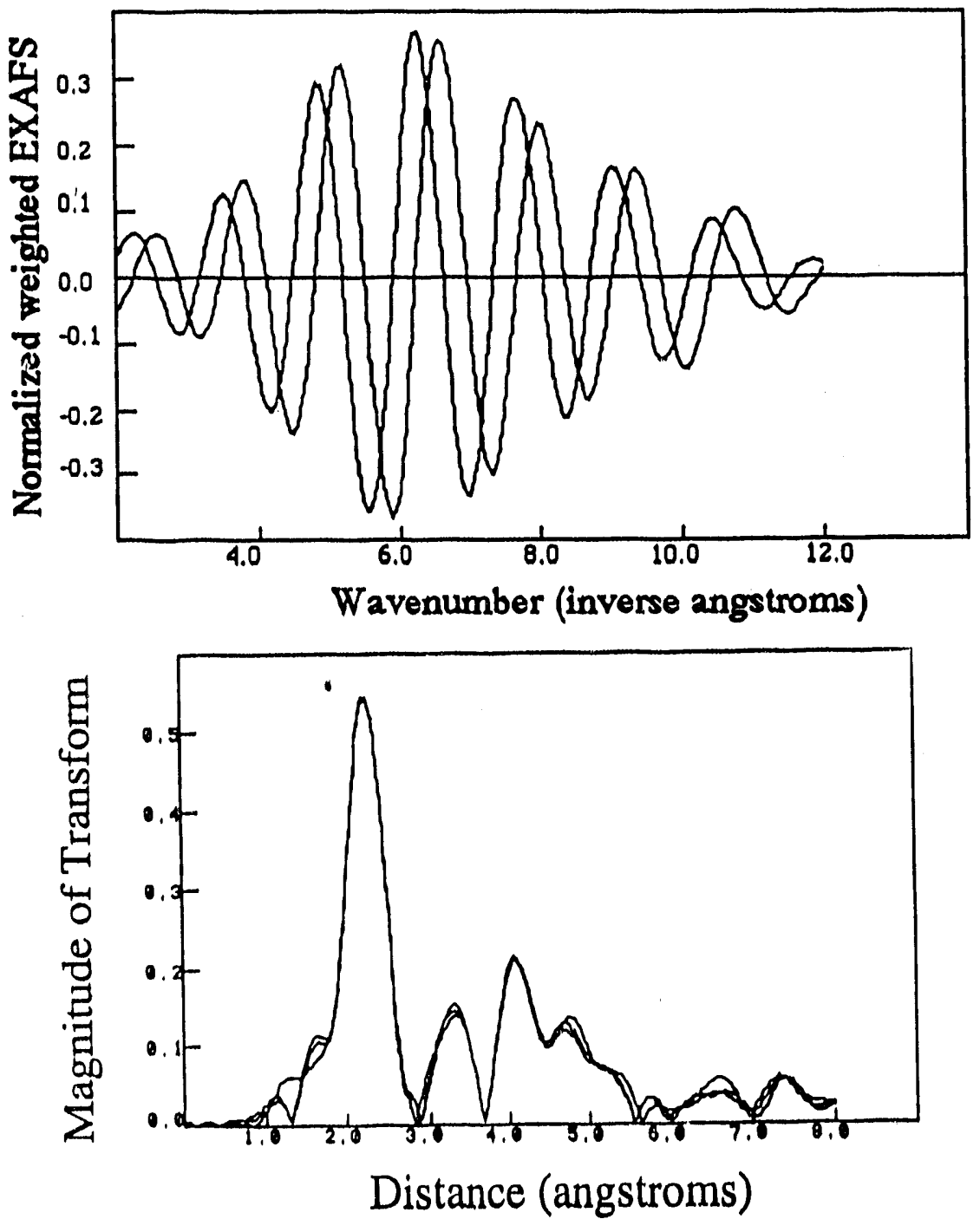

Fig. 34 Superposition of the EXAFS and DAFS spectra from a copper crystal: a) Raw data; b) Isolated first shell oscillations illustrating $\pi / 2$ phase shift; and c) Fourier transforms, J. O. Cross, H. Straigier, L. B. Sorensen (U. of Washington), C. E. Bouldin, and J. Wolcik (NIST). 


\section{X-RAY SCATTERING \\ AND CRYSTALLOGRAPHY}

\section{Lonny Berman \\ NSLS}

\section{Peter Siddons \\ NSLS}

X-ray scattering and crystallography research at the NSLS is performed on two-thirds of the X-ray Ring experimental stations, and comprises about half of the x-ray experimental abstracts in this Annual Report. Glancing incidence studies of surfaces and interfaces, magnetic scattering studies of anti-ferromagnetic and heavyFermion systems, powder diffraction studies of new complex materials such as Fullerenes and high- $\mathrm{T}_{\mathrm{c}}$ super-conductors, precession crystallography studies of protein structure, and small-angle scattering studies of polymers and other amorphous materials and liquids continued to predominate in FY91, while new scattering methods such as intensity fluctuation spectroscopy ("speckle" diffraction), diffraction anomalous fine structure (DAFS), and time resolved Laue-diffraction protein crystallography emerged.

In an experiment by $M$. Sutton et al. at X25, intensity fluctuation spectroscopy was carried out for the first time using hard x-rays. The method involves illuminating a sample with a coherent light beam. Different phase shifts are introduced into cornponents of the beam scattered by different re- gions of the sample, resulting in a speckled scattered beam image. By studying how the image changes with time, the time evolution of the arrangement of the scattering regions can be determined. In the X25 experiment, the sample was a $\mathrm{Cu}_{3} \mathrm{Au}$ crystal which had been quenched from the disordered to ordered phase. The scattered beam was the (100) Bragg peak, which is not allowed for the disordered phase, and the scatter- ing regions were the ordered antiphase domains. Intensity fluctuations were observed in a portion of the Bragg peak image, which arose from the growth of the domains following the quench (see Fig. 35).

The Diffraction Anomalous Fine Structure (DAFS) technique is analogous to Extended X-ray Absorption Fine Structure (EXAFS), in that short-range

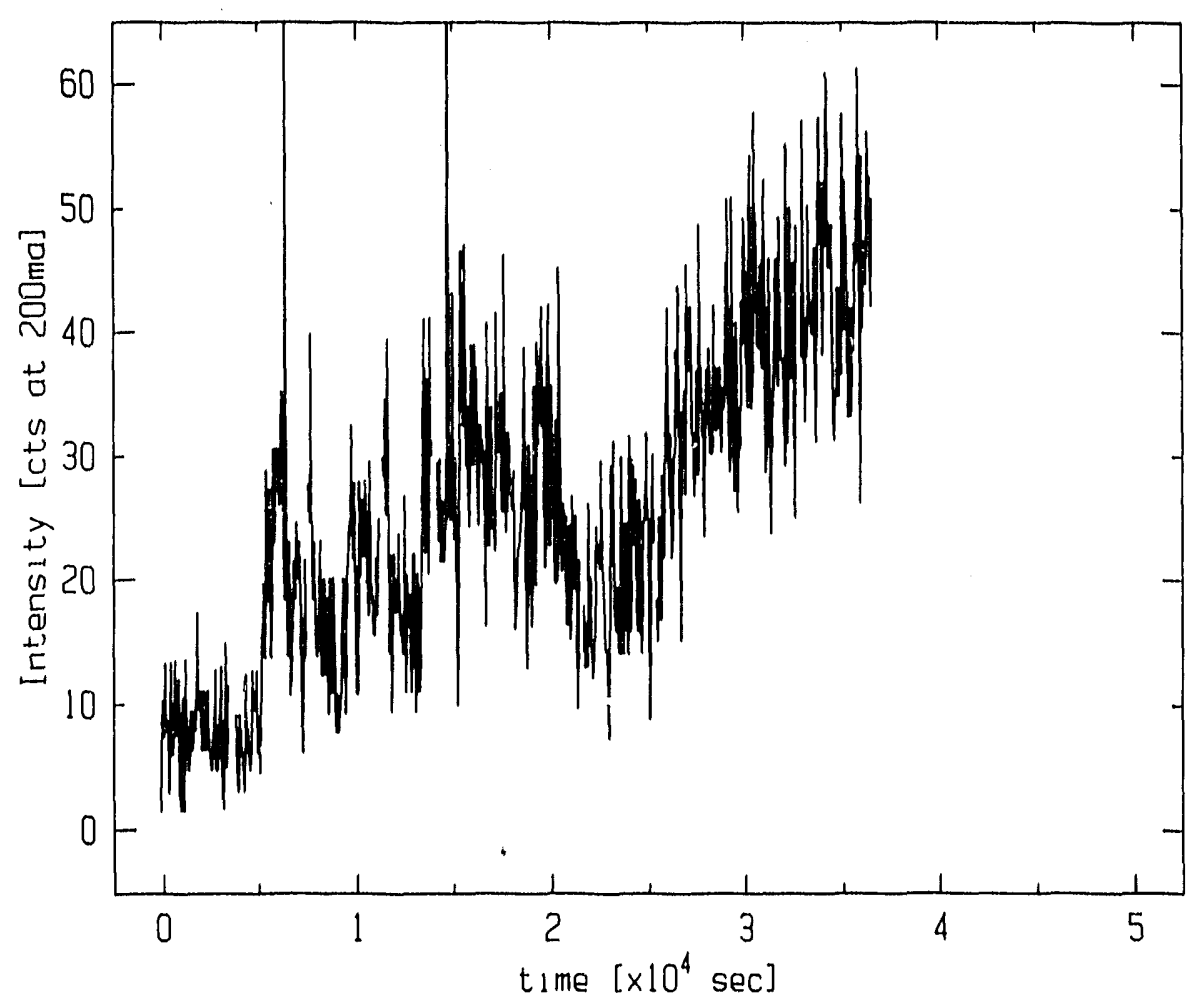

Fig. 35 When a coherent light beam illuminates a disordered sample, components of the beam scattered by different regions of the sample can have different phase shifts, introducing (in the far field) a speckled diffraction image that results from the constructive and destructive interference of the beam components. By observing how the image changes with time, it is possible to determine the time evolution of the arrangement of the scattering regions. Alternatively, the intensity variation of a single point of "speckle" in the image gives information on the time correlations of the sample inhomogeneities. This technique, called intensity fluctuation spectroscopy, was demonstrated for the first time with hard x-rays at $\mathrm{X} 25$. Shown above is the intensity of a single speckle in a $\mathrm{Cu}_{3} \mathrm{Au}(100) \mathrm{Bragg}$ peak using a coherent incident beam, following a rapid temperature quench from the disordered phase (for which the (100) peak is not allowed) to the ordered phase at time zero. The time evolution of the growth of ordered antiphase domains gives rise to the observed intensity fluctuations. $\mathrm{Had}$ an incoherent incident beam been used, a purely monotonic increase in intensity (with. out any fluctuations) would have resulted. M. Sutton, E. Dufresne (McGill U.), S. G. J. Mochrie (MIT), L. E. Berman (NSLS), G. Held, and G. B. Stephenson (IBM). 
structural information can be extracted by Fourier-transforming an anomalous-scattering dependent signal, recorded as a function of photon energy above an absorption edge. In the case of EXAFS, the signal of interest results from $\mathrm{x}$-ray absorption, and is directly related to the imaginary part of the anomalous scattering amplitude. In the case of DAFS, the signal of interest is a Bragg peak intensity, which contains contributions from both the real and imaginary parts of the scattering amplitude. Experimesis by Bouldin et al. at X23A2 and Pickering et al. at X10C illustrated how DAFS can be used to obtain site-selective short-range structural information that is hard to come by using EXAFS. (See also $\mathrm{X}$-ray Absorption Spectroscopy in this section.)
Interest in Laue-diffraction crystallography has revived. In this technique, the continuurn of $x$-ray wavelengths from the source, rather than a single wavelength, is made available for use. The sample selects unique wavelengths to diffract, based on its structure and orientation, and the pattern is recorded on film or an image plate. In principle, only a few short exposures are required to obtain enough information to determine the crystal structure, since all of the $x$-ray intensity radiated by the source is used, and each exposure can sample a large portion of reciprocal space. The technique is ideal for studies of dynamic processes in large unit cell samples, such as protein crystals, for which an enormous number of reflections are required to determine the structure. The short exposure times can also allow full data sets to be recorded before the sample suffers from radiation damage. Prototype Laue diffraction measurements of the response of a crystallized enzyme, trypsin, to a $\mathrm{pH}$ jump, performed by Singer et al. at X25 and X26C, revealed subtle structural changes. Typical exposure times varied from 25 $\mathrm{msec}$ at X25 to $800 \mathrm{msec}$ at X26C.

Surface and interface reflectivity and diffraction studies have been especially popular at the NSLS. For example, Gibbs et al. at X22C have used high resolution surface $x$-ray diffraction to study details of the temperature-induced surface reconstructions of $\mathrm{Pt}(001)$ and $\mathrm{Au}(001)$ (see Fig. 36).

Complementary truncation rod reflectivity (Sandy et al., X20A)
Fig. 36 Orientational Epitaxy of the Hexagonally Reconstructed Au(001) and Pt(001) Surfaces: $\mathrm{X} 22 \mathrm{C}$ researchers have carried out extensive surface $\mathrm{x}$-ray diffraction studies of the structure and phase behavior of the low-index surfaces of $P t$ and $A u$. For the (001) surfaces, they have found a remarkable similarity in their phase behaviors when considered on a temperature scale normalized by their respective bulk meliting temperatures, $T_{m}$. Between $0.9 \mathrm{~T}_{\mathrm{m}}$ and $0.8 \mathrm{~T}_{\mathrm{m}}$, these surfaces display ordered, hexagonal structures which are incommensurate with bulk lattice planes of square symmetry lying immediately beneath, as evidenced by surface $x$-ray diffraction peaks of hexagonal symmetry. Below about $0.8 \mathrm{~T}_{\mathrm{m}}$, the hexagonally reconstructed domains rotate to positive and negative angles with respect to the substrate orientation. The temperature dependence of the rotation angle is shown in this figure, where the different symbols represent different coexisting rotated domains. In $\mathrm{Pt}$, the rotational transformation is continuous and exhibits mean-field behavior, while in $\mathrm{Au}$, it is discontinuous. These latter results are inconsistent with the predictions of current theories and motivate renewed consideration of orientational epitaxy, especially for metals. D. Gibbs, G. Grübel (BNL Physics Dept.), D. M. Zehner (ORNL), D. L. Abernathy, and S. G. J. Mochrie (MIT). See Phys. Rev. Lett. 67, 3117 (1991).

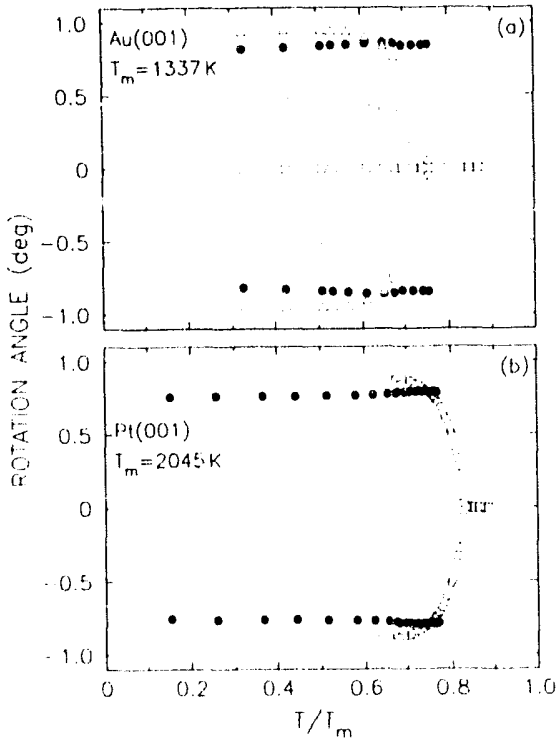


and surface diffraction (Grübel et al., X22C) measurements of the high-temperature reconstruction of the $\mathrm{Pt}(111)$ surface point to a compressed incommensurate surface layer consisting of regions of ideal and faulted stacking (see Fig. 37).

Surface diffraction measurements of the structure of $\mathrm{K}$ monolayers on a $\mathrm{Cu}(100)$ surface by Meyerheim et al. at X16A revealed a continuous change in the incommensurability of the overlayer with time following the initial deposition, which may have been caused by desorption, clustering, or contamination. Diffraction measurements of the $\mathrm{Ag} / \mathrm{Si}$ (111) buried interface by Hong et al. at X14A confirmed the preservation of the initial $\mathrm{Si}(111) 7 \times 7$ reconstruction following room temperature deposition of a thick Ag film; the reconstruction disappeared after annealing the interface. In experiments by Tidswell et al. at X16B, diffraction and reflectivity were used to learn about the structure and roughness of both the liquid-solid and liquidvapor interfaces for a hydrocarbon film on a Si wafer. Electrochemical electrode surfaces were studied with $\mathrm{x}$-ray diffraction and reflectivity by You et al. at X10B, Toney et al. and Wiesler et al. at $\mathrm{X} 20 \mathrm{~A}$, Armstrong et al. at X20B, and 0 cko et al. at X22B/X25 (see Fig. 38).

Real-time studies of film growth on surfaces have also been performed using $x$-ray diffraction: Au growth on $\mathrm{Si}$ was studied by Chiarello et al. at X6B and X22B, $\mathrm{Pd}_{2} \mathrm{Si}$ growth on $\mathrm{Si}(111)$ was examined by Bennett et al. at X16A,

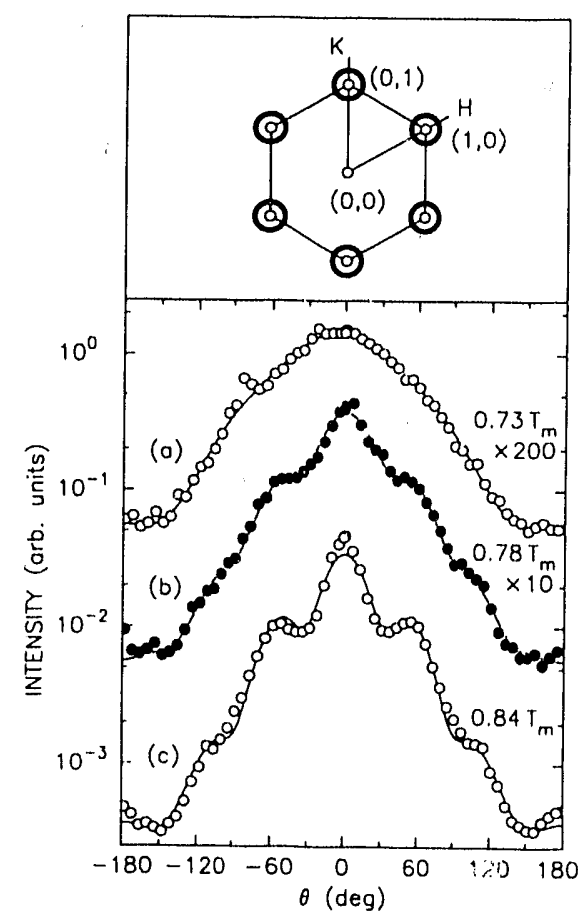

Fig. 37 The structure of the clean Pt(111) surface has been studied between $300 \mathrm{~K}$ and $0.9 \mathrm{~T}_{\mathrm{m}}\left(\mathrm{T}_{\mathrm{m}}=2045 \mathrm{~K}\right)$ via $\mathrm{x}$-ray scattering at beamline X22C. The surface is unreconstructed for temperatures less than $0.65 \mathrm{~T}_{\mathrm{m}}$, but reconstructs at higher temperatures to form a layer isotropically compressed and incommensurate with the underlying bulk (111)-planes. A disordered arrangement of discommensurations separates regions with ideal fcc stacking from regions with faulted stacking. With increasing temperature, both the compression of the surface layer and the orientational order of the discommensurations increase. Upper figure: the diffraction pattern obtained for $\mathrm{Pt}(111)$ at high temperatures in the reconstructed phase. Lower figure: "ring scans" taken around the $(1,0)$ reflection as a function of temperature. The scattering function shows a clear six-fold angular moduation, the amplitude of which increases with increasing temperature. A. R. Sandy, S. G. J. Mochrie (MIT), D. M. Zehner (ORNL), G. Grübel, K. G. Huang, and D. Gibbs (BNL Physics Dept).

and Xe growth on $\mathrm{Ag}(111)$ was studied by Wang et al. at X18A. Anomalous reflectivity methods were employed to learn about film stucture by Bai et al. at X6B and Sanyal et al. at X22C. The X-ray standing wave method was used to study alkali overlayer registry on $\mathrm{Si}(111)$ by Lagomarsino et al. at $X 15 \mathrm{~A}$, high- $\mathrm{T}_{\mathrm{c}}$ film quacity by Zegenhagen et al. at X15A, rare gas adsorption on a graphite single crystal by Swanson et al. at $\mathrm{X} 24 \mathrm{~A}$, and the $\operatorname{InP}(110)$ surface reconstruction by Woicik et al. at X24A.

Thermal diffuse x-ray scattering continued to be employed for strain and precipitation studies in alloys and compounds, by Butler et al. and Larson et al. at X14A,

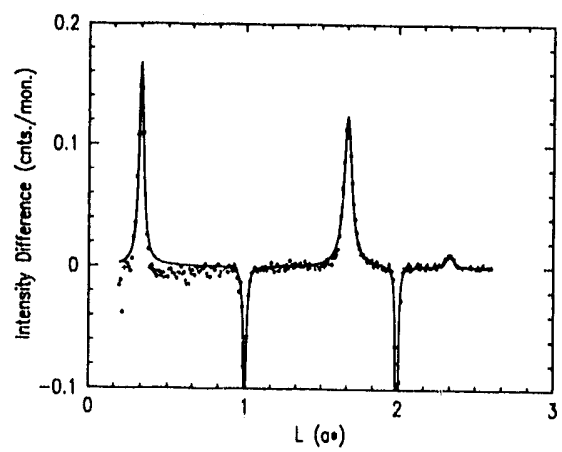

Fig. 38 Potential Induced $(1 \times 3)$ Reconstruction of the $A u(110)$ Surface: Whereas the clean $\mathrm{Au}(110)$ surface exhibits at $(1 \times 2)$ reconstruction in UHV, at an electrochemical interface we observe a $(1 \times 3)$ structure. X-ray scattering studies of the Au(110) electrode surface have been carried out at $\mathrm{X} 22 \mathrm{~B}$ and $\mathrm{X} 25$ versus the applied potential in $0.1 \mathrm{M} \mathrm{NaF}, \mathrm{NaCl}, \mathrm{NaBr}, \mathrm{LiCl}$, and $\mathrm{CsCl}$ solutions under potential control. At sufficiently negative potentials, in these salt solutions, the surface forms a $(1 \times 3)$ reconstruction with (111) microfacets. Above a critical threshold potential, the $(1 \times 3) \mathrm{re}$ construction vanishes and the surface forms a $(1 \times 1)$ structure. The threshold potential depends on the electrolyte, and in all solutions the transition between these two structures is completely reversible. This figure shows the intensity difference between scans along $(0.1,0.1, \mathrm{~L})$ at $-0.3 \mathrm{~V}$ and $0.0 \mathrm{~V}$ applied potentials in $0.1 \mathrm{M} \mathrm{NaCl}$. Diffraction peaks at $L=1 / 3,5 / 3$, and $7 / 3$ are consistent with the $(1 \times 3)$ missing-row model. B. M. Ocko, G. Helgesen, B. Schardt, J. Wang (BNL Physics Dept.), and $\mathrm{A}$. Hamelin (CNRS). 
and Mahadev et al. and $\mathrm{Na}$ et al. at $\mathrm{X} 18 \mathrm{~A}$. A recent application was for short-iange order and correlation determinations in liquid polymers, by Zhao et al. at X22B and $\mathrm{X} 22 \mathrm{C}$.

Magnetic x-ray scattering has been gaining more interest at the NSLS. Highlighting this field was the observation of critical magnetic scattering from the spiral antiferromagnet $\mathrm{Ho}$ in the vicinity of its Néel temperature, by Thurston et al. at X22C (see Fig. 39).

$\mathrm{C}_{60} \quad$ Buckminster-Fullerenes ("Bucky-balls") are of great interest at the moment, and some interesting powder diffraction studies have been performed at the NSLS. Heiney and co-workers studied orientational ordering phase transitions in $\mathrm{C}_{60}$ using the high-resolution capabilities of $\mathrm{X} 7 \mathrm{~A}$. The high resolution and good signal-to-noise allowed detailed intensity measurements to high orders, enabling the identification of two phases, one in which the $\mathrm{C}_{60}$ molecules had random orientation within the lattice, and another below $250 \mathrm{~K}$ whereby the molecules were ordered in sets such that they were rotated about local $\langle 111\rangle$ axes by about $25^{\circ}$ (see Fig. 40).

Another Bucky-ball experiment made use of the powder diffraction capability of X3A2. There, Stephens and his colleagues studied the structure of Fullerenes which were doped with alkali metals. They were able to show that in the $\mathrm{K}$-doped $\mathrm{C}_{60}$ (which is known to superconduct), the $K$ ions fit into interstitial sites, where they constrain the orienta-

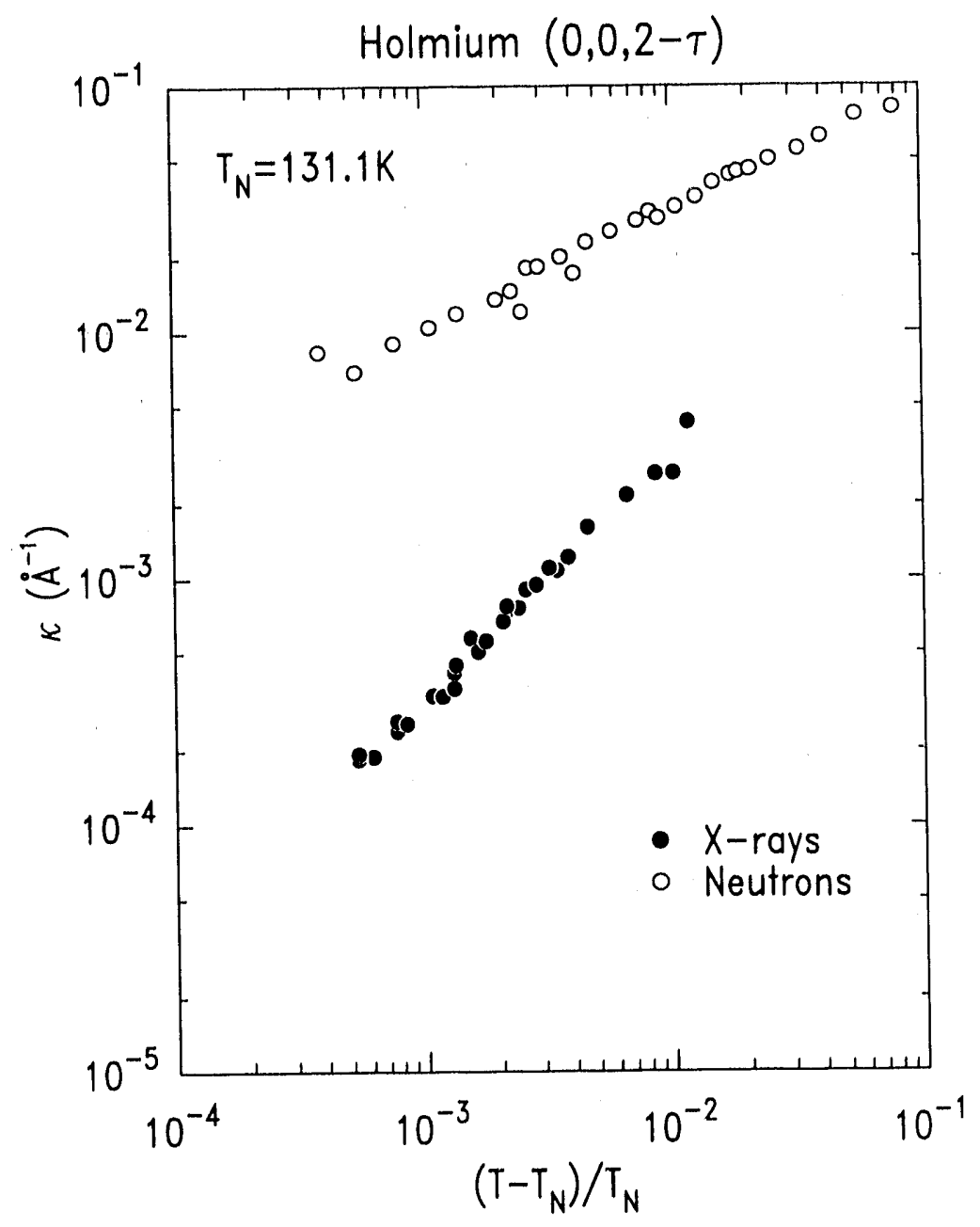

Fig. 39 Antiferromagnet Holmium Measurements: Comparison between the inverse correlation length in the spiral antiferromagnet holmium measured by $\mathrm{x}$-rays at beamline X22C and neutrons at BNL's High Flux Beam Reactor. Within a mescoscopic distance of the surface comparable to the $x$-ray penetration depth $(\sim 0.3 \mu \mathrm{m})$, the magnetism measured by $x$ rays is drastically different from the bulk magnetic behavior measured by neutrons. That the surface of a sample can affect antiferromagnetism over mesoscopic length scales is a surprising result not anticipated theoretically. T. 'Thurston, D. Gibbs, G. Shirane (BNL Physics Dept.), J. P. Hill (MIT), and B. Gaulin (McMaster U.).

tion of the $\mathrm{C}_{60}$ into the two possible states having eight hexagonal rings facing $<111\rangle$ directions. In contrast to the pure $\mathrm{C}_{60}$ discussed above, rotational disorder was shown to persist at least down to $11 \mathrm{~K}$. Other dopants were also studied (see Fig. 41).

A recent example of "surface powder diffraction" was carried out by Heald and Jayanetti on X11A. They were able to follow the crystallization of a $2000 \AA$ film of Ge covered with $1000 \AA$ of $\mathrm{Al}$. The presence of a metal layer is known to reduce substantially the crystallization temperature of amorphous $\mathrm{Si}$ and $\mathrm{Ge}$. Glancingangle powder diffraction, reflectivity, and EXAFS allowed this recrystallization process to be followed in a time-resolved manner, giving insight into the crystal nucleation and growth mechanisms (see Fig. 42). 
Fig. 40 Molecular structure of Buckminsterfullerene, $\mathrm{C}_{60}$. High resolution powder diffraction experiments carried out in a I'niversity of Pennsylvania - Brookhaven collaboration at beamline X7A have revealed the existence of an orientational ordering transition in $\mathrm{C}_{60}$ at $250 \mathrm{~K}$ and an analogous transition in $\mathrm{C}_{70}$. P. A. Heiney, J. E. Fischer, A. R. McGhie, W. J. Romanow, A. N. Denenstein, J. P. McCauley Jr., A. B. Smith (U. Penn.), and D. E. Cox (BNL Physics Dept.).

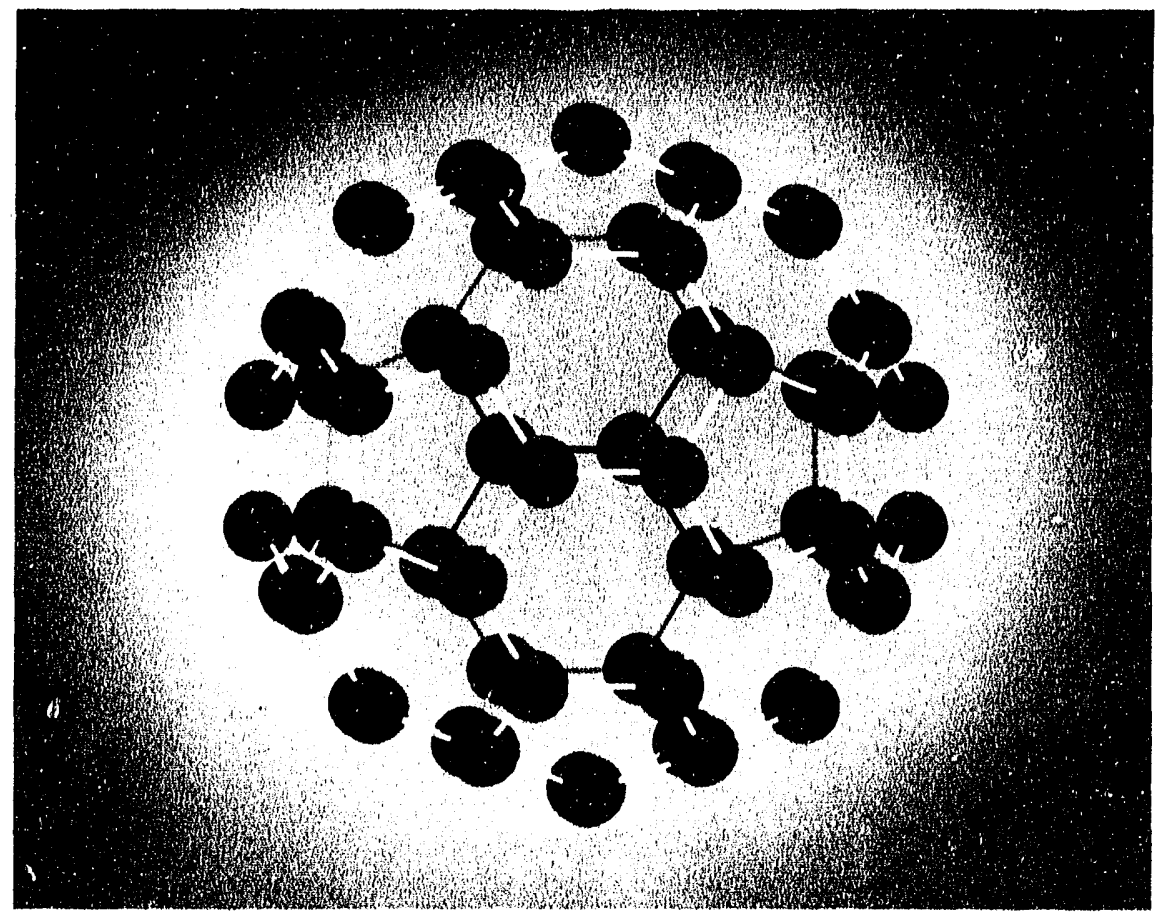

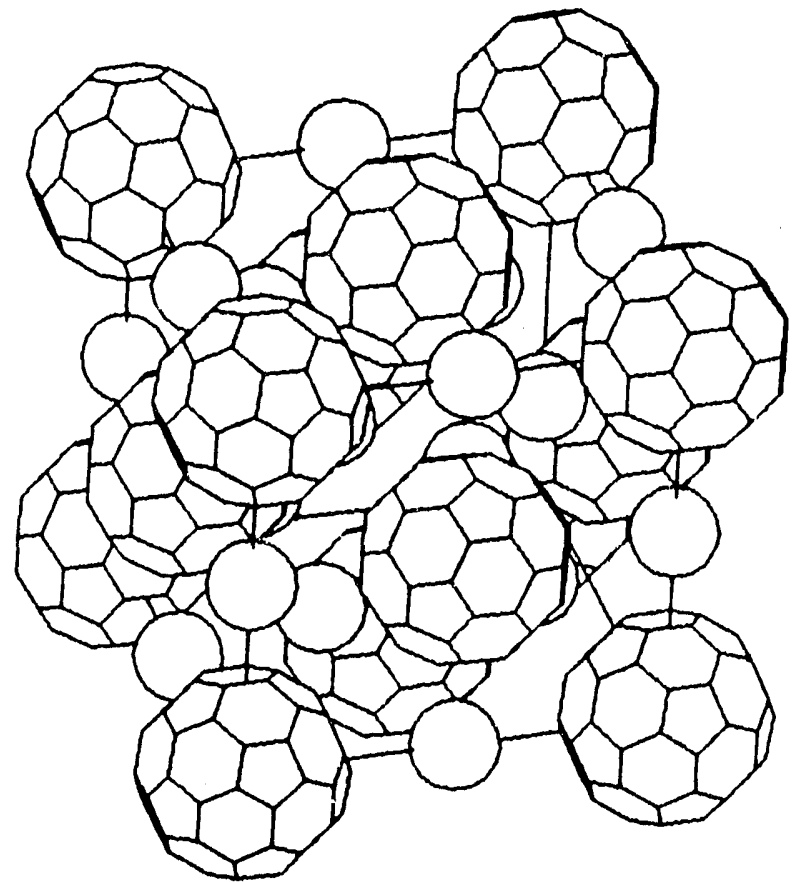

Fig. 41 The crystal structure of the superconducting Buckminster-fullerene compound, $\mathrm{K}_{3} \mathrm{C}_{60}$. The raspberries are $\mathrm{C}_{60}$, and the smaller isolated spheres are potassium cations, with different colors (see cover) in different crystallographic sites. This structure was solved by Peter Stephens and coworkers at SUNY and UCLA, from powder diffraction data taken at beamline X3. P. W. Stephens, L. Mihaly (SUNY at Stony Brook), F. Diederich, K. Holczer, S.-M. Huang, R. B. Kaner, R. L. Whetten, J. B. Wiley (UCLA), and P. L. Lee (SUNY at Buffalo). See Nature, 351, 632 (1991).
Synchrotron radiation is often quoted as being especially beneficial when samples are small and contained in difficult environ. ments. A good example of this type of application is the highpressure, high-resolution experiments performed by Jephcoat and coworkers using X7A. The combination of a horizontally. focussing monochromator and a linear position-sensitive detector resulted in moderately high flux together with efficient detection. The study illustrated, among other things, the crucial importance of the use of a pressuretransmitting fluid (in this case helium) in the reduction of strain gradients in the sample, and hence the ability to perform meaningful high-resolution measurements (see Fig. 43). 

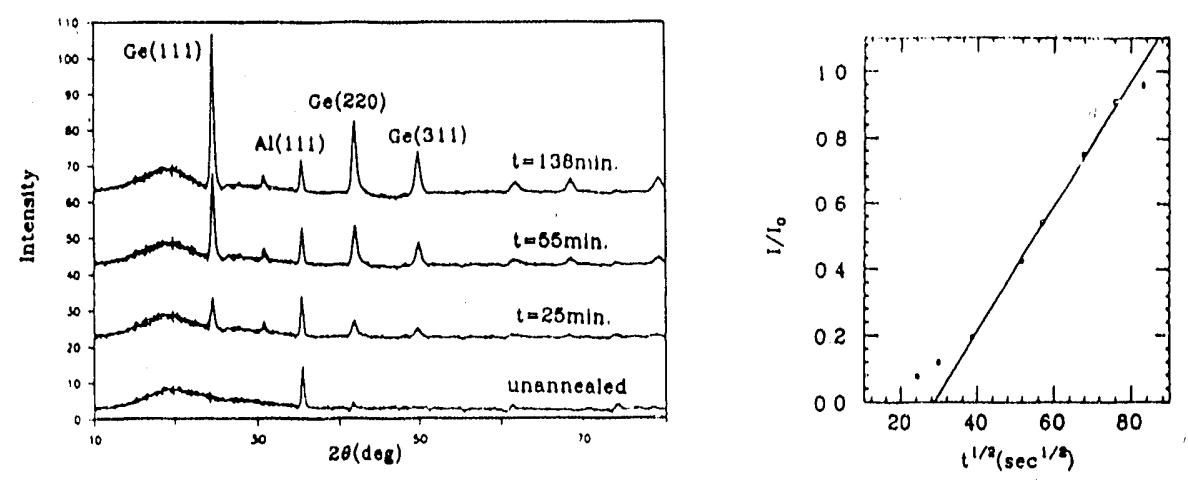

Fig. $42 \mathrm{X}$-ray diffraction data obtained at beamline X11A,B from a $2000 \AA$ amorphous Ge thin film covered with $1000 \AA$ of $\mathrm{Al}$, while annealing at $152^{\circ} \mathrm{C}$. On the left is the raw diffraction data at time zero and three annealing times. On the right is the intensity of the $\mathrm{Ge}(111)$ reflection plotted versus the square root of the annealing time. After an initial nucleation period the Ge diffraction intensity follows a $t^{1 / 2}$ time dependence. This indicates that after the reaction moves away from the interface it is diffusion limited, likely due to the need for the metal atoms to diffuse to the crystallization front. The enhanced diffusion caused by interaction of the semi zonductor $(\mathrm{Ge})$ and the metal (Al) substantially reduces the amorphous Ge crystallization temperature. S. M. Heald and J. K. D. Jayanetti (BNL Dept. of Applied Science).

Another often-quoted advantage of synchrotron radiation is its tunability, and the potential to use anomalous scattering as a way to record complex diffraction patterns. An interesting application of this idea was pursued by Warner et al. at X7A. Their aim was to try to distinguish the scattering within a molecule by atoms of the same element but with different valences. As is well-known, the absolute position of $\mathrm{x}$-ray absorption edges is sensitive to the chemical state of the absorbing species. Thus, very close to the nominal edge, different valence ions will have different anomalous scattering factors. Warner and his colleagues were able to demonstrate that scattering factor differences of from one to two electrons between $\mathrm{Fe}^{\mathrm{II}}$ and $\mathrm{Fe}^{\mathrm{III}}$ in $\alpha-\mathrm{Fe}_{2} \mathrm{PO}_{5}$ were observable.

As in other areas, small-angle $\mathrm{x}$ ray scattering with synchrotron radiation has concentrated on two aspects, the ability to make timeresolved measurements and to obtain high resolution. Examples of time-resolved measurements are the experiments on phase separation and crystallization kinetics in polymers performed on $\mathrm{X} 3 \mathrm{~A} 2$ by $\mathrm{Chu}$ and his colleagues. In addition to small-angle scattering, wide-angle diffraction can be performed simultaneously on this tions of the behaviors in both microscopic and macroscopic scale lengths. Such studies can lead to better understanding of these industrially-important materials and processes. In a similar vein, the experiments by Shen and collaborators at $\mathrm{X} 12 \mathrm{~B}$ to determine the phase diagram of a microem. ulsion involving a surfactant, decane, and water using small-angle $\mathrm{x}$-ray scattering showed its power to unravel a complex and untrack. able problem typical of those found in industrial processes. instrument, which allows correla-
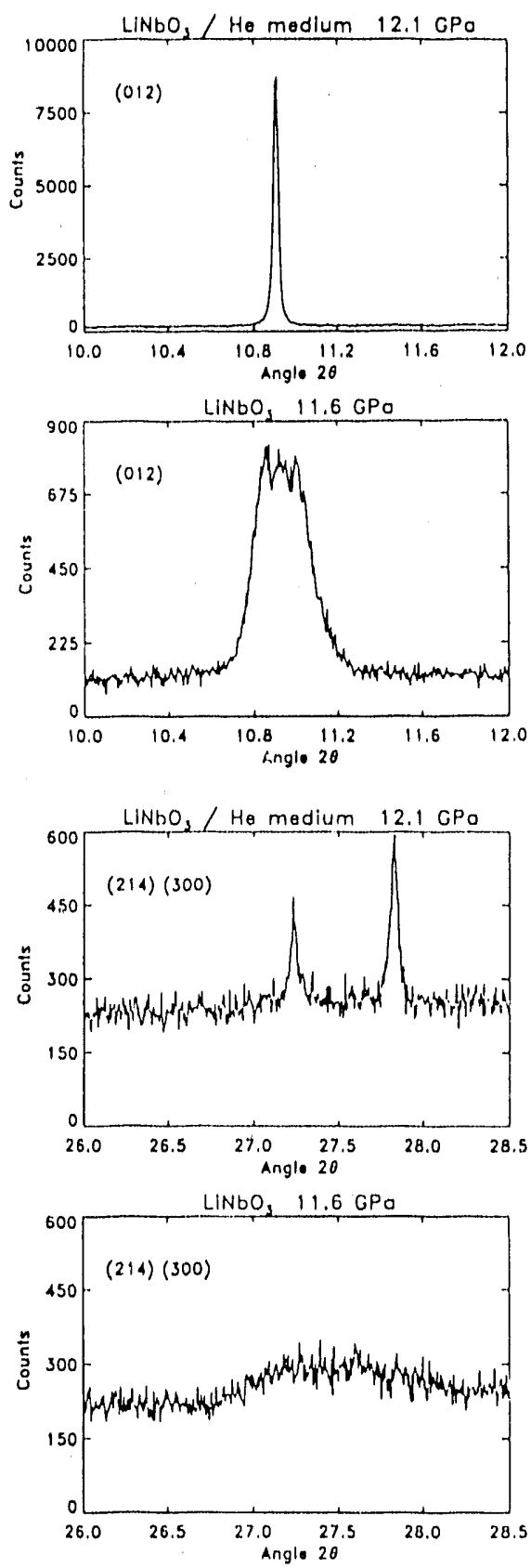

Fig, 43 High resolution, high pressure power diffraction results for ferroelectric $\mathrm{LiNbO}_{3}$ at $12 \mathrm{GPa}$ from beamline X7A The importance of a pressure medium is shown by the order of magnitude increase in line width obtained without solid helium as a medium. Use of a smaller collimator $(0.020 \mathrm{~mm}$ pinhole) does not result in narrower peak profiles, indicating that pressure gradients are not the dominant factor in lineshape at this pressure. A. P. Jephcoat (Oxford U.), L. W. Finger (CIW), and D. E. Cox (BNL Physics Dept.). 
Instruments such as those at $\mathrm{X} 3 \mathrm{~A} 2$ and $\mathrm{X} 12 \mathrm{~B}$ routinely achieve resolutions down to $q$-values of $10^{-2} \AA^{-1}$. There is a region below this which is inaccessible to such instruments. The device at X23A3 is designed to access the region $10^{-5}-10^{-1} \AA^{-1}$ using crystal collimators (the Bonse-Hart geometry) (see Fig. 44).

The studies of microporous foams by Olivier et al. is an example of an application of the instrument to learn about correlations in the length range normally considered the realm of visible light scattering. Of course, $x$-rays have the big advantage that they can address non-transparent samples, which makes the high resolution smallangle $x$-ray scattering technique much more generally applicable.

It is clear that the wealth of interest evident in x-ray scattering and crystallographic studies at the NSLS will ensure their continued predomination among the materials characterization tools in the future.

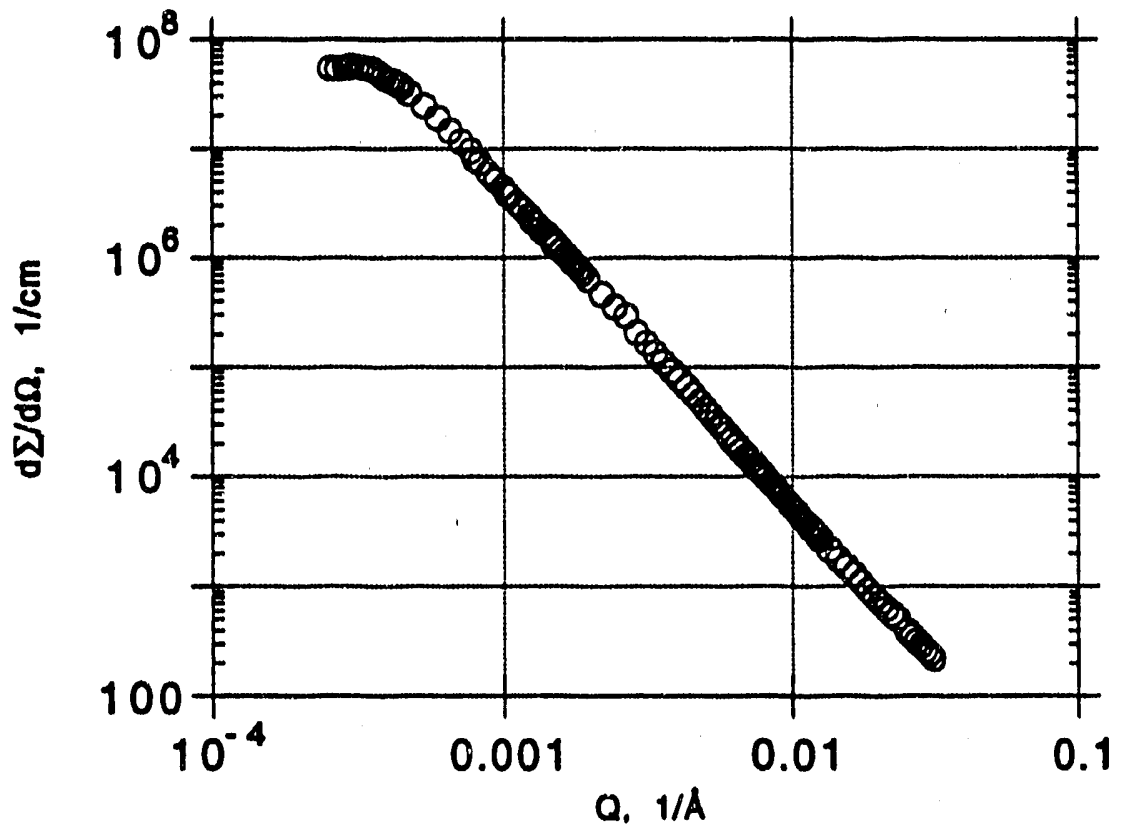

Fig. 44 Small Angle X-ray scattering (SAX) spectrum from beamline X23A3, plotted on a log-log scale, of a micropornus foam. B. J. Olivier, D. W. Schaefer (Sandia Nat. Lab.), S. Krueger and G. Long (NIST). 
X-RAY

\section{TOPOGRAPHY}

\section{Michael Dudley \\ SUNY at Stony Brook \\ Special Interest Group \\ Representative}

M. Dudley and his group, in collaboration with $\mathrm{D}$. Paine from Brown University and R. N. Sacks from United Technologies Research Center, have applied the technique of topographic imaging in grazing Bragg-Laue geometries, recently developed on X19C, to the analysis of defects as a function of depth in a $200 \mathrm{~nm}$ thick $\operatorname{In}_{\mathrm{x}} \mathrm{Ga}_{1-\mathrm{x}}$. As strained layer system, achieving a depth resolution better than $10 \mathrm{~nm}$. This level of resolution is unprecedented in topography.

On beamline X23A3 David Black and Harold Burdette studied the microstructure of natural and man-made diamond. The relationship between growth conditions and defect microstructure of man. made diamond was investigated. It was found that the quality of the grown crystals varies dramatically from the seed end to the top of the crystal. The quality of type Ia and II natural diamond is being studied to determine the best quality diamond for use as substrates for homoepitaxial growth of CVD diamond films. The relationship between the microstructure of these natural substrates and the quality of the CVD films is being studied.
H. H. Schloessin, R. D. Spal, and R. A. Secco have used the hard $x$-ray microscope developed at $\mathrm{X} 23 \mathrm{~A} 3$ to investigate pressure or percussion figures in single crystals of calcite. These figures, which characterize the cohesive strength of the crystal as dependent upon structure, are created by dynamic loading which was applied to the crystal with a newly developed indentor mounted to the microscope. A complete set of diffraction images was obtained for one crystal from the point of touch down of the indentor to fracture. These images showed that the pressure figure consists of a twinned lattice.

M. Dudley and his group on beamline X19C, in collaboration with Julia Phillips' group of AT\&T Bell Labs, have made considerable progress in their project relating to the non-destructive characterization of crystals which are candidates for use as substrates for high $\mathrm{T}_{\mathrm{c}}$ superconductor epilayers. Twin operations in $\mathrm{LaAlO}_{3}$ single crystals were solved. In addition the second order phase transition at $435^{\circ} \mathrm{C}$ was studied in situ (see Figs. 45 and 46).

M. Dudley and his group on beamline X19C, in collaboration with Bruce Foxman of Brandeis University and William Jones of Cambridge University, have made significant progress in the characterization of defects in $p$. terphenyl single crystals. Complete dislocation and twin analsis has been performed. Following this, the low temperature monoclinic to triclinic phase transition at $130^{\circ} \mathrm{K}$ was studied in situ.

M. Dudley and his group on beamline X19C, in collaboration with D. Hodul of Varian Research center, made observations of dis. location motion in high carbon content $\mathrm{Si}$ induced by rapid thermal processing. Nucleation of dislocations at precipitates was observed, followed by combined glide and climb.

M. Dudley and his group on beamline X19C, in collaboration with the group of I. Baker at Dartmouth, have continued to make significant progress on their project concerning the examination of ice single and polycrystals. In situ deformation experiments have been performed on bicrystals which shed much light on deformation mechanisms in polycrystalline ice. In particular the nucleation of dislocations at grain boundaries was observed.

M. Dudley and his group on beamline X19C, in collaboration with David Larson of Grumman Aerospace Corporation, have made significant breakthroughs in their project concerning the characterization of growth defects in CdZnTe single crystals. A new method for the characterization of twin operations has been developed. Similarly, progress has been made in the characturization of MBE grown $\mathrm{CdTe}$ in collaboration with Bob Silberstein, Myung Lee and Don DiMarzio of Grumman. 
M. Dudley and his group on beamline X19C, in collaboration with C. Fazi of U.S. Army Harry Diamond Laboratories, and D. Gordon-Smith of University of Warwick, continue to make significant progress in their studies of the distribution of breakdown damage in epitaxial silicon $p-n$ junctions. This has enabled signif- icant contributions to be made to the understanding of the fundamentals of the breakdown process for the cases of forward and reverse bias, as well as for combinations of these.

M. Dudley and his group on beamline X19C have made progress in a new project involving high temperature deformation studies on InSb. Dislocation configurations around high tempera. ture indentations afford much information on the relative mobilities of screw and $60^{\circ}$ dislocations in this material.
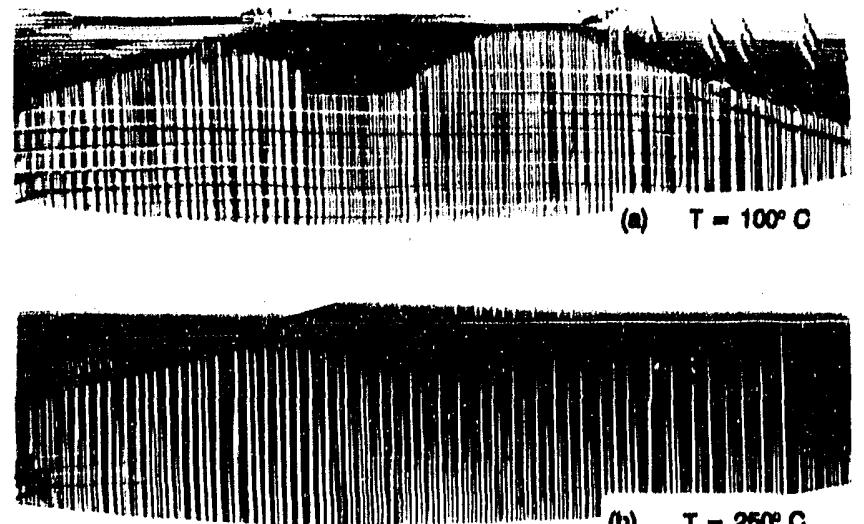

(b) $\mathrm{T}=2200^{\circ} \mathrm{C}$
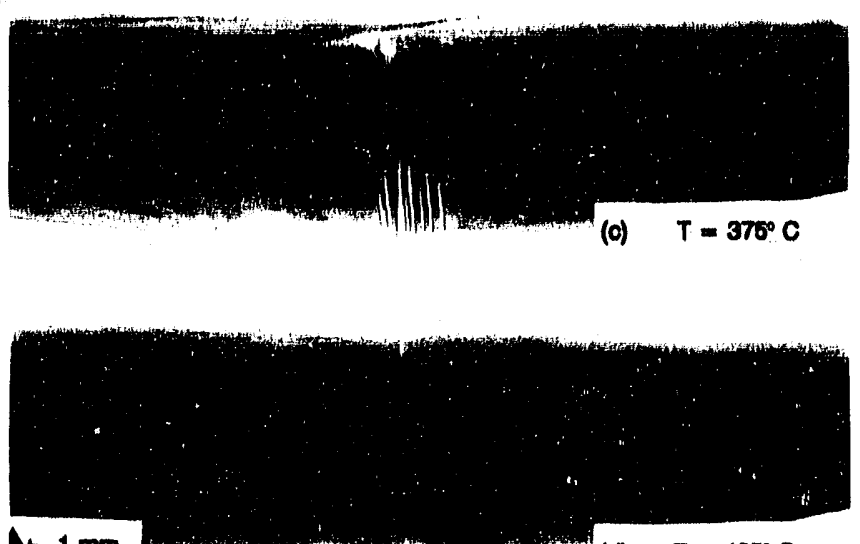

Ph $1 \mathrm{~mm}$ (d) $T=435^{\circ} \mathrm{C}$

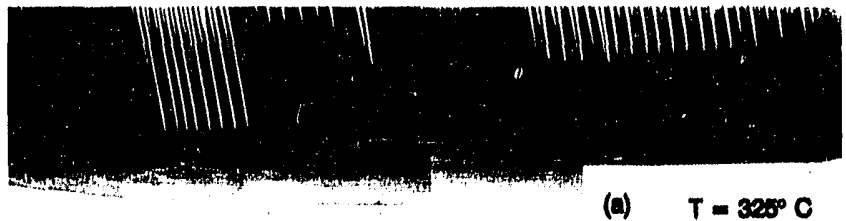

(a) $T=3000 \mathrm{C}$

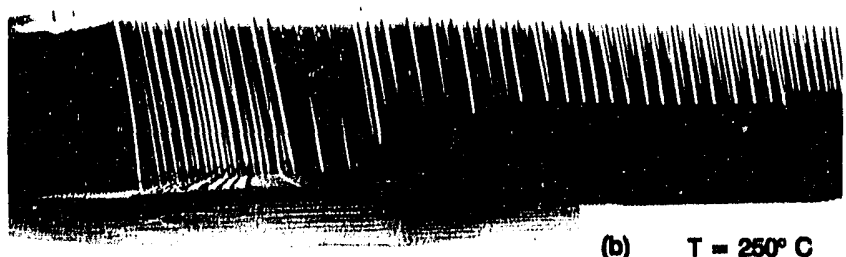

(b) $\mathrm{T}=260^{\circ} \mathrm{C}$
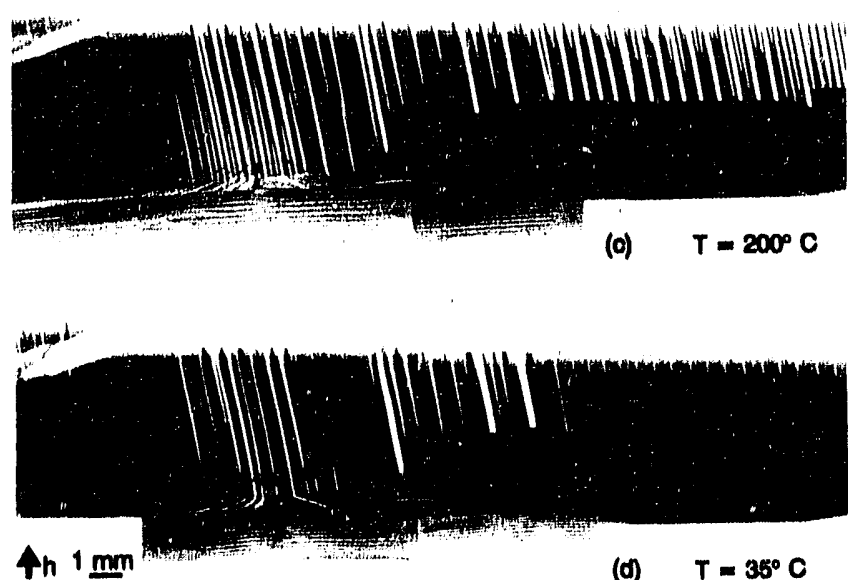

Figs. 45, 46 Synchrotron White Beam Reflection Topographs, $g=220, \lambda=0.98 \AA$, recorded from a LaAlO $\mathrm{L}_{3}$ single crystal as it undergoes a second order, rhombohedral-to-cubic phase transition at $435^{\circ} \mathrm{C}$. The temperature increases from $100^{\circ} \mathrm{C}$ to $435^{\circ} \mathrm{C}$ in Fig. 45 and decreases from $325^{\circ} \mathrm{C}$ to $35^{\circ} \mathrm{C}$ in Fig. 46. Note the (101) mirror twins in the room temperature rhombohedral phase (Fig. 45 (a)). These twin planes become mirror planes in the cubic phase so that the twins disappear as shown in Fig. 45 (d). As the temperature is subsequently lowered, these twins reappear as seen in Fig. 46 (a). Note that the twinning density is lower following a single cycle through the transition (Fig, 46 (d)). 

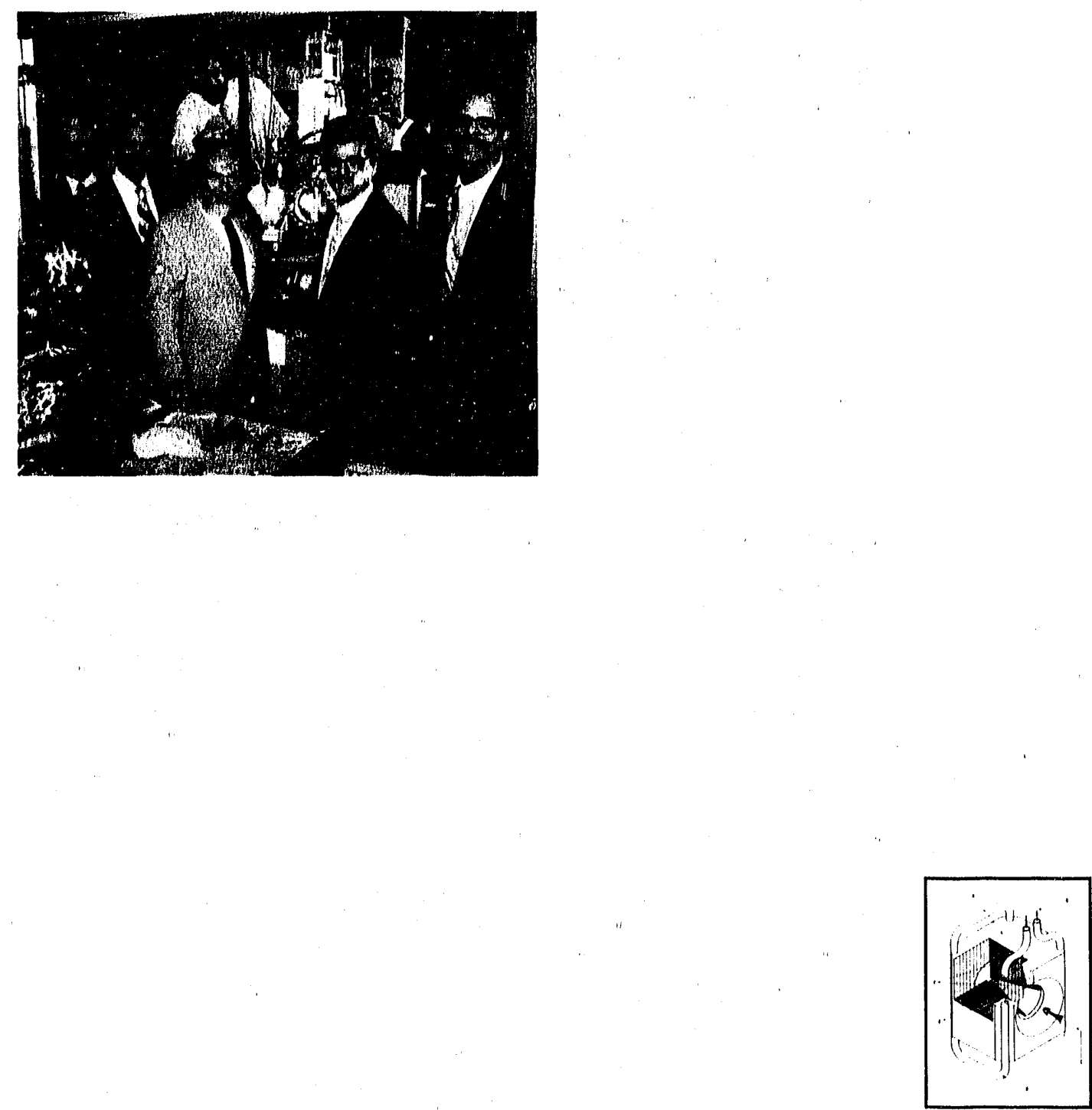

Section II Symposia, Workshops and Projects 
NSLS USERS'

MEETING

\section{Mark Rivers \\ U. of Chicago \\ UEC Chairman}

The twelfth NSLS Annual Users' Meeting was held on May 20-22, 1991. The first day was devoted to five workshops, which were attended by a total of 221 people. The workshop topics included:

Atomic and Molecular Science, chaired by Brant Johnson of Brookhaven National Laboratory. The workshop topics included photoionization studies of atomic ions and molecules, high resolution molecular core level photoelectron spectroscopy and studies of chemistry induced by core electron excitation.

Computational Tools in XAFS, chaired by Tim Morrison of the Illinois Institute of Technology. This workshup covered the use of $a b$ initio codes in modeling XANES and EXAFS data, standardization and criteria in EXAFS analysis, and ancillary tools in EXAFS analysis. The workshop concluded with a caucus on time-resolved EXAFS in the U.S.

Electronic and Chemical Phenomena at Surfaces, chaired by Kevin Smith of the University of Oregon and Neal Shinn of Sandia National Laboratory. This workshop highlighted recent results from the NSLS VUV Ring in the fields of two dimensional electronic struc- ture, adsorbate interactions and surface chemical reactions.

Imaging, chaired by Harald Ade and Shawn Williams, both of SUNY at Stony Brook. The topics covered in this workshop included microprobe instruments, such as scanning transmission and photoelectron microscopy, electron microprobes, and synchrotron $\mathrm{x}$-ray fluorescence microprobes. Also covered were projection imaging methods, such as angiography, microtomography and topography, as well as reciprocal space imaging techniques, such as x-ray holography and crystallography.

Surface Structure, chaired by Doon Gibbs of Brookhaven National Laboratory. This workshop focused on x-ray surface scattering studies. Talks included topics such as the phase behavior of $\mathrm{Au}$ and Pt surfaces, phase transitions of surface adlayers, and organic monolayers on water.

The main Users' Meeting began on Tuesday morning and was attended by 312 people. The invited keynote speaker, U.S. Congressman George Hochbrueckner, was unable to attend because of a Congressional session, but he sent a letter which was read by BNL Deputy Director Marty Blume. Hochbrueckner expressed his enthusiasm and support for such NSLS projects as $x$-ray lithography, coronary angiography, x-ray microprobe and microtomography and studies of catalysts using $\mathrm{x}$ ray scattering and photoemission spectroscopy.

Denis McWhan, the chairman of the NSLS, outlined the achieve- ments at the NSLS in the last year and plans for the future. A total of 1,950 users conducted experi. ments on the 29 beamlines on the VUV Ring and 56 beamlines on the X-ray Ring. The upgrades to the storage rings completed this year include the addition of a global feedback system to stabilize the vertical beam position in the X-ray Ring. Horizontal feedback will be accomplished in the coming year with the arrival of new pick-up electrode receivers. Upgrades in the accelerator control computer systems and the injection system are also planned or underway. McWhan outlined steps which have been taken to improve the working environment for NSLS users. These include improvements in electronic commun. ications and a decision to create a comfortable user lounge and library. He announced that a decision had been made to increase from one to three the number of Proposal Study Panels which review General User Proposals for experiments on the X-ray Ring. The new panels will cover the fields of Scattering/Crystallography, Spectroscopy and Imaging/ Other. McWhan also noted that the Insertion Device Beamlines are now reaching routine operations and are preparing to accept general user proposals. Users are encouraged to begin submitting proposals for experiments to be done on the U5 undl aator, the U13 wiggler, the $\mathrm{X} 1$ undulator, the $\mathrm{X} 13$ miniundulator, the $\mathrm{X} 17$ superconducting wiggler and the X25 hybrid wiggler. McWhan also discussed the issue of user fees which are being studied by the U.S. Department of Energy. These would require all users to 
pay for beam time at facilities such as the NSLS. McWhan stated that "If user fees are instituted it could kill DOE user facilities such as the Light Source. We are working with other facilities to make sure our arguments are heard." 'I'he topic of user fees was discussed further at the round table forum on Tuesday afternoon. Users were encouraged to make their feeling about this issue known in Washington. The NSLS Users' Executive Committee has written a letter to Admiral Watkins explaining their opposition to the proposed fees.

The scientific talks at the meeting included two talks on X-ray lithography. Jim Murphy discussed the status of the Phase I X-ray Lithography Source at the NSLS. This warm compact ring has met all of its design specifications with a maximum stored current of $1200 \mathrm{~mA}$ at $200 \mathrm{MeV}$. Currents as high as $250 \mathrm{~mA}$ have been achieved when injecting at the lower energy of $77 \mathrm{MeV}$. 'The superconducting magnets for the Phase II cold ring are now being fabricated. Rick Freeman of AT\&T Bell Labs reported on progress in $x$-ray projection lithography at the NSLS. Freeman considers projection lithography to be a promising technique for producing circuits with features around $100 \mathrm{~nm}$, which he believes will be required sometime after the year 2000 when chip densities reach 1 Gbit. Projection lithography uses $x$-ray optics to produce a demagnified image of the mask on the photoresist. Freeman stated that while the technique is very promising it will require an enormous investment to become com. mercially viable.

D. Peter Siddons discussed recent results of Mössbauer spectroscopy using synchrotron radiation. By using a very high resolution monochromator, his group has, for the first time, been able to do Mössbauer spectroscopy using synchrotron radiation on samples which were not perfect crystals. The advantages of synchrotron radiation for Mössbauer studies include its polarization and high intensity, which should permit the study of small samples. It also permits one to study nuclei which do not have a radioactive parent. Using existing synchrotron sources, however, it is only possible to study samples which have a very high concentration of the isotope of interest (such as isotopically enriched foils). The hope is that by using third generation synchrotron facilities it will be possible to do Mössbauer studies of nuclei at natural abundances.

Other talks covered soft x-ray spectroscopy of metal enzymes, coronary angiography, observa. tion of speckle with $\mathrm{x}$-rays, nuclear physics at the LEGS beamline, scattering from bulk liquids and thin wetting layers, photoelectron holography and time resolved studies of heterostructure interfaces.

Four new members were elected to the Users' Executive Committee. These are Paul Cowan (Argonne
National Laboratory), C.T. Chen (AT\&T Bell Labs), Gabrielle Long (NIST) and Larry Sorenson (Univ. of Washington). Neal Shinn was elected as Vice-Chairman, and will become Chairman at the Users' Meeting in 1992.

The meeting banquet was held on Tuesday night in Berkner Hall. Special awards were made this year in honor of two recent retirees from the Department of Energy. Chalmers Frazer was thanked for his years of service both at BNL where he served as a senior physicist and Deputy Chairman of the Physics Department, and in the Department of Energy where he served as Chief of the Solid State Physics and Materials Chemistry Branch. Don Stevens was honored for his tremendous efforts in the Depurtment of Energy which were critical in the development of both the current generation of synchrotron facilities, such as the NSLS, and also in starting the next generation, such as the Advanced Light Source at Berkeley and the Advance Photon Source at Argonne.

Other highlights of the meeting included a poster session, in which over 60 posters were presented, displaying research at the NSLS. A vendor display included $18 \mathrm{com}$ panies with products ranging from vacuum equipment to multilayer crystals to X-Windows terminals. 


\section{WORKSHOP ON SURFACE STRUCTURE}

\section{Doon Gibbs}

BNL Physics Department

$\mathrm{X}$-ray diffraction and reflectivity studies of solid and liquid surfaces and interfaces are a central component of the scientific program at the NSLS. The workshop on surface structure sampled the diversity of experiments now being performed on the floor of the X-ray Ring. Approximately seventy-five persons attended the one day meeting. The program follows:
I. Session Chair: Jacqueline Krim, Northeastern University

In Situ Surface X-ray Scattering of M. Toney, IBM Electrochemically Deposited Metal Monolayer

Structure of Electrified Interfaces $\quad$ B, Ocko, BNL

II. Session Chair: A.D. Navaco, Lafayette College

Phase Transitions of Surface $\quad$ K. Liang, Exxon Adlayers

High Temperature Studies of Metal E. Conrad, Univ. Surfaces $\quad$ of MO

Phase Behavior of $\mathrm{Au}$ and $\mathrm{Pt} \quad$ G. Grübel, BNL Surfaces

III. Session Chair: Lonny Berman, NSLS

Standing Wave Studies of X-ray P. Cowan, NIST

Semiconducting Surfaces and

Interfaces

2D Solid and Liquid $\mathrm{Pb}$ on $\mathrm{Si}(111) \quad$ E. Fontes, AT\&T

Coherence and Phase Separation of K. Blum, MIT

Steps on Si(111)

Steps and Step Bunching After Homoepitaxy on Si(001) Surfaces

P. Fuoss, AT\&T

IV. Session Chair: Sunil K. Sinha, Exxon

Organic Monolayers on Water

P. Dutta, Northwestern Univ.

Complete Wetting of a Rough I. Tidswell, Harvard Surface

2D Order Kinetics: Ising System in a Dilute Random Field
P. Eng, SUNY at Stony Brook 


\section{WORKSHOP ON ELECTRONIC AND CHEMICAL PHENOMENA}

\author{
Kevin E. Smith \\ Boston University \\ Chairman
}

Neal D. Shinn

Sandia National Lab

Co-Chair

A day-long workshop dealing with the area of electronic and chemical phenomena at surfaces was held in conjunction with the 1991 Annual Users Meeting. The goal of the workshop was to present, in a relatively informal manner, new results in this field obtained at the NSLS. The workshop consisted of a series of invited talks from members of the NSLS user community. The talks were targeted at a general surface physics and chemistry audience, and the schedule allowed plenty of time for questions and answers. This format worked very well. This meeting complemented a parallel session on surface atomic structure held at the same time.

The first technical presentation of the day was a lively talk given by
Peter Johnson (BNL - Physics, U5), who spoke on spin resolved photoemission studies of adsorption on ferromagnetic surfaces. With the audience now wide awake, the next talk was by $C$. T', Chen (AT\&T - Bell Labs, U4B) who presented a fascinating talk on the use of soft x-ray magnetic circular dichroism as a tool for studying magnetic phenomena at the surfaces of crystals and thin films. (This talk provoked a vig. orous question periodl). Moving further into the area of surface adsorbates, the third talk of the day was by Gwyn Williams (BNL NSLS, U4IR) who discussed the use of synchrotron generated infra. red radiation in the study of the coupling between adsorbate vibrational modes and the electronic structure of a metal si'rface. Fol. lowing a short coffee treak, Neal Shinn (Sandia National Lab, U3/ U16) brought the proceedings firmly into the area of surface chemistry with a discussion of the chemical properties of epitaxial metal overlayers. Jory Yarmoff (University of California at Riverside, U8) gave the final talk before lunch. Continuing the chemistry theme, his was a thought provoking presentation concerning the halogen etching of semiconductors.
Following lunch, the proceedings resumed with an almost philos. ophical discussion by Steve Kevan (University of Oregon, U4A) of time and energy scales at sur. faces. The difficult role of following Steve fell to Paul Bruhwiler (Un1versity of Pennsylvania, U12) who revisited the enduring topic of core level spectra from adsorbates on simple metal surfaces. Jack Rowe (AT\&T Bell Labs, U4A) continued the core level discussion by speaking next on the electronic structure of Si surfaces and interfaces. Following another break for caffeine, the penultimate talk was presented by Dave Heskett (University of Rhode Island, U12), who discussed the structure of $\mathrm{Bi}$ overlayers on III/V semiconductor surfaces. The technical section of the workshop ended with a talk by Eric Jensen (Brandeis University, U14/U7) on the ressults of the painfully low signal Auger-photoomission coincidence spectroscopy studies of solid surfaces. The workshop concluded with an open discussion on the future of the UV Ring, led by Neal Shinn, Gwyn Williams, and Kevin Smith. Overall, this workshop was very successful, was well attended, and was a measure of the vitality of the surface science community at the NSLS. 


\section{WORKSHOP ON IMAGING}

\section{Harald Ade \\ SUNY at Stony Brook \\ Chair}

\section{Shawn Williams}

SUNY at Stony Brook

\section{Co-Chair}

The increasing size, diversity and success of the X-ray imaging community at the NSLS revealed the necessity of bringing together experts from many fields with the common thread of imaging, to discuss the hardware and software techniques used to get the most information out of their samples. One measure of the success enjoyed by members of this com. munity might be represented by the two R\&D 100 awards won by imaging devices at the NSLS during the last few years. In addition to hardware and its applications, one of the workshop's goals was to help facilitate the transition towards more sophisticated and easier image analysis and presentation.

David Sayre opened the workshop, to provide a conceptually co" hesive framework, with a general talk delineating principles and concepts of imaging. Subsequent talks were arranged in groups according to the primary concept and conceptual complexity of the various techniques. Microprobe devices, such as scanning photoemission (H. Ade, SUNY at Stony Brook) and transmission microscopes (S. Williams, SUNY at Stony Brook), X-ray fluorescence microprobes (K. Jones, BNL), as well as electron beam microprobes (M. Foster, BNL) were presented in talks before the lunch break. While electron microprobes obvi. ously do not use the NSLS, its presentation was included in the workshop to provide insight into image processing and display techniques used by the electron microscopy community, with the intent to stimulate similar use of these techniques by the $x$-ray imaging community. This included, for example, a special way to color code concentration ratios of several components in a sample, which can then be presented in a single 2-D image.

During the lunch break, com. mercial vendors displayed hardcopy devices, and a small poster session enabled participants to display work not covered by talks. Projection imaging and related techniques, such as angiography (D. Chapman, NSLS), topography (M. Dudley, SUNY at Stony Brook) and microtomography (J. Dunsmuir, EXXON) were presented in the first afternoon ses-

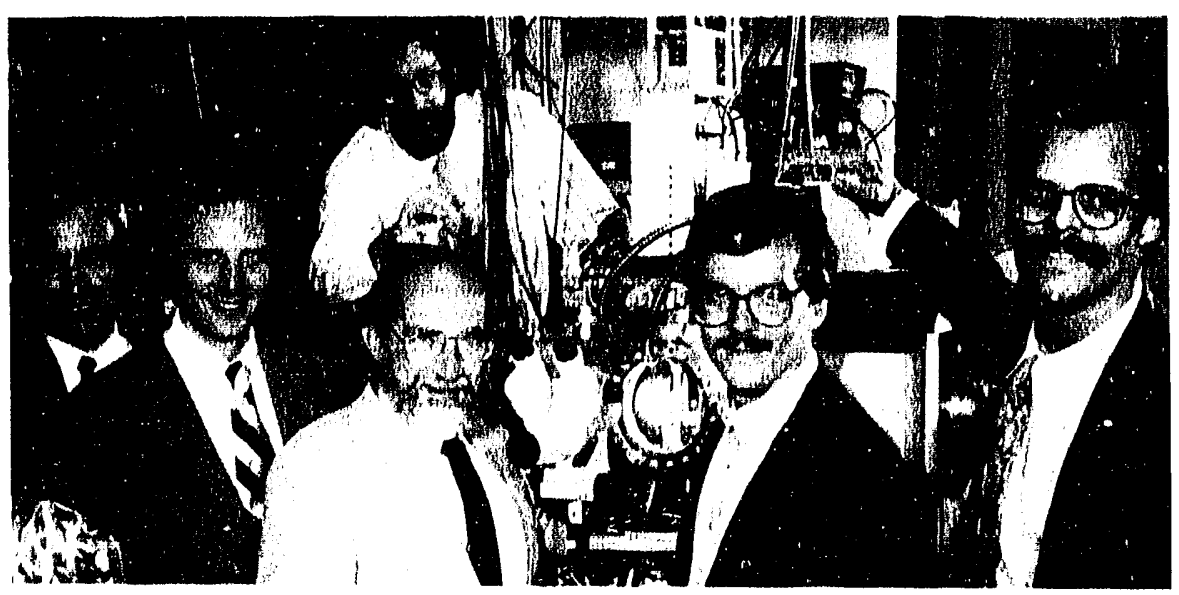

A 1991 R\&D 100 Award was given for the Scanning Photoemission Microscope (SPEM) developed by researchers from SUNY at Stony Brook and BNL. The collaborators were: Wrik Johnson, NSLS (rear); Dleter Kern, IBM (left); Erik Anderson, IBL; J anos Kirz, SUNY at Stony Brook; Steven Hulbert, NSLS; and Harald Ade, SUNY at Stony Brook. 


\section{UV FEL MACHINE REVIEWS}

\section{Ilan Ben-Zyi \\ NSLS}

Sam Krinsky
NSLS
Deputy Chairman

The National Synchrotron Light Source is preparing a proposal for the construction of a linac based Free-Electron Laser (FEL) (see "Proposed UV-FEL User Facility at the NSLS" in Section IV). An advisory panel has been asked to review the FEL design and guide the NSLS team in the associated scientific and technological issues. The panel members are:
J. J. Bisognano - CEBAF
J. S. Goldstein - LANL
C. W. Leemann - CEBAF
J. M. J. Madey - Duke
R. Miller - SLAC
E. T. Scharlemann - LLL
H. A. Schwettman - Stanford
A. M. Sessler - LBL (Chair)
R. Sheffield - LANL
J. Wurtele - MIT

The advisory panel held two meetings in 1991. The first meeting took place on April 11-12. Presentations by the BNL staff outlined the design of a UV FEL to provide a high peak power, tunable, coherent source from the visible down to $100 \mathrm{~nm}$, with the possibility for operations at $50 \mathrm{~nm}$ and below, with further development. "In order to provide multiple radiation sources, switching of the electron beam to different wigglers at kilohertz rates is pro- posed based upon transverse separation due to energy modulation and dispersion. Moreover, the output of a single FEL is divided between several users using a rotating mirror."

The presentations included welcome and charge: D. McWhan and S. Krinsky, system overview: I. Ben-Zvi, FEL design: L. H. Yu, RF gun: K. Batchelor, seed laser: L. DiMauro, transport and recirculation: S. Kramer and G. Ingold, linac and cryogenics: $H$. Halama and M. Iarocci, wiggler: L. Solomon and G. Ingold.

The panel examined the key design choices of the machine and has confirmed the NSLS team's choices. In their report they said, "The choice of a superconducting linac as the driver of the source offers unique opportunities in terms of electron beam quality, and flexibility of the pulse time structure and repetition rate... We strongly support the choice of a superconducting RF linac as the driver for a user facility UV FEL source." The panel ulso supported the choice of operating temperature and frequency of the linac. Other issues that were examined were the choice of an FEL amplifier over FEL oscillator and the proposed use of frequency multiplication in the FEL.

In general, the panel felt that "...the design has innovative features that should be pursued to clarify their full potential." The advisory panel made a list of recommendations covering issues of the superconducting linac, beam optics, emittance preservation, FEL physics, wiggler design and more. It was stated that "...there are many items which require further work prior to a Proposal. The Panel is interested in following the development of these ideas and has agreed to meet again at the NSLS on October 21-22, 199 .."

In the October meeting the program started again with presentations by the NSLS staff: welcome and charge: S. Krinsky, project overview: I. Ben-Zvi, FEL design: L. H. Yu, Scientific case: E. Johnson, electron gun: K. Batchelor, electron transport: S. Kramer and $\mathrm{X}$. Zhang, linac: H. Halama, lasers: A. Fisher, diagnostics: J. Rogers, wiggler: G. Ingold, wiggler errors: A. Friedman. The reaction of the panel was again positive: "The panel was very favorably impressed with the progress that has been made since our meeting in April. In particular we are pleased to note that significant progress has been made on the scientific case for the proposed facility."

The panel made a series of comments on the draft CDR, recommending a cost estimate to be developed, further design of the recirculating arcs, reduction of the sensitivity of the FEL to parameters, increased injection energy, capability of higher average linac beam current, sizing magnets for higher gradients in the linac, magnetic beam bunching study of beam optics of the superconducting carities, wake field policy, study of the beam matching to both undulators, treatment facility for cavities, beam stability, beam diagnostics, control systems and a commissioning plan. 
The panel focused its deliberations upon pre-construction R\&D. After much deliberation the panel was of the opinion that there are approximately eight items that should be addressed before embarking on the construction project. Most of these items are being addressed in the R\&D program of the Accelerator Test Facility (ATF) (see "Accelerator Test Fa- cility" in Section IV). The precontruction R\&D items are:

1. High duty factor RF gun.

2. Matching of gun into ATF linac and subsequent acceleration.

3. Superconducting cavities control.
4. Visible FEL experiment on the ATF.

5. Harmonic generation experiment on the ATF.

6. Construction and measurement of prototype undulator and diagnostics.

7. Seed laser demonstration.

8. Photocathode gun laser demonstration. 
DOE

HIGH SCHOOL

HONORS

RESEARCH

PROGRAM

William Lynch

$B N L$ Office of Educational

Programs

The Department of Energy High School Honors Research Program, now in its fifth year at Brookhaven National Laboratory, was host to 60 high school students this year. The two week program, held between July 24 and August 6 , was designed to provide a top high school student from each state access to several of the premier research machines at the Laboratory, as well as to expose them to the day-to-day "business" of scientific research. In addition to the 50 states, a student from the District of Columbia, Puerto Rico, the Department of Defense Dependent Schools, and seven foreign countries (Canada, England, France, Italy, Japan, Mexico, and Germany) attended this year's program.

The first two full days of the program were devoted to familiarizing students with the NSLS and the BNL environment. They heard lectures on safety ( $T$. Dickinson), properties of synchrotron radiation (S. Hulbert), applications of synchrotron radiation (R. Klaffky), and relativity (J. Rogers). Sessions were also held to discuss physics in general and the specific experiments the students would be doing at the
NSLS and the Department of Applied Science.

Sessions were also held to meet and work with their team members, as well as to apply their new knowledge. The students worked in ten groups of six students each, to calculate the theoretical photon flux output of the NSLS at two different photon energies. In addition, the students toured both the NSLS and the Laboratory site during the initial two days. After a day's visit to New York City, the next three days were devoted to running their experiments. Thanks to the combined efforts of the scientific staff and the Office of Educational Programs, there was a total of 6 experiments available for the students to do. A total of 26 three-hour shifts at the NSLS, and 4 threehour shifts at the Department of Applied Science enabled each of the 10 teams of students to perform 3 different experiments. The six 1991 experiments are de. scribed below.

Experiment XXX: Optical Diffraction (D. Chapman/N. Lazarz). This experiment used a $\mathrm{He}-\mathrm{Ne}$ laser and simple objects to introduce principles of diffraction from periodic structures and computer controlled data acquisition. The students measured the wavelength of the $\mathrm{He}-\mathrm{Ne}$ laser light with a machinist's ruler and using this data, determined the spacing of a Ronchi ruling.

Experiment U4-IR:Fourier Transform Infrared Spectroscopy (G. Williams). The students studied the operation of the FTIR on U4IR and obtained spectra of glass, black polyethylene film, potas- sium bromide, potassium iodide and barium fluoride. The students then determined the "spring constant" for the ionic compounds and observed how well the measured data fit a simplified theory of atomic vibration.

Experiment U-14: Monochromator Efficiency and the Photoelectric Effect (C.-C. Kao). The students determined the monochromator transmission efficiency between 100 and $300 \mathrm{eV}$. This was done by measuring the photoelectric current generated by illuminating a metal with a known photoelectron yield with light from the NSLS. The calculated flux from this measurement (monochromator output) was compared to the theoretic photon flux (monochromator input).

Experiment X-26: Trace Element Analysis by X-Ray Fluorescence (K. Jones). Students bombarded a known sample with "white" $x$. rays and observed the fluorescence peaks corresponding to the different trace elements in the sample. They then applied their new skills by trying to identify the "perpetrator" at a "crime scene" by performing a trace element analysis on a hair sample from the "crime scene." They compared this data to the elemental analysis of each team member's hair to identify the unknown "perpetrator."

Experiment X-19: Oxygen Absorption in Hemoglobin (L. Furenlid). Students determined the change in the iron $\mathrm{K}$-electron binding energy as a function of oxygen loading and used this relationship to predict the $\mathrm{K}$-shift for partial oxygen loadings. 
FP-ST: Shock Tube Studies of Reaction Kinetics (B. Klemm/J. Sutherland). Students used the Flash Photolysis - Shock Tube apparatus in the Department of Applied Science to determine the rate constant for the reaction between 1-butene and atomic oxygen at a pressure of about 600 torr and a temperature of approximately $1200 \mathrm{~K}$. The operation and capabilities of the apparatus were also thoroughly investigated.

During the three days that the experiments were being done, the students who were not at the NSLS or the Department of Applied Science taking data, wrote the Experiment Reports for the completed experiments. Ten members of the scientific staff (mentors), then reviewed these first dratts, which were returned to the teams with comments. The mentors were: J. Smalley, DAS; B. Johnson, DAS; M. Bhat, DNE; A. Davenport, DAS; A. Sedlacek,
Chemistry; M. Rivers, DAS; S. Feldberg, DAS; R. Beuhler, Chemistry; C. Thorn, Physics; R. Thomas, OEP. After their first drafts were completed, the students toured five of the BNL departments (Chemistry, Applied Science, Medical Physics, and the HFBR), to expose them to the comprehensive research programs undertaken at the laboratory. In addition, during the week, the students heard two special lectures (DNA Damage and Repair Mechanisms - R. Setlow and Global Climate Change - B. Manowitz).

During the entire time that the students were at BNL, there were six program advisors available 24 hours a day to assist the students in all aspects of the scientific program as well as to provide supervision. The six program advisors were local high school teachers who lived with the students on the laboratory grounds for their two weeks stay. The advisors were: J. Callaway, E. Islip H. S.; L. Celenza, Central Islip H. S.; L. Haman, Dayton Avenue School C. Hudson, Central Islip H. S.; N. Coggins Lynch, E. Islip H. S.; and W. Lynch, E. Islip H. S.)

After a weekend spent visiting Port Jefferson or a water amusement park and then the beach at Watch Hill, the students formally presented their papers to their peers, their mentors, the program advisors and the scientific staff. On their last evening at BNL, a formal awards ceremony was held in Berkner Hall, with each student receiving a laser-engraved plaque commemorating their participation in the program. Information regarding the program and copies of the student reports or a videotape of their formal presentations can be obtained by contacting Karl Swyler or Don Metz in the Office of Educational Programs (516) 282-7171.

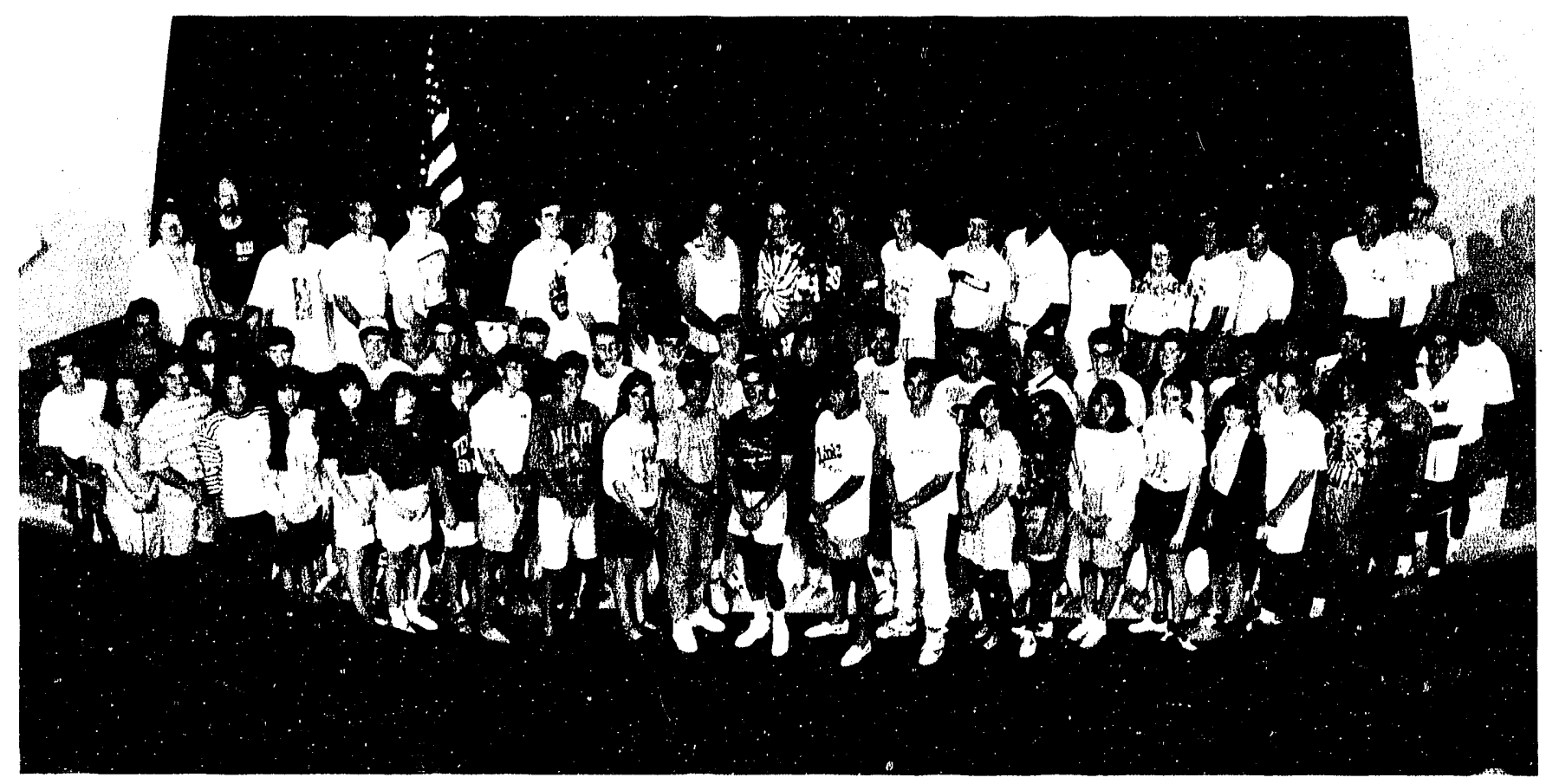




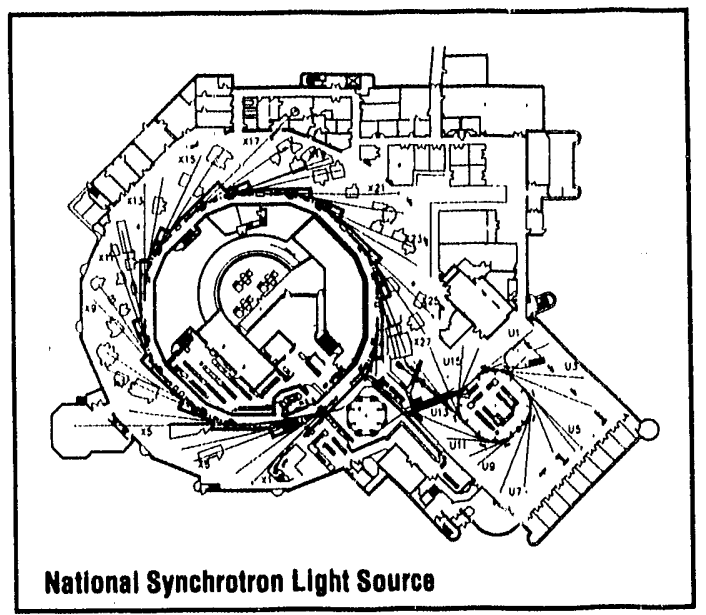




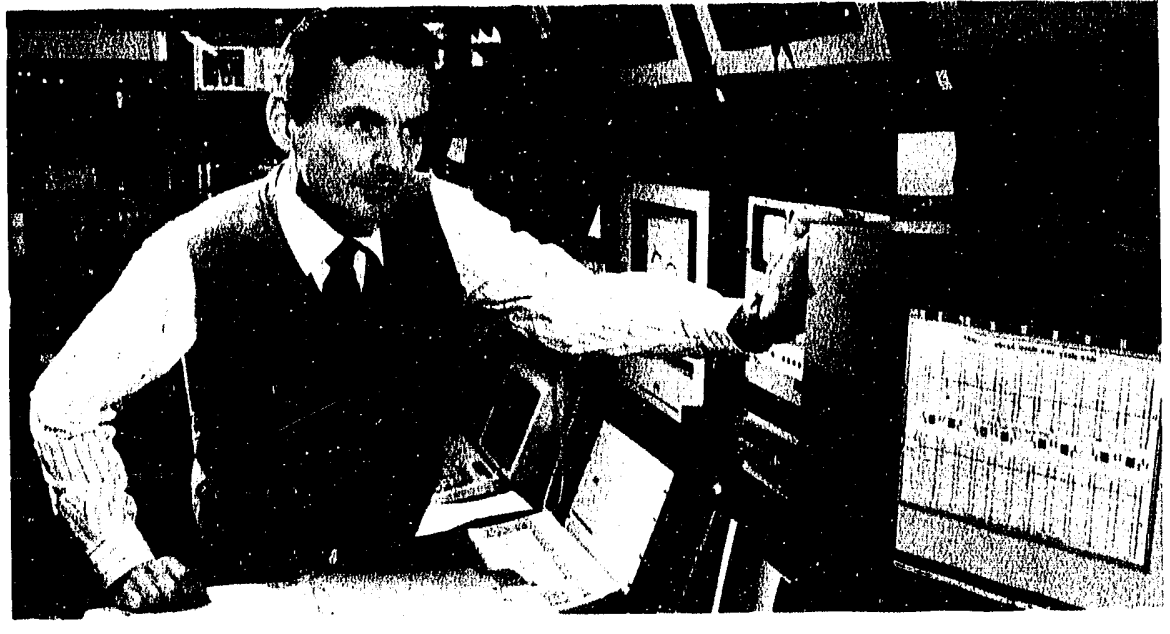

VUV MACHINE OPERATIONS

\section{Stephen Kramer NSLS \\ VUV Ring Manager}

The average VUV ring perform. ance statistics for FY 199l are given below:

Average fill current

- $837 \mathrm{~mA}$.

Average charge rate

- $38 \mathrm{~mA} / \mathrm{min}$.

Average lifetime at $500 \mathrm{~mA}$ -200 min.

Total user integrated current - 2480 A-hours (103.3 A-days)

Although the charge rate and fill current are down from last year's, the total integrated current delivered to the users in. creased slightly. With the major effort being expended on improving the injection system, the charge rate increased to $70 \mathrm{~mA} /$ min. at year's end with a slope that should exceed $90 \mathrm{~mA} / \mathrm{min}$. in the future.
Despite these problems, the integrated current exceeded last year's record value. This was accomplished with a $10 \%$ reduction in beam time available to the us. ers. A major reason for this drop in beam time was the unfortunate vacuum accident which vented the ring to 6 torr in October 1990. The subsequent repair, conditioning, and studies required to return the ring to normal operating conditions took away from the available beam time. The vacuum history showed recovery continuing through April 1991. The increased demand from the users for special low current and bunch number operation also contributed to the reduced integrated current. This makes the integrated current, as a figure of merit, not completely representative of the productivity for an active research machine.

The major improvement on the machine has been the commissioning of the 4th harmonic rf cavity. Although this cavity is presently operated without a power source, the bunch lengthening achieved with this cavity has resulted in a factor of two increases in beam lifetime at the higher currents.
Since the bunch lengthening is current dependent, the effect on the integrated current over a fill period is about $30 \%$. Powering this cavity to achieve optimum lengthening at all currents will be pursued in the future, when the resources are available to handle the phase locking of this system to the beam signal.

During this next fiscal year the major improvement will be the commissioning of new global feedback systems that will provide stabilization of the beam centroid in both the horizontal and vertical planes. Like the existing vertical global feedback system, the new system will damp beam oscillations in both vertical and horizon. tal planes, up to a frequency of 60 $\mathrm{Hz}$, by factors of two to five. The new system will also provide higher dynamic range from the new $r f$ receivers which will also be installed. In addition, with the doubling of the number of beam input channels, the new system will also damp beam oscillations that have correlations of their spatial sources that are different than the betatron tune number.

The global feedback systems will be able to handle the dynamic changes in the closed orbit. However, it doesn't address the reproducibility of the orbit from cold (zero current) fills or after shutdowns. Considerable effort will be made to track and eliminate the sources of these errors. The improved modeling of the ring which will result from these studies will also make it possible to consider user access to tuning the undulator gaps or photon energy. The new control system will provide 
the ability for user accessible macros that will not only vary the gap but also provide the steering and focusing adjustments of the electron beam that will make these changes transparent to the other users.

Studies are also planned on better understanding and improvement of the injection process. If significant improvement can be made on injection efficiency and beam sta. bility during the injection, then it may be possible to start to consider a possible top-off mode of injection. In addition to the obvious gain in integrated current, the topoff mode could stabilize the power level and density on mirrors and optical elements, in theory contributing to improved performance.

Once these efforts of improved performance of orbit stability, orbit reproducibility, and injection are achieved, there will be a need for user input to set priorities for future upgrades. Some of the potential improvements are:

1) - higher energy operation up to $800 \mathrm{MeV}$

2) - higher brightness beams

3) - greater flexibility in undulator tuning

However, the greatest potential may rest with the users, in proposing innovative photon beams and beamlines which will continue to keep the VUV Ring doing leading edge research well into the next century.
FY91 NSLS VUV RING

INJECTION/UNSCHEDULED DOWNTIME $(9.1 \%)$

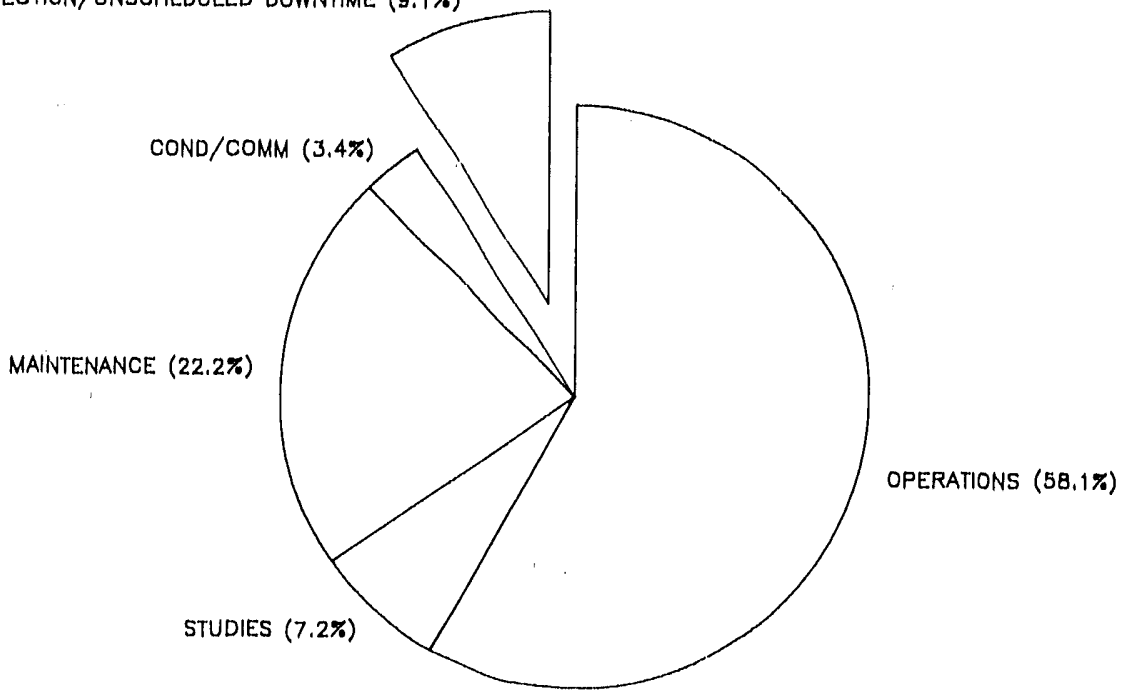

Fig. 1 VUV Ring time usage based on total time (not scheduled time) for fiscal year 1991.

$\frac{n}{0}$
$\frac{0}{5}$
0
1
0
$\frac{a}{4}$

Fig. 2 The VUV Ring integrated beam current available to users per month for fiscal year 1991. 
Fig. 3 The injection current averaged over all fills of the VUV Ring during each month of 1991 .

Fig. 4 The VUV Ring injection charge rate averaged over all fills during each month of FY 1991

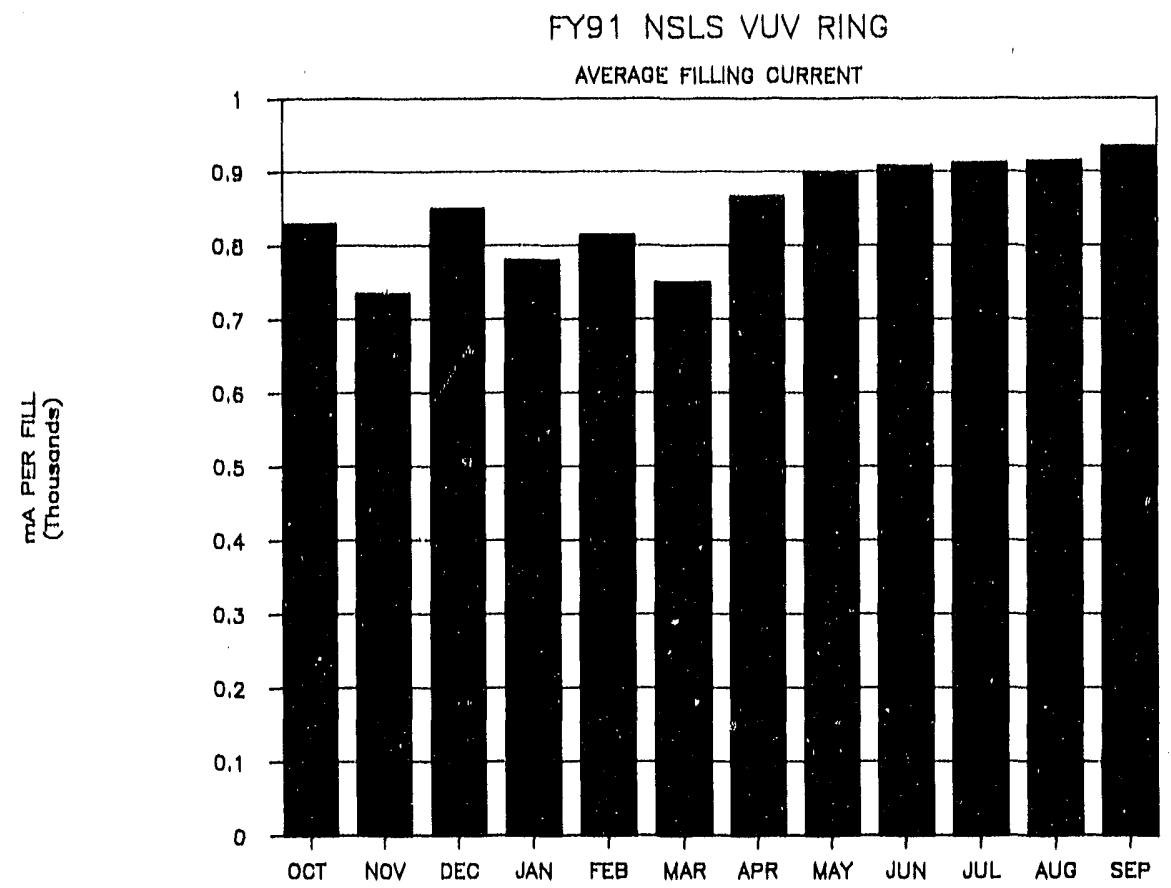

FY91 NSLS VUV RING

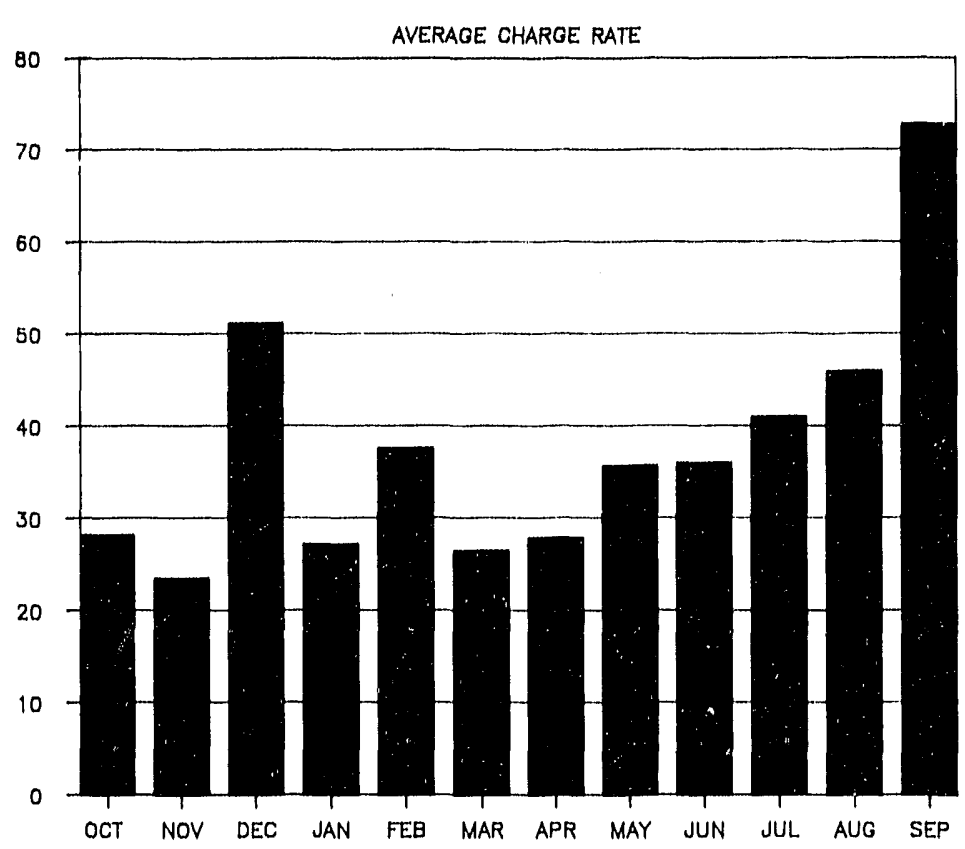


Fig, 5 The VUV Ring vacuum pressure at $500 \mathrm{~mA}$ beam current averaged over each month during FY 1991.

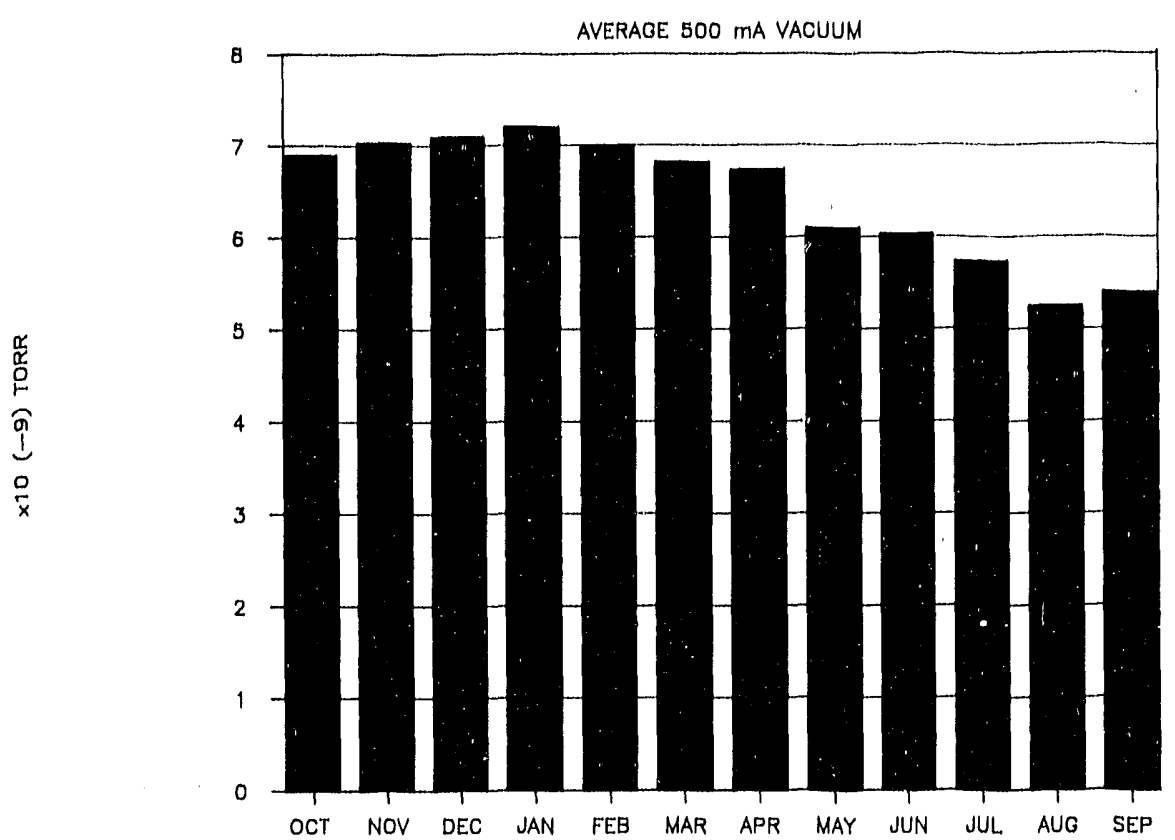

Fig. 6 The VUV Ring exponential beam lifetime at $500 \mathrm{~mA}$ beam current averaged over each month during FY 1991. The increased lifetime due to the harmonic cavity is evident beginning in December 1990.

FY91 NSLS VUV RING

AVERAGE $500 \mathrm{~mA}$ LIFETIME

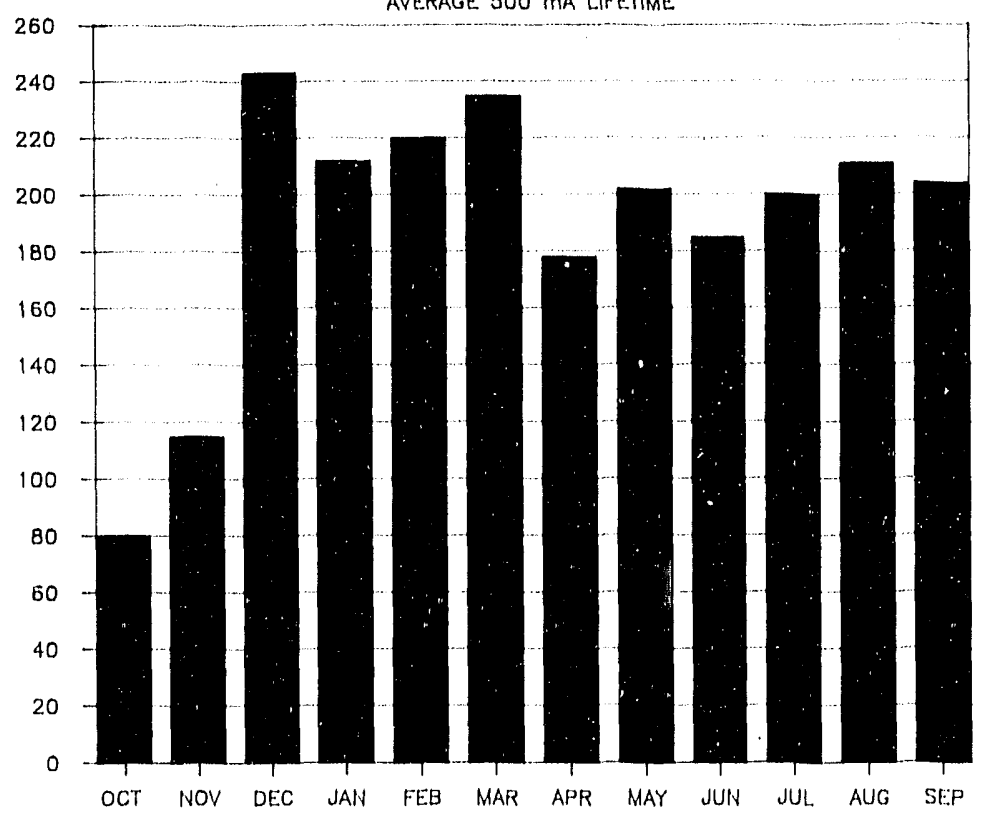




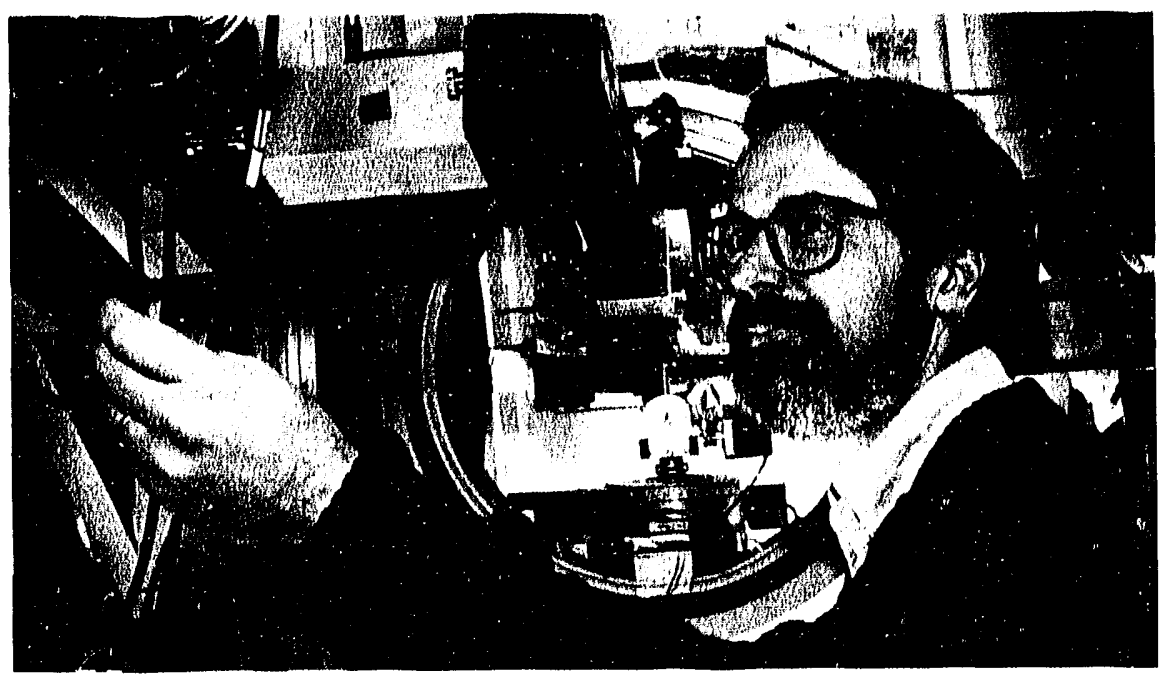

\section{VUV BEAMLINE OPERATIONS}

\section{Roger Klaffky \\ NSLS \\ VUV Operations Manager}

Over the course of FY 1991 there were 247 different experiments performed, representing a $27 \%$ increase over FY 1990. The institutional breakdown of scientists participating on these: experiments was $42 \%$ from universities, $21 \%$ from industry, $23 \%$ from BNL, $6 \%$ from other DOE labs, $4 \%$ from government labs, and $4 \%$ from abroad.

Major progress occurred in the U13UA high resolution/high flux soft $x$-ray spectroscopy branch line with the performance of the first gas phase experiments. On the U5U spin-resolved photoemis. sion beamline a new TGM/SGM monochromator was aligned and an MBE facility was connected to the experimental chamber. In addition, there were numerous beamline upgrades, as discussed below.
On the U2 lithography beamline, beam uniformity was improved by replacing a collimating mirror with a flat mirror and by installing a more uniform beryllium window. A new $\mathrm{M}_{0}$ mirror with improved figure was installed on the U3A ERG beamline to achieve a better focus, and a more accurately calibrated grating was installed. A preparation chamber was installed on top of the U4A experimental chamber to remove the need for a sample transfer mechanism. Also, a program was undertaken to improve the analyzer resolution by upgrading the detecmicroscope were commissioned; pump-probe experimental capability was added using Nd.YAG light transported from U10 through a fiber optical cable. An additional beryllium and polyimide window were added to U6 to allow lithographic exposures at atmospheric pressure. A variable line-spaced high flux grating monochrom. ator operating from $50-220 \mathrm{eV}$ with a resolving power of 500 was installed on U10A. The toroidal focussing and scanning mirror was repolished and recoated with nickel. On U10B a rotatable extor. On the U4IR line a midrange IR $(0.5-8 \mu \mathrm{m})$ interferometer and perimental chamber was installed for pilotodesorption studies at varying incident angle. On U11 design work for a second order sort. ing and beam steering chamber was completed and a new mono. chromator controller installed. A time-of-flight gas phase cluster experimental chamber was put into operation on $\mathrm{U} 12 \mathrm{~B}$ and alignment of U12A continued. On U14B simultaneous logging of vertical beam position, temperature of alu. minum water system (which cools the storage ring chamber), and $X$ and $Y$ displacements of PUE 20 was implemented. The U15 beam. line added the capability of detecting near IR to near UV luminescence from a large solid angle surrounding the emission region of decay products from core ex. cited free molecules. A sample cry. ostat for fluorescence EXAFS between room temperature and $40 \mathrm{~K}$ was also put into operation. The optical components on U16B were plasma cleaned using water vapor.

There were a number of changes on the UV floor. A new 40 ton air conditioning unit was installed on the roof, a stainless steel toxic gas exhaust system was installed for $\mathrm{U} 11, \mathrm{U} 14$, and $\mathrm{U} 15$, and the pump exhaust system was rerouted to remove it from beamline cable trays. A platform was built on the crane support beam to provide access for repairing components near the roof. An upgrade of beam. line electrical distribution sys" tems, an NSLS funded project, began. A new emergency generator was installed and wired into electrical distribution panels. Another major project was the installation of a new VUV roof. 


\begin{tabular}{|c|c|}
\hline \multicolumn{2}{|c|}{ VUV Storage Ring Parameters as of January 1992} \\
\hline Parameters & VUV Storage Ring \\
\hline Normal Operating Energy & $0.745 \mathrm{GeV}$ \\
\hline Maximum Operating Current (multibunch operation) & $1.0 \mathrm{amp}\left(1.1 \times 10^{12} \mathrm{e}^{-}\right)$ \\
\hline Circumference & 51.0 moters \\
\hline Number of Beam Ports on Dipoles & 17 \\
\hline Number of Insertion Devices & 2 \\
\hline Maximum Length of Insertion Devices & $\sim 2.5$ meters \\
\hline$\lambda_{c}\left(E_{c}\right)$ & $25.3 \mathrm{~A}(486 \mathrm{cV})$ \\
\hline$B(\rho)$ & 1.28 Tesla (1.91 meters) \\
\hline Electron Orbital Period & 170.2 nanoseconds \\
\hline Damping Times & $\tau_{y} \cong \tau_{y} \cong 17 \mathrm{msec} ; \tau_{g} \cong 9 \mathrm{msec}$ \\
\hline $\begin{array}{l}\text { Touschek lifetime dependent on current per } \\
\text { bunch and vertical emittance }\end{array}$ & $200 \mathrm{~min} @ 200 \mathrm{~mA}$ \\
\hline Lattice Structure (Chasman-Green) & Separated Function, Quad, Doublets \\
\hline Number of Superperiods & (1) \\
\hline Magnet Complement & $\begin{array}{l}8 \text { Bending ( } 1.5 \text { meters each) } \\
24 \text { Quadrupole ( } 0.3 \text { meters each) } \\
12 \text { Sexlupole ( } 0.2 \text { meters cach })\end{array}$ \\
\hline Nominal Tunes $v_{x}, v_{y}$ & $3.14,1.20$ \\
\hline Momentum Compaction & 0.023 \\
\hline R.F. Frequency & $52.887 \mathrm{MHz}$ \\
\hline Radiated Power & $14.7 \mathrm{~kW} / \mathrm{amp}$ of Beam \\
\hline R.F. Peak Voltage (typical) & $100 \mathrm{kV}$ \\
\hline Design R.F. Power & $50 \mathrm{~kW}$ \\
\hline$v_{s}$ (Synchrotron Tune) & 0.002 \\
\hline Natural Energy Spread $\left(\sigma_{\varepsilon} / \mathrm{E}_{*}\right)^{* *}$ & $4.5 \times 10^{-4}(\mathrm{I}<20 \mathrm{~mA})$ \\
\hline Natural Bunch Length $(2 \sigma)$ & $7.6 \mathrm{~cm}(\mathrm{I}<20 \mathrm{~mA})$ \\
\hline Number of RF Buckets & 9 \\
\hline Typical Bunch Mode & 7 \\
\hline Horizontal Damped Ernittance $\left(\varepsilon_{\mathrm{x}}\right)$ & $1.5 \times 10^{-7}$ meter-radian \\
\hline Vertical Damped Emittance $\left(\varepsilon_{\mathrm{y}}\right)^{x}$ & $\geq 2.8 \times 10^{-10}$ meter-radian (adjust.) \\
\hline Power per Horizontal milliradian, 1A & 2.3 Watts \\
\hline Source Size: $\sigma_{h}, \sigma_{v}$ & $0.5 \mathrm{~mm},>0.06 \mathrm{~mm}$ \\
\hline $\begin{array}{l}\text { Lifetime variable up to }-350 \text { minutes depending on } \\
\text { lengthening } 211 \mathrm{MHz} \text { RF cavity. }\end{array}$ & \\
\hline ** Current and RF voltage dependent. & \\
\hline
\end{tabular}




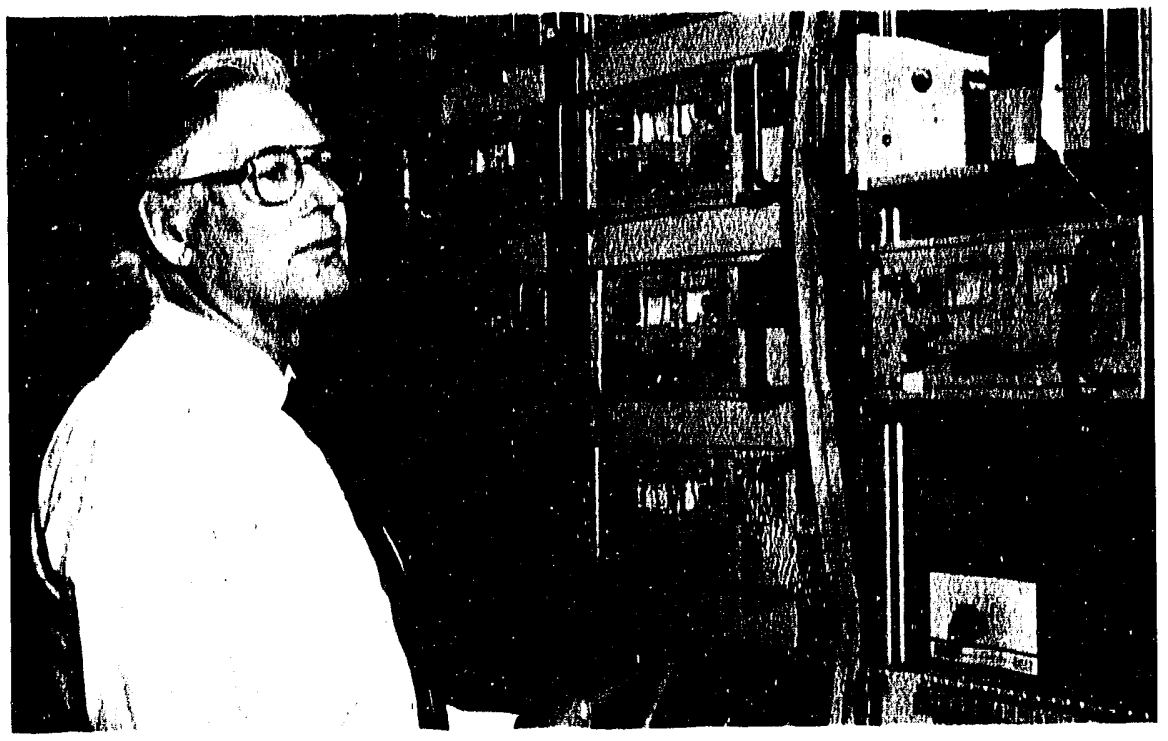

\section{X-RAY MACHINE OPERATIONS}

\section{Norman Fewell \\ NSLS \\ $X$-ray Ring Manager}

Commissioning of the high power insertion devices has continued during this period. Full power operation of X17 and X25 is now routine. The active interlock system for X21 is now complete, and this beamline is expected to come on line shortly. Local feedback systems for X1，X5, X17, and X25 have been developed and are fully operational.

Beam stability has been greatly improved by the installation of a real time harmonic global feedback system. 'This system, which operates in both planes, is similar to that developed for the VUV Ring, for which the designers won a R\&D 100 award. The installation of this feedback system, which has to function without ad- nant frequency of the quadrupole/ support girder combinations. Some magnet movement was reduced by tightening support bolts and regrouting some floor attachments. Further reduction was done by moving the resonant frequency of the quad/girder combinations. This was achieved by supporting the large amount of lead shielding on newly con. structed supports, instead of on the quadrupole support girders. These changes reduced the beam movement sufficiently, such that the compressor could run without noticeable movement by the X-ray users, and since the vibration frequency lies within the range of the newly installed global feedback system, the future operation of the HFBR compressor should not be a problem.

Installation of the $\mathrm{RF}$ receivers used by the feedback systems continues. At the present time, 26 units out of a full complement of 48 have been installed and are operational. When completed this system will allow real time orbit measurements. The Computer Group has developed a system that displays the X-ray orbit, using the present complement of receivers, at a display rate of ap. proximately 5 orbits/sec.

An investigation into methods to reduce beam motion caused by operation of the HFBR helium compressor was carried out by a private consulting company and BNL staff. It was found that the magnitude of the motion was dependent on the water saturation levels of the ground between the HFBR and the NSLS. It was also found that the compressor frequency (approximately $12 \mathrm{~Hz}$ ) $\mathrm{c}^{\circ}$ incided with the vibrational reso-
Some of the problems encountered during the year include the vacuum failure of a RF cavity power coupling window, and the failure of a high voltage transformer. This transformer weighs approximately $2,000 \mathrm{lbs}$. and is located inside the RF penthouse on top of the roof, which posed quite a logistics problem. In addition to down. time due to normal equipment failures, this year we were plagued by a series of computer prohlems that led to approximately $20 \%$ of our unscheduled downtime. A concerted effort by the Computer Group finally alleviated the problem and the computer system is now working adequately and work on upgrading the system is progressing. 'The installation of a large air conditioning unit and ductwork in the power supply area has made a marked improvement in the temperature of that area. 
Previously, inadequate cooling had led to extremely high ambi nnt and equipment operating temper" atures which could lead to equip. ment malfunction or failure.

On the whole this was a good run. ning year for the X-ray Ring, and with most of our commissioning bugs behind us, we look forward to improved performance in the coming year.
FY91 NSLS XRAY RING RINO TIMEE USAOE

INJEOTION/UNSCHEDULED DOWNTIME (9.8\%)

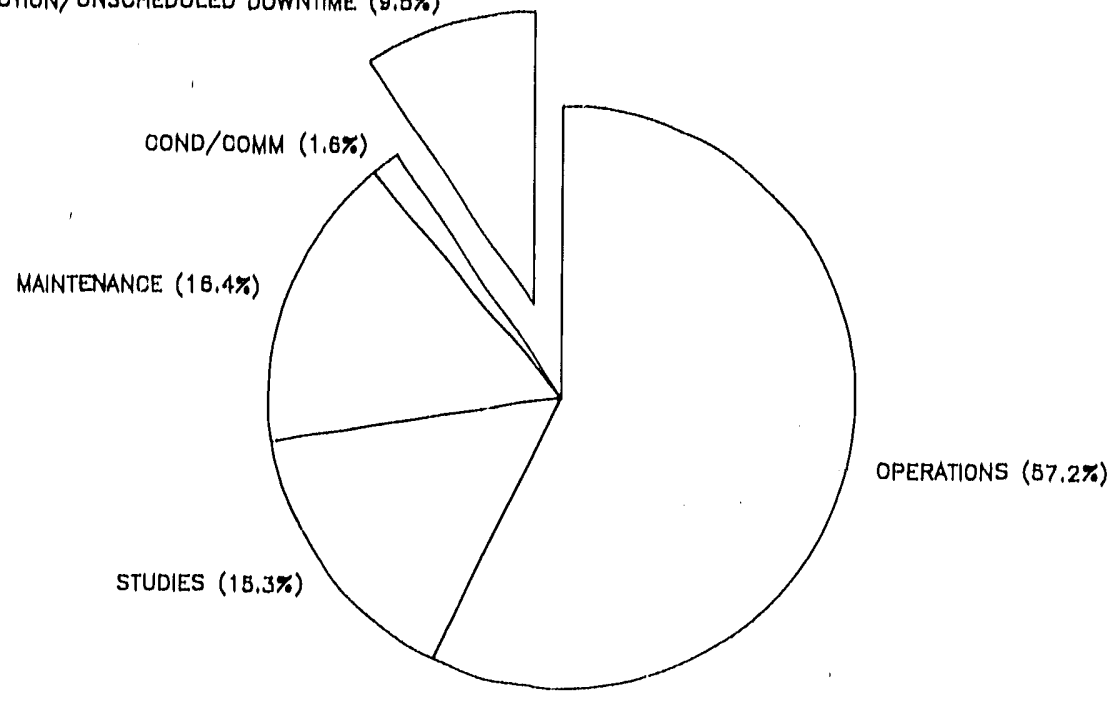

Fig. 1 X.ray Ring time usage based on total time (not scheduled time) for fiscal year 1991.

FY91 NSLS XRAY RING

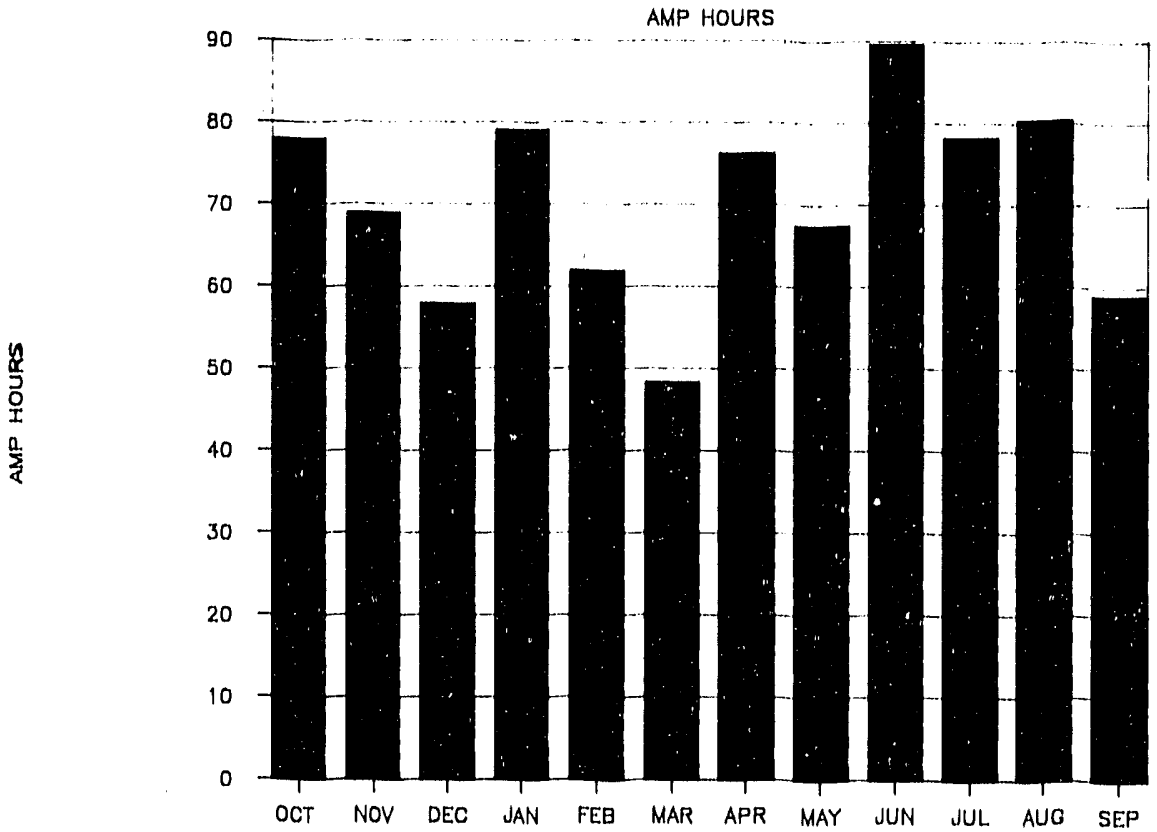

Fig. 2 The X-ray Ring integrated beam current avallable to users per month for fiscal year 1991. 
Fig, 3 The infection current averaged over all fills of the X-ray Ring during each month of 1991 .
FY91 NSLS XRAY RING;

AVERAOE FILLING CURRENT

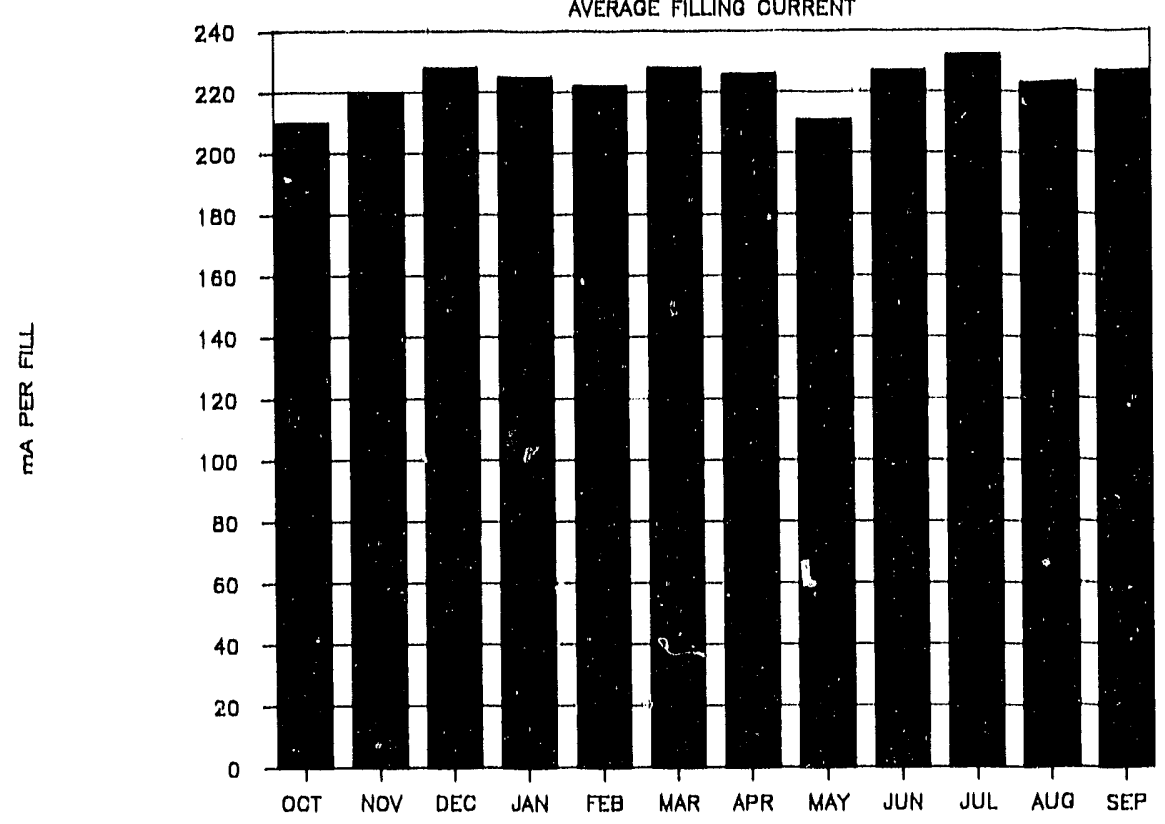

FY91 NSLS XRAY RING

AVERAGE 150 INA LIFETIME

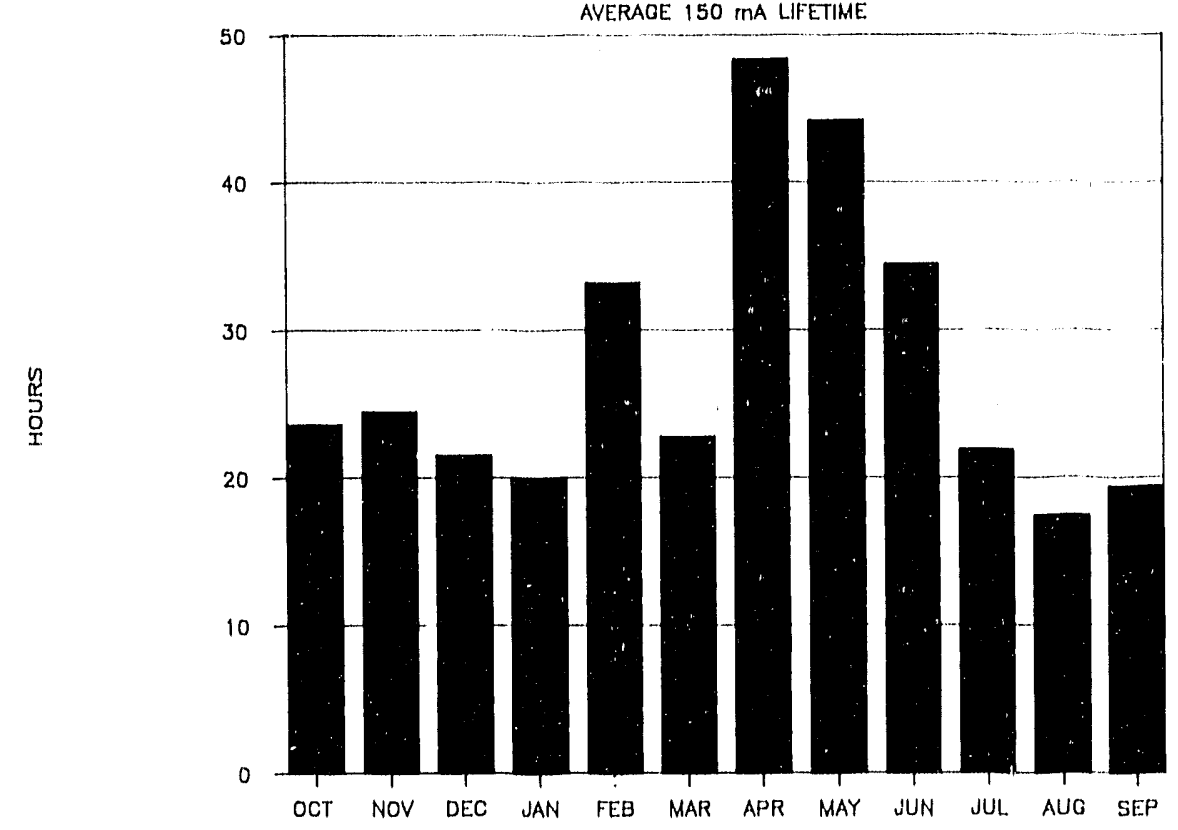

Fig. 4 The X-ray Ring exponentlal beam lifetime at $150 \mathrm{~mA}$ beam current averaged over each month during flecal year 1991. 


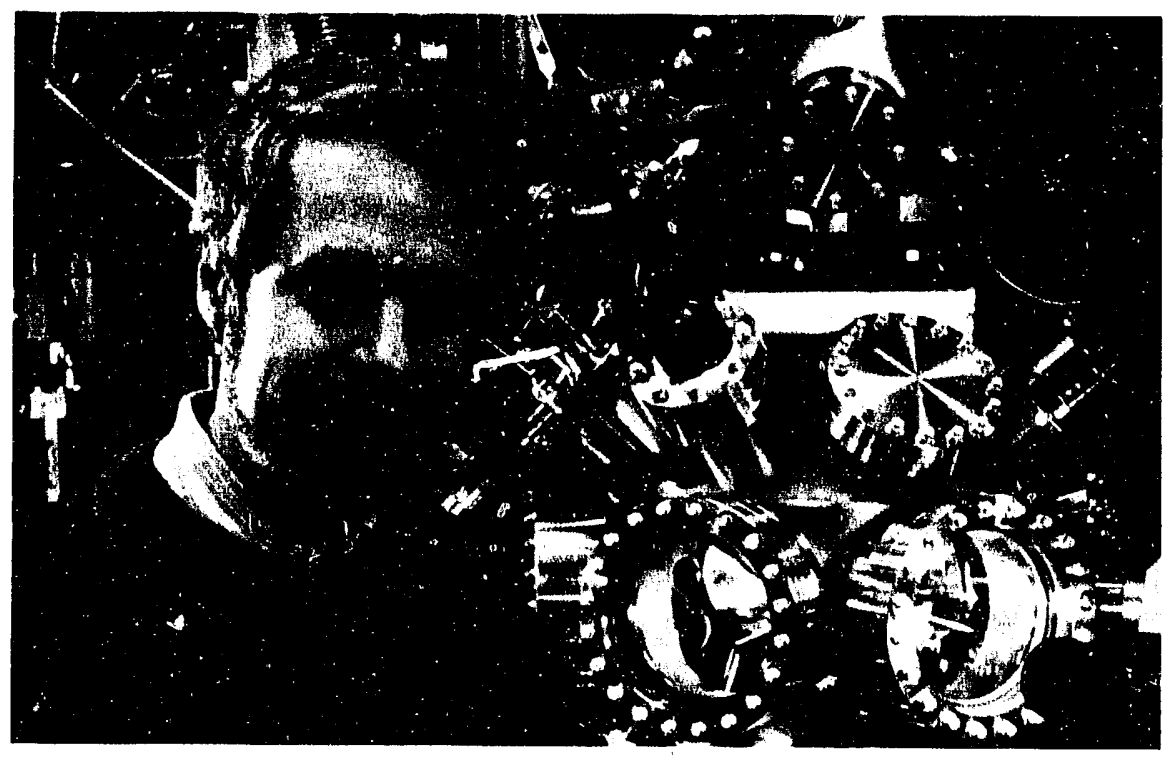

rastan

\section{X-RAY BEAMLINE OPERATIONS}

\section{Roger Klaffky \\ NSLS \\ $X$-ray Operations Manager}

During FY 1991 routine operations began on four more X-ray beamlines, bringing the total number of operational beamlines to 55 . These included X1B, X6B, $\mathrm{X} 13 \mathrm{~B}$, and X17B2 (Angiography). Construction and/or commissioning took piace on $\mathrm{X} 4 \mathrm{~A}, \mathrm{X} 6 \mathrm{~A}, \mathrm{X} 21$, $\mathrm{X} 28 \mathrm{~A}, \mathrm{X} 28 \mathrm{~B}$ and X28C. A count of NSLS Safety Approval Forms indicate that there were 872 different experiments performed, of duration several days to several months, representing a $14 \%$ increase over FY 1990. The breakdown of scientists participating on these experiments was $46 \%$ from universities, $21 \%$ from industry, $16 \%$ from BNL, $7 \%$ from other DOE laboratories, $6 \%$ from gov snment labs, and $4 \%$ from abroad.
There were a number of improvements in beamline capabilities this year. Installation of a watercooled safety shutter in the X1 front end allows the $\mathrm{X} 1$ local orbit feedback system to remain on when the safety shutter is closed. The X1A1 soft X-ray microscope line has obtained $50-75 \mu \mathrm{m}$ resolution luminescence images from fluorescence dyes such as are used to tag sites of specific biochemical activity in fluorescence microscopy. The X1A2 SPEM achieved better than $0.2 \mu \mathrm{m}$ resolution ESCA for which it received an R\&D 100 award. In its early operational phase, the X1B soft X-ray spectroscopy beamline demonstrated that it has unmatched intensity and resolution. A set of large d-spacing multilayer crystals were purchased for the X2B tomography monochromator to increase intensity. Low temperature $\left(12^{\circ} \mathrm{K}\right)$ capability was added on the X3B1 EXAFS beamline. Various additions were made to the X14A diffraction line: a beam position monitor, an aperture with vertical motion for characterizing the monochromator sagit- tal focussing, and a diffractometer table with tilt and rotation capabilities. Utilization of storage phosphor plates was also implemented. The X5 LEGS beamline completed its first full year of operation. A higher power, shorter wavelength $(300 \mu \mathrm{m})$ laser was installed to increase the $\gamma$-ray energy from 300 to $340 \mathrm{MeV}$. Liquid helium III and helium IV targets were put into operation as well as two new detectors - an array of 48 scintillator bars for neutrons and a large sodium iodide detector for gammas and high energy charged particles (pions, protons). On the X7A energy dispersive diffraction beamline, a water-cooled $\mathrm{Ge}(111)$ channel-cut monochromator was installed which doubles the intensity below the Ge $\mathrm{K}$ edge (11.1 $\mathrm{KeV}$. An NSLS-designed highstability horizontal focussing device with an assymetric-cut $\mathrm{Si}(220)$ crystal was installed for experiments using a high pressure chamber or for experiments using less than $200 \mu \mathrm{m}$ of horizontal beam. The focussing has led to an order of magnitude increase in flux on small samples. On the X7B crystallography beamline there were a number of additions: a linear $2 \mathrm{~cm}$ CCD detector; software for surface diffraction measurements, vertical scan capability for the sample table, and capability to scan the second monochromator crystal $\chi$ angle. A new CCD camera was installed on $\mathrm{X} 8 \mathrm{C}$ for protein crystallography experiments and the X8A beamline was fully characterized. A sagitallyfocussing $\mathrm{Si}(111)$ monochromator was put into operation on $\mathrm{X} 9 \mathrm{~A}$ and a beryllium window installed at the end of X9B to replace a 0.003 " aluminum window 
arrargement which was prone to developing leaks after exposure to white beam. A mirror feedback system was implemented on $\mathrm{X} 10 \mathrm{C}$ to improve beam stability which is vital for measurements on submillimeter crystals using a small focussed beam. An EXAFS microprobe consisting of a mirror/ pinhole arrangement and a raster scanned sample was used with $(25 \mu \mathrm{m}) \times(25 \mu \mathrm{m})$ resolution elements. Sagittal focussing was introduced on the X11A beamline, leading to a two-fold intensity increase at $11 \mathrm{keV}$ and a four-fold increase at $6 \mathrm{keV}$.

The entire data collection system on X12B was revamped to improve reliability and a $2 \mathrm{D}$ detector put into routine operation. On X14A a low temperature Displex unit was installed with a hemispherical beryllium dome for sampling full reciprocal space volume. The X15A monochromator housing was upgraded to allow operation with a helium atmosphere. A customized fluorescence detector was installed on X15B for EXAFS measurements.

On the X16A five circle surface $\mathrm{X}$-ray diffractometer, a faster, smoother gearless servomotor was installed to increase the $\alpha$ angular range to $45^{\circ}$, and a position sensitive detector was put into operation. Work continued on the conversion of $\mathrm{X} 16 \mathrm{C}$ to a monochromatic beamline. The $17 \mathrm{~B} 1$ (Materials Science) and X17B2 (Medical Research) lines ran in a full-time operational mode. The X17 superconducting wirgler cryogenic system was upgraded with the purchase of a new cryogenic compressor and helium transfer lines, rebuilding of the helium liq- uefier and the old compressor for use as a spare, and an upgrade to automatic valve controllers. (X17C)

On X18A the data collection system was converted to a PC-based GPIB interface system, with software coritrol of motors, counter, multi-channel analyzer PC board and temperature controller. A low temperature UHV surface chamber mounted on the X18A Huber diffractometer successfully operated in the spring. The X19A external monochromator drive system was installed, leading to marked improvement in perform. ance and reliability. A window valve system was designed which will provide for seiection of a standard beryllium window or a high throughout diamond window for lower energies. Design work was carried out on the white beamline X19C for the addition of a bent cylindrical mirror, a monochromator and a liquid surface spectrometer. On the X20 beam. line turbopumps were replaced by ion pumps to reduce noise, the computer systems were upgraded, and the X-ray beam vertical offset in the X20C monochromator was decreased to 1.5 inches to reach higher energies. Beamline safety interlocks were completed on X21 and a monochromator vacuum tank installed in preparation for tests of the monochromator cooling scheme. On X22B a new liquid metal cell was installed and steps were taken to improve the beam. line capabilities so as to change or scan energy to $10 \mathrm{keV}$ in the horizontal diffraction mode. A new $3.5^{\circ} \mathrm{K}$ Displex refrigerator was installed on X22C. On X23A2 the $\mathrm{Si}(220)$ monochromator crystal was replaced with a $\mathrm{Si}(311)$ crystal to improve resolution and harmonic rejection. Also, an active feedback table was installed to isolate the monochromator from vibration sources. The computer system was upgraded to a full online EXAFS system similar to that on X19A. A vertical beam position monitor was installed on $\mathrm{X} 24 \mathrm{~A}$, and the monochromator differential pumping and crystal mount were improved for rapid crystal changes. Sagittal focussing by the second Ge(220) monochromator crystal was implemented, reducing the amount of focussing required from the downstream toroidal mirror thereby extending the energy range. The beamline optics on the X25 wiggler were fully commissioned and a full experimental program commenced. On X26A a monochromator designed for two channel-cut $\mathrm{Si}(111)$ crystals was put into operation using a single $\mathrm{Si}(111)$ crystal for energy scans between four and fifteen $\mathrm{KeV}$. Construction of the $\mathrm{X} 28 \mathrm{C}$ diagnostic line for X-ray orbit position monitors was completed prior to taking first beam.

There were a number of changes on the X-ray floor. A mezzanine and elevated walkway were constructed at X5 to maintain an access route into the $\mathrm{X}$-ray Ring while allowing the construction of the $\mathrm{X} 4 \mathrm{C}$ and $\mathrm{X} 6 \mathrm{~A}$ hutches. $\mathrm{A}$ darkroom was constructed next to $\mathrm{X} 19 \mathrm{C}$. The area vacated by the NSLS Vacuum Group close to $\mathrm{X} 24$ was converted to a set-up space for staging of experiments on the X-ray and UV Rings. A new vent line was added outside the building for purging the $\mathrm{LN}_{2}$ transfer line prior to filling dewars. 
To improve communications between the NSLS and users an ad. ditional channel (CH.2) was added to the TV monitors for expanded messages on machine problems, a user pager system was put into operation to announce unexpected beam dumps and to indicate the return of beam after such dumps, and electronic mailing of the weekly user meeting minutes began. A Safety Approval Form data base was also implemented in the control room.

\begin{tabular}{|c|c|}
\hline \multicolumn{2}{|c|}{ X-Ray Storage Ring Parameters as of January 1992} \\
\hline Parameters & X-Ray Storage Ring \\
\hline Normal Operating Energy & $0.75-2.5 \mathrm{GeV}$ \\
\hline Maximum Operating Current & $0.25 \mathrm{amp}\left(10^{12} \mathrm{e}^{-}\right)$ \\
\hline Lifetime & -20 hours \\
\hline Circumference & 170.1 meters \\
\hline Number of Beam Ports of Dipoles & 30 \\
\hline Number of Inserition Devices & 5 \\
\hline Maximum Length of Insertion Devices & $<4.50$ meters \\
\hline$\lambda_{c}\left(\mathrm{E}_{\mathrm{c}}\right)$ at $1.22 \mathrm{~T}(\mathrm{~B})$ & $2.48 \mathrm{~A}(5 \mathrm{keV})$ \\
\hline$\lambda_{c}^{c}\left(E_{c}\right)$ at $5.0 \mathrm{~T}(\mathrm{~W})$ & $0.60 \mathrm{~A}(20.8 \mathrm{keV})$ \\
\hline$B(\rho)$ & 1.22 Tesla (6.875 meters) \\
\hline Electron Orbital Period & 567.7 nanoseconds \\
\hline Damping Times $(2.5 \mathrm{GeV})$ & $\tau_{x}=\tau_{y}=6 \mathrm{msec} ; t_{\varepsilon}=3 \mathrm{msec}$ \\
\hline Touschek $(2.5 \mathrm{GeV}, 0.25 \mathrm{~A})$ & 216 hrs $\left(v_{\mathrm{RF}}=700 \mathrm{kV}\right)$ \\
\hline Lattice Structure (Chasman-Green) & Separated Function, Quad Triplets \\
\hline Number of Superperiods & 8 \\
\hline Magnet Complement & $\begin{array}{l}16 \text { Bending ( } 2.7 \text { meters each) } \\
40 \text { Quadrupole }(0.45 \text { meters each }) \\
16 \text { Quadrupole }(0.80 \text { meters each }) \\
32 \text { Sextupole }(0.20 \text { meters each })\end{array}$ \\
\hline Nominal Tunes $v_{x}, v_{y}$ & $9.15,6.20$ \\
\hline Momentum Compaction & 0.0065 \\
\hline R.F. Frequency & $52.88 \mathrm{MHz}$ \\
\hline Radiated Power for Bending Magnets & $126 \mathrm{~kW} / 0.25 \mathrm{amp}$ of Beam \\
\hline R.F. Peak Voltage & $700 \mathrm{kV}$ \\
\hline Design R.F. Power & $300 \mathrm{~kW}$ \\
\hline$v_{\mathrm{s}}$ (Synchrotron tune) & 0.002 \\
\hline Natural Energy Spread $\left(\sigma_{\varepsilon} / E\right)$ & $8.2 \times 10^{-4}$ \\
\hline Natural Bunch Length $(2 \sigma)$ & $10.5 \mathrm{~cm}$ \\
\hline Number of RF Buckets & 30 \\
\hline Typical Bunch Mode & 25 . \\
\hline Horizontal Damped Emittance $\left(\varepsilon_{\mathrm{x}}\right)$ & $10^{-7}$ meter-radian \\
\hline Vertical Damped Emittance $\left(\varepsilon_{y}\right)^{\wedge}$ & $10^{-9}$ meter-radian \\
\hline Power per Horizontal milliradian, $0.25 \mathrm{~A}$ & 20 watts \\
\hline Typical Arc Source Size: $\sigma_{h}, \sigma_{v}$ & $\sim 0.35 \mathrm{~mm}, \sim 0.15 \mathrm{~mm}$ \\
\hline
\end{tabular}

Source of Data: NSLS Parameters, January 1983, compiled by A. van Steenbergen; updated values provided by Anne-Marie Fauchet and Norman Fewell (NSLS). 


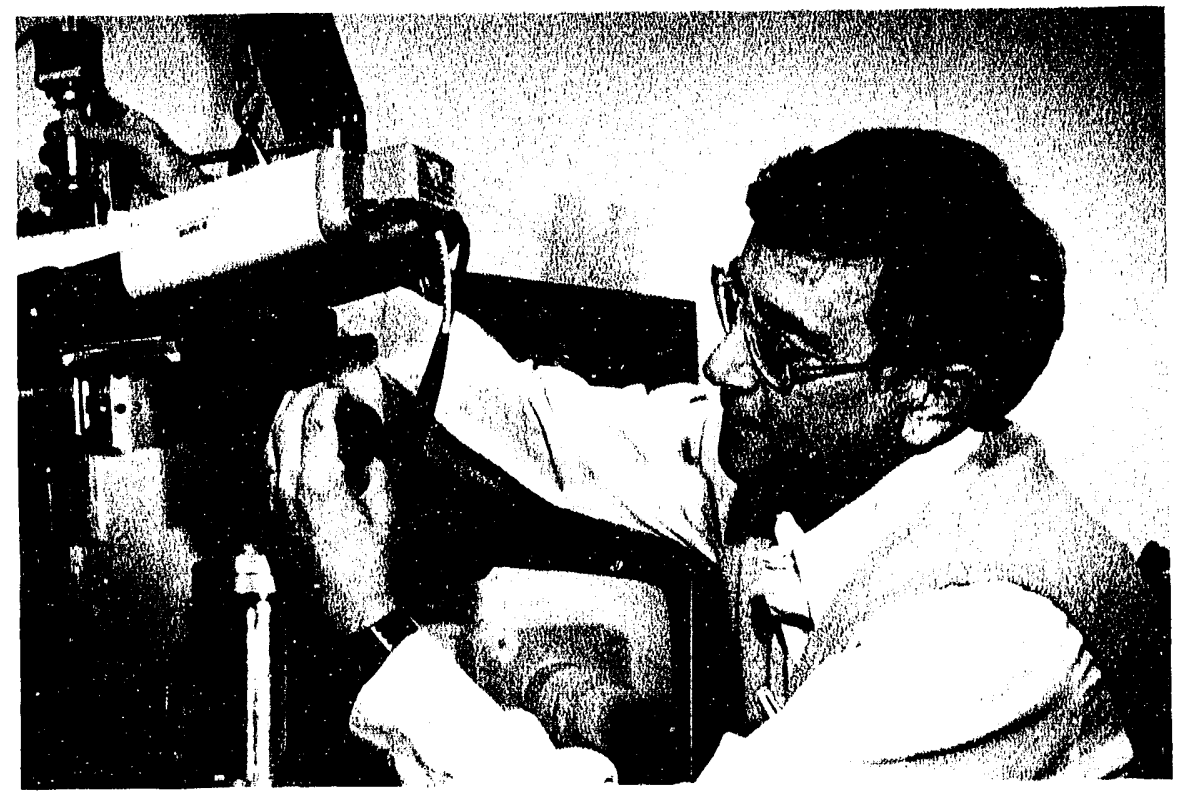

USER FACILITIES

\section{William Thomlinson NSLS Associate Chairman}

The NSLS has, for a number of years, had an aggressive policy towards providing the Users with as good an environment for doing research as possible. Of course, fiscal, personnel, and space constraints have forced some limitations and compromises. However, this past fiscal year has seen some major upgrades in the quality of life come to fruition. Perhaps the most obvious to any visitor to the NSLS is the User Administration Office (UA) which has been moved to a very convenient, central location. The location, coupled with an expansion of the space available to the Users for registration, has created a central information and reception area of which we are very proud. It is not just cosmetics which greet the User. Recently, the User database has been implemented in the UA Office which, for the first time, allows Users to register by direct input into computer terminals. The duplication of entries is a thing of the past, with all necessary forms now being printed from the computer. The database registration allows the NSLS to meet all the new DOE demands on foreign national registration and facility utilization statistics in an efficient manner.

In order to move the UA Office to its proper location, another major improvement in life style at the NSLS took place. The old library has been moved to a new location and converted into the beautiful Chasman-Green Memorial Library/User Lounge. It is located where the NSLS stock room used to be. The stockroom has been moved to a nearby, convenient location and continues to provide the excellent 24 hour/day service for which it has gained a lot of praise. The library/lounge is an excellent place to use to get away from the hustle and bustle of the experimental floor, to have a small meeting or discussion of new results, or to explore the most recent literature.

This year has seen a very welcome increase in the scientific staff dedicated to User support on NSLS beamlines. Dr. Lars Furenlid has taken charge of the X19A spectroscopy beamline and has worked closely with the existing PRT to upgrade the quality and quantity of beamtime available to users. Recently, the NSLS was pleased to bring on board Dr. Mei-Ling Shek to run the U14A General User photoemission spectroscopy line. She brings years of experience in the field to the program of upgrades and expansion of the U14A facility. The NSLS now has at least one scientist associated with each NSLS operated beamline.

Safety is now a part of the culture of the NSLS and the highly successful upgrade in the quality of the NSLS User Shop is a prime example. Mr. Gerry Van Derlaske has joined the Experimental Program Support Section as User Shop Manager and is available during all normal working hours to assist anyone in need of the shop, to answer questions, to keep our full complement of tools in proper working condition, and to help train those users needing shop experience. A written document of basic rules and safety orientation has been prepared and is being used as the minimum basis for qualification for access to the shop. Mr. Van Derlaske is in charge of the training and the documentation of qualified users. The safe, productive environment now 
found in Mr. Van Derlaske's Shop has resulted in improved safety and many positive comments from Users. The NSLS continues to put significant resources into the shop, knowing that quick turnaround of those many necessary "widgets" at any hour of the day or night is sometimes critical to success of an experiment. A new set of Shop User Rules has been established and already has resulted in a safer, cleaner Shop.

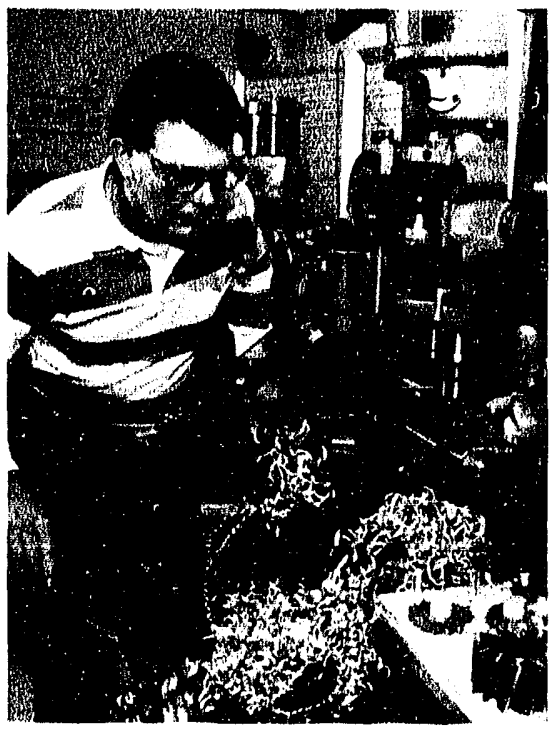

Gerry Van Derlaske, User Shop Manager

Effective interaction of the burgeoning NSLS User population with the NSLS Safety Personnel is critical to continuing our very high level of safety. In order to bring about closer communication between the Users and Safety Personnel most of the NSLS Safety Staff have now been concentrated in one central area adjacent to the experimental floor. Andrew Ackerman and Clem Auguste of the NSLS have joined John Aloi, Chris Weilandics, and Rudy Zantopp from the BNL S\&EP Division in offices near the NSLS Floor Manager. Also lo- cated in the same area are Randy Church, Supervisor of the Machine Operators and Operations Coordinators, and Steve Kemp, Maintenance Coordinator and Lead Operations Coordinator. Contiguous space has now been made available to the Coodinators so that they can continue to carry out technical projects when not on shift. Thus, Users can now go to one area of the NSLS to attend to any safety related issues or obtain assistance from the Floor Manager. The Floor Manager is now Mr. Richard Rothe who has replaced Mr. Mike Kelly.

This year saw the commissioning of the new communications chan. nel on the NSLS video system. Channel 2 has been a very successful means of getting information to the Users during times of trouble with the machines, scheduling, computers, etc. Since it is independent of the main system control computers, it has proven many times to be the only effective means of communication from the Control Room to the experimenters.
As most Users know by now, the new card reader access is in place. After many months of problems it seems to be working reasonably well. It has been beneficial to the NSLS in access control for contractors and security of the building. The minor problems which still exist should be ironed out very soon.

The new gas cylinder area and liquid nitrogen fill systems have been completed and provide a much safer, weather protected environment. The NSLS has targeted funds for expansion of the existing area for storage of liquid helium and nitrogen dewars, hazardous geses, and additional gas cylinders.

Life is still not all rosy at the NSLS, but initiatives are underway to solve many of the existing problems. Throughout this year, like most, there have been problems with communication between the NSLS and the BNL Plant Engineering Division. This has led to disruptions on the experimental floor. In order to solve

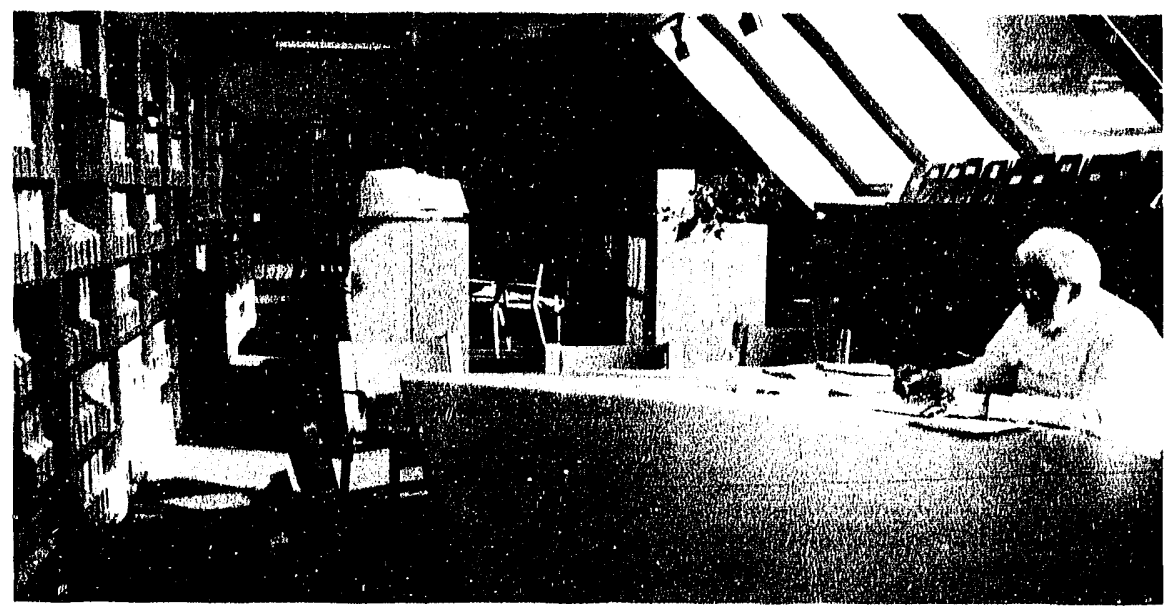

The Chasman-Green Memorial Library and User Lounge. 
this problem, the NSLS Management has been working with Plant Engineering on the means of notification of pending work and lines of responsibility. We are hopeful that the system we are now trying will lead to far fewer instances of work being started without proper notification of the affected persons.

Space continues to be a serious problem for the NSLS Commu- nity, both Users and Staff. As Denis McWhan discussed in his introduction to this report, two new inititatives for expansion of the present building is underway to add very badly needed space to the experimental floors. It is per. haps good to take a look at the space which the NSLS has made, and will make, available to the Users. The accompanying layout of the NSLS Experimental Floors shows the space available for ex- perimental programs as shaded areas. In addition, the User Shop in the basement of Building 535 and the office and lab space in Building 510 should be included. It is obvious that we are making every effort possible to provide working and living space to the User Community.

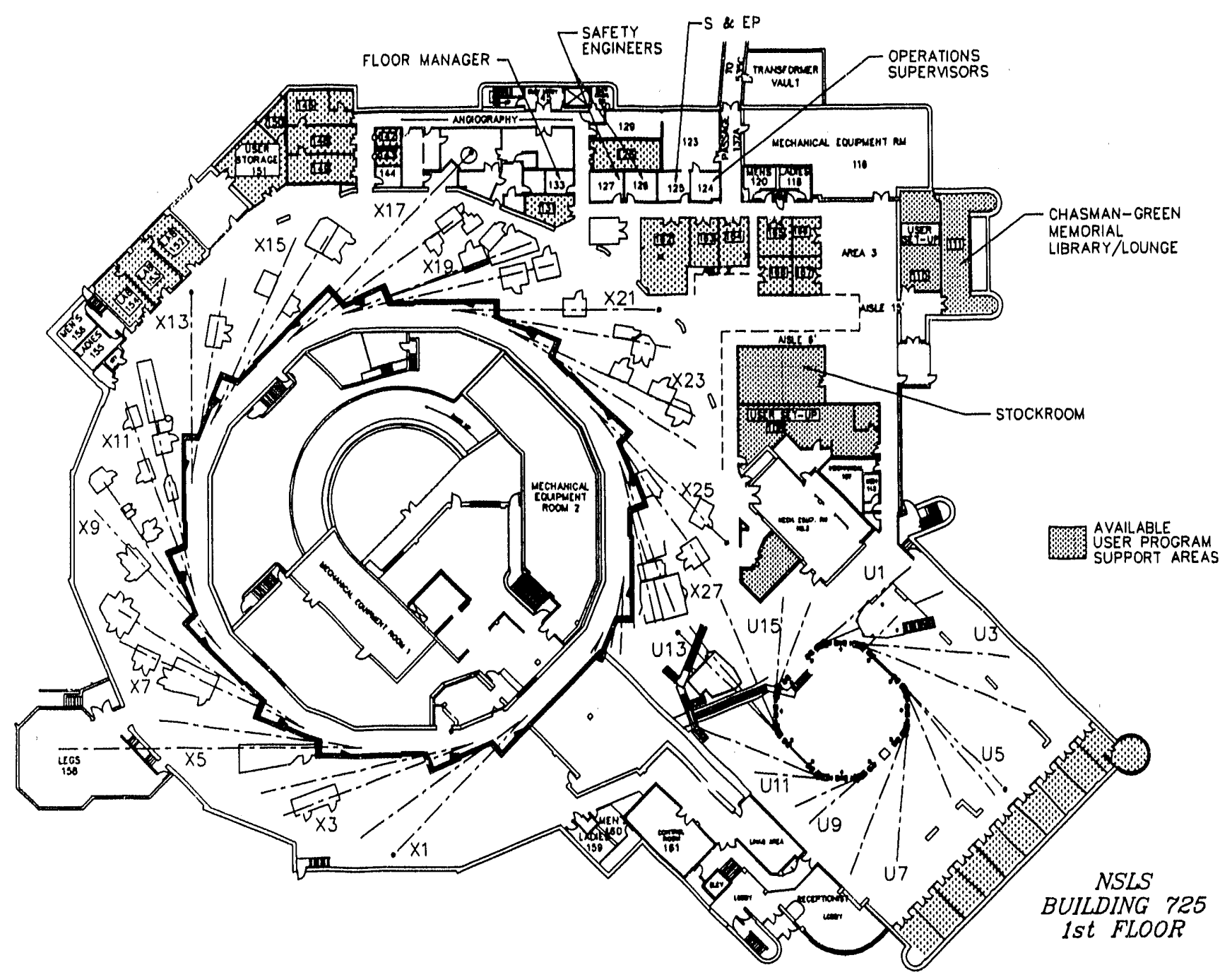

NSLS Experimental Floor Layout (shaded areas are spaces available for experimental programs). 

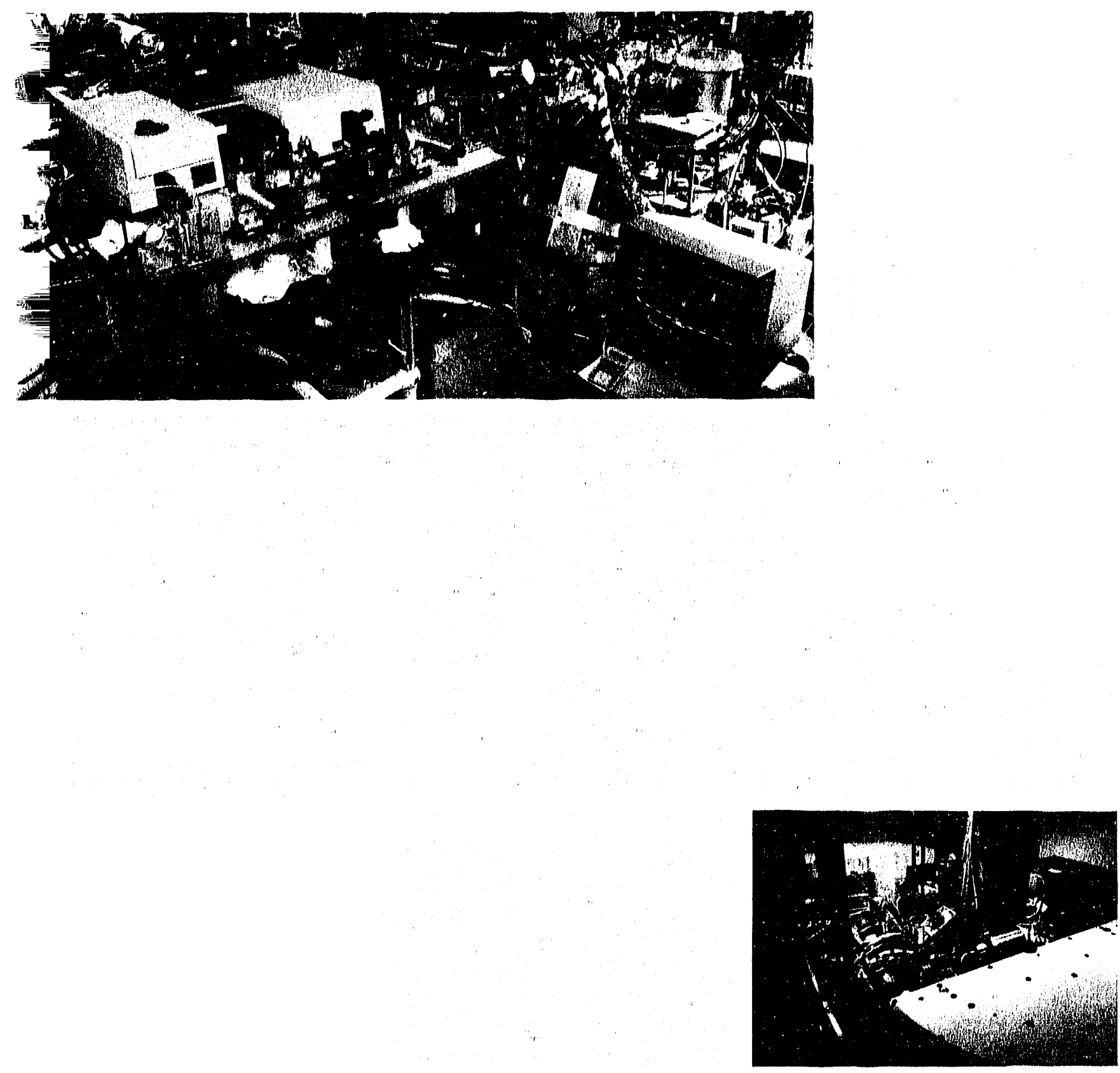


\section{SUPERCONDUCTING X-RAY LITHOGRAPHY SOURCE}

\section{Richard Heese}

NSLS

SXLS Project Head

The Phase I SXLS Ring has been running successfully for the last year, and has yielded a wealth of accelerator physics information on the performance of very com. pact synchrotrons. The ring's nominal design current of $500 \mathrm{~mA}$ at $200 \mathrm{MeV}$ is consistently being exceeded during studies runs. The peak current that has been stored is $1,200 \mathrm{~mA}$, far more than any other compact synchrotron in the world. The machine has proved to be extremely reliable, and the control system, a central data base type system using a graphics oriented control interface with the operator, has proven to be very capable and simple to learn and use. The major impediments to totally smooth operations are ion trapping effects in the circulating beam and beam instabilities. Efforts to understand and control these effects are ongoing.
A linear accelerator, which will serve as an injector for the superconducting storage ring, is being manufactured. This machine is a $200 \mathrm{MeV}$ high-current, short-pulse electron accelerator and will be able to fill the superconducting ring in about 15 seconds.

The superconducting dipole is continuing to be the subject of intensive study. It is a warm bore, iron-free magnet. The NSLS engineering staff has designed a novel support structure to allow extraction of synchrotron radiation from nine ports in each dipole while preventing distortion of the coils due to 200 tons of electromagnetic forces, while keeping the coils at cryogenic temperature.

Due to the strong curvature of the magnet coil structure, large values of higher harmonics, in addition to the required quadrupole and sextupole terms, are generated. This seriously reduces the dynamic aperture of the machine. Therefore, in addition to the basic coils, higher order correction coils are incorporated in the magnet along with trim coils for the quadrupole and sextupole components and vertical steering. Particle tracking with sophisticated codes, developed both at NSLS and elsewhere, confirm the effectiveness of these coils.

The detailed engineering design of the superconducting dipole mag. net by General Dynamics was finally completed. Some scheduling delays have occurred due to the complexities of the superconducting dipole. The first magnet is expected to be delivered in January 1993, and the second magnet in May 1993.

The Technology Transfer Program continued in 1991: five people from Grumman Aerospace Corporation were on site, working with us as part of this project, along with the team at General Dynamics involved in the design and construction of the superconducting dipole.

The scientific aspects of the project are proceeding very well. The continuing cost escalation and schedule delays of the superconducting dipole, however, are a cause for concern. Other options to manufacturing the magnets with cost containment and reasonable schedules are being examined. 


\begin{tabular}{|c|c|c|}
\hline \multicolumn{3}{|c|}{ SXLS Storage Ring Parameters as of January 1992} \\
\hline Parameters & SXLS Phase I & SXLS Phase II \\
\hline Normal Operating Energy & $0.2 \mathrm{GeV}$ & $0.696 \mathrm{GeV}$ \\
\hline Dipole Magnet Type & Electromagnetic & Superconducting, \\
\hline Maximum Operating Current & $\begin{array}{l}0.50 \text { amp }\left(8.8 \times 10^{10} \mathrm{e}^{-}\right) \\
(\text {measured) }\end{array}$ & $\begin{array}{l}0.5 \text { amp }\left(8.8 \times 10^{10} \mathrm{e}^{\prime}\right) \\
(\text { design })\end{array}$ \\
\hline Circumference & 8.503 meters & 8.503 meters \\
\hline Number of Beam Ports on Dipoles & 2 & 18 \\
\hline Number of Insertion Devices & 0 & 0 \\
\hline$\lambda_{c}\left(E_{c}\right)$ & $424 \mathrm{~A}(29.3 \mathrm{eV})$ & $10.0 \mathrm{~A}(1240 \mathrm{cV})$ \\
\hline$B(\rho)$ & 1.1 Tesla $(0.6037$ meters $)$ & 3.87 Tesla ( 0.6037 meters) \\
\hline Ficld Index, $n$ & 0.1759 & 0.1759 \\
\hline Electron Orbital Period & 28.3 nanoseconds & 28.3 nanoseconds \\
\hline Damping Partition Numbers & $\mathrm{J}_{\mathrm{x}}=0.53, \mathrm{~J}_{\mathrm{y}}=1.0, \mathrm{~J}_{\varepsilon}=2.47$ & $\mathrm{~J}_{\mathrm{x}}=0.53, \mathrm{~J}_{\mathrm{y}}=1.0, \mathrm{~J}_{\mathrm{E}}=2.47$ \\
\hline Damping Times & $\tau_{\tau_{\mathrm{c}} \cong 91 \mathrm{msec} ; \tau_{y} \cong 48 \mathrm{msec}} \cong 20$ & $\begin{array}{l}\tau_{x} \cong 2.16 \mathrm{msec} ; \tau_{y} \equiv 1.15 \mathrm{msec} ; \\
\tau_{\mathrm{c}} \cong 0.46 \mathrm{msec}\end{array}$ \\
\hline Uncorrected Chromaticity & $\xi_{x}{ }^{\varepsilon}=-0.49, \xi_{y}=-1.32$ & $\xi_{x}^{\varepsilon}=-0.49, \xi_{y}=-1.32$ \\
\hline Lattice Structure & Gradient FODO & Gradient FODO \\
\hline Number of Superperiods & 2 & 2 \\
\hline Magnet Complement & $\begin{array}{l}\text { SF Sextupole }(0.05 \text { meters }) \\
\text { QF Quadrupole }(0.155 \text { meters }) \\
\text { D1 Drift }(0.8965 \text { meters }) \\
\text { B Dipole }(1.896 \text { meters }) \\
\text { D1 Drift }(0.8965 \text { meters }) \\
\text { QF Quadrupole }(0.155 \text { meters }) \\
\text { SF Sextupole ( } 0.05 \text { meters) }\end{array}$ & $\begin{array}{l}\text { SF Sextupole }(0.05 \text { meters }) \\
\text { QF Quadrupole }(0.155 \text { meters }) \\
\text { D1 Drift }(0.8965 \text { meters }) \\
\text { Dipole }(1.896 \text { meters }) \\
\text { D1 Drift }(0.8965 \text { meters }) \\
\text { QF Quadrupole }(0.155 \text { meters }) \\
\text { SF Sextupole }(0.05 \text { meters })\end{array}$ \\
\hline Nominal Tunes $v_{x}, v_{y}$ & $1.415,0.415$ & $1.415,0.415$ \\
\hline Momentum Compaction & 0.32 & 0.32 \\
\hline R.F. Frequency & $211.54 \mathrm{MHz}$ & $211.54 \mathrm{MHz}$ \\
\hline Radiated Power & $0.234 \mathrm{~kW} / \mathrm{amp}$ of beam & $34.4 \mathrm{~kW} / \mathrm{amp}$ of beam \\
\hline \multicolumn{3}{|l|}{ Radiated power } \\
\hline R.F. Peak Voltage (typical) & $45 \mathrm{kV}$ & $250 \mathrm{kV}$ \\
\hline Design R.F. Power & $15 \mathrm{~kW}$ & $65 \mathrm{~kW}$ \\
\hline$f_{S}$ (Synchrotron Frequency) & $225 \mathrm{kHz}$ & $368 \mathrm{kHz}$ \\
\hline Natural Energy Spread $\left(\sigma_{\varepsilon} / E\right) * *$ & $2.0 \times 10^{-4}$ & $6.9 \times 10^{-4}$ \\
\hline Natural Bunch Length $(2 \sigma) * *$ & $1.6 \mathrm{~cm}$ & $3.8 \mathrm{~cm}$ \\
\hline Number of RF Buckets & 6 & 6 \\
\hline Typical Bunch Mode & 1,6 & 1,6 \\
\hline Horizontal Emittance $\left(\varepsilon_{\mathrm{x}}\right)$ & $5.92 \times 10^{-8}$ meter-radian & $7.17 \times 10^{-7}$ meter-radian \\
\hline Vertical Emittance $\left(\varepsilon_{\mathrm{y}}\right)^{\mathrm{X}}$ & $5.92 \times 10^{-9}$ meter-radian & $7.17 \times 10^{-8}$ meter-radian \\
\hline Source Size: $\sigma_{h}, \sigma_{v}$ & $>0.34 \mathrm{~mm},>0.2 \mathrm{~mm}$ & $0.75 \mathrm{~mm}, 0.75 \mathrm{~mm}$ \\
\hline \multicolumn{3}{|l|}{${ }^{* *}$ Current and RF voltage dependent. } \\
\hline rce of Data: J & & \\
\hline
\end{tabular}




\section{ACCELERATOR TEST FACILITY}

\section{Ilan Ben-Zvi \\ NSLS \\ ATF Project Head}

Free-Electron Lasers (FEL) are considered by many to be the fourth generation synchrotron light sources. The NSLS is carry. ing out an extensive $R \& D$ program on short wavelength FEL, leading towards a proposal for a UV FEL User Facility (see "Proposed UV-FEL User Facility at the NSLS", in this Section). The Accelerator Test Facility ${ }^{1}$ (ATF) is a linac-laser complex, capable of producing high brightness electron beams and high power laser pulses synchronized with the electrons. The ATF is the test bed for developing and testing the components needed for a successful UV-FEL. At the same time, the ATF is serving an advanced accelerator $R \& D$ program such as laser acceleration of electron beams and the interaction of intense laser pulses with high brightness electron beams.

The ATF is operated as a users' facility for accelerator physicists. The high brightness electron beam of the ATF, produced by a laser-photocathode RF gun $^{2}$, is already available for experiments. Two experiments already share this ultra high brightness 4.5 $\mathrm{MeV}, 100$ ampere beam. One is a Smith-Purcell radiation experiment by the BNL Advanced Accelerator Group in the Physics Department, the other is a
Cerenkov FEL experiment by a Dartmouth-Oxford team.

This gun generates its high current - low emittance beam by irra. diating a high quantum efficiency metal cathode in the presence of very high intensity RF electric fields. The generation and careful manipulation of such beams are an area of intense activity in accelera. tor physics, being the key to short wavelength FELs. The performance of the gun in the past year has been outstanding. The ATF team has measured nano-Coulomb beam bunches about 10 picoseconds long (producing a peak current of about 100 amperes) with a normalized rms emittance of $4 \pi \mathrm{mm}$ mrad.

The other key components of the BNL ATF are a $50 \mathrm{MeV}$ (up to 100 $\mathrm{MeV}$ in the future) electron linac, a 10 ps $10 \mathrm{~mJ}$ Nd:YAG laser, a 10 $\mathrm{GW} \mathrm{CO}_{2}$ laser system producing 100 ps pulses (10 ps soon). The lasers are synchronized to within one picosecond to the electron beam pulses. During 1991, the 50 $\mathrm{MeV}$ beamline leading from the linac to the experiment hall was constructed. Fifty $\mathrm{MeV}$ beams will be tested in the linac building in spring 1992 and the $50 \mathrm{MeV}$ experimental program will be started in the fall of 1992.

ATF experiments with a special interest for the NSLS short wavelength FEL programs are the RF gun development program, the visible wavelength FEL oscillator experiment, and the high-gain harmonic-generation experiment. The FEL experiments also include the development of novel superconducting undulators providing a high field and very low magnetic fiold error.

The RF gun program produces the now famous "BNL gun" model, a one and a half cell $\mathrm{p}$ mode $\mathrm{S}$ band gun, operating at a peak cathode electric field of $100 \mathrm{MV} / \mathrm{m}$. How. ever our program has gone beyond that. An extremely fruitful collab. oration has been established with Grumman Aerospace Corporation in which a potentially higher brightness, but most importantly very high duty factor gun has been developed and is now in an advanced production ${ }^{3}$. To carry out this program Grumman has invested a significant amount of their internal $R \& D$ funding. The gun will be tested at the ATF.

The visible FEL oscillator ${ }^{4}$ will employ a superconducting microundulator 68 period, $0.88 \mathrm{~cm}$ period length and 0.5 Tesla peak field at a gap of $0.44 \mathrm{~cm}$ ). Prototypes of this undulator were built (up to 7 periods long) and per. formed extremely well in super. conducting tests ${ }^{5}$. The field errors of the undulator were very low as constructed, making this perhaps the first undulator that will require no trimming after manufacture. The FEL experiment makes use of the short period of the undu. lator and of the low emittance beam generated by the RF photocathode gun to operate at $500 \mathrm{~nm}$ with a $50 \mathrm{MeV}$ electron beam. Emittance scales with energy so that at $250 \mathrm{MeV}$ a wavelength of $100 \mathrm{~nm}$ would be practical. This experiment is done in collaboration with MIT and Rocketdyne.

The high-gain, harmonicgeneration experiment ${ }^{6}$ is another 
facet of the technology-transfer collaboration with Grumman, which is providing the undulator for the experiment. This undulator is a derivative of the superconducting micro-undulator of the visible FEL oscillator. It uses the same principle of a continuous yoke but has parabolic pole faces for two axis focussing and four distinct regions: a prebunching undulator, a dispersive section, an exponential growth section and a tapered section. This experiment emulates the FEL physics of the User Facility UV FEL. The principle of high gain harmonic gen. eration will be tested and characterized in these experiments, as well as the superconducting undulator, the undulator diagnostics, error and alignment studies, and tapering control.

Other light source techniques are being pursued at the ATF. A final example is the coherent synchrotron emission experiment, in which the short, high charge ATF pulse will generate coherent and intense $\mathrm{mm}$ wave radiation in a bending magnet. This radiation will also be used as a pulse length diagnostic for the ATF.
References

1. I. Ben-Zvi, The BNL Accelerator Test Facility and Experimental Program. Proceedings of the 1991 Particle Accelerator Conference, San Francisco, May 6-9 1991.

2. K. Batchelor, I, Ben-Zvi, R. C. Fernow, J. Fischer, A. S. Fisher, J. Gallardo, G. Ingold, H. Kirk, L. Lin, R. Malone, K. McDonald, I. Pogorelsky, D. Russel, T, Srinivasan-Rao, J. T. Rogers, J, F. Sheehan, T. Tsang, J. Sheehan, S. Ulc, X. J. Wang, M. Woodle, J. Xie, and R. Zhang, Performance of the Brookhaven Photocathode RF Gun, Proceedings of the 1991 International Free-Electron Laser Conference, Santa $\mathrm{Fe}$ NM.

3. I. S. Lehrman, I, A. Birnbaum, S. Z. Fixler, R. L. Heuer, S. Siddiqi, I. Ben-Zvi, K. Batchelor, J. C. Gallardo, H. G. Kirk, T. Srinivasan-Rao, Design of a High Brightness, High Duty Factor Photo. cathode RF Gun, Proceedings of the 1991 International FreeElectron Laser Conference, Santa Fe NM.
4. K. Batchelor, I, Ben-Zvi, R, C. Fernow, A. S, Fisher, A. Friedman, J, Gallardo, G. Ingold, H. Kirk, S. Kramer, L. Lin, J. T. Rogers, J, F. Sheehan, A. van Steenbergen, M. Woodle, J. Xie, L. H. Yu and R. Zhang, Status of the Visible Free-Electron Laser at the Brookhaven Accelerator Test Facility, Proceedings of the 1991 International Free. Electron Laser Conference, Santa Fe NM.

5. I. Ben-Zvi, R. Fernow, J, Gallardo, G. Ingold, W. Sampson, M. Woodle, Performance of a Superferric, High Field Subcentimeter Undulator, Proceedings of the 1991 International FreeElectron Laser Conference, Santa Fe NM.

6. I. Ben-Zvi, A. Friedman, C. M. Hung, G. Ingold, S. Krinsky, L. $H$. Yu, I. Lehrman and D. Weissenburger, Design of a Harmonic Generation FEL Experiment at BNL, Proceedings of the 1991 International Free-Electron Laser Conference, Santa Fe NM. 


\section{PROPOSED UV-FEL FACILITY AT THE NSLS}

\section{Ilan Ben.Zvi \\ NSLS}

UV-FEL Project Head

Sam Krinsky

NSLS

Deputy Chairman

\section{Li-Hua Yu \\ NSLS}

The NSLS is designing an accelerator based UV/VUV radiation source ${ }^{1,2}$ that will provide picosecond and sub-picosecond pulses of coherent ultraviolet radiation for wavelengths from 300 to 75 $\mathrm{nm}$. Pulse width will be variable from about $7 \mathrm{ps}$ to under $200 \mathrm{ps,}$ with repetition rates as high as 10 $\mathrm{kHz}$, single pulse energies $>1 \mathrm{~mJ}$ and therefore peak pulse power
$>200 \mathrm{MW}$ and average beam power $>10 \mathrm{~W}$ in a $10^{-4}$ bandwidth. The facility will be capable of "pump-probe" experiments utilizing the FEL radiation with 1) syn. chronized auxiliary lasers, 2) a second, independently tunable FEL beam, or 3) broad spectrum, highintensity $x$-rays from the adjacent National Synchrotron Light Source, The UV-FEL will make possible new avenues of inquiry in time resolved studies of diverse fields including chemical, surface and solid state physics, blology, and material science.

The UV-FEL beam is generated by a high duty factor laser photo. cathode RF gun, accelerated by a recirculating superconducting linear accelerator then separated into two pulse streams feeding two undulators. A drawing of the facility layout is shown below. The radiation is generated by "conventional" tunable Tisapphire lasers, multiplied by conventional techniques to the visible or near UV. This radiation is then injected into the bunching section of the undulator, which is resonant at the injected radia. tion's frequency. The energy modulated electron beam is bunched magnetically and fed into the next undulator section, now resonant at a VUV harmonic of the seed radiation. The radiation is now am. plified, first in an exponential section, then in a tapered undula. tor section and transferred to the users.

The wavelength is changed by tuning the seed laser (and varying the energy of the electron beam by the energy modulator cavities to maintain the FEL resonance condition). Thus fast tuning of as much as $30 \%$ is possible at each undulator. The two undulators will be staggered in wavelength, to provide a tuning range of $60 \%$ without a change in the linac's energy. The energy modulating

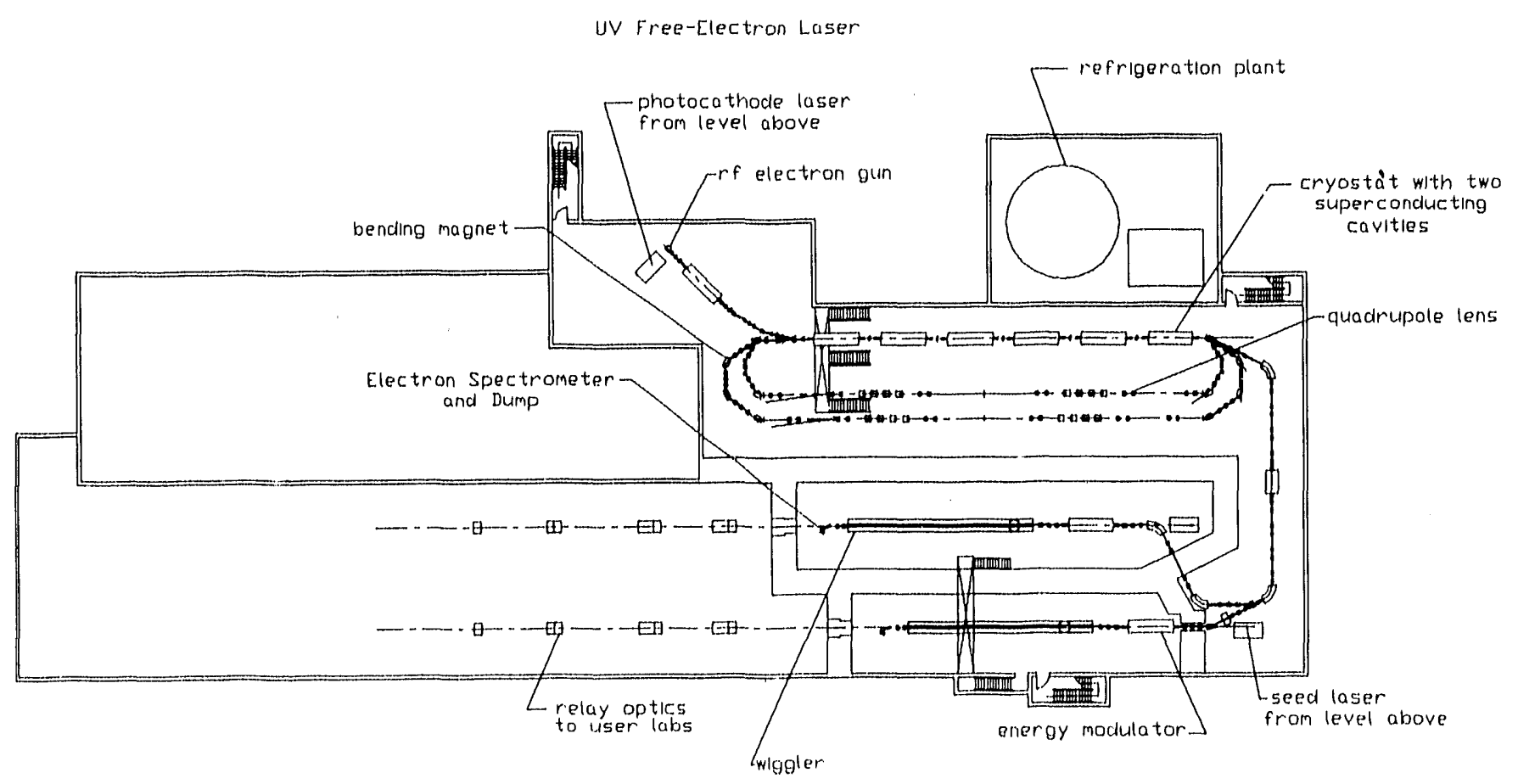


cavities come in slightly detuned pairs, so that alternate pulses in each of the undulators are inde. pendently tunable. Thus, up to four independent experiments may operate at one time, each with independent control of the wave. length and pulse duration.

To generate short wavelength coherent radiation one must gen. erate, transport, and accelerate an extremely low emittance electron beam without diluting it. The electron beam of the UV-FEL will be generated by a laserphotocathode RF gun of the type that has been developed at the Accelerator Test Facility ${ }^{3}$. To provide the high repetition rate that is needed for the User Facility, a high duty factor $\mathrm{RF}$ gun has been designed ${ }^{4}$ in collaboration with Grumman Aerospace Corporation. This gun will operate at a repetition rate of 5 $\mathrm{kHz}$ (macropulses) and deliver two pico-second pulses of between one and three nanoCoulombs per pulse at a variable multi-nanosecond separation in each macropulse. Thus the total repetition rate will be $10 \mathrm{kHz}$. Extensive simulations of the gun's performance lead us to anticipate a normalized $\mathrm{rms}$ emittance of about $4 \pi \mathrm{mm}$ mrad, a value that is similar to what has been measured at the $\mathrm{ATF}^{3}$.

At the energies required for a UV-FEL, the best combination of peak current, stability, energy spread and time structure is available from a superconducting linac at $500 \mathrm{MHz}$. With such a machine it is possible to run either short, sub-picosecond bunches or long, a few tens of picosecond bunches with little degradation of energy spread due to wake fields or funda. mental mode curvature. At 500 $\mathrm{MHz}$, it is possible to operate at $4.5 \mathrm{~K}$, simplifying the cryogenic system. Superconducting linacs are available commercially from a number of manufacturers. To re. duce the cost of the linac a recircu. lation scheme with about three passes through the linac will be used. This arrangement has additional advantages, such as the possibility of extracting bunches at various energi ss destined for a number of FELs operating at various wavelengths, as well as the op. tion to do bunch compression at an intermediate energy.

A $750 \mathrm{MHz}$ superconducting cavity modulates the energy of the accelerator beam. This choice of frequency allows us to accelerate one micro-pulse and decelerate the other, as long as their pulse separation is a multiple of $4 \mathrm{~ns}$. These micropulses are then separated by converting the energy difference into a trajectory modification that sends each micropulse into another undulator. Thus the radiation pulses generated in each undulator can be produced with a controlled small delay, from coincidence to many nanoseconds apart. This feature makes it pos. sible to do FEL on FEL pumpprobe experiments.

Unlike FEL oscillators requiring cavities, our FEL amplifier approach $^{6}$ eliminates the resonator mirrors which are a difficult aspect of oscillator FEL designs. The laser amplifier scheme proposed here will provide high peak power VUV radiation with the mode structure, bandwidth and frequency stability of an input seed laser.
Wavelength tuning will be done through energy modulation rather than through undulator gap change. (Naturally the seed lasers will tune in synchronism.) This final energy modulation will be done by special purpose acceler. ating cavities placed just before each of the two undulators, thus necessitating no transport ele. ment adjustments. This method offers the following advantages:

1. Simpler undulator design;

2. Undulator operation at its highest $\mathrm{K}$;

3. Much faster wavelength tun. ing, limited in principle only by the seed laser tuning speed.

The undulator design will be based on a superconducting undulator ${ }^{B}$ that has been developed at the ATF for a visible FEL oscillator experiment. This undulator has a high axial magnetic field, high precision and low cost per unit length.

The electron bunches in each undulator come at a repetition rate of $5 \mathrm{kHz}$. The energy modulation for wavelength tuning will be done by two cavities in series, one at the exact linac frequency and the other detuned by $2.5 \mathrm{kHz}$. The beating of the two frequencies will then create a differential energy modulation, every other pulse in the undulator having an indepen. dent energy setting. By switching between two seed lasers, one for each $2.5 \mathrm{kHz}$ stream of electrons, we create two independently tun. able FELs operating in the same undulator. The UV radiation pulses will be separated by rotating mirrors and sent to user's stations. Therefore the UV-FEL User Facility will serve four concurrent 
users, independently tuning their wavelength and pulse length.

The FEL output in $10^{-4}$ band. width is $1 \mathrm{~mJ}$ per pulse, resulting in an average power of 5 watts per undulator in two undulaiors. The availability of radiation with these characteristics would open up new opportunities in photochemistry, biology, material science and physics.

\section{References}

1. I. Ben-Zvi, L, F, Di Mauro, S. Krinsky, M. G. White and L. H. $\mathrm{Yu}$, Proposed UV-FEL User Facility at BNL, Nucl. Instr. and Meth. A304, 181 (1991).

2. I. Ben-Zvi, L. F. Di Mauro, S. Krinsky, M. G. White, L. H. $\mathrm{Yu}, \mathrm{K}$. Batchelor, A. Friedman, A. S. Fisher, H. Halama, G. Ingold, E. D. Johnson, S.
Kramer, J, T, Rogers, L. Solomon, J. Wachtel and $\mathrm{X}$. Zhang, Proposed UV.FEL User Facillty at BNL, Proceed. Ings of the 1991 International Freo-Electron Laser Confer. ence, Santa Fe NM.

3. K, Batchelor, I. Ben-Zvi, R, C. Fernow, J, Fischer, A, S. Fisher, J, Gallardo, G. Ingcld, H. Kirk, L. Lin, R. Malone, K. McDonald, I. Pogorelsky, D. Russel, T. Srinivasan-Rao, J. T. Rogers, J, F, Sheehan, T. Tsang, J. Sheehan, S. Ulc, X. J. Wang, M. Woodle, J, Xle, and R. Zhang, Performance of the Brookhaven Photocathode RF Gun, Proceedings of the 1991 International Free-Electron Laser Conference, Santa $\mathrm{Fe}$ NM.

4. I. S. Lehrman, I, A. Birnbaum, S. Z. Fixler, R. L. Heuer, S.
Siddiqi, I, Ben-Zvi, K. Batchelor, J. C. Gallardo, H, G. Kirk, T. Srinivasan-Rao, Design of a High Brightness, High Duty Factor Photo. cathode RF Gun, Proceedings of the 1991 International FreeElectron Laser Conference, Santa $\mathrm{Fe}$ NM.

5. L. H, Yu, Generation of Intense UV Radiation by Subharmoni. cally Seeded Single-pass FreeElectron Lasers. Phys. Rev A44, 5178 (1991).

6. I. Ben-Zvi, R, Fernow, J, Gallardo, G. Ingold, W. Sampson, M. Woodle, Per. formance of a Superferric, High Field Subcentimeter Undulator, Proceedings of the 1991 International Free. Electron Laser Conference, Santa Fe NM. 


\section{GLOBAL ORBIT FEEDBACK SYSTEMS}

\section{O. V. Singh \\ NSLS}

\section{H. Yu}

NSLS

\section{S. Krinsky \\ NSLS \\ Deputy Chairman}

Stability of the electron orbit is essential for the utilization of the NSLS low emittance storage rings as high brightness radiation sururces. For an individual user, ine required orbit stability can be achieved by a local bump feedback system. However, to install local bump feedback systems for every beamline is both costly and im. practical. The coupling between different local bumps may introduce instability, and there may not be enough space for the large number of trim coils required. Therefore, our approach to achiev. ing the necessary orbit stability has been the development and in. stal'ation of global orbit feedback systems ${ }^{1-3}$ based upon harmonic analysis of the orbit movements and the correction magnetic fields. A harmonic feedback system corrects the Fourier compnnents of th $\geqslant$ orbit nearest to the betatron tune. The Fourier analysis is done by a simple linear analog network. The input voltages are propor. tional to the orbit displacements at the detectors, and in real time the output voltages are propor. tional to the Fourier harmonic co- efficients. The fee'ioack does not force the displacement to zero at the detectors, but forces the coefficients of a few harmonics nearest the betatron tune to vanish.

The first development of the harmonic feedback system was a prototype system to correct the vertical orbit on the VUV Ring. This system uses four position monitors and four correctors, and has been successfully used in operations since 1989. Based upon the experience with the VUV Ring, feedback systems have been developed and installed during the last year on the X-ray Ring. Compared to the VUV Ring system, the new X-ray Ring systems eliminate three harmonic components of the crbit distortion instead of only one. In the low frequency circuits of the new systems, MDAC's (Multiplying Digital to Analng Convertors) were used to replace the potentiometers in the Fourier analysis networks. This makes the system more accurate and easier to install than the VUV Ring system. The vertical feedback has been successfully in operation on the X-ray Ring since February 1991 and the horizontal system since September 1991, providing a dramatic improvement in orbit stability of more than a factor of five at all source locations (see Figures 1 and 2). During installation a methed was developed to decouple seve."al local feedback sysiems and op rate them simultaneously with th ? global system. The global syst?m not only greatly reduces the orbit movement but it also significantly reduces the required feedback correction strengths of the local feedback systems.

A horizontal glnbal feedback system is scheduled to be installed in the VUV Ring by Spring 1992. followed by the installation of a new vertical system.

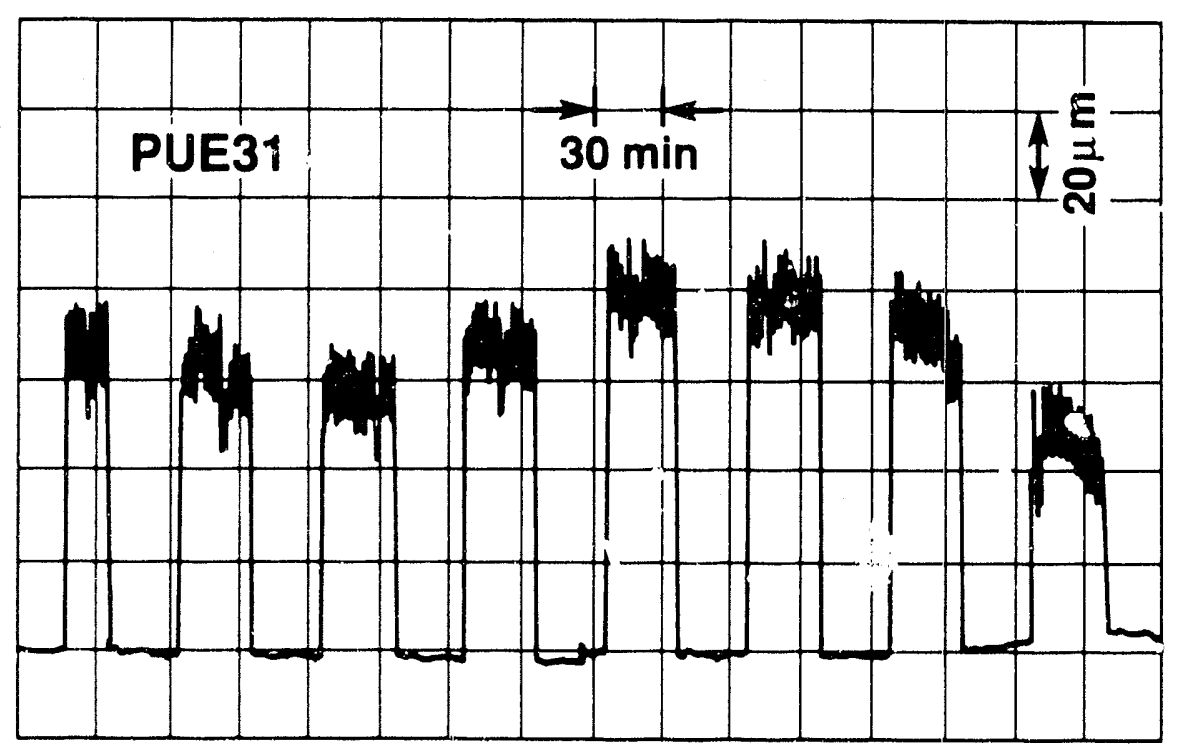

Fig. 1. Long.term and sho: 2 term vertical beam position movement at PUE 31 on the X-ray Ring when the glnbal feedback was turned on and off every $30 \mathrm{~min}$. durirg an $8 \mathrm{hr}$. machine study period. 


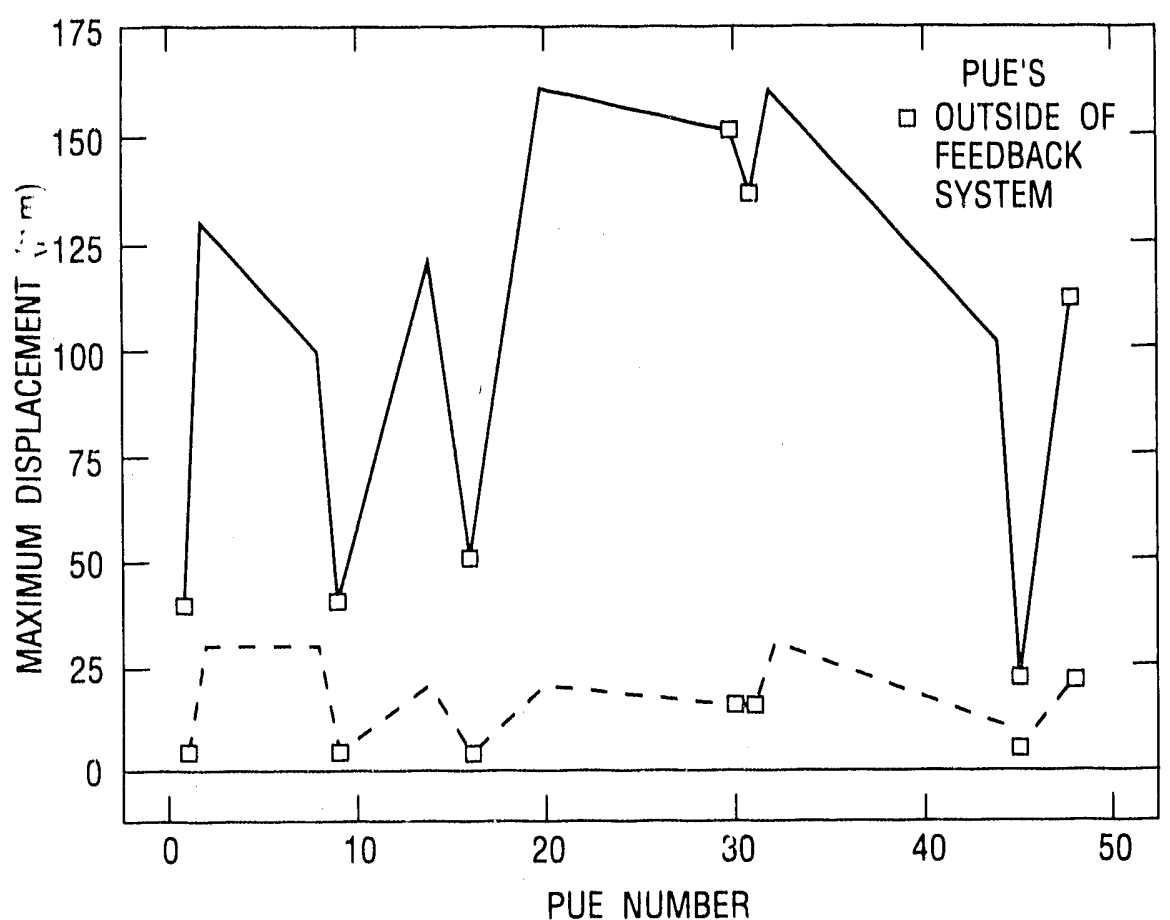

Fig. 2. Global improvement of orbit stability in the X-ray Ring upon closing the feedback loop. Solid curve represents orbit variation with feedback off and dashed curve with feedback on.
References

1. L. H. Yu, E. Bozoki, J. Galayda, , Krinsky, G. Vignola, Real time har. monic closed orbit correction schemes, Nucl. Instrum. Methods A284, 268 (1988).

2. L. H. Yu, R. Bisardi, J. Bittner, E. Bozoki, J. Galayda, S. Krinsky, R. Nawrocky, $O$. Singh, G. Vignola, Real time closed orbit correction system, Proc. 1989 Particle Accelerator Conf., Chicago, p. 1792.

3. L. H. Yu, R. Biscardi, J. Bittner, A.M. Fauchet, S. Krinsky, R. J. Nawrocky, J. Rothman, O. V. Singh, K. M. Yang, Real time global orbit feedback system for NSLS XRay Ring, Proc. 1991 Particle Accelerator Conf., San Francisco, p. 2542.

Effort has gone into improvements which are aimed at providing increased operational reliability of the NSLS facility. Work in this area includes a beamline status system which will provide the operations staff with the information to monitor the beamline front ends, vacuum, and water flow status. This information will help the operations staff determine and prevent the causes of beam dumps. Other work in this area included the conversion of some older multibus microcomputers to VME based systems. These systems included the booster and the single bunch micros. 

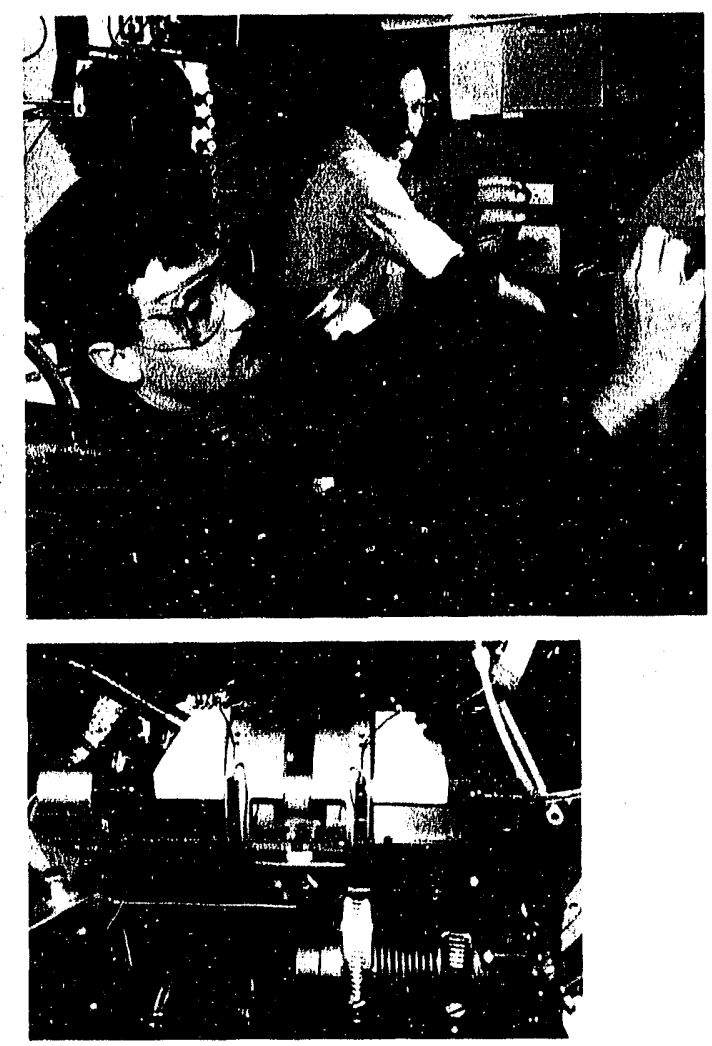


\section{Beamline U3A}

Los Alamos National Laboratory beamline U3A was converted from a white light line to a high throughput, low resolution line. U3A was formerly used with only a mirror and filter to provide a time resolved calibration of a $\mathrm{GaAs}(\mathrm{Fe})$ $\mathrm{x}$-ray calorimeter. We were able to track the change in bunch length with time after a VUV Ring fill, but our detector response calibration was unsatisfactory without beam monochromatization. In 1991 we completed a new monochromator, added a new detector calibration end station, added a water cooled filter holder, and completed modifications to the shielding required by the beamline review committee. All hardware additions were completed and installed on the beamline. The monochromator was internally aligned. The converted U3A is ready for its commissioning period. It has vacuum isolation from the ring through an efficient differential pumping system. This permits the monochromator to operate at $1 \times 10^{-6}$ torr with motors, encoders, and moving parts inside the chamber. Monochromator motions and readouts are controlled by a computer. The exit is fixed while the energy is tuned over a range from IR to $2 \mathrm{keV}$ by chosing among a selection of four pairs of dispersing elements, including crystals, multilayers, gratings, and mirrors. Resolution from the crystals and gratings can be high at the expense of flux, or vice versa. Most applications planned for U3A will use the high flux, low resolution mode. It is primarily a radiometric line, but will be made available to any qualified user who finds it suited to his scientific needs. R. L. Blake, B. Davis, R. G. Hockaday, and D. Holmberg (LANL).

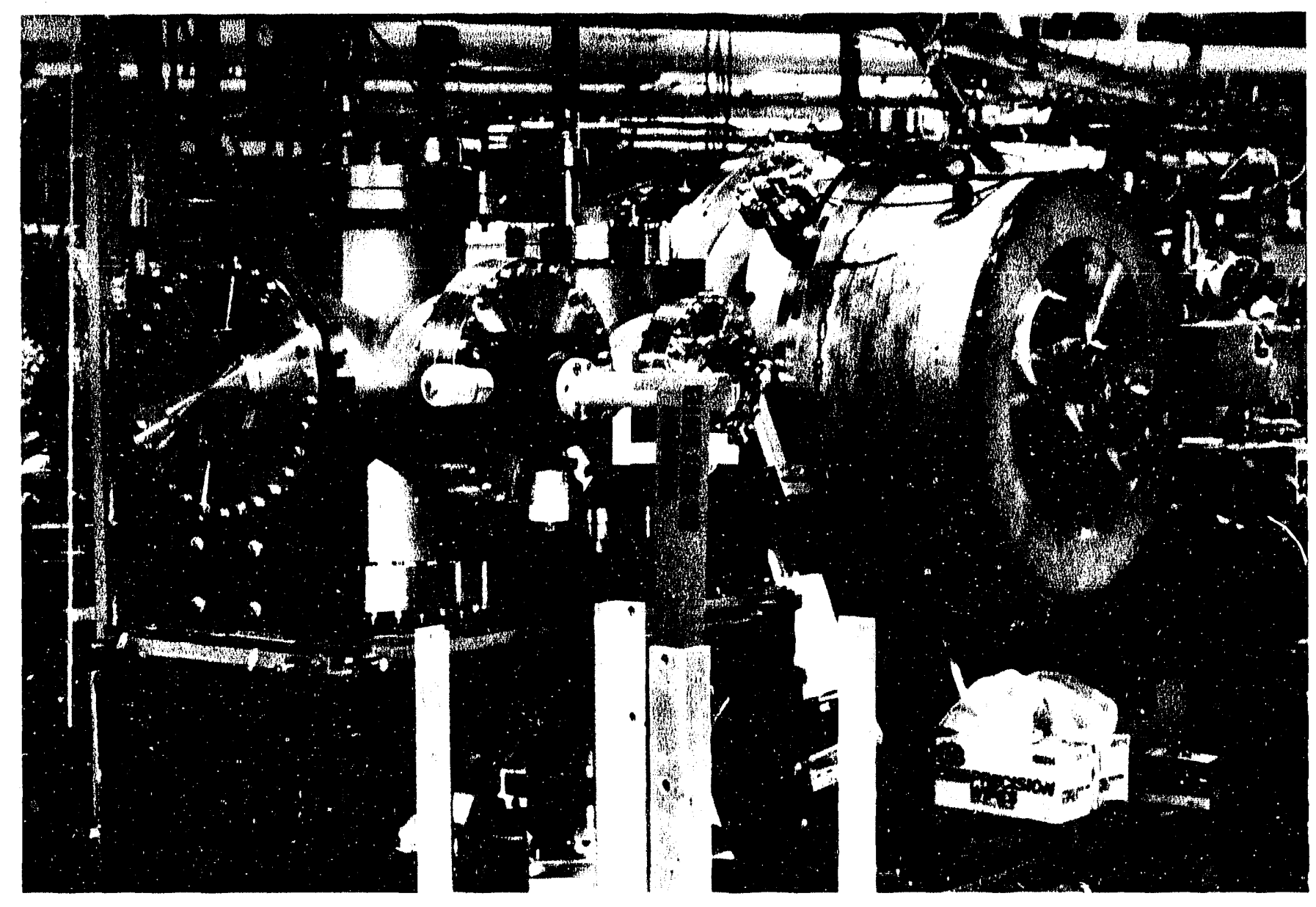

The above figure shows a view of the U3A beamline during re-assembly. The large chamber at right-center houses the new monochromator. Barely discernable to its right is some beamline shielding around the differential pumping station. Two of three calibration chambers are located left (downstream) of the monochromator. The converted beam line is now in the commissioning phase. 


\section{Beamline U4IR}

The high level of activity at the U41R Infrared beamline on the VUV storage ring is clear from the cluster of instruments surrounding the exit port. These include three Nicolet interferometers covering the wavelength region from 1 micron to $1 \mathrm{~mm}$. In the foreground is the IR microscope used for high pressure diamond anvil cell studies. To the right is the ultrahigh vacuum surface science chamber used for studying vibrations at metal surfaces. In the center rear, in* corporated into the spectrometer, is the chamber used for the high $\mathrm{T}_{\mathrm{c}}$ and pump-probe studies. For these latter experiments a laser light from U10 is brought via a fiber optic cable. The synchrotron beam can be quickly switched between any of the above instruments. C. Hirschmugl and G. Williams (NSLS).

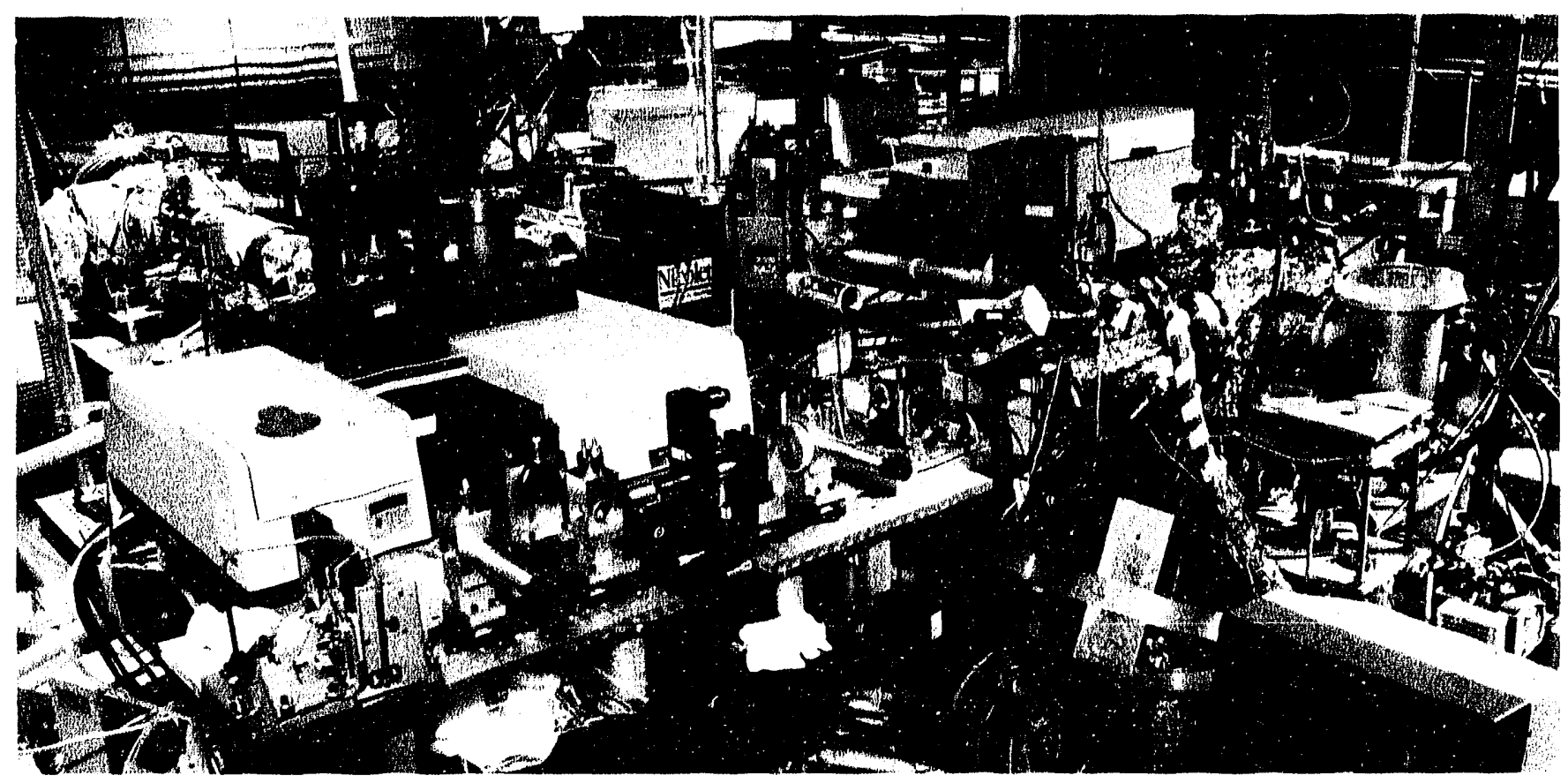

\section{Beamline U16B}

\section{Modification and Installation of Cornell End Station}

The current measurement chamber was significantly improved by the addition of a second chamber designed specifically for the insertion, modification and characterization of samples prior to transfer into the main measurement chamber. Operation of the modified new end-station in the final stages was evaluated on the beamline using a silicon, single crystal target. Due to problems associated with stored beam operating characteristics and instrumentational problems, the characterization studies of $\mathrm{CoSi}_{2}$ films and overlayers had to be rescheduled.

The modified end-station is $\oplus$ xpected to significantly enhance the output and quality of future in-situ film and catalyst studies on the beamline. T. N. Rhodin, R. P. Merrill, S. Woronick, and P. Ciszek (Cornell U.).

\section{Design of High Sensitivity Soft X-ray Fluoreecence Detector}

A high sensitivity x-ray fluorescence solid state detector, obtained from Canberra Instruments, was experimentally evaluated for the quantitative detection of very small quantities of low-Z elements (nitrogen, carbon and oxygen) normally present in powders and films using near edge adsorption spectroscopy. An instrument of modified design was designed and will be installed based on this work. It is expected that the application of this new instrument will substantially enhance measurement of NEXAFS and EXAFS spectra on nonconducting samples. T. N. Rhodin, R. P. Merrill, and S. Woronick (Cornell U.). 


\section{Beamline X5}

The target and detector stand for LEGS experiments on hydrogen and helium. The liquid target is enclosed in the transparent cylinder at the center of the assembly. Above the target is an array of forty-eight plastic scintillator bars to detect and identify neutrons and protons. Below the target are twenty-four plastic and $\mathrm{CaF}_{2}$ scintillator phoswiches for detecting protons and pions. The entire apparatus, including target cryogenic systems, is mounted on rails so that it can easily be moved into and out of the yatuma-ray beam, to allow other experiments to proceed while this apparatus is being modified and tested. It had $\mathcal{L}_{\mathrm{e}} \mathrm{n}$ in use for experiments during the past year.

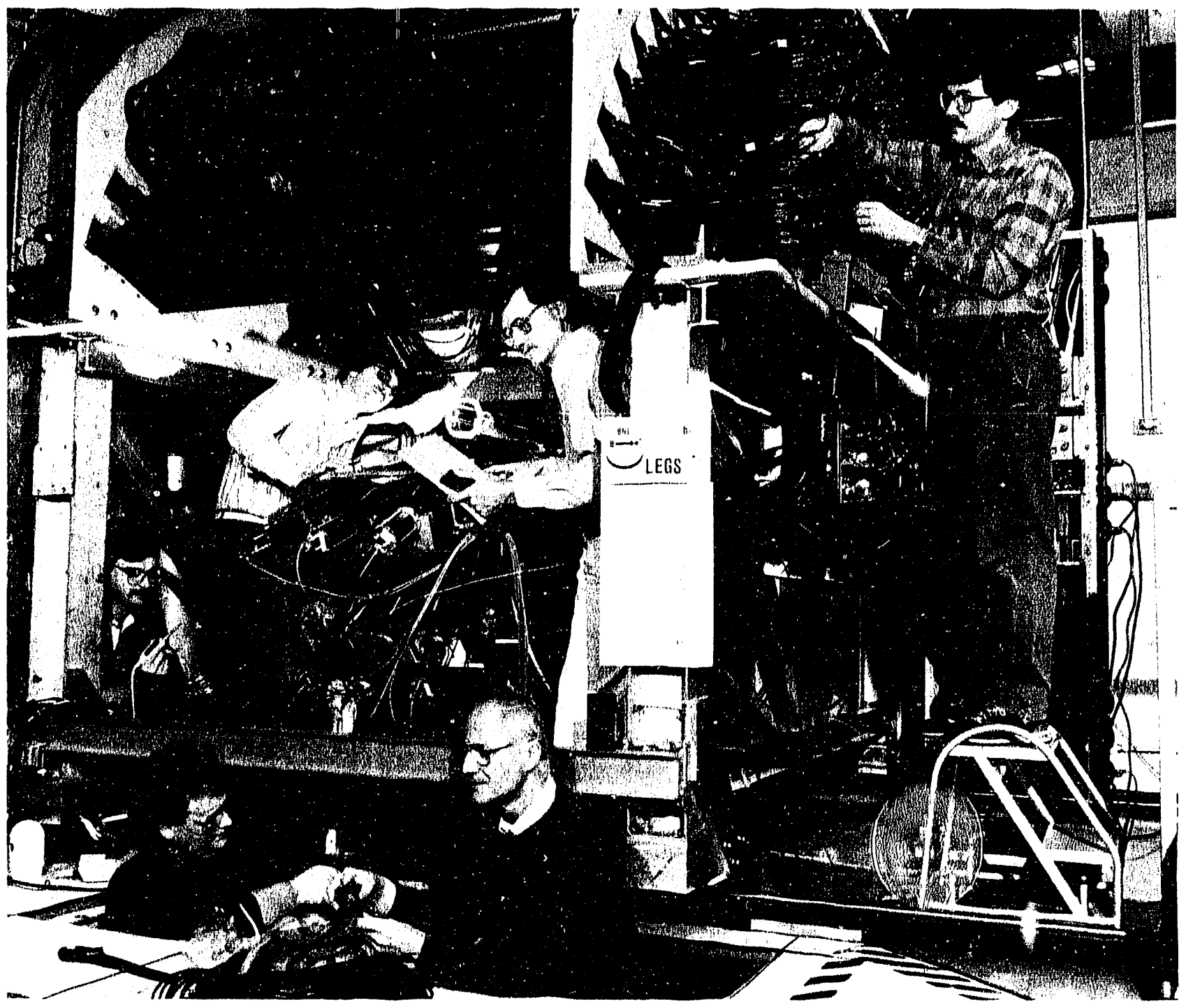




\section{Beamline X8A}

Los Alamos National Laboratory beamline X8A became operational in 1991. This general purpose line was developed to serve a variety of user-provided end stations, particularly for radiometric calibration of experimental systems to be used elsewhere and for scientific experiments in atomic physics, x-ray spectroscopy, and electron spectroscopy. A double focussing mirror and two crystal monochromators provide an end station beam about $2 \mathrm{~mm}$ in size with very low harmonic content and stray light. Full operation to design specs has been achieved. Si(111) crystals provide high resolution from 2 to $6 \mathrm{keV}$ with a flux up to $1 \times 10^{12}$ photons $/ \mathrm{sec}$ at $5 \mathrm{keV}$. Beryl crystals give medium resolution from 0.9 to $2.5 \mathrm{keV}$. W/Si multilayers yield low resolution from 0.26 to $2 \mathrm{keV}$. Several successful calibrations were performed, including one that resolved a long standing uncertainty in a high temperature plasma diagnostic experiment.

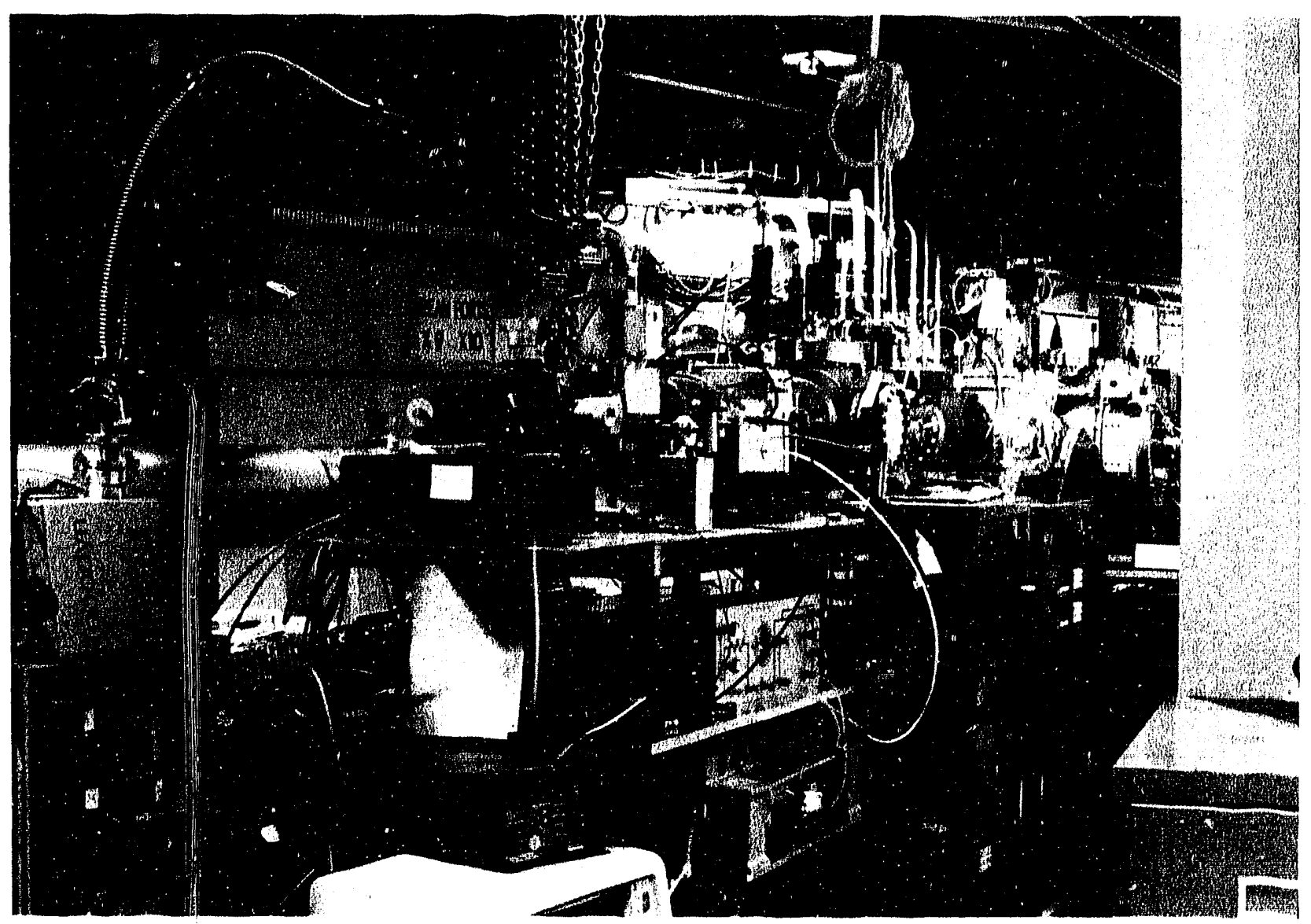

The figure above is a view looking upstream from the end station area on X8A. A user brings his vacuum-compatible end station and attaches to a beamline valve and differential pumping systemi. A user vacuum from $1 \times 10^{-5}$ to $1 \times 10^{-11}$ torr can be accommodated. Shown here is a calibration end station. The experiment to be calibrated is centered in the picture with a three-axis manipulator just to its left; this permits beam profile scanning, imaging calibration of a separate detector, and other functions. Just to the right of the calibration package is the absolute reference detector: here it is gas ion chamber. A differential pumping station upstream of the end station is a vital permanent part of this beamline, which often has end stations with poor vacuum and some detector gases that diffuse through thin windows. A lift table synchronized to the monochromator is also required for most experiments. R. L. Blake, R. G. Hockaday, and M, Sagurton (LANL). 


\section{Beamline X9}

Since 1960, the advances in crystallography studies of proteins have made great progress and have permitted the interpretation of enzyme structures at atomic resolution. This enables a discussion of the reaction mechanisms in terms of positions of atoms and atomic groups. Recently, X-Ray Absorption Spectroscopy (EXAFS) has become a valuable technique in determining the distances of metal centers of proteins in solution state. EXAFS, in combination with any transient technique like stopped flow, flash photolysis, and temperature jump methods, potentially provides information about dynamic structures of proteins during the course of reactions.
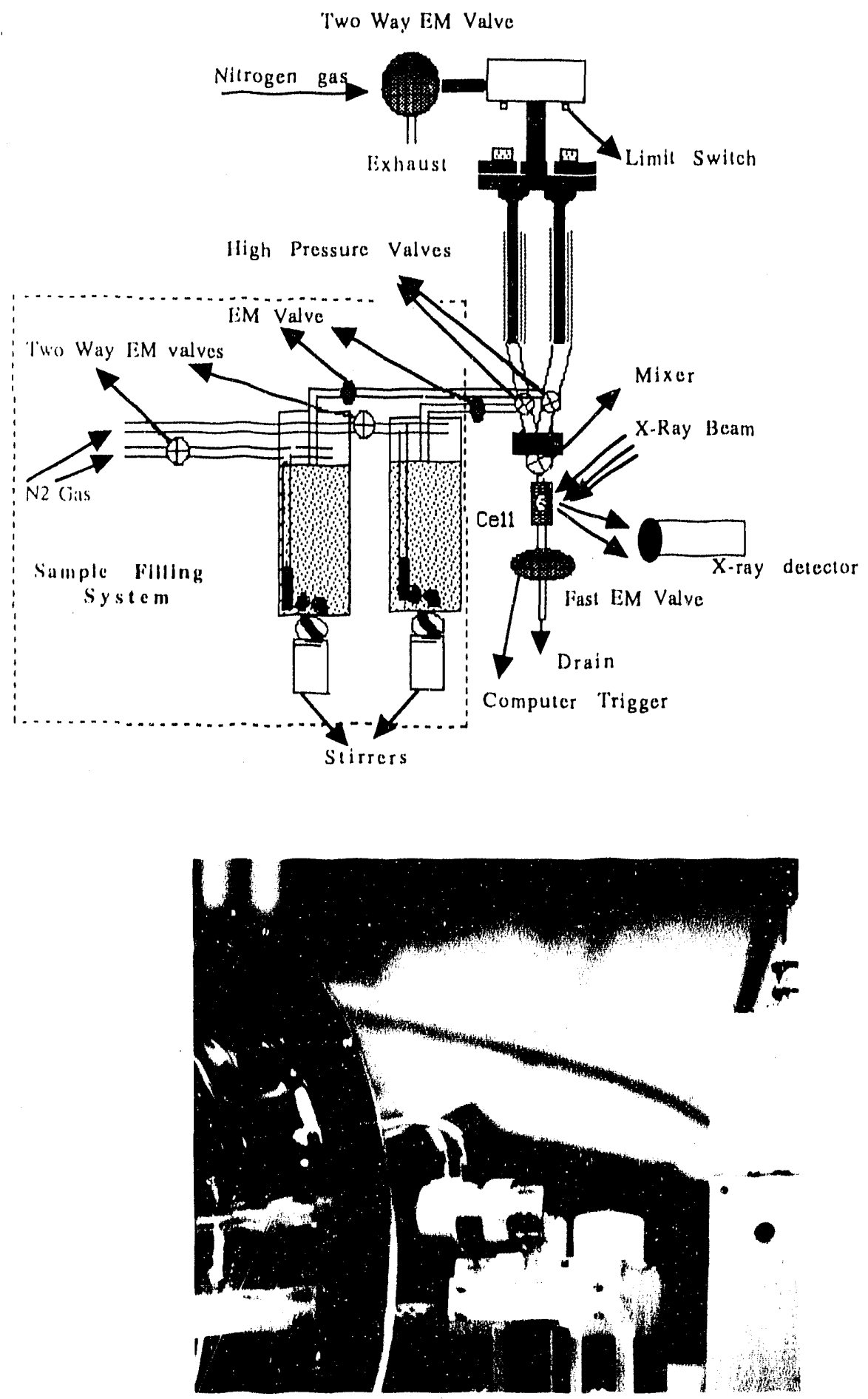

A schematic diagram of the stopped flow unit. It has a mixing time of $5-7 \mathrm{msec}$ and is capable of operating between $-10^{\circ} \mathrm{C}$ to $+40^{\circ} \mathrm{C}$. A number of oxygen sensitive reactions could be studied when the samples were filled into syringes under inert atmospheric conditions.
Stopped flow apparatus during an EXAFS experiment on X9A. A large area photomultiplier tube (PM'T) with $\mathrm{ZnS}$ phosphor was used to collect the $\mathrm{x}$-ray fluorescence signal. Also shown is ait R928 Hammammatsu PMT for reflectance optical monitoring of a sample during EXAFS data collection. 


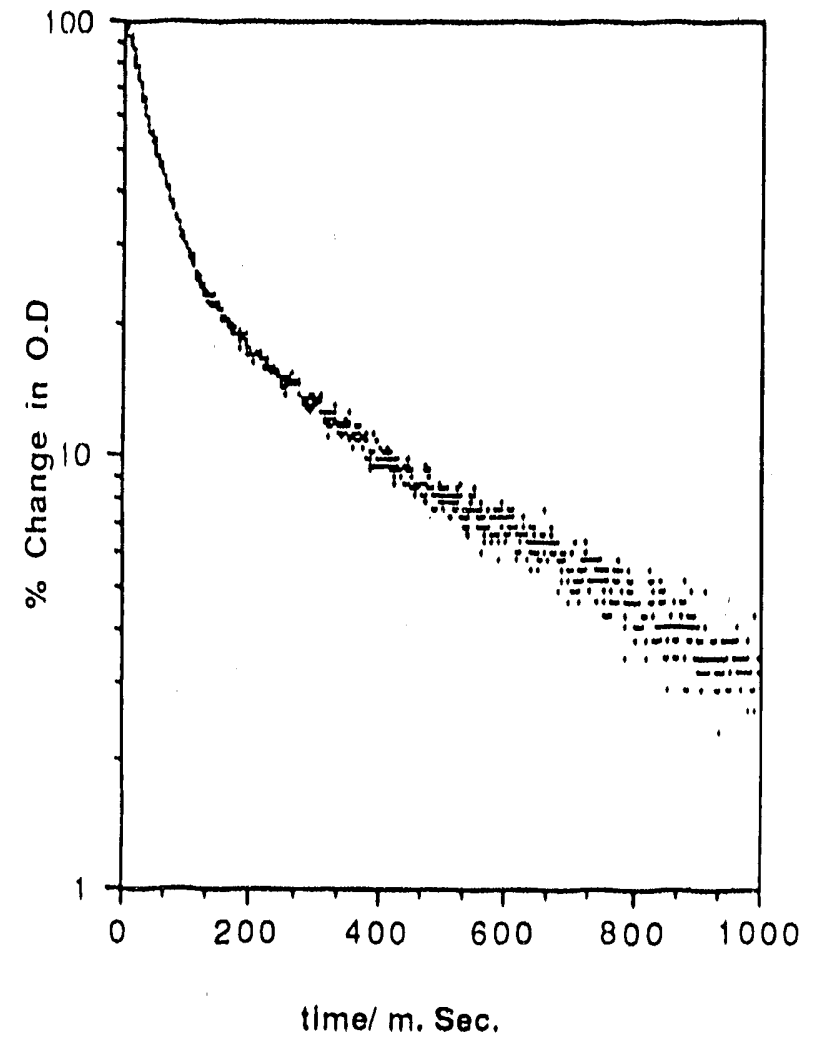

The optical signal at $390 \mathrm{~nm}$ for a pyridinium chlorochromate ion. The reaction produces $\mathrm{Cr}^{3+}$ from $\mathrm{Cr}^{6+}$ ion during the course of $\mathrm{CH}_{3} \mathrm{OH}$ $\mathrm{HCHO}$ oxidation.

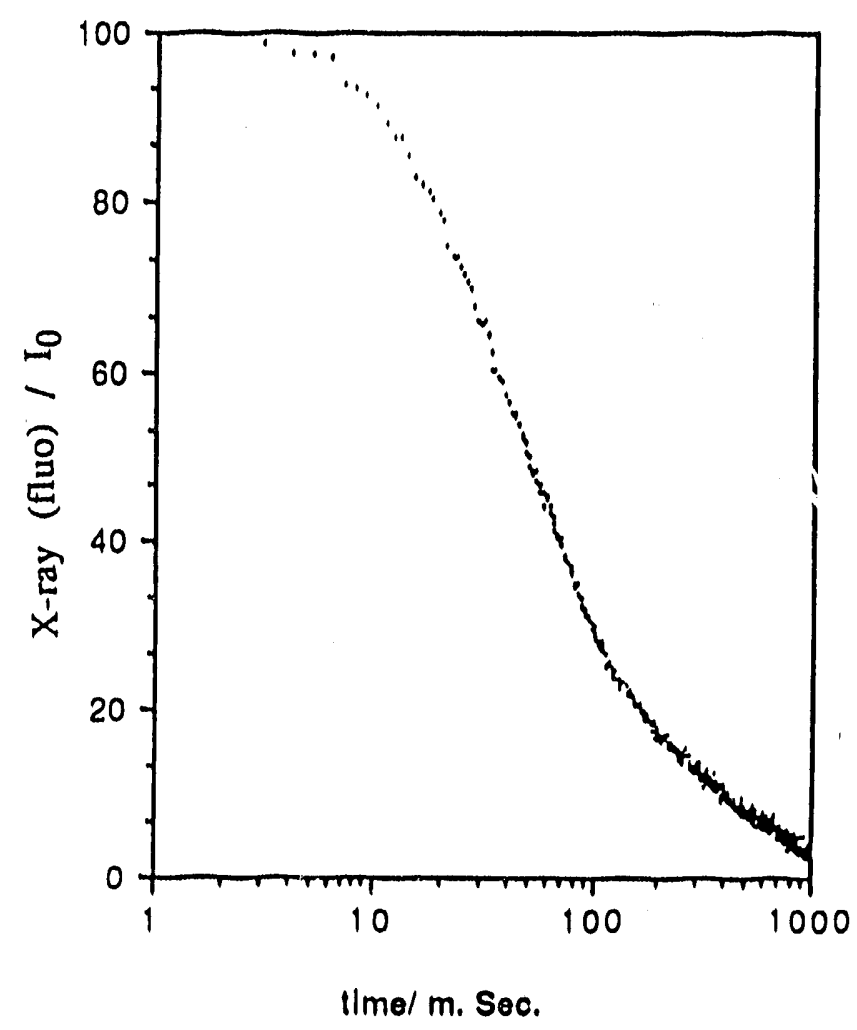

The $\mathrm{x}$-ray fluorescence signal for a $20 \mathrm{mM}$ chromate ion sample monitored at $5.996 \mathrm{keV}$. A substantial change in the near edge structure makes it ideal to calibrate the EXAFS-stopped flow instrument.

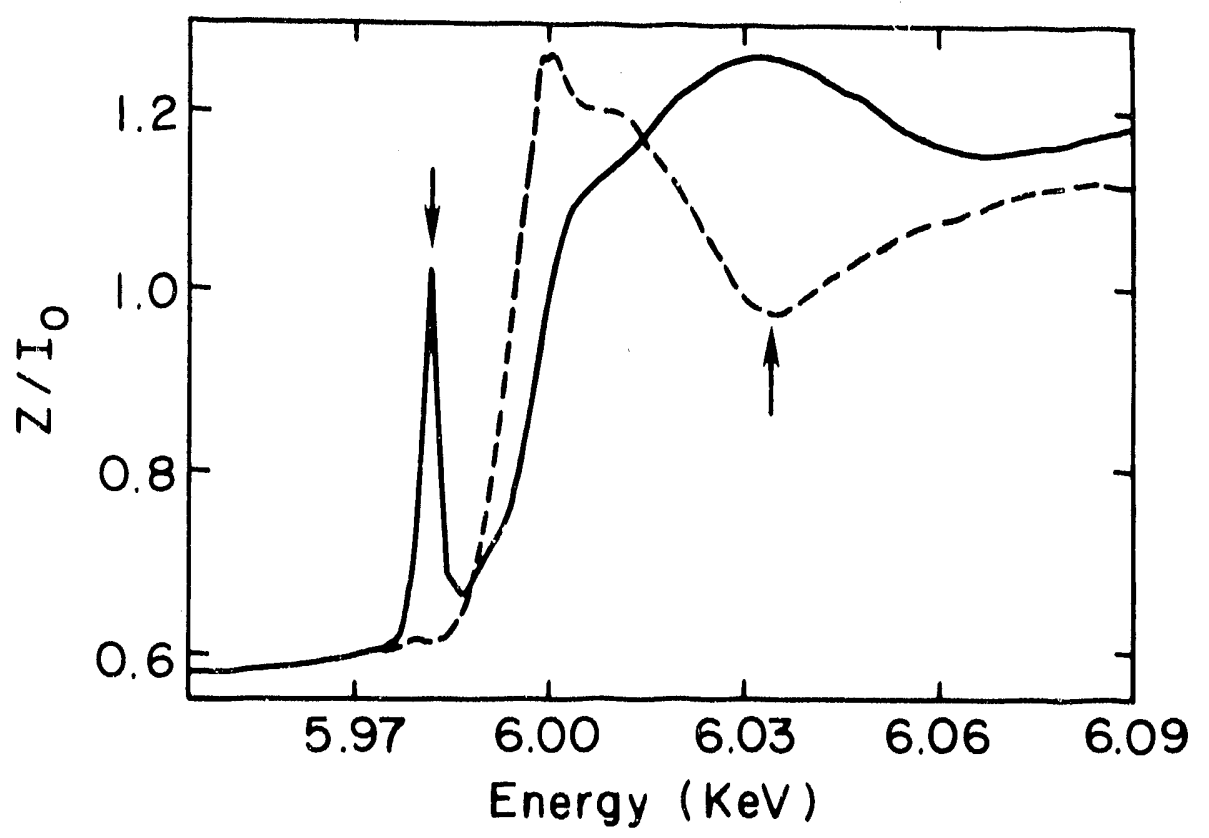

EXAFS spectra collected before and after the reaction from a large number of kinetic profiles shown above. 


\section{Beamline X10A}

Couette Shear Cell designed for in-situ x-ray scattering of macromo ecular fluids under flow. The purpose of this research program is to explore, via in-situ x-ray scattering techniques, new non-equilibrium steady-state structures of cornplex fluids that are produced under shear flow, due to the continuous input and dissipation of energy. Such experiments are among the few quantitative microscopic studies, probing length scales $\leq 10 \AA$ and as large as $\sim \mu \mathrm{m}$ of fluid materials under flow, and will lead to our understanding of phases and phase transitions away from equilibrium, and more generally of non-equilibrium statistical mechanical phenomena. For details see: C. R. Safinya, E. B. Sirota, and R. Plano, "The Nematic to Smectic-A Phase Transition under Shear Flow: A Non-Equilibrium Synchrotron X-ray Study", Phys, Rev. Lett. 66, 1986 (1991).

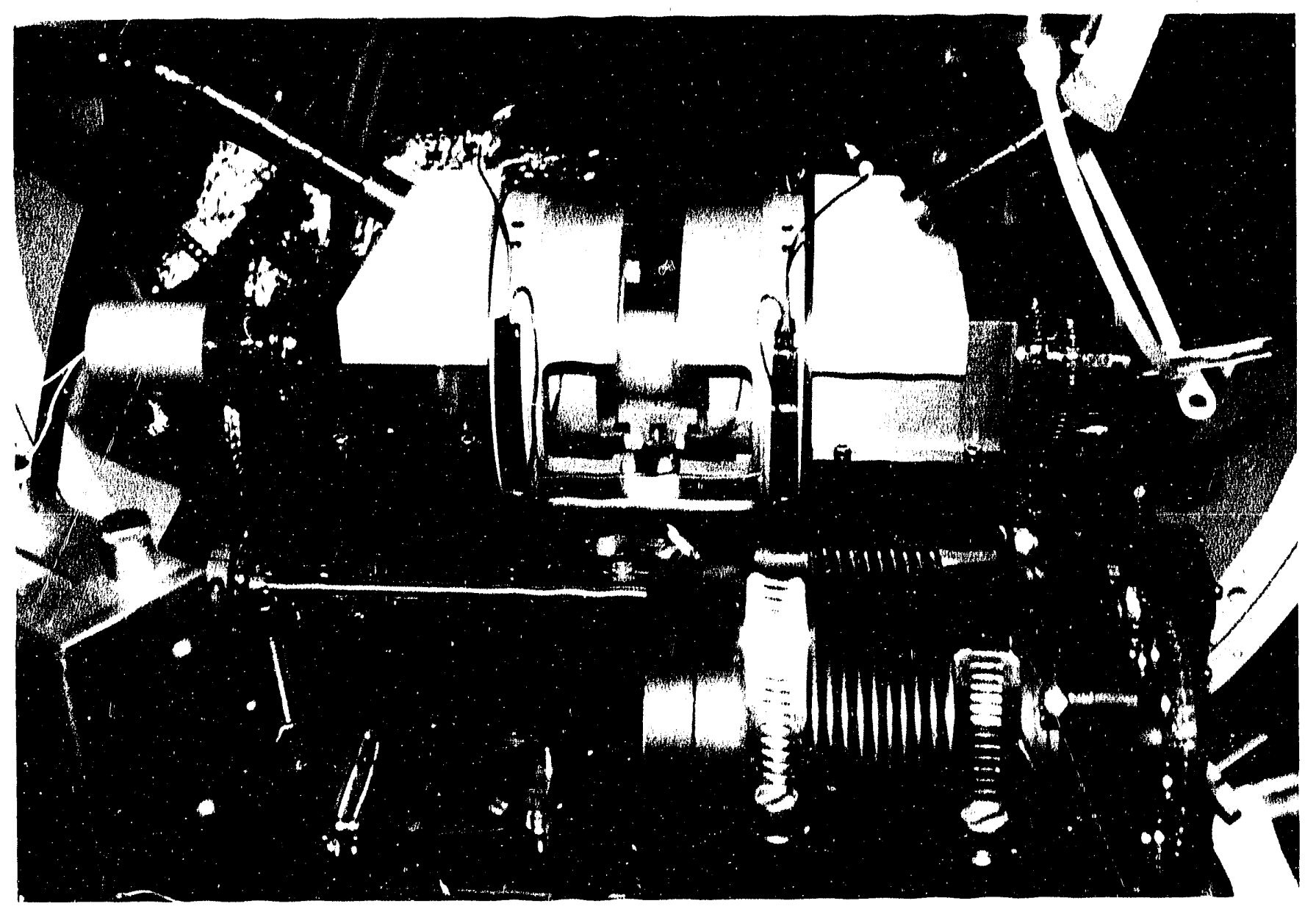




\section{Beamline X10C}

\section{Mirror Feedback System}

Exxon's X10C beamline is designed specifically for x-ray absorption spectroscopy and anomalous x-ray diffraction. The beamline consists of horizontal and vertical entrance slits, UHV double crystal monochromator with AC feedback and a bent-cylindrical focusing mirror.

Many experimental techniques on focused beamlines require increased positional stability. These experiments are not limited by photon flux but by statistical and non-statistical errors in electron beam fluctuations, optic vibrations, monochromator tracking errors and angular deviations resulting from monochromator crystal heating.

We have developed and successfully tested a mirror feedback system on beamline $\mathrm{X} 10 \mathrm{C}$ which is designed to dynamically minimize vertical beam motion at the sample position and compensate for these errors. The systern functions by monitoring the beam position near the sample location with a position sensitive ion chamber coupled through a feed. back loop to a piezoelectric trunsducer controlling the mirror tilt angle. The technique will reduce $\pm 0.5 \mathrm{~mm}$ beam deviation to \pm 0.5 microns from $D C$ to $10 \mathrm{~Hz}$. This system has enhanced the quality of fluorescence and transmisson XAFS data for very small samples, extremely dilute samples and inhomogeneous samples.
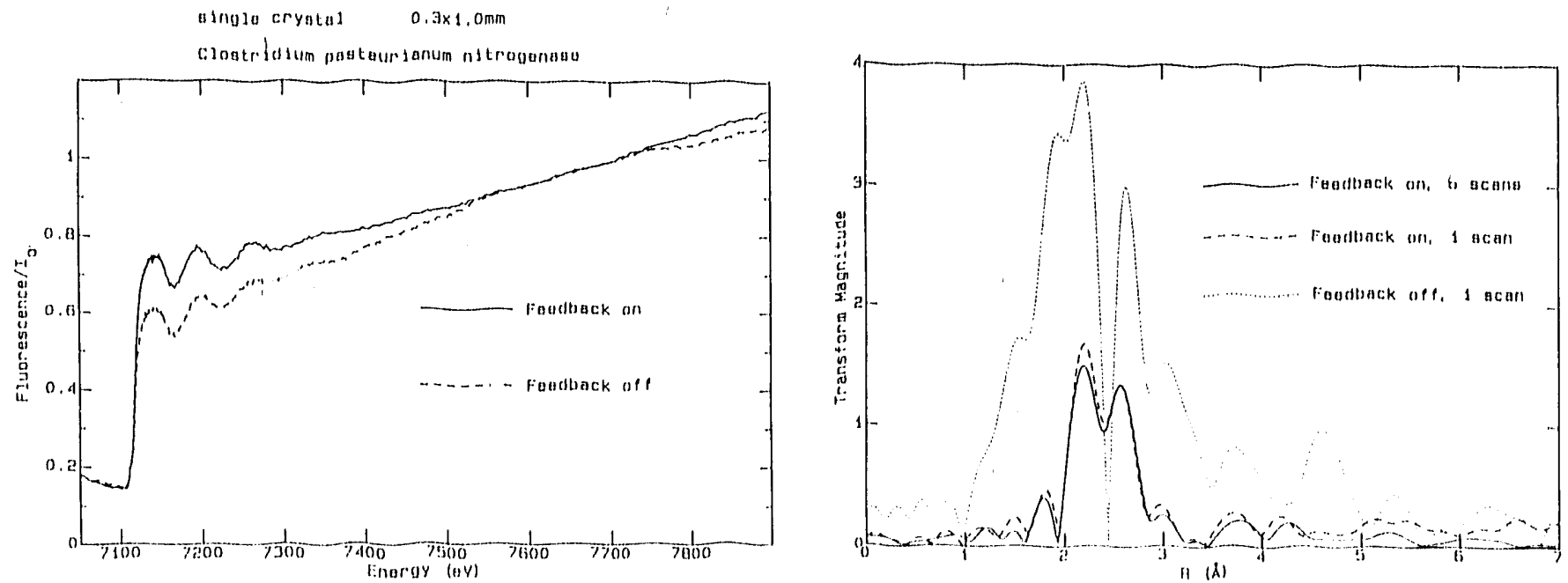

The figures above demonstrate the improvement in quality of XAFS data by using a mirror feedback system for a 0.3 $\mathrm{mm}$ by $1.0 \mathrm{~mm}$ single crystal sample. In this case, the vertical focus is of the same order as the sample size and small beam motions during the energy scan severely distort the Fourier transform. The experiment was performed in the fluorescence mode with a 13 element Ge-detector.

We are also developing a new electronics for an AC feedback system which locks on the peak of the Darwin rocking curve and dynamically compensates for monochromator crystal heuting. The feedback electronics uses an amplifier stage with preset dead bands, and an automatic gain control (AGC) stage to modify the loop-gain through a transfer unction which significantly extends dynamic range, reduces noise and enhances sensitivity. Mike Sansone (Exxon). 


\section{Beamline X11A}

\section{Focusing Crystal}

Photograph of slotted triangular crystal employed for sagittal focusing at beamline X11A at the NSLS. The crystal was cut from a $0.76 \mathrm{~mm}$ Si wafer into a triangle of base-width $66.5 \mathrm{~mm}$ and height $85 \mathrm{~mm}$. Grooves were machined into the crystal face using a $0.05 \mathrm{~mm}$-wide diamond saw, such that the thickness of the weak links was $0.1 \mathrm{~mm}$. The face of the crystal has 74 grooves and thus 73 reflecting surfaces of width $0.75 \mathrm{~mm}$. The crystal is surrounded by an aluminum bracket to protect the crystal during handling. S. M. Heald (BNL Dept. of Appl. Scl.) and G. M. Lamble (NSLS/North Carolina State U.).

a)

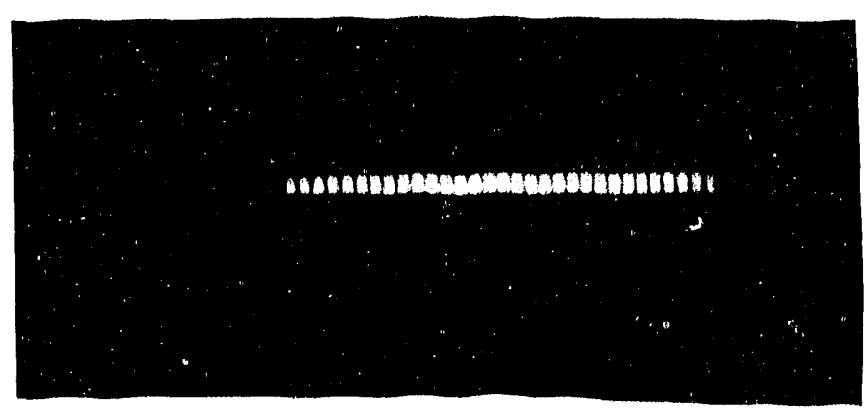

b)

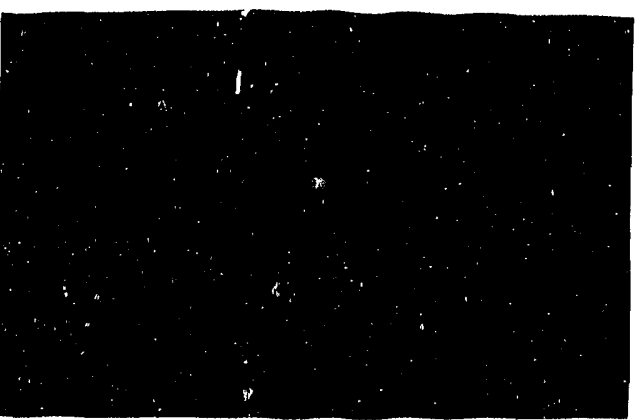

c)

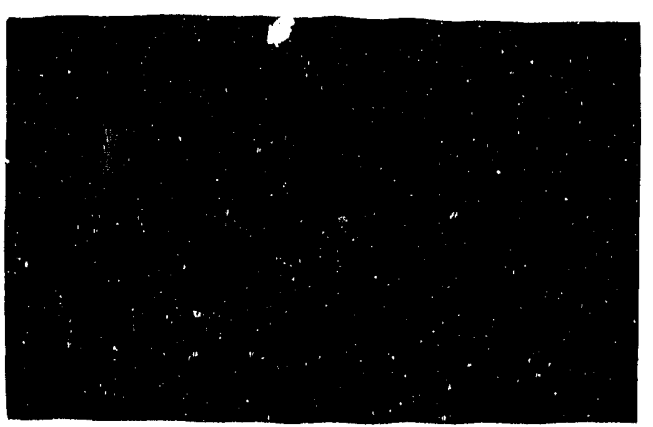

\section{Focusing Crystal Images}

Photograph of the images observed in the X11A hutch: (a) from the focusing crystal when flat, (b) the focused beam with a pre-monochromator slit height of $1 \mathrm{~mm}$, and (c) the focused beam with a pre-monochromator slit height of $0.5 \mathrm{~mm}$. The focused beam in case (c) is about $1.5 \mathrm{~mm}^{2}$. The intensities achieved by focusing over normal flat-crystal running, with a $1 \mathrm{~cm}$ hutch slit-width, vary between a factor of four and two over the present operating energy range of 6 to $11 \mathrm{keV}$. S. M. Heald (BNL Dept. of Appl. Sci.) and G. M. Lamble (NSLS/North Carolina State U.). 


\section{Beamline X17B2}

A 44 year old male sustained an acute myocardial infarction on Feb, 19, 1991, On Feb. 25, cardiac catherization revealed an $80 \%$ stenosis of the Right Coronary Artery (RCA) - see left hand CINE imago. Balloon angioplasty was per* formed to re-open the RCA - see middle CINE inuge. Transvenous coronary angiography was performed on Aug. 22, 1991 at the Synchrotron Medical Research Facility (SMERF) showing that the RCA was still patent - see right hand SMERF image, Left Anterior Oblique $40^{\circ}$ view. Beam parameters: X17 superconducting wiggler field was 3.0 Tegla; ring current was $206 \mathrm{~mA}$.

\section{CONVENTIONAL SOURCE}

\section{ARTERIAL CATHETER}

\section{SYNOHROTRON IMAGE}

\section{VENOUS CATHETER}

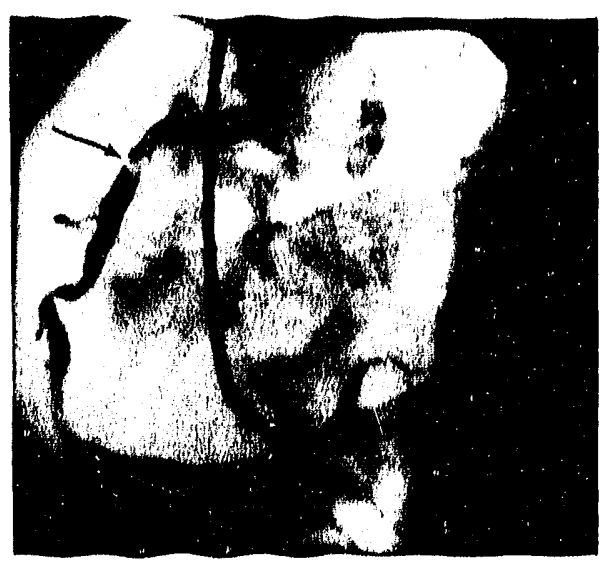

PRE-

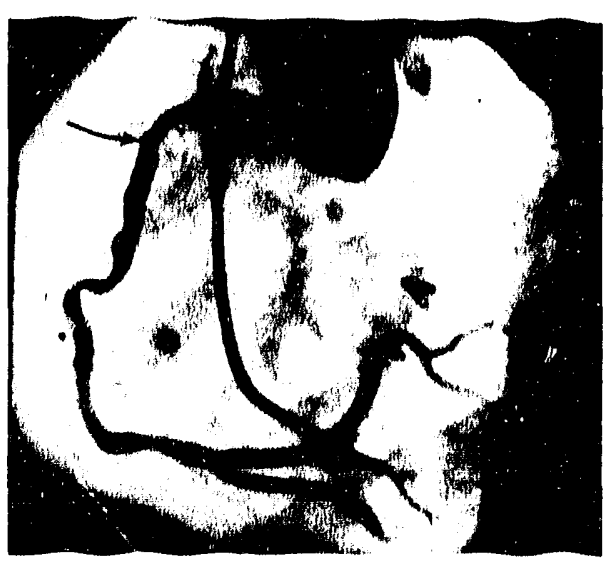

POST-

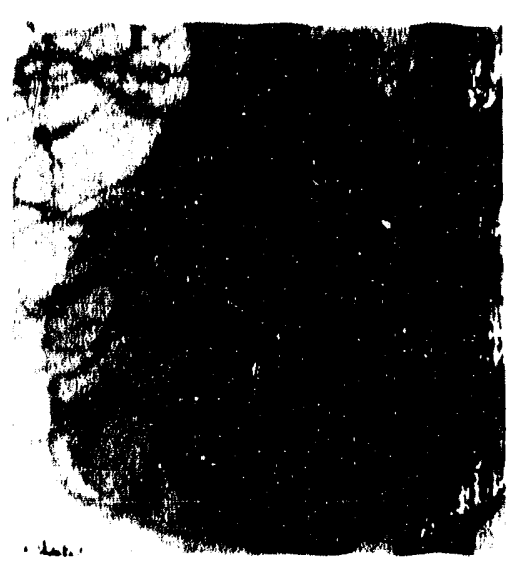

6 MONTHS 


\section{Beamline X17C}
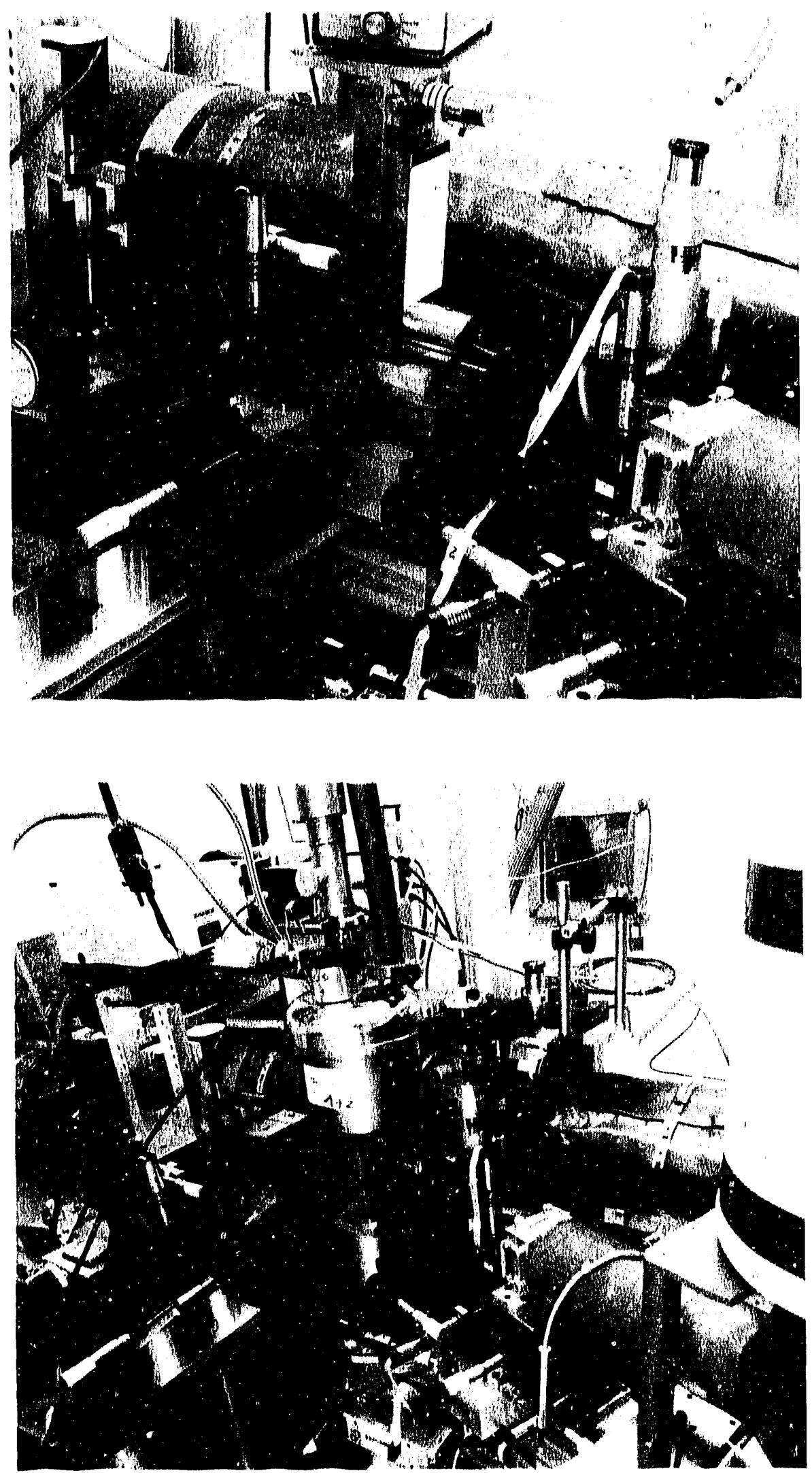

The polychromatic beamline X-17C is equipped with an energy dispersive x ray diffruction (EDXD) sys. tem for probing ultra-small samples in high-pressure diamond anvil cells. The incident beam over an en* ergy range of 5 to $100 \mathrm{keV}$ is controlled by a double slit sytem made of tungsten and tantalum that is capable of producing uniform beam sizes of 5 to $10 \mu \mathrm{m}$ impinging on samples in a diamond anvil cell. The alignment of the incident beam, sample, and diffracted beam are achleved by an automated $\Theta \cdot 2 \Theta$ sys* tem with a positional accuracy of $1 \mu \mathrm{m}$ and angular accuracy of $0.001^{\circ}$. $\mathrm{X}$-ray diffraction of polycrystalline samples at multimegabar pressure range and at temperatures of $50-3000 \mathrm{~K}$ have been performed. J. Z, Hu et al. (Carnegie Inst. of Washington).

A cryogenic system for singlecrystal EDXD at high pressures. The sample, which is a single-crystal helium, is grown from its fluid phase at $11 \mathrm{GPa}$ at room temporature in a "membrane" diamond cell. The pressure is generated by a mem. brane press using gaseous helium as the hydraulic iluid. The $\chi$-rotational circle, which contains the diamond cell at its center, is cooled in a liquid. helium cryostat, which in turn is located on the $\theta-2 \theta$ sample stage. The pressure at low temperatures can be measured simultaneously with a ruby fluorescence system which in. cludes a $\mathrm{He}-\mathrm{Cd}$ laser as the excitation radiation source, a microscope for steering the laser to a ruby chip at high pressures and for collecting the fluorescence, and a polychromatic spectrometer for measurement of the pressure-ehift of the ruby $R$-line. P.V-T equation of state, crystallographic structure, and phase diagram of helium have been determined in this system. $R$. Le'Toullec et al.(U. of Paris) and J. Z Hu et al. (Carnegie Inst, of Wasilington). 


\section{Beamline X18A}

The figure shows a UHV chamber at the MATRIX beamline X18A which has recently been completed for structural analysis of adsorbed films and single-crystal surfaces. It is particularly well suited for investigations of physisorbed and other weakly bound films due to its combined in-situ LEED and low-temperature capabilities. The chamber is transportable and mounts on a standard Huber x-ray diffractometer. The utility of these features for studies of the structure and phase transitions of physisorbed films has been demonstrated in some preliminary investigations of the Xe/Ag(111) system. J. R. Dennison (Utah State U.), S.-K, Wang, P. Dai, H. Taub, T. Angot (U. Missouri-Columbia), and S. N. Ehrlich (Purdue U.).

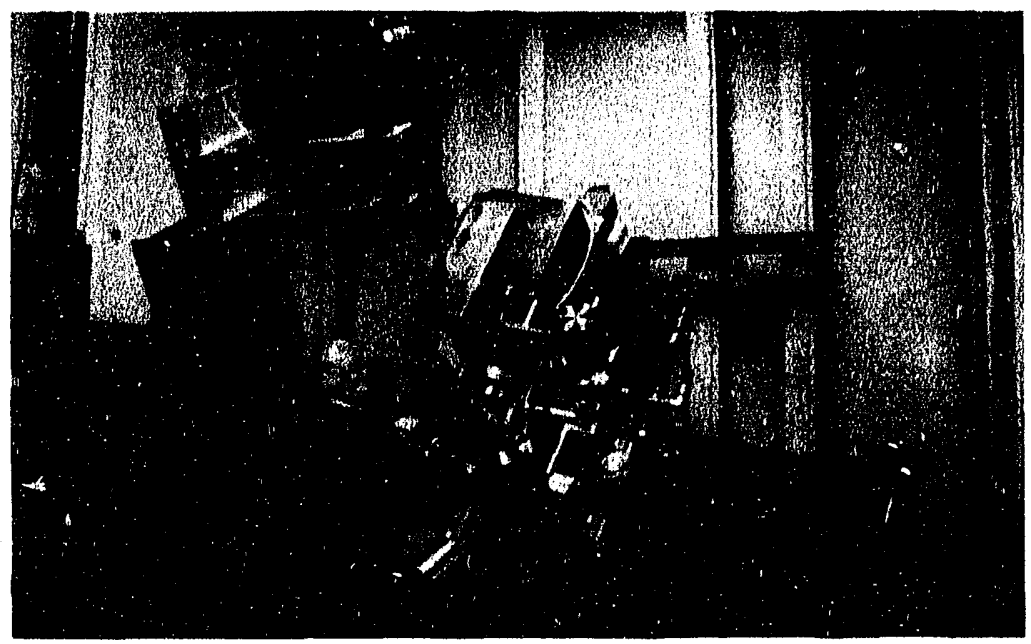

\section{Beamline X23A3}

A new small-angle x-ray scattering instrument, optimized for high throughout at the NSLS, high angular and wavelength resolution, large sample cross-sectional area, accurate energy tuning, excellent signal-to-noise ratio and harmonic rejection has been commissioned on X23A3. The instrument has been used for anomalous scattering measurements near the $\mathrm{Cr} \mathrm{K}$-edge, for measurements of pore-size distributions in ceramics, and for the study of disordered structures, as well as for a number of other materials systems.

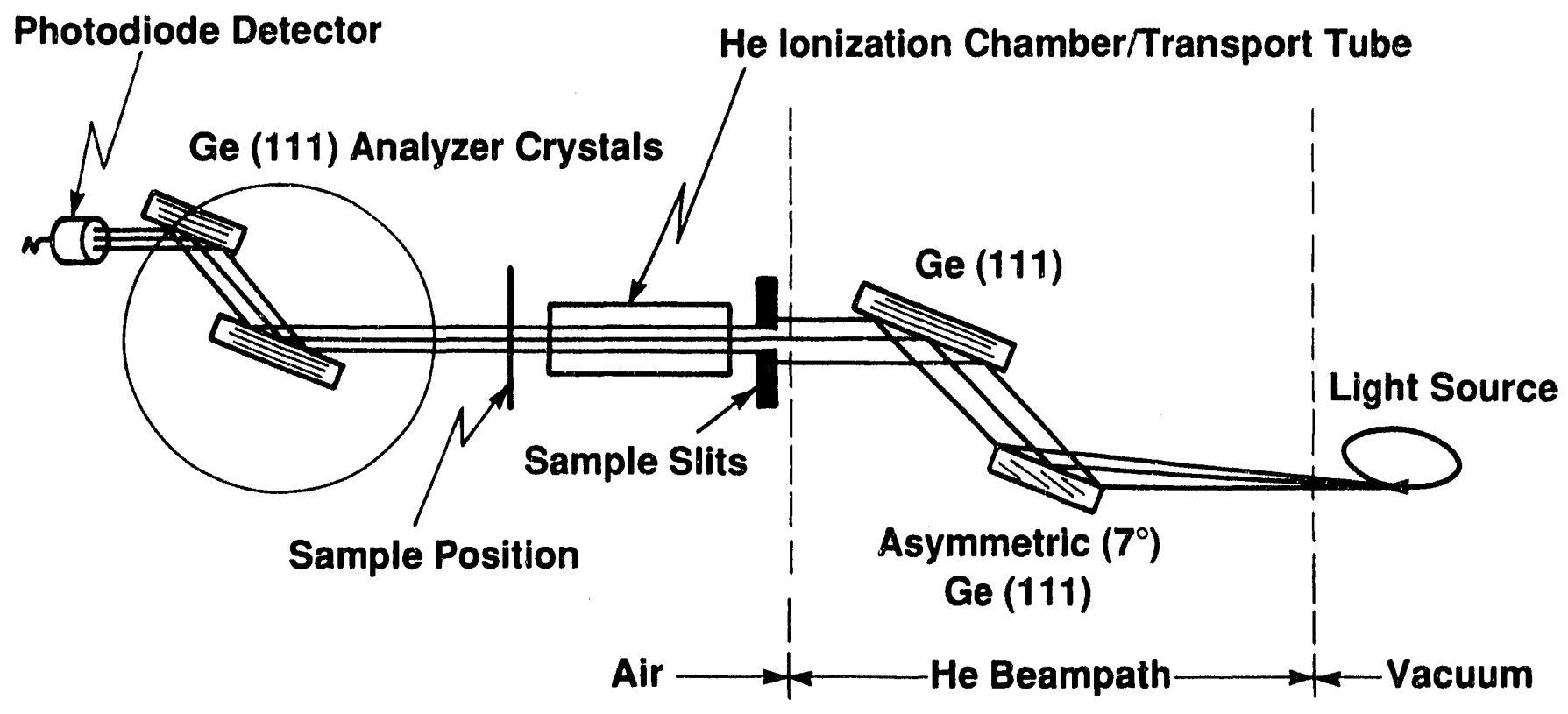




\section{Beamline X25}

\section{Cryogenic X-Ray Optics}

The X25 wiggler white beam, when doubly-focussed, has a power density exceeding $150 \mathrm{~W} / \mathrm{mm}^{2}$ at the focal point. This is a representative density which optics at next-generation synchrotron source undulator beam lines will have to withstand. Crystal monochromators cannot diffract x-rays efficiently, nor preserve the high brightness of the source, unless surface strains are kept within a few arcseconds. This is impossible to achieve for all materials at room temperature in the presence of the X25 doubly-focussed beam. At cryogenic temperatures, however, thermal strains can be re duced substantially, since the thermal expansion coefficient becomes very small and the thermal conductivity increases in many materials. In a joint ESRF/NSLS experiment at X25, the diffraction efficiency of a silicon crystal was measured as a function of temperature, using the doubly-focussed incident beam. Plotted above is the slope error across the crystal surface, determined from $x$-ray rocking curves, compared with the ratio of the thermal expansion coefficient to thermal conductivity. As expected, the observed slope error is close to zero at $100 \mathrm{~K}$, resulting in perfect diffraction efficiency.

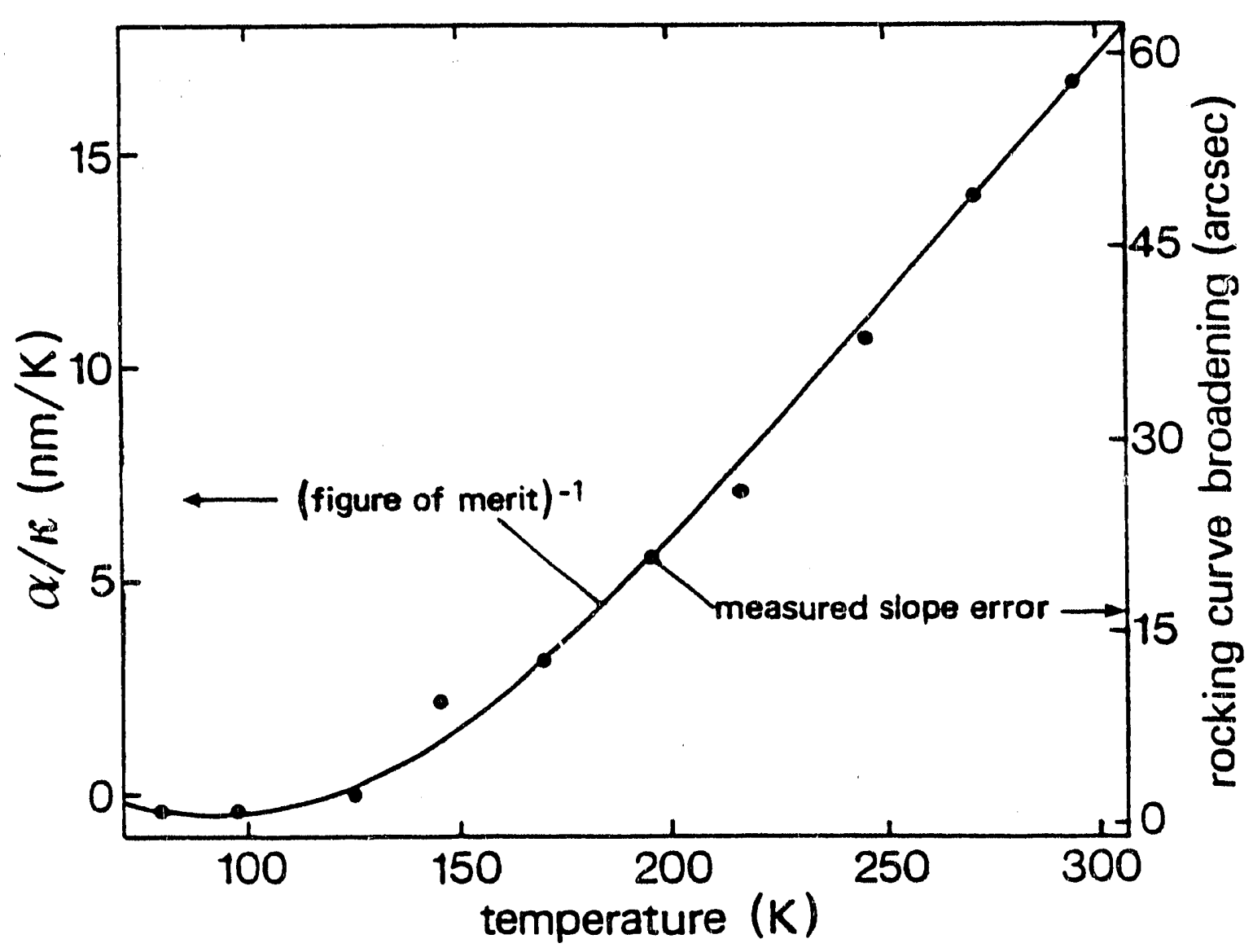



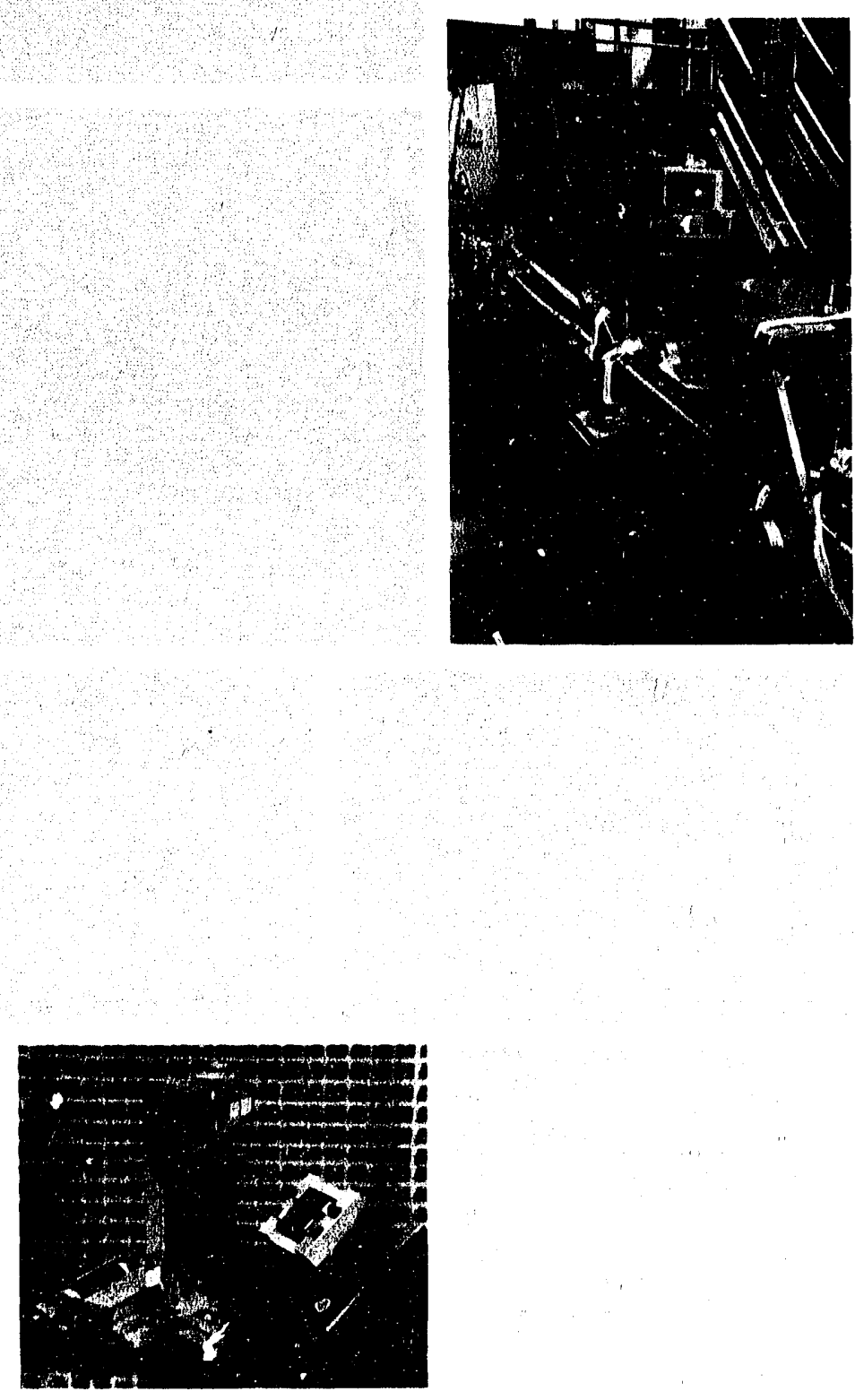


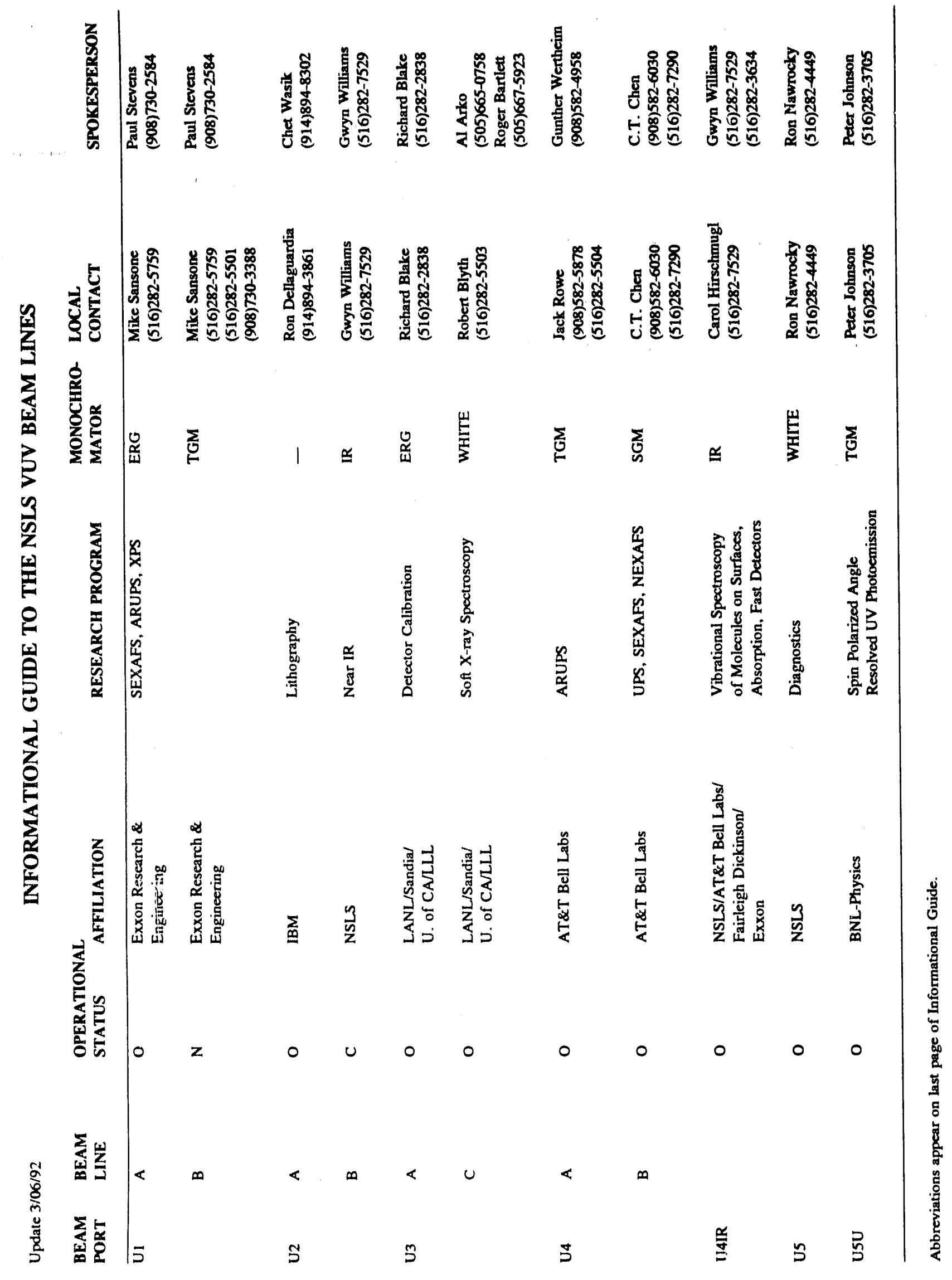




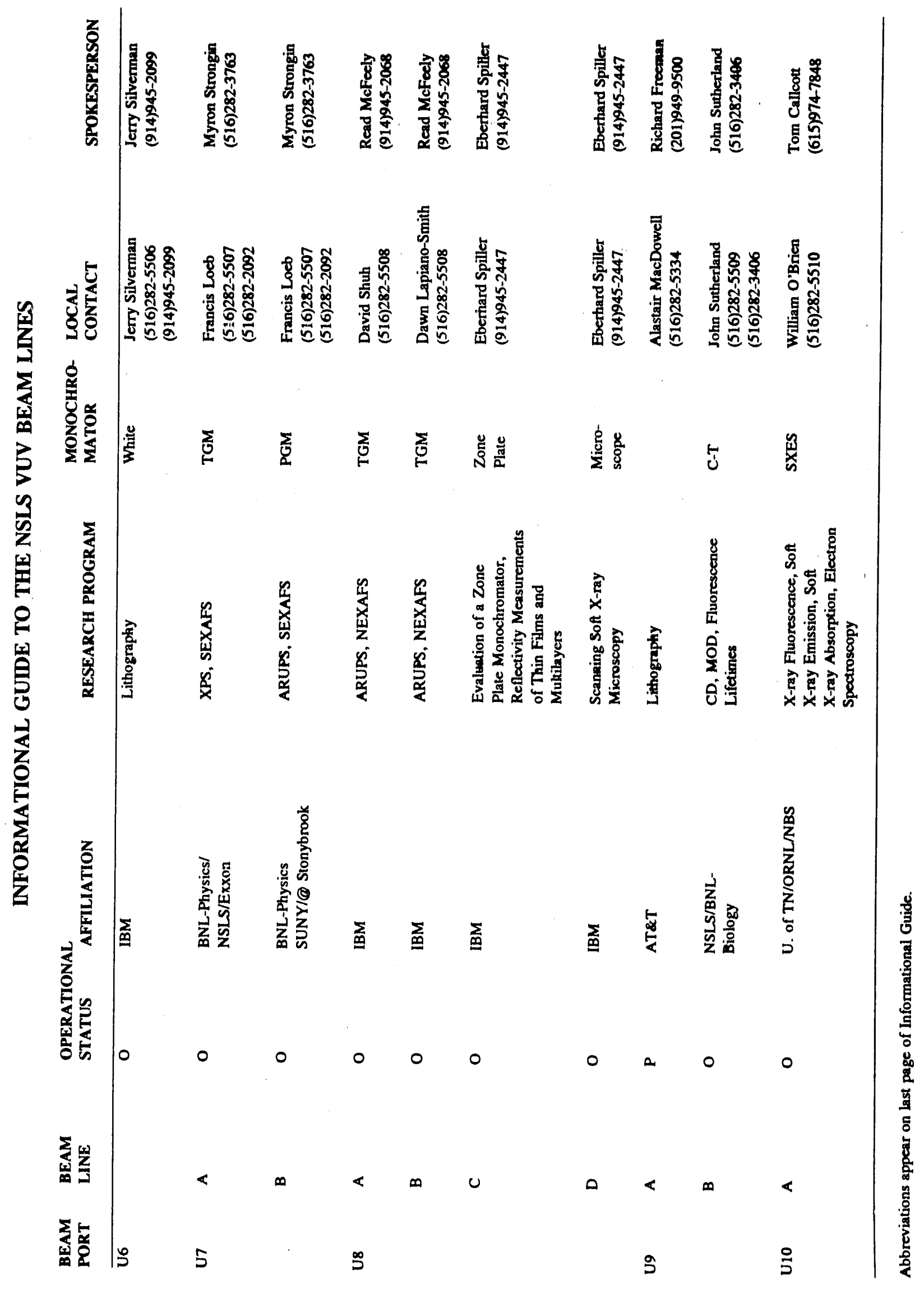




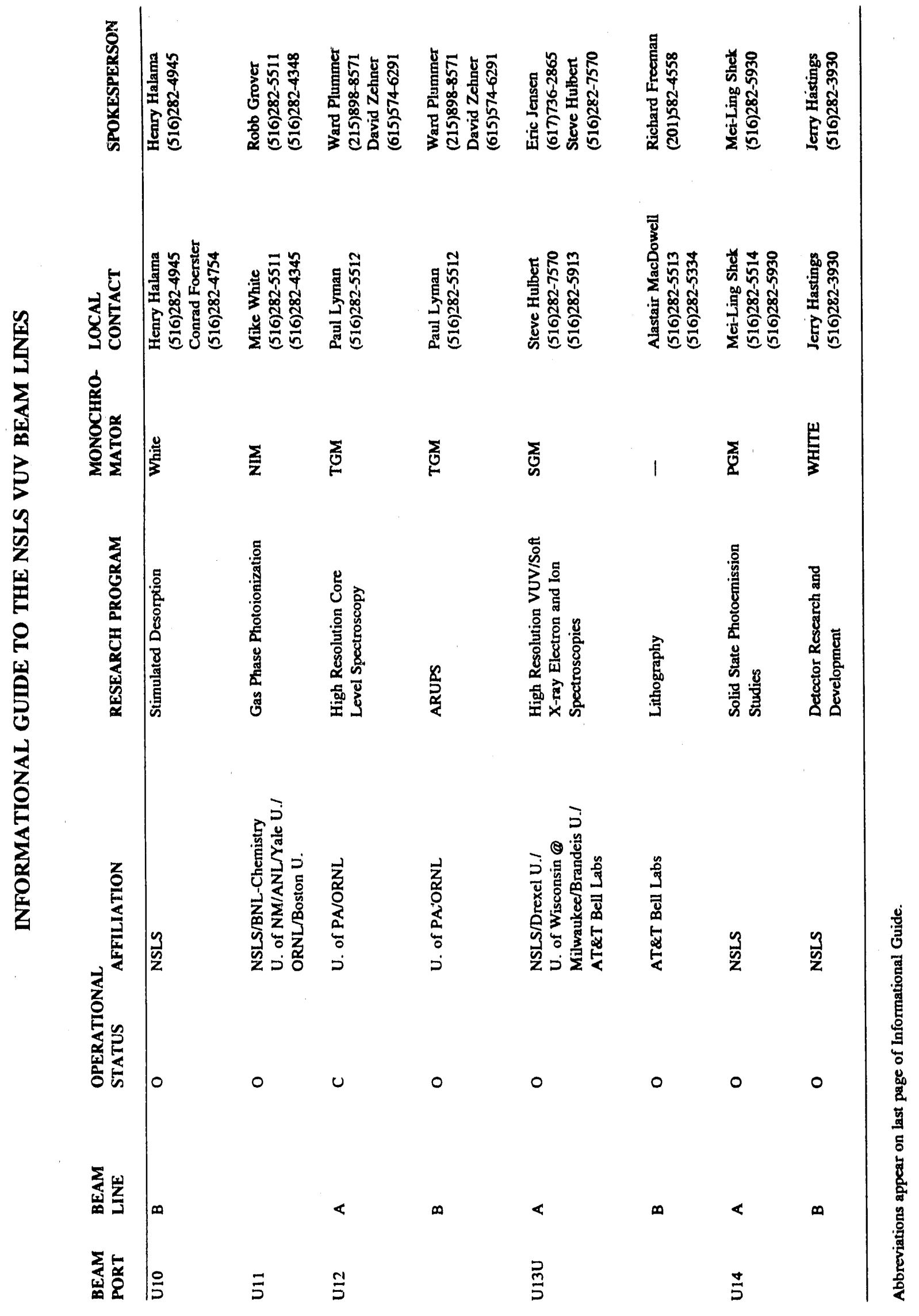




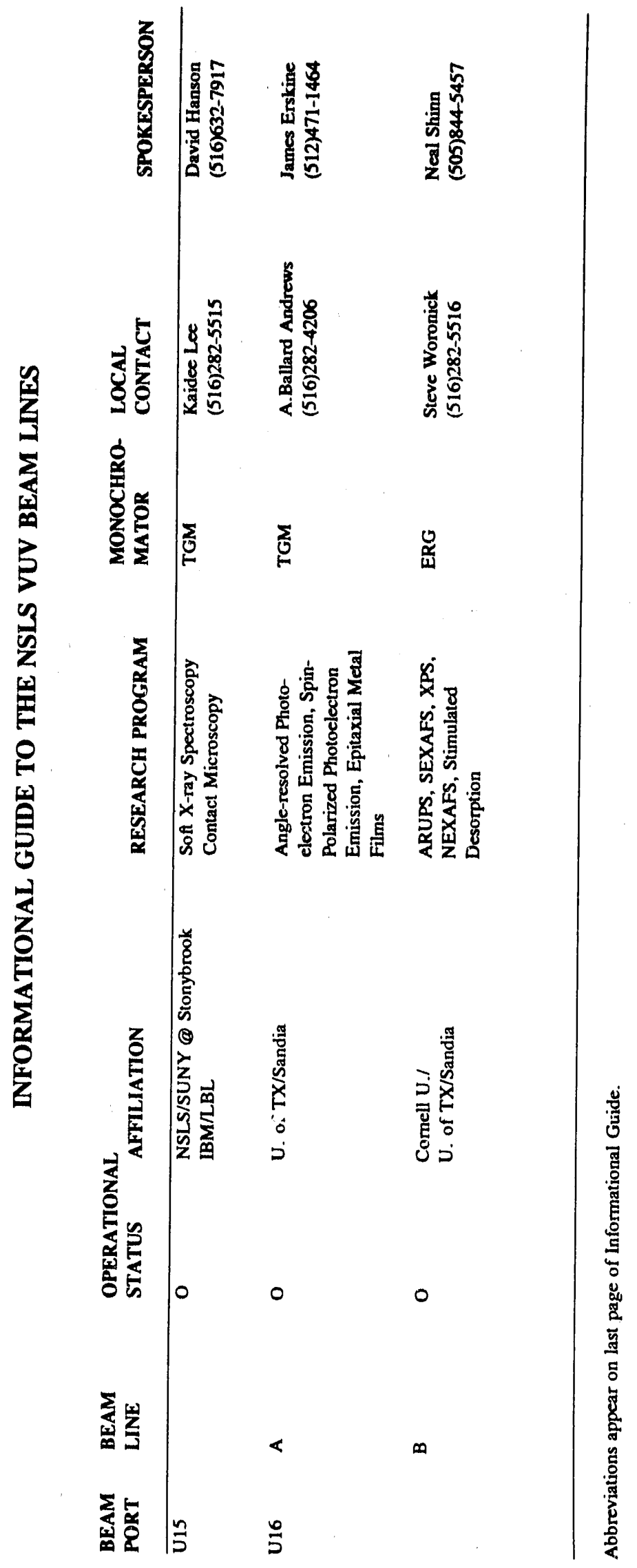




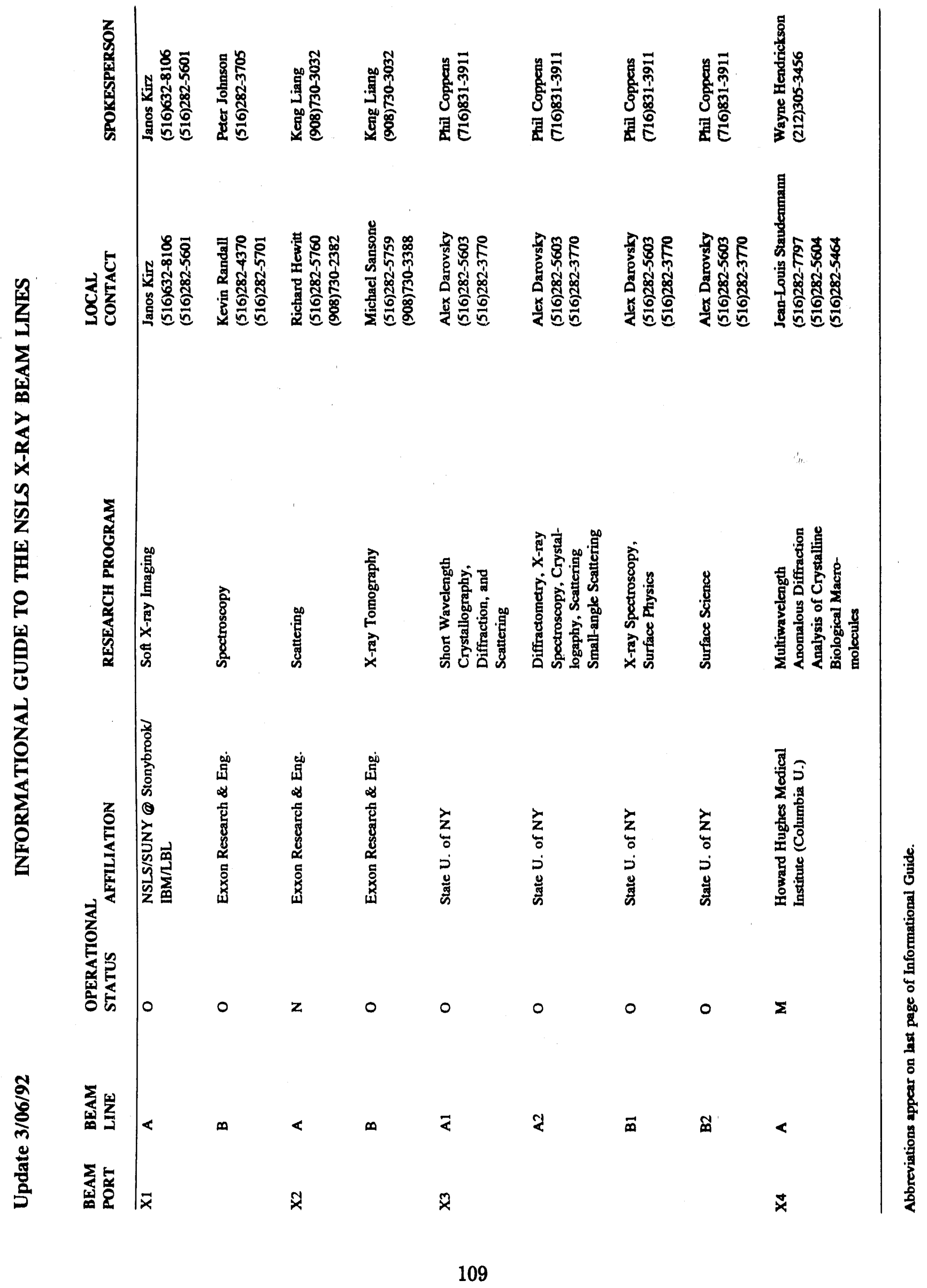




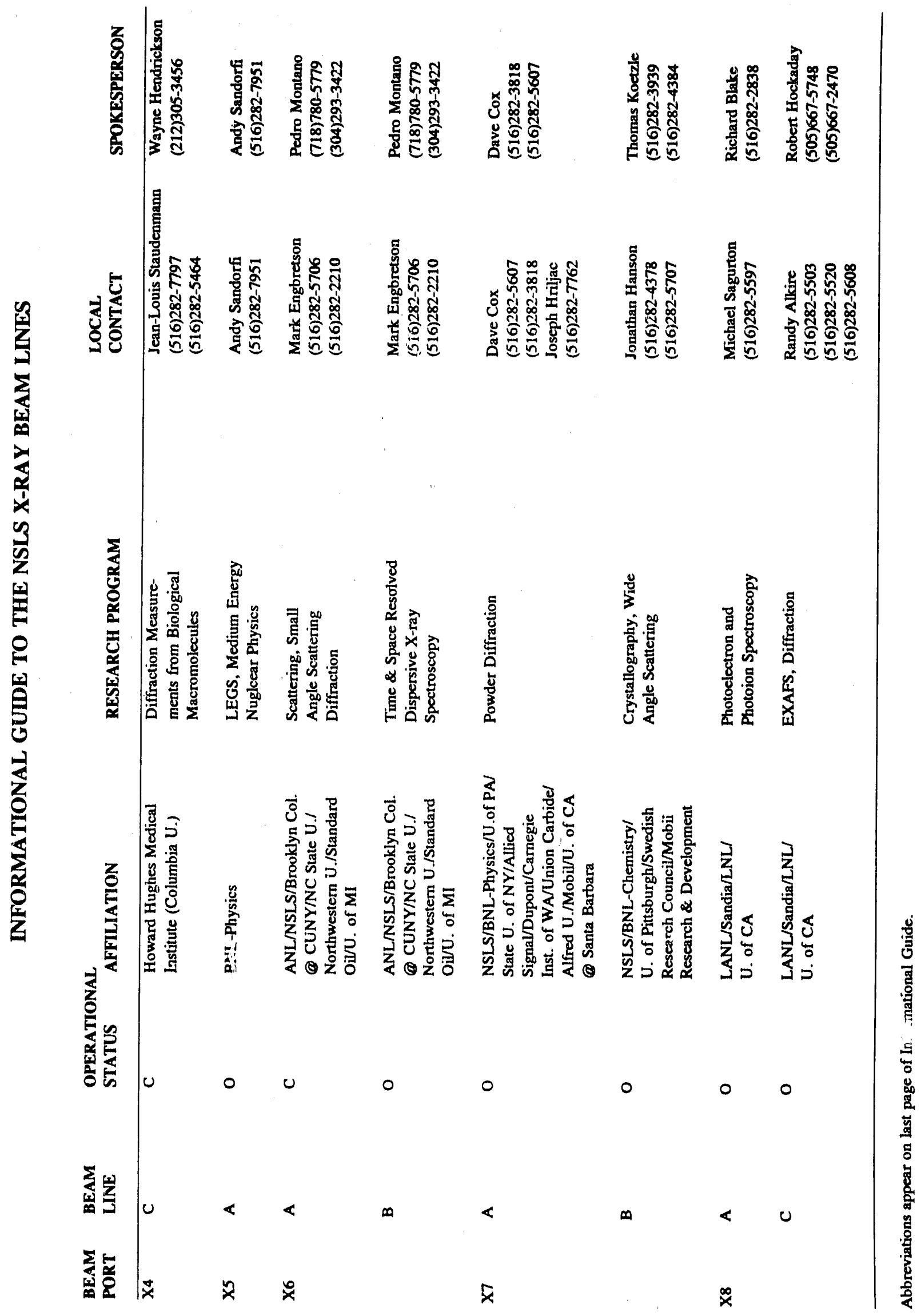




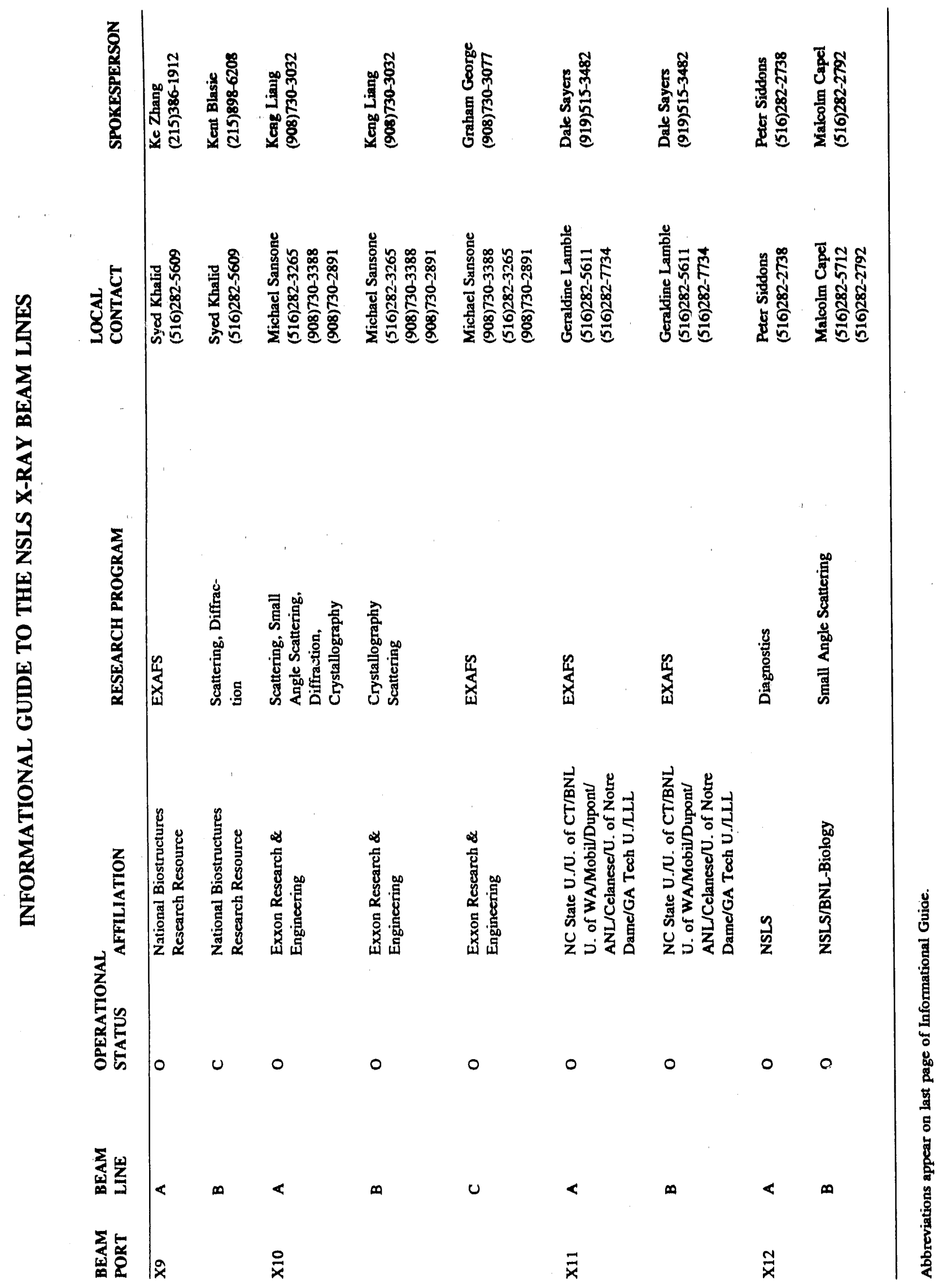




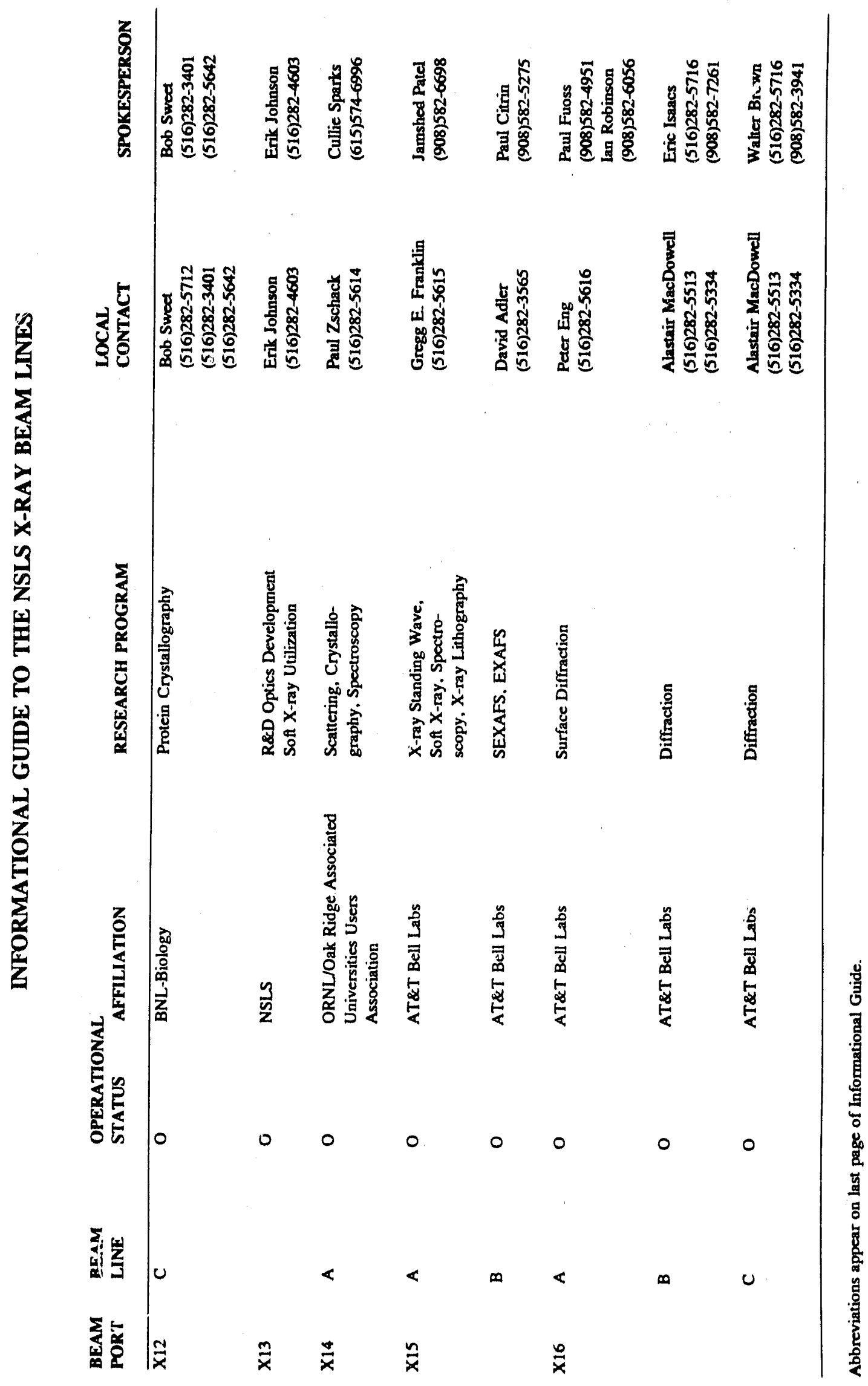




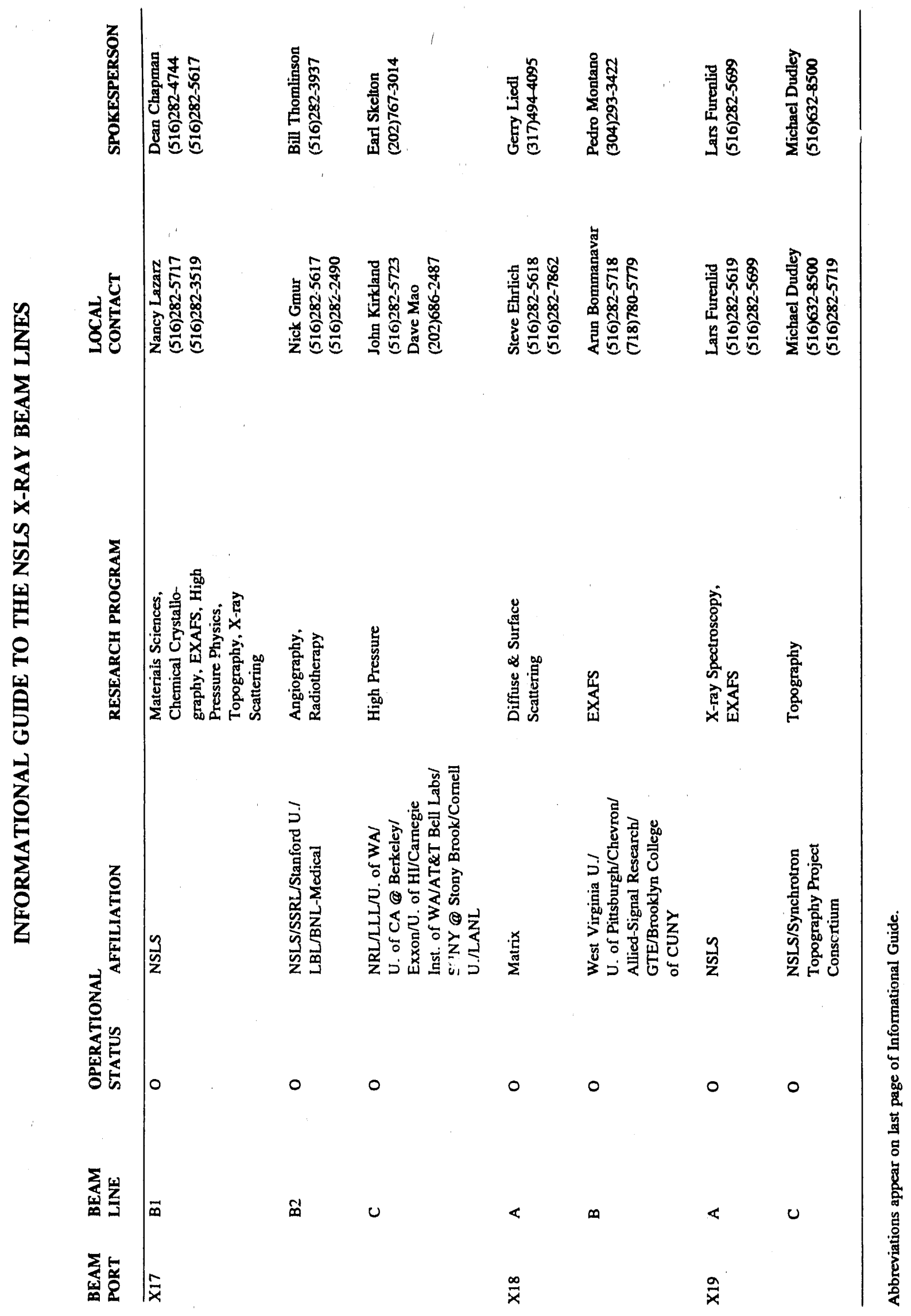




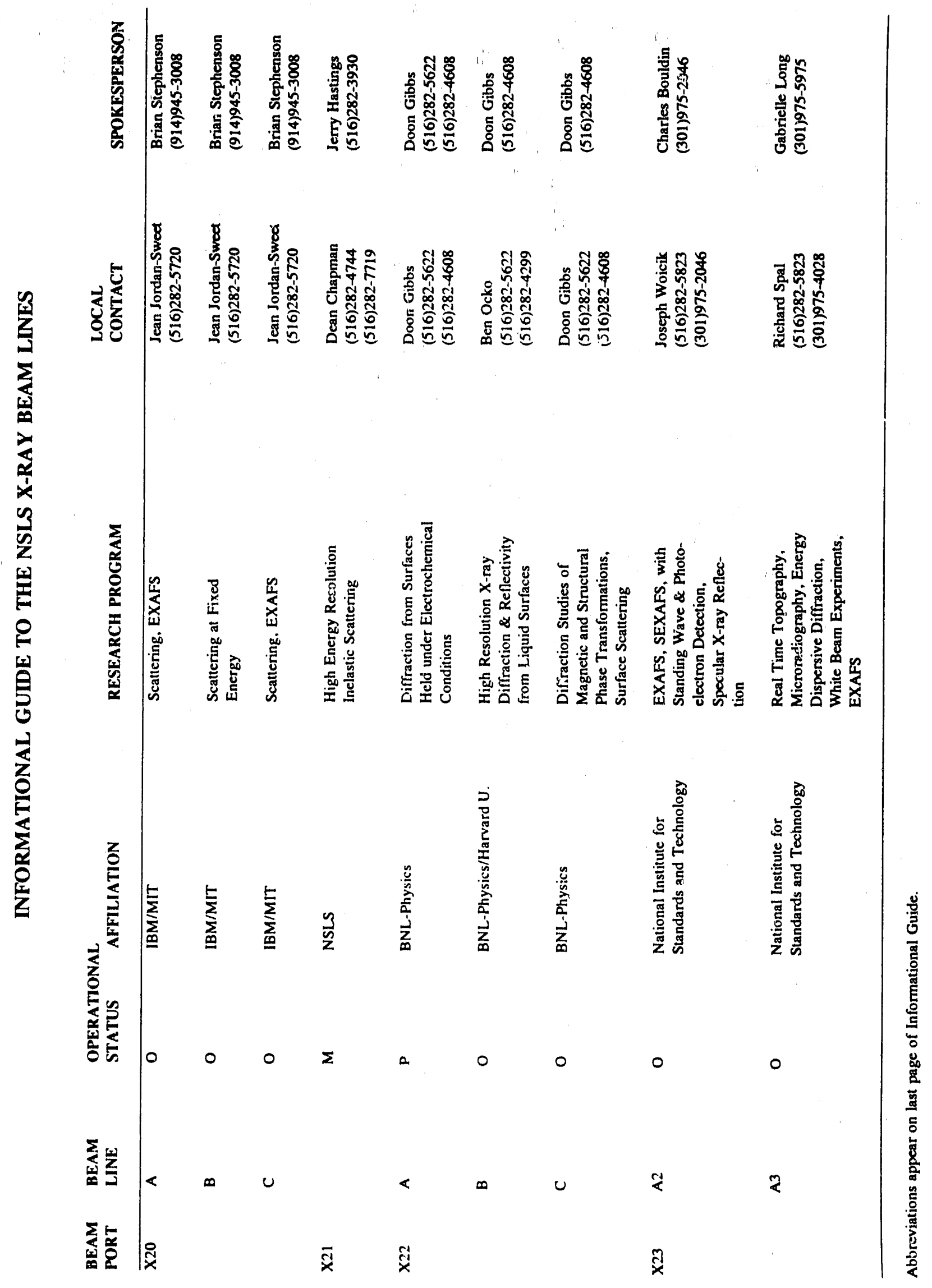




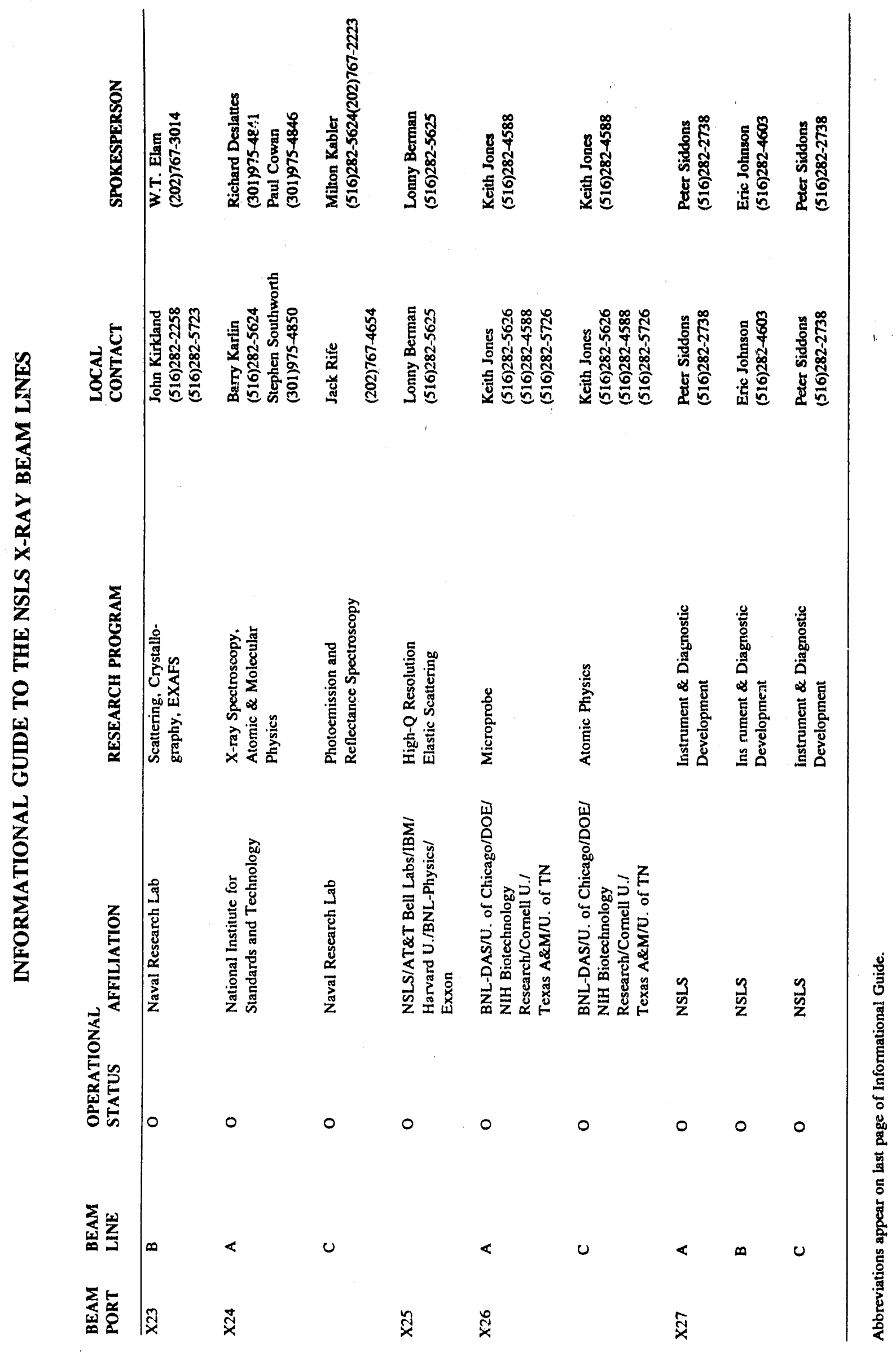




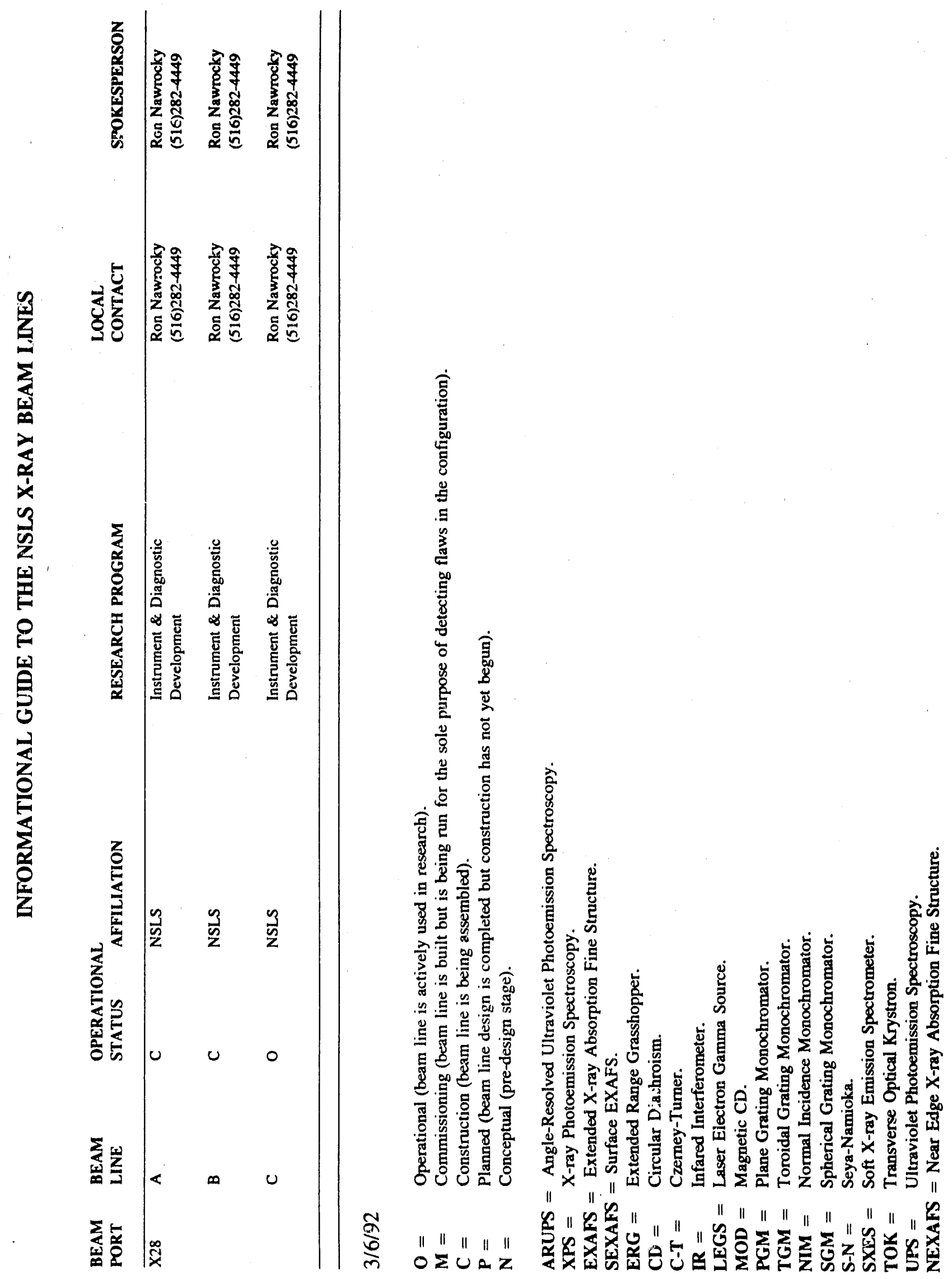



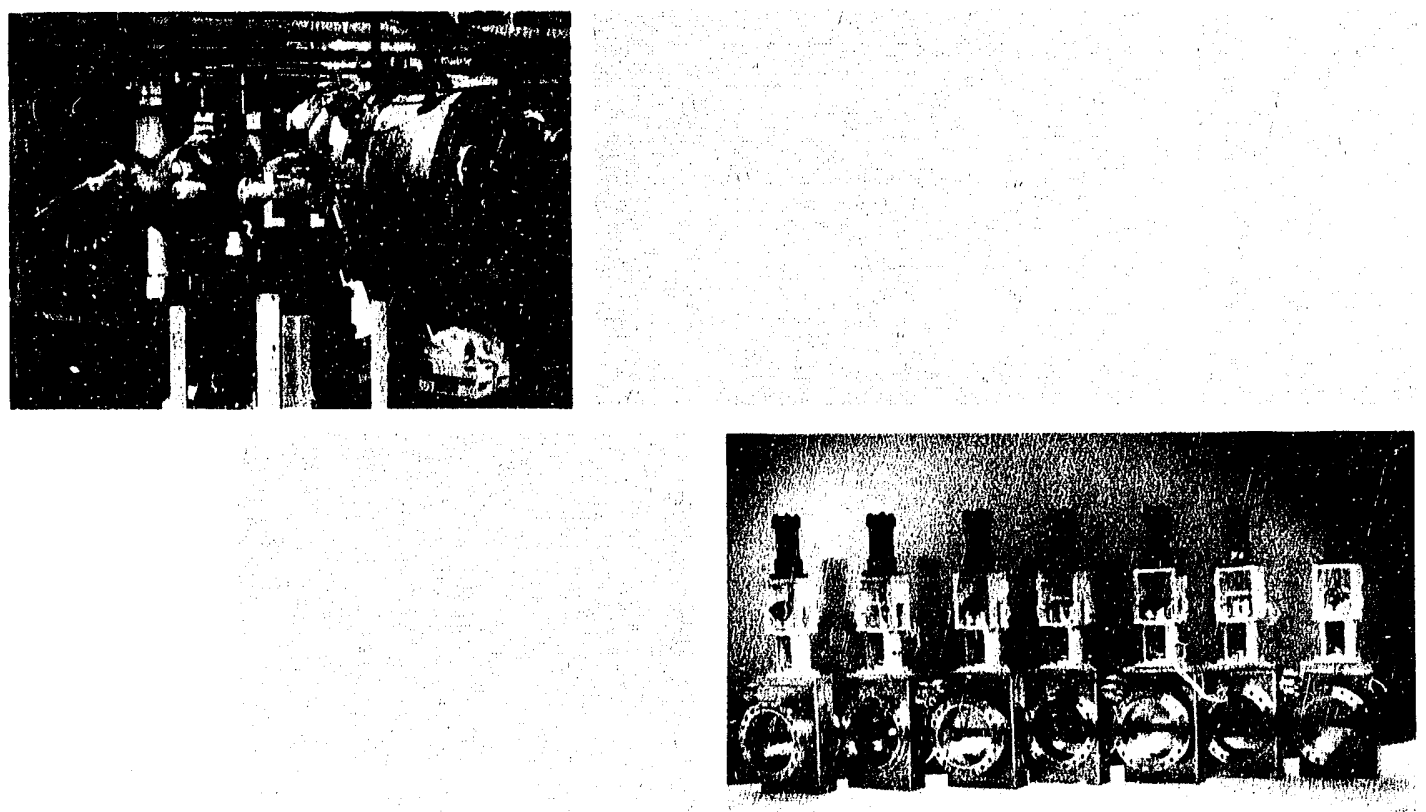

\section{Section VII Appendices}




\section{List of Abstracts: VUV Beamlines (Abstracts Located in Volume II)}

\section{Beamline U1}

Characterization of Fluorocarbon Polymer Films and Adsorbed Protein by Ultra-Soft X-ray Absorption Spectroscopy

D.G. Castner, K.B. Lewis. D.A. Fischer, B.D. Rainer, and J.L. Gland. . . . . . . . . . . . . . . . . . . .

A Fluorescence-Yield Near-Edge Spectroscopy (FYNES) Study of the Formation and Surface Reaction of NiO on $\mathrm{Ni}(100)$

J.G. Chen, J.H. Hardenbergh, R.B. Hall, and D.A. Fischer

Fluorescence Yield Oxygen $\mathrm{K}$ Edge Study of Reduced/Reoxidized $\mathrm{YBa}_{2}\left(\mathrm{Cu}_{1-\mathbf{x}} \mathrm{Fe}_{\mathbf{x}}\right)_{3} \mathrm{CuO}_{7-8}$

D.A. Fischer and A.R. Moodenbaugh.

Observation and Identification of a New Resonance in the Graphite Carbon K Near Edge Spectrum

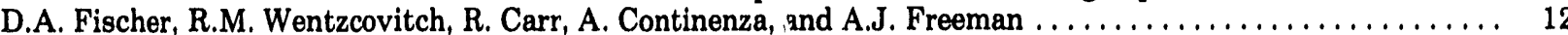

Distortions of Fluorescence Yield X-ray Absorption Spectra Due to Sample Thickness

G. Meitzner and D.A. Fischer

EXAFS Studies of Fluorine in Solid-Acid Catalysts

G. Meitzner, G.B. McVicker, and D.A. Fischer. .

Near-Edge and EXAFS Studies of Binary Alloys of 3d Metals

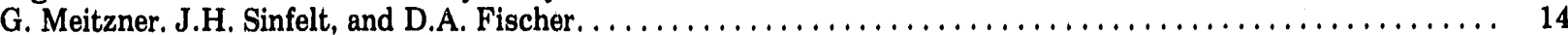

Near-Edge and EXAFS Studies of Carbon on VUV Beamline U-1

G. Meitzner, D. Cox, and D.A. Fischer.

X-ray Absorption Spectroscopic Study of New Carbon Allotrope $\mathrm{C}_{60}$, Buckminsterfullerene

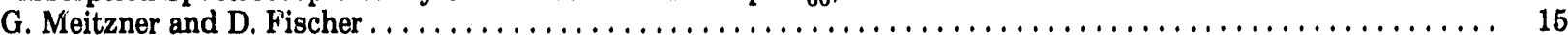

Analysis of Engine Deposits by Carbon and Oxygen Near Edge Spectroscopy

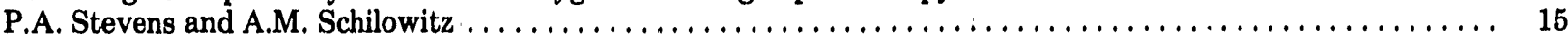

Near Edge Absorption Measurements of the Decomposition of $\mathrm{C}_{4} \mathrm{H}_{4} \mathrm{~S}$ on $\mathrm{Pt}(111)$

P. Stevens, J. Gland, T. Rufael, and A. Yeh. ..

X-ray Absorption Study of Diamond-Like Nanocomposites

X.Q. Yang, V. Dorfmann, T.A. Skotheim, D.A. Fischer, and B. Pypkin

\section{Beamline U2}

Chemically Amplified Negative Photoresit for X-ray Lithography

W. Conley, J. Gelorme, G. Spinillo, K. Stewart, and R. Wood..............................

\section{Beamline U3C}

Progress Report of Calibration Work Done on U3C

T. Archuleta, A. Bennett, R. Balke, B. Coulter, J. Cobble, R. Hockaday, T. Ortiz, S. LaMarra,

R. Montoya, E. Gullikson, S. Gallagos, J. Gonzales, P.P. Gong, and Leif Singman . .

Photoelectron Spectroscopy in Ce Heavy Fermions: Inconsistencies with the Predictions of the Prevailing

Kondo Theory

A.J. Arko, J.J. Joyce, J.M. Lawrence, R.J. Bartlett, P.C. Canfield, Z. Fisk, and J.D. Thompson. . . . . . . . . . .

Photoemission in $\mathrm{YbCu}_{2} \mathrm{Si}_{2}$ : Problems with the Kondo Model

A.J. Arko, J.J. Joyce, J.M. Lawrence, R.J. Bartlett, P.C. Canfield, Z. Fisk, and J.D. Thompson, . . . . . . . . . . .

Quasiparticle Lineshapes in $\mathrm{YBa}_{2} \mathrm{Cu}_{3} \mathrm{O}_{6.9}$

A.J. Arko, J.J. Joyce, R.J. Bartlett, R. Liu, B.W. Veal, and A.P. Paulikas . . . . . . . . . . . . . . . . . . .

Search for the Kondo Resonance in Ce Compounds

A.J. Arko, J.J. Joyce, R. Bartlett, P. Canfield, Z. Fisk, J.L. Smith, J.D. Thompson,

and J. Lawrence

Double Photoionization Studies of Argon, Neon and Helium

R.J. Bartlett, P.J. Walsh, and J. He. 
Double Photoionization Studies of He from Threshold to $560 \mathrm{eV}$

R. Bartlett, P. Walsh, J, Samson, and Z. He.

Partial Photoionization Cross Sections of Ne Across the K-Edge

R.J. Bartlett, P.J. Walsh, and J. He.

$\mathrm{Be}, \mathrm{BeO}$, and Mo Mirror Calibrations

R. Blake.

Photoemission Responses and Resonant Behavior in $\left(\mathrm{Mg}_{1-\mathrm{x}}, \mathrm{Fe}_{\mathrm{x}}\right)_{2} \mathrm{SiO}_{4}$

L.M. Hirsch and T.J. Shankland

Photoemission Study of Electronic Structure Near EF in $\mathrm{YBa}_{2} \mathrm{Cu}_{3} \mathrm{O}_{\mathrm{x}}$, for $6.3<\mathrm{x}<6.9$

R. Liu, B.W. Veal, A.P. Paulikas, J.W. Downey, A.J. Arko, and J.J. Joyce

Calibration of a Soft X-ray Film Spectrograph

T.J. Tanaka, N.D. Shinn, R.E. Ripple, A. Widman, and M.A. Palmer

ARPES Study of $\mathrm{YBa}_{2} \mathrm{Cu}_{3} \mathrm{O}_{6.7}$ and $\mathrm{YBa}_{2} \mathrm{Cu}_{3} \mathrm{O}_{6.2}$

B.W. Veal, A.P. Paulikas, R. Liu, A.J. Arko, and J.J. Joyce

ARPES Study of $\mathrm{YBa}_{2} \mathrm{Cu}_{3} \mathrm{O}_{6.9}$

B.W. Veal, A.P. Paulikas, R. Liu, A.J. Arko, and J.J. Joyce

Diffusion in Ti:Si Multilayers Probed by Anomalous Dispersion in the Soft X-ray Regime

Z. Xu, Z. Tang, S.D. Kevan, T. Novet, and D.C. Johnson .

\section{Beamline U4A}

ARP Study of the Clean and H-Covered Pt(111) Surface

W. Di, K. Smith, and S.D. Kevan.

Partial Densities of States of Cu-Pd Alloys

S.-J. Oh, M. Han, J.W. Allen, and G.K. Wertheim

Surface Atom Properties of $\mathrm{Ta}(110)$

D.M. Riffe, G.K. Wertheim, and P.H. Citrin

Vibrational Broadening of Bulk and Surface Core Levels of $\mathrm{Na}(110)$

D.M. Riffe, G.K. Wertheim, and P.H. Citrin .

Surface Bands on $\mathrm{Mo}(001)$ and $\mathrm{Mo}(001)-2 \mathrm{H}$

K.E. Smith and S.D. Kevan.

Electron Mean Free Path in the Alkali Metaly

G.K. Wertheim, D.M. Riffe, and P.H. Citrin .

Thermal Shift of Core-Electron Biuding Energies

G.K. Wertheim, D.M. Riffe, and P.H. Citrin

\section{Beamline U4B}

$\mathrm{L}_{2,3}$ Edges and Electronic Structure of Iron in Molecular Complexes

C. Cartier, P. Rudolf, A. M. Flank, and C. T. Chen.

Spin Transition Evidenced by Soft X-ray Absorption Spectroscopy

C. Cartier, P. Rudolf, A. M. Flank, and C. T. Chen.

Electronic States and Phases of $\mathrm{K}_{\mathrm{x}} \mathrm{C}_{60}$

C.T. Chen, L.H. Tjeng, P. Rudolf, G. Meigs, J.E. Rowe, J. Chen, J.P. McCauley Jr, A.B. Smith,

W.J. Romanow, and E.W. Plummer.

Studies of Y-Ba-Cu-O Single Crystals by X-ray Absorption Spectroscopy

A. Krol, Z.H. Ming, Y.H. Kao, N. Nücker, G. Roth, J. Fink, G.C. Smith, A. Erband,

G. Muller-Vogt, J. Karpinski, E. Kaldis, and K. Schönmänn.

Magnetic Moment in a Gd Iron Garnet Studied by Soft-X-ray Magnetic Circular Dichroism

P. Rudolf, F. Sette, L.H. Tjeng, G. Meigs, and C.T. Chen .

Giant $\mathrm{Cu} 2 \mathrm{p}$ Resonances in CuO Valence-Band Photoemission

L.H. Tjeng, C.T. Chen, J. Ghijsen, P. Rudolf, and F. Sette 
Soft X-ray Magnetic Circular Dichroism: A New Technique for Probing Magnetic Properties of Magnetic

Surfaces and Ultrathin Films

L.H. Tjeng, Y.U. Idzerda, P. Rudolf, F. Sette, and C.T. Chen

Soft X-ray Absorption Spectroscopy un Ni Model Compounds

J. van Elp, S.J. George, J. Chen, and S.P. Cramer

\section{Beamline U4-IR}

Pulsed Infrared Photoresponse Studies of Superconducting YBCO Films

G.L. Carr, D.B. Romero, D.B. Tanner, S. Etemad, F. DeRosa, and A. Inam. . . . . . . . . . . . . . . . . . . . 32

Time-Resolved IR Studies of Photo-Induced States in High- $\mathrm{T}_{\mathrm{c}}$ Superconductors

G.L. Carr, D.B. Romero, D.B. Tanner, S. Etemad, A. Inam, W. O'Brien, D. Ederer, C.J. Hirschmugl,

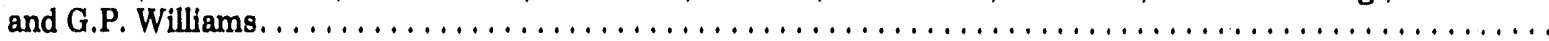

Potassium Enhanced Oxidation of $\mathrm{Cu}(100)$

F.M. Hoffman, K. Lin, R. Tobin, P. Dumas, G.P. Williams, and C.H. Hirschmugl. . . . . . . . . . . . . . 33

Infrared Transmission of Superconducting Niobium Films

C. Kendziora, D. Mandrus, D. Koller, S. Gausepohl, L. Mihaly, C. Hirschmugl, and G. Williams ... . . . . . . 34

Far-IR Study of Adsorbates on $\mathrm{Cu}(100)$

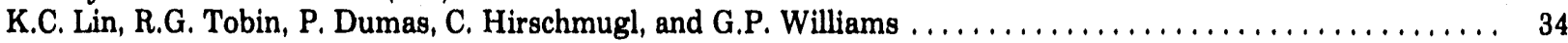

Far Infrared Measurements of the Absorption of Ammonia Using a Mach-Zender Interferometer

K.D. Moeller, T. Sears, H.T. Liu, C.J. Hirschmugl, and G.P. Williams . . . . . . . . . . . . . . . . .

\section{Beamline U5}

Spin Polarized Photoemission Studies of Surface and Interfacial Magnetism

N.B. Brookes, Y. Chang, A. Clarke, P.D. Johnson, and M. Weinert

Studies of $\mathrm{K}$ on $\mathrm{Fe}(001)$ with Spin Polarized Photoemission

Y. Chang, N.B. Brookes, and P.D. Johnson.

Spin-Polarized Photoemission Study of the $\mathrm{Pd} / \mathrm{Fe}(100) \mathrm{I}$ iterface

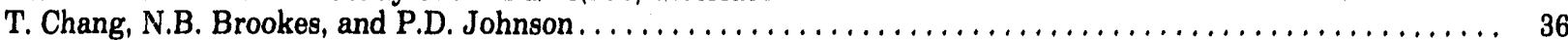

Local Spin Correlations in Ultra-Thin Fe/W(100) Films

G.A. Mulhollan, R.L. Fink, J.L. Erskine, and G.K. Walters

\section{Beamline U6}

X-ray Mask Distortion Due to Radiation Damage

R.E. Acosta.

$\mathrm{X}$-ray Radiation Damage and a Reliability Study on Bipolar Devices

D. Boyne, L. Hsia, R. Wachnik, R. Decker, and C. Wasik

Chemically Amplified Negative Photoresist for X-ray Lithography

W. Conley, J. Gelorme, G. Spinillo, K. Stewart, and R. Wood

Effect of Synchrotron X-ray Radiation on the Channel Hot-Carrier Reliability of Reoxidized Nitrided

Silicon Dioxide

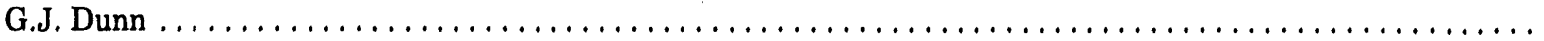

A Study of X-ray Damage Effects in Open-Bottom Trench Isolation for Bipolar Devices

L.C. Hsia

A Search for Neutral Electron Traps in Synchrotron Irradiated Silicon/Silicon Dioxide Structures

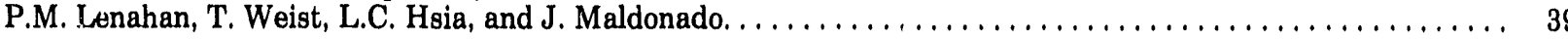

X-ray Mask Repair

A. Wagner, J.P. Levin, J.L. Mauer, P.G. Blauner, S.J. Kirch, P. Longo, and J.P. Silverman . . . . . . . . . . 40

\section{Beamline U7A}

The Condensation and Photon Decomposition of Diborane

M.W. Ruckman, J. Mowlem, and D. Strongin 
Photon Assisted Formation of Diamond-Like Carbon Films from Organics

M.W. Ruckman, J. Mowlem, and D. Strongin.

Reaction of Potassium with Ammonia at Liquid Nitrogen Temperatures

M. Ruckman, E. Horache, and M. Strongin.

Photon-Assisted Reaction of Diborane and Ammonia to Form Boron Nitride

D. Strongin, J. Mowlem, M.W. Ruckman, and M. Strongin

\section{Beamline U7B}

Valence States in Thin Eu-Rh and Eu-Pd Films

C. Berg, S. Raaen, and M.W. Ruckman .

Photon Modification of Aluminum Oxide on 6063 Aluminum

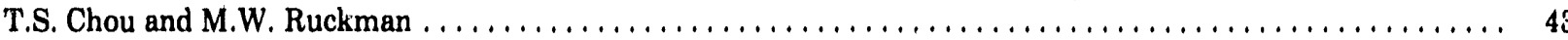

Photoelectron Spectroscopic Studies of the Electronic Structure of Ferrous Sulfide

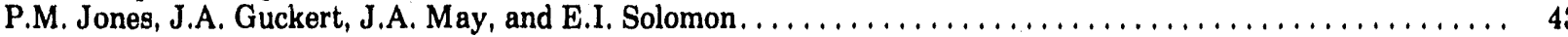

Polarization Dependence of X-ray Absorption in $\mathrm{O}_{2}$; the Square Well Model

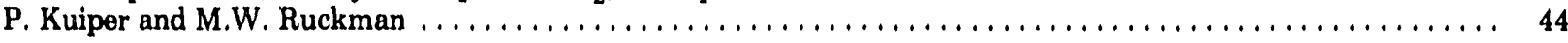

Spin-Resolved XAS of $\mathrm{O}_{2}$

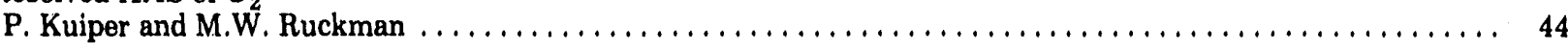

Valence Density of States of Hydrogen-Reduced NiO(100)

M.A. Langell, K.W. Wulser, and B.P. Hearty .

Electronic Properties of Body-Centered-Tetragonal Copper

H. Li, S.C. Wu, J. Quinn, Y.S. Li, D. Tian, and F. Jona .

Structural and Electronic Properties of Ultrathin Films of Gd, Tb, Dy, Ho and Er

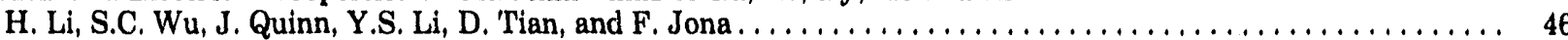

Ultra-Thin Films of $\mathrm{Rh}$ on $\mathrm{Au}(001)$ and $\mathrm{Rh}$ on $\mathrm{Ag}(001)$ : Growth Mode and Magnetism

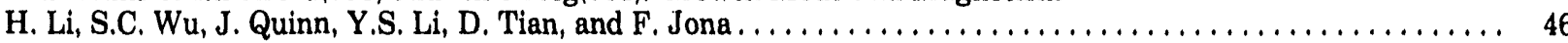

Core Level Lineshapes in Fe-V Alloys

S.L. Qiu, R.G. Jordan, M.W. Ruckman, and M. Strongin

Partial Densities of States in CuAuI

S.L. Qiu, R.G. Jordan, M.W. Ruckman, and M. Strongin

Atomic and Electronic Structure of Thin Films of Mn on $\operatorname{Pd}\{111\}$

D. Tian, H. Li, S.C. Wu, F. Jona, and P.M. Marcus

Re-Examination of the Electron Band Structure of $\mathrm{Tb}$ Along $\Gamma \Delta \mathrm{A}$

S.C. Wu, H. Li, Y.S. Li, D. Tian, J. Quinn, F. Jona, D. Fort, and N.E. Christensen . . . . . . . . . . . . 48

Relativistic Effects in the Electron Band Structure of $\mathrm{Cu}\{111\}$

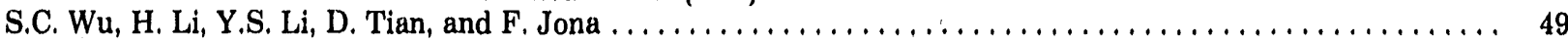

Surface State on Clean $\mathrm{Tb}(0001)$

S.C. Wu, H. Li, Y.S. Li, D. Tian, J. Quinn, and F. Jona . . . . . . . . . . . . . . . . . . . . . . . .

\section{Beamline U8A/B}

Chlorine Adsorption on Room Temperature GaAs(110)

D.K. Shuh and J.A. Yarmoff.

Unfilled Orbitals of $\mathrm{C}_{60}$ and $\mathrm{C}_{70}$ from Carbon K-Shell X-ray Absorption Fine Structure

L.J. Terminello, D.K. Shuh, F.J. Himpsel, D.A. Lapiano-Smith, J. Stöhr, D.S. Bethune,

and G. Meijer

Photoelectron Spectroscopy Study of the $\mathrm{Cr} / \mathrm{MoS}_{2}$ Interface

T.D. Durbin, D.K. Shuh, J.A. Yarmoff, J.R. Lince, and S.V. Didziulis.

Photoemission Studies of the Chemisorption of Dichlorosilane on the $\mathrm{Si}(111)$ and $\mathrm{Si}(100)$ Surfaces

T.D. Durbin, D.K. Shuh, J.A. Yarmoff, D.A. Lapiano-Smith, F.J. Himpsel, and F.R. McFeely . . . . . . . . . . . 5

The Decomposition of $\mathrm{BCl}_{3}$ on $\mathrm{Si}(111)$ and the Subsequent Incorporation of Boron into Silicon

D.A. Lapiano-Smith and F.R. McFeely 
A Mechanism for Diborane Induced Selectivity Loss in the CVD of Silicon from $\mathrm{SiH}_{2} \mathrm{Cl}_{2}$

D.A. Lapiano-Smith and F.R. McFeely

Studies of the $\mathrm{XeF}_{2}$ Etching of $\mathrm{Si}(111)$ at $200^{\circ} \mathrm{C}$

C.W. Lo, D.K. Shuh, and J.A. Yarmoff

Construction and Launch of an Improved Soft X-ray Telescope

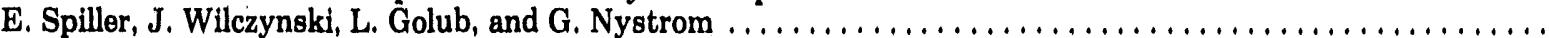

\section{Beamline U9B}

Structural Analysis of Phage T7 Gene 5.9 Product, Using Circular Dichroism Spectroscopy and Primary

Sequence Analyses

L.L. France, L. Lin, B. Studier, and J.C. Sutherland

Structural Analysis of Variable Membrane Protein 7 from Borelia HermsII, Using Circular Dichroism Spectroscopy

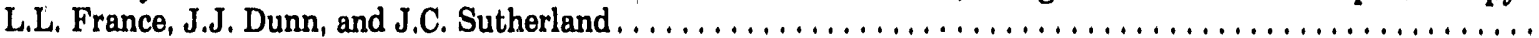

A Vacuum UV CD Study of Pyrophosphatase Wild Type and Mutants

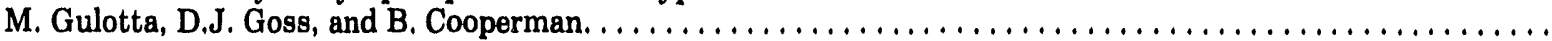

The Vacuum UV CD Spectrum of $A \mathrm{G} \cdot \mathrm{G} \cdot \mathrm{C}$ Triplex

K.H. Johnson, M.E. Hogan, and J.C. Sutherland.

Circular Dichroism Study of an Oligodeoxyribonucleotide Triplex

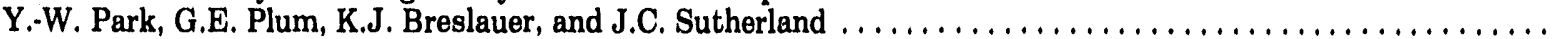

\section{Beamline U10A}

Investigation of the Al L Exciton in AlGaAs by Soft X-ray Reflection and Emission

Q.-Y. Dong, W.L. O'Brien, J.J. Jia, T.A. Callcott, D.R. Mueller, and D.L. Ederer . . . . . . . . . . . . . . . . 56

Copper MII,III X-ray Emission Spectra for $\mathrm{CuO}, \mathrm{Cu}_{2} \mathrm{O}$ and $\mathrm{Bi}_{2} \mathrm{Sr}_{2} \mathrm{CaCu}_{2} \mathrm{O}_{8}$

D.R. Mueller, D.L. Ederer, J.J. Jia, W.L. O'Brien, Q.Y. Dong, and T.A. Callcott. . . . . . . . . . . . . . . . 57

The NIV,V $\rightarrow$ OII,III Soft X-ray Emission Spectrum of La

D.R. Mueller, C.W. Clark, D.L. Ederer, J.J. Jia, W.L. O'Brien, Q.Y. Dong, and T.A. Callcott . . . . . . . . . . .

Electronic Structure of $\mathrm{MgO}, \alpha \cdot \mathrm{Al}_{2} \mathrm{O}_{3}$ and $\mathrm{MgAl}_{2} \mathrm{O}_{4}$, Occupied and Unoccupied Bulk DOS

W.L. O'Brien, J. Jia, Q.Y.Y. Dong, T.A. Callcott, D.T. Mueller, and D.L. Ederer

Phonon Coupling to Core Excitations in Ionic Solids

W.L. O'Brien, J.Jia, Q.-Y. Dong, T.A. Callcott, D.R. Mueller, and D.L. Ederer

\section{Beamline U11}

Photon-Induced Chemistry in Weakly-Bound Thiophene Clusters

P. Cid-Aguero, E.A. Walters, and J.R. Grover

Photoionization Studies of Trifluoromethyl Bromide and a Cluster with Methanol

J.T. Clay, E.A. Walters, M.V. Willcox, and J.R. Grover

Complexes of Oxygen with Benzene and Hexafluorubenzene

J.R. Grover, G. Hagenow, and E.A. Walters

Kinetic Energy Release in the Dissociative Photoionization of Van Der Waals Clusters

J.R. Grover, E.A. Walters, D.L. Arneberg, G. Hagenow, J.T. Clay, and M.V. Willcox

Photoionization Mass Spectrometric Study of N $+\mathrm{C}_{2} \mathrm{H}_{5}$ Reaction Products

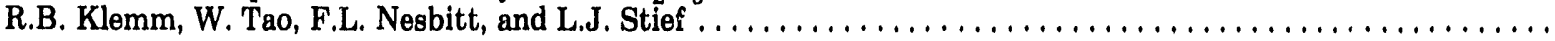

Photnionization Spectra and Ionization Thresholds for $\mathrm{H}_{2} \mathrm{CN}$ and $\mathrm{D}_{2} \mathrm{CN}$

F.L. Nesbitt, G. Marston, L.J. Stief, M.A. Wickramaaratchi, W. Tao, and R.B. Klemm . . . . . . . . . . . . . .

A Possible Role for Ions in Flame Extinguishment by Halons

E.A. Walters, J.R. Grover, J.T. Clay, M.V. Willcox, D.L. Arneberg, and J.S. Ninitz . . . . . . . . . . . . . . . . .

\section{Beamline U12B}

Coverage Dependence of the Li K-Edge Absorption for $\mathrm{Li} / \mathrm{Be}(11 \overline{2} 0)$

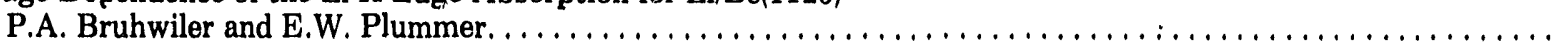


Angle-Resolved Photoemission Investigation of Bi Overlayers on InSb(110) Surfaces

The Experimental Band Structure of $\mathrm{Be}(11 \underline{2} 0)$

J.B. Hannon, G. Lee, and E.W. Plummer

Core-Level Spectrtoscrpy from Low-Index Surfaces of $\mathrm{TaC}$

S.C. Lui, G.R. Cruzalski, D. Heskett, X. Shi, and D.M. Zehner . . . . . . . . . . . . . . . . . . . . .

Interantion of Hydrogen with $\mathrm{Mg}(0001)$

P.T. Sprunger, P.A. Bruhwiler, and E.W. Plummer .

Bulk Rand Structure of Lithium

G.M. Watson, X.Y. Liu, and E.W. Plummer .

\section{Beamline U13UA}

Core Electron Excitation of Simple Molecules to Rydberg States - A New Method to See Hidden Resonance

Excitations

D.Y. Kim, K. Lee, C.-I. Ma, M. Mahalingham, D.M. Hanson, and S.L. Hulbert . . . . . . . . . . . . . . . . .

Selective Excitation of Carbon in Acetone

C.-I. Ma, K. Lee, D.Y Kim, D.M. Hanson, and S. Hulbert.

\section{Beamline U13UB}

$\mathrm{X}$-ray Imaging and Projection Lithography

J.E. Bjorkholm, J. Bokor, R.M. D'Souza, L. Eichner, R.R. Freeman, T.E. Jewell, W.M. Mansfield,

A.A. MacDowell, E.L. Raab, L.H. Szeto, D.M. Tennant, W.K. Waskiewicz, D.L. White, D.L. Windt,

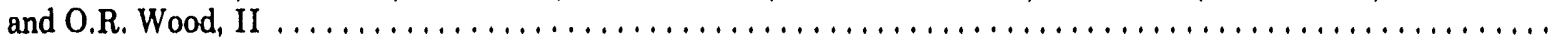

Soft X-ray Projection Lithography

R. Freeman, J. Bjorkholm, J, Bokor, K. Early, L. Eichner, M. Himel, T. Jewell, A. MacDowell,

L. Szeto, D. Taylor, D. Tennant, W. Waskiewicz, D. White, D. Windt, O. Wood, II, P. Takacs,

J. Bruning, M. Ruda, and K. Thompson

\section{Beamline U14A}

Line Shape Of The Si $\mathrm{L}_{2,3}$ VV Auger Spectrum of Stoichiometric $\mathrm{SiO}_{2}$ Measured by APErS

Bartynski, R. A., See, A. K., Kao, C.-C., and Hulbert, S.L.

Electronic Structure of the $\mathrm{SiO}_{2} / \mathrm{Si}(111)$ Interface Probed by APECS

See, A.K., Bartynski, R.A., Ke.o, C.-C., Hulbert, S.L.

Photoemission Investigation of the Core Levels of $\mathrm{Na} / \mathrm{Cu}(111)$

X. Shi, D. Tang, D. Heskett, and K.-D. Tsuei .

\section{Beamline U15}

Fragmentation of Gaseous Freons: Electron-Multiple-Ion Coincidence Measurements

T.A. Ferrett, S. Hsieh, M. Bayne, D.Y. Kim, K. Lee, C. I. Ma, and D.M. Hanson . . . . . . . . . . . . . . . . .

Electron-Ion Coincidence Mass Spectra of $\mathrm{CO}$

R.G. Hayes, K. Lee, C.I. Ma, and D.M. Hanson

Electron-Ion Coincidence Mass Spectra of $\mathrm{NH}_{3}$

R.G. Hayes, K. Lee, C.I. Ma, and D.M. Hanson

Electron-Ion Coincidence Studies of the Behavior of Ammonia Upon Core Electron Excitation

R.G.'Hayes, K. Lee, C.-I. Ma, and D.M. Hanson.

Electron-Ion Coincidence Studies of the Behavior of Methylamine Upon Core Electron Excitation

R.G. Hayes, K. Lee, C.-I. Ma, and D.M. Hanson.

The Anisotropic Fragmentation of $\mathrm{CO}_{2}, \mathrm{~N}_{2} \mathrm{O}$, and $\mathrm{H}_{2} \mathrm{O}$ Following Core Electron Excitation

D.Y. Kim, K. Lee, C.I. Ma, and D.M. Hanson 
Local Environment Around the Oxygen Atoms in Y-Ba-Cu-O Studied by X-ray Absorption Fine Structure (X A F' S) Spectroscopy

A. Krol, Z.H. Ming, Y.H. Kao, N. Nücker, G, Roth, J, Fink, and G.C. Smith . . . . . . . . . . . . . . . . .

Local Structure Around $\mathrm{F}$ in $\mathrm{Nd}_{2} \mathrm{CuO}_{4-\mathrm{x}} \mathrm{F}_{\mathrm{x}}$

A. Krol, Y.L. Soo, Z.H. Ming, S. Huang, Y.H. Kao, G.C. Smith, K, Lee, A.C.W.P. James,

and D.W. Murphy

Angular Dependence of Photofragments from K-Shell Excited Diatomics

K. Lev, C.I. Ma, D.Y. Kim, and D.M. Hanson.

Determination of the Harmonic Composition of the Synchrotron Radiation at U15

K. Lee, D.M. Hanson, and Y.H. Kao .

Electron-Multiple-Ion Coincidence Studies of Methanol

C.-I. Ma, K. Lee, D.Y. Kim, and D.M. Hanson

\section{Beamline U16A}

Spin-Polarized Angle-Resolved Photoemission Studies of Ultra-Thin Fe/W(110) Films

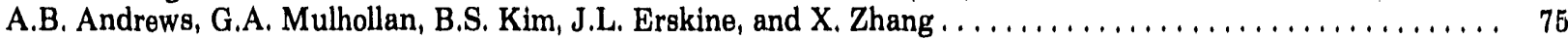

ARUPS Study of Bulk Gd(0001)

B. Kim, A.B. Andrews, J.L. Erskine, K. Kim, and B.N. Harmon . . . . . . . . . . . . . . . . . . . 75

ARUPS Temperature Dependence of RKKY Induced Magnetic Splitting in Bulk Gd(0001)

B. Kim, A.B. Androws, J.L. Erskine, K. Kim, and B.N. Harmon . . . . . . . . . . . . . . . . . . . . . 76

High-Resolution Core-Level Spectroscopy of Epitaxial Bi-Metallic Interfaces

B. Kim, N.D. Shinn, A.B. Andrews, and J.L. Erskine

Angle-Resolved Photoemission Evidence for a Gd(0001) Surface State

D. Li, C.W. Hutchings, P.A. Dowben, C. Hwang, R.-T. Wu, M. Onellion, B. Andrews,

and J.L. Erskine.

Temperature Dependence of Core-Level Binding Energies of $\mathrm{W}(110)$ and $\mathrm{Ta}(100)$

D.M. Riffe, W. Hale, B.S. Kim, and J.L. Erskine

\section{Beamline U16B}

The Influence of Preadsorbed Potassium on the Adsorption of $\mathrm{PF}_{3}$ on $\mathrm{Ru}(0001)$ at $\mathrm{LN}_{2}$ Temperatures

V. Chakarian, D.K. Shuh, J.A. Yarmoff, H.S. Tao, B. Maschhoff, and T.E. Madey . . . . . . . . . . . . . . . 78

Chemical Bonding and Structure in Epitaxial Aluminas(III): Analysis of Alumina Valence Band Spectra

R.P. Merrill, T.N. Rhodin, S. Woronick, P. O'Hagen, N.D. Shinn, R. Absil, and A. Chester . . . . . . . . . . . . 78

X-ray Spectroscopy of Catalytic Materials: Surface Acidity in Epitaxial Alumina and the Effect of Heat Treatment on Ethylene Adsorption

R.P. Merrill, T.N, Rhodin, S. Woronick, P. O'Hagen, N.D. Shinn, G. Woolery, R. Absil,

and $A$. Chester

Decay Channels of $\mathrm{Al} \mathrm{L}_{2}{ }_{3}$ Excitons and Absence of $\mathrm{O} \mathrm{K}$ Excitons in $\alpha \cdot \mathrm{Al}_{2} \mathrm{O}_{3}$

W.L. O'Brien, J. Jia, Q.Y. Dong, T.A. Callcott, D.L. Mueller, D.L. Ederer, N.D. Shinn,

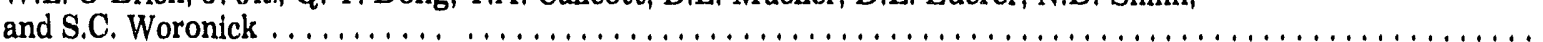

Oxidation of W(110): W(4f) Core-Level Spectroscopy

C.H.F. Peden and N.D. Shinn

The Structure of Thermally Nitrided Si $(100)$

C.H.F. Peden, J.W. Rogers, Jr., N.D. Shinn, K.B. Kidd, and K.L. Tsang 


\section{List of Abstracts: X-ray Beamlines (Abstracts Located in Volume II)}

\section{Beamline X1A}

Improved Images with the Scanning Photoemission Microscope

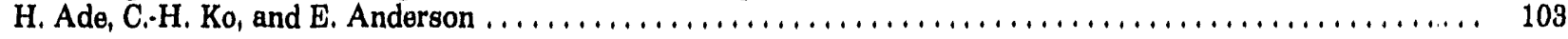

Photon Beam Mediated Oxidation on Aluminum Surfaces

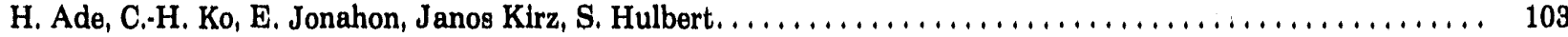

Investigation of Calcium Deposits in Human Cartilage Using STXM

C.J. Buckley, G.F. Foster, R.E. Y.S. All, C. Scotchford, and J. Kirz . . . . . . . . . . . . . . . . . . . . . 104

Imaging of Wet, Unfixed and Unstained Muscle at 50nm Resolution

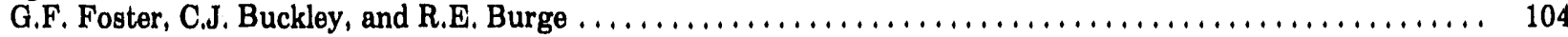

Quantitative Measurements of Protein Flux Across the Zymogen Granule Membrane Using STXM

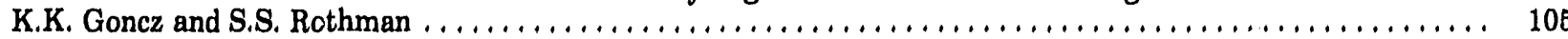

Diffraction-Limited Imaging in a Scanning Transmission X-ray Miscroscope C. Jacobsen, S. Williams, J. Kirz, X. Zhang, E. Anderson, M.T. Browne, C.J. Buckley, D. Kern,

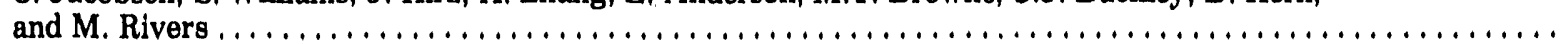

Scanning Luminescence X-ray Microscopy C. Jacobsen, S. Lindaas, S. Williams, and X. Zhang.

X-ray Holography Using Photoresists C. Jacobsen, S. Lindaas, and M. Howells.

Differential Phase Contrast Imaging with the STXM

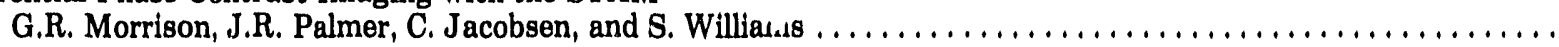

\section{Beamline X1B}

Screening Power of Spectator Electrons: Observation of the Electronic Decay of Core Hole-ilydberg Electron States in $\mathrm{N}_{2}$

W. Eberhardt, J-E. Rubensson, K.J. Randall, J. Feldhaus, A.L.D. Kilcoyne, A.M. Bradshaw,

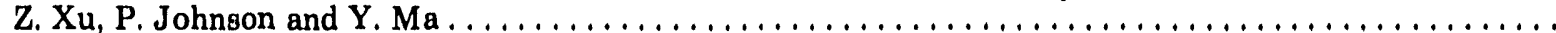

Soft X-ray Spectroscopy at the X1 Undulator K.J. Randall, J. Feldhaus, W. Erlebach, A.M. Bradshaw, W. Eberhardt, Z. Xu, P.D. Johnson,

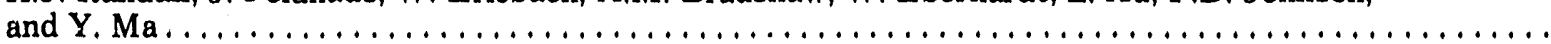

Core Level Photoelectron Spectroscopy of Gas Phase Molecules at Vibrational Resolution

K.J. Randall, J. Feldhaus, A.L.D. Kilcoyne, A.M. Bradshaw, W. Eberhardt, J-E. Rubensson,

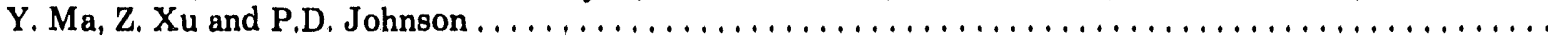

Decay of Vibrationally Selected Core Hole States in Small Molecules J-E. Rubensson, W. Eberhardt, K.J. Randall, J. Feldhaus, A.L.D. Kilcoyne, A.M. Bradshaw,

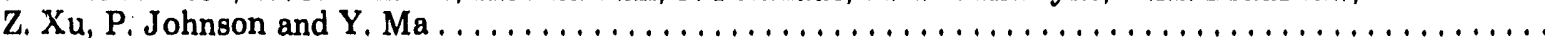

\section{Beamline X2}

Microtomography of Calcium Deposits in Cartilage Tissue

K.L. D'Ámico, J.H. Dunsmuir, S.R. Ferguson and C.J. Buckley

Determination of the Spatial Distribution of Metal Atoms in a Supported Catalyst System

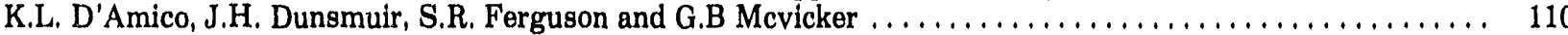

Observation of the Settling of Particulates in a Heavy Oil

J.H. Dunsmuir, K.L. D'Amico and S.R. Ferguson

\section{Beamline X3A2}

Phase Determination for Macromolecular Crystals Using X-ray Multiple Diffraction S.L. Chang, H. King, M.T. Huang and Y. Gao 
Phase Separation Kinetics of a Segmented Polyurethane

Vol II

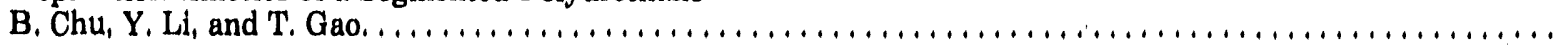

Synchrotron SAXS Study of Mean.Field and Ising-like Critical Behavior of Poly(2-chloro-styrene)/Polystyrene Blends B. Chu, Q. Ying, K. Linliu, P. Xie, T, Gao, Y, Li, T, Nose, M. Okada, A. Darovsky,

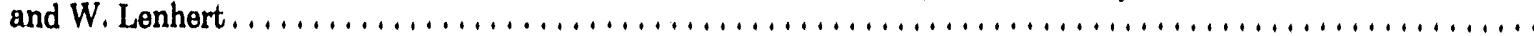

Structural Changes Upon Electrochemical Insertion of Lithium in the 2212 BiSrCuO Superconducting Phase

P. Coppens, P. Lee, Y. Gao, N.A. Fleischer, and J, Manassen. . . . . . . . . . . . . . . . . . . . . . . .

Lattice Instabilities and the Effect of Copper-Oxygen Sheet Distortions on Superconductivity in

$\mathrm{La}_{2} \mathrm{CuO}_{4}$-Superconductors: $\mathrm{La}_{1,60-x} \mathrm{Nd}_{0,40} \mathrm{Sr}_{\mathrm{x}} \mathrm{CuO}_{4}$

M. Crawford, R. Harlow, E. McCarron, and W. Farneth

Morphology Studies of CHDI Urethane Elastomers by X-ray Diffraction Using Synchrotron Radiation

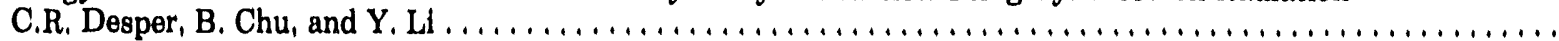

SAXS and WAXS Study of Polyurethanes Containing Polyethylene Soft Segments

C.R. Desper, B. Chu, T. Gao, Y. Li, and C.A. Byrne.

Single Crystal Anomalous Scattering Studies of Mixed.Valence Complexes

A. Frost-Jensen, M. Pressprich, Y. Gao, and P. Coppens. . . . . . . . . . . . . . . . . . . . . . . .

Lattice Instabilities in $\mathrm{Nd}$-Doped $\mathrm{La}_{2} \mathrm{CuO}_{4}$; Fresence of a PCCN Phase?

R. Harlow, M. Crawford, E. McCarron, and W. Farneth

Lattice Instabilities and the Effect of Copper-Oxygen Sheet Distortions on Superconductivity in

$\mathrm{Lib}_{2} \mathrm{CuO}_{4}$-Superconductors: $\mathrm{La}_{1,88-x} \mathrm{Nd}_{\mathrm{x}} \mathrm{Sr}_{0,12} \mathrm{CuO}_{4}$

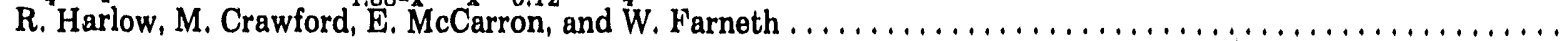

Crystallization and Melting Study of Poly(Aryl Ether Ketones)

B. Hsiao, K. Gardner, D. Wu, and B. Chu

Time-Resolved Synchrotron Study of Poly(Aryl Ether Ketones): Crystallization and Melting

B. Hsiao, K. Gardner, D. Wu, and B. Chu

Crystallization and Melting

Thermally.Induced Structural Changes in a Segmented Polyurethane

Y. Li, J. Liu, B. Chu, A. Darovsky, and W. Lenhert.

Protein Crystals tor Phv'sical Estimation of X-ray Triplet Phases

F. Mo, B.C. Hauback, E. Husstad, M. Teeter, L.C. Sieker, and M.S. Lehmann . . . . . . . . . . . . . . . . . . .

Structures of Alkali-Doped Fullerness by Powder Diffraction

P.W. Stephens, L. Mihaly, F. Diederich, K. Holczer, S.-M. Huang, R.B. Kaner, R.L. Whetten,

J.B. Wiley, and P.L. Lee . . . . . . . . . . . . . . . . . . . . . . . . . . . . . . . . . . . . . . .

Time-Resolved X-ray Scattering Studies of the Crystallization of Polyethylene Blends

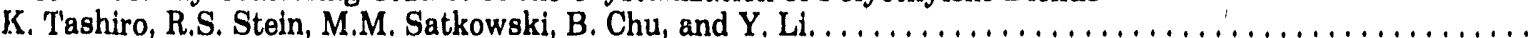

Determination of the Structural Distortions Corresponding to the ${ }_{\mathrm{q} 1}$-Type and $\mathrm{q}_{2}$-Type Modulations in Niobium

Trisigleride

S. van Smaalen, J.L. deBoer, A. Meetsma, H. Graafsma, H.-S. Sheu A. Darovskikh,

and P. Coplens.

Temperature Effect on the Small Angle X-ray Scattering of an Ionomer

J. Wang, Y. Li, D.G. Peiffer, R.D. Lundberg, and B. Chu .

Srrall-Angle X-ray Scattering from Microvoids in Amorphous Silicon-Based Semiconductors

D.T. Williamson and Y. Chen

\section{Beamline X3B1}

Investigation of Microstructure of Interfaces in $\mathrm{Si}_{1} / \mathrm{Ge}_{0.2} \mathrm{Si}_{0.8}$ Superlattices by Means of X-ray Reflectivity and and $X \cdot$ ray Standing Wave

Z.H. Ming, A. Krol, Y.L. Soo, Y.H. Kao, and K.L. Wang

\section{Beamline X5}

The Tensor Force Between Quarks and the Deformation of Baryons

G. Blanpied, M. Blecher, A. Caracappa, C. Djalali, M-A. Duval, G. Giordano, S. Hoblit, M. Khandaker,

C. Kistner, G. Matone, L. Miceli, W. K. Mize, B.M. Preedom, A.M. Sandorfi, C. Schaerf, R.M. Sealock,

C.E. Thorn, S.T. Thornton, K. Vaziri, C.S. Whisnant, X. Zhao, and M.A. Moinester . . . . . . . . . . . . . . . . . 
Beamline X6B

Anomalous X-ray Reflectivity Measurements on Ni/Fe Alloy Thin Filims

J.M. Bai, P.A. Montano, and M. Tomkiewicz .

Glaricing Angle X-ray Fluoresence Study of Ni/W Multilayers

J.M. Bai, K. Huang, P.A. Montano, M. Grimsditch, E. Fullerton, D. Kelly, and I.K. Schuller . . . . . . . . . . . . 122

In Situ X-ray Diffraction of the Early Stages of the Crystallization of $\mathrm{Fe}_{80} \mathrm{~B}_{20}$

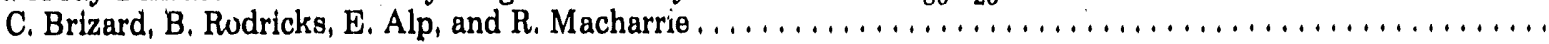

X-ray Reflectivity Studies of Diblock Copolymer Films

Z. Cai, G.P. Felcher, K. Huang, P.A. Montano, J.M. Bai, T.P. Russell, and G.W. Zajac . ................

In-Situ X-ray Reflectivity Study of Sputter Deposition

R.P. Chiarello, H.K. Kim, and H, You

Interface Structure of Epitaxial Si/CoSi $/$ Sil(001): an X-ray Reflectivity and Fluoresence Study

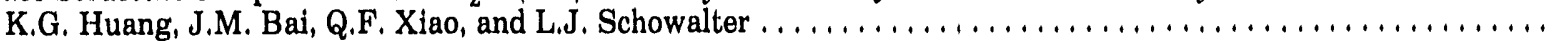

\section{Beamline X7A}

Structural Investigations of Some Synthetic Zeolite Phases

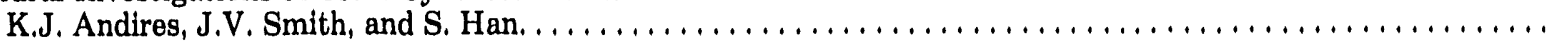

Lattice Instabilities and the Effect of Copper-Oxygen Sheet Distortions on Superconductivity in

$\mathrm{La}_{2} \mathrm{CuO}_{4}$-Superconductors: $\mathrm{La}_{1,60-x} \mathrm{Nd}_{0,40} \mathrm{Sr}_{\mathrm{x}} \mathrm{CuO}_{4}$

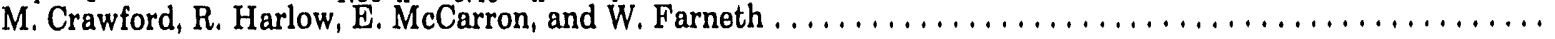

In Situ Powder Diffraction Studies of the Hydriding Behavior of Nanocrystalline Pd

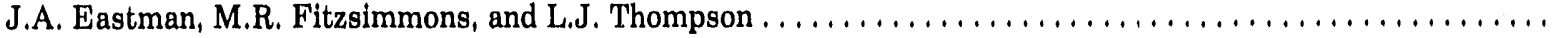

Lattice Instabilities in $\mathrm{Nd}$-Doped $\mathrm{La}_{2} \mathrm{CuO}_{4}$ : Presence of a PCCN Phase?

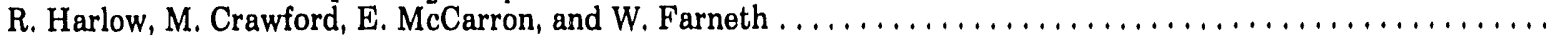

Lattice Instabilities and the Effect of Copper-Oxygen Sheet Distortions on Superconductivity in:

$\mathrm{La}_{2} \mathrm{CuO}_{4}$-Superconductors: $\mathrm{La}_{1,88-\mathrm{x}} \mathrm{Nd}_{\mathrm{x}} \mathrm{Sr}_{0.12} \mathrm{CuO}_{4}$

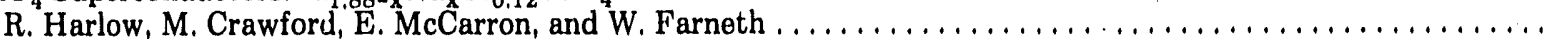

Orientational Ordering Phase Transition in $\mathrm{C}_{60}$

P.A. Heiney, J.E. Fischer, A.R. McGhie, W.J. Romanow A.N. Denenstein, J.P. McCauley, Jr., A.B. Smith,

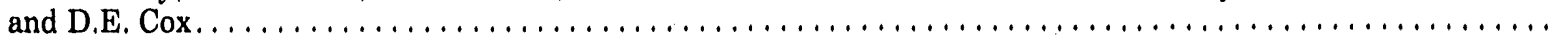

The Crystal Structure Determination of $\mathrm{BiMo}_{2} \mathrm{O}_{7}(\mathrm{OH}) \cdot 2 \mathrm{H}_{2} \mathrm{O}$ from Powder Diffraction Data

J.A. Hriljac and C.C. Torardi . . . . . . . . . . . . . . . . . . . . . . . . . . . . . . .

The Crystal Structure Determination of $\mathrm{CuPt}_{3} \mathrm{O}_{6}$ from Powder Diffraction Data

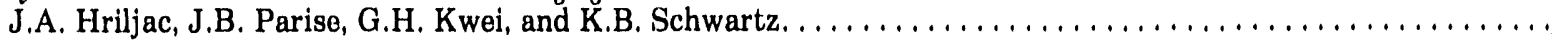

The Effects of High-Pressure Annealing on the Phase Transitions in $\mathrm{La}_{1,88} \mathrm{Ba}_{0.12} \mathrm{CuO}_{4}$

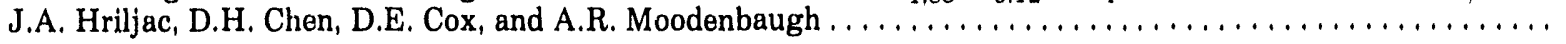

Atomic Structure of $\mathrm{Al}_{65} \mathrm{Ru}_{15} \mathrm{Cu}_{20}$

R. Hu, T. Egami, A.P. Tsai, A. Inoue, and T. Masumoto. . . . . . . . . . . . . . . . . . . .

Compression of Solid Krypton to $130 \mathrm{GPa}$

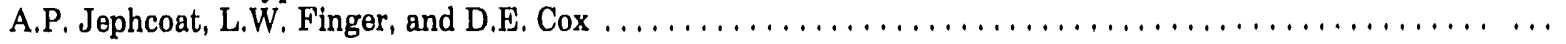

High-Resolution, High l-Pressure Powder Diffraction

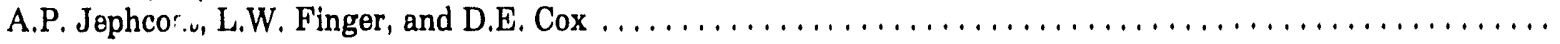

Microstructural Aspects of the LTO-LTT Transition in $\mathrm{La}_{1.875} \mathrm{Ba}_{0.125} \mathrm{CuO}_{4}$

M. Marezio, H. Takagi, B. Batlogg, P. Bordet, D.E. Cox, and Uchida . . . . . . . . . . . . . . . . .

Preliminary Structural Investigations of the (sic) Some Univalent Cation Exchanged Forms of the RHO Framework J.E. Parise, D.R. Corbin, L. Abrams, T.M. Nenoff, and G.D. Stucky . . . . . . . . . . . . . . . . . . .

Anomalous Diffraction Study of $\mathrm{Pb}\left(\mathrm{Mg}_{1 / 3}, \mathrm{Nb}_{2 / 3}\right) \mathrm{O}_{3}$

H.D. Rosenfeld and T. Egami

The Local Atomic Structure of $\mathrm{Pb}\left(\mathrm{Mg}_{1 / 3}, \mathrm{Nb}_{2 / 3}\right) \mathrm{O}_{3}$

H.D. Rosenfeld and T, Egami 
High Resolution Synchrotron X-ray Diffraction Study of Phase Separations in $\mathrm{Pr}_{2} \mathrm{NiO}_{4+\delta}$

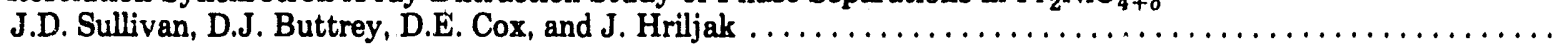

Re-Examination of the Phase Diagram in Heavily-Doped $\mathrm{La}_{2-x} \mathrm{Sr}_{\mathrm{x}} \mathrm{CuO}_{4}$ H. Takagi, M. Marezio, R.J. Cava, B. Batlogg, P. Bordet, J. Hodeau, and D.E. Cox . . . . . . . . . . . . . .

Orientational Disorder in Solvent-Free Solid $\mathrm{C}_{70}$

G.B. Vaughan, P.A. Heiney, J.E. Fischer, D.E. Luzzi, D.A. Ricketts-Foot, A.R. McGhie, Y.W. Hui,

A.L. Smith, W.J. Romanow, B.H. Allen, N. Coustel, J.P. McCauley Jr., A.B. Snith, and D.E. Cox ...........

Isothermal Compression of $\mathrm{Fe}_{2} \mathrm{SiO}_{4}$ (SPINEL) at Pressures Up to $24 \mathrm{GPa}$ at Roum Temperature

L.J. Wang, J.A. Xu, L.C. Ming, J. Balogh, and H. Manghanani. ....................... 133

High Temperature Behavior of $(\mathrm{Mg}, \mathrm{Fe}) \mathrm{SiO}_{3}$ Perovskite at $1 \mathrm{Bar}$

Y. Wang, Y. Zhao, D.J. Weidner, R.C. Liebermann, J.B. Parise, and D.E. Cox

Comparative Study of Elemental Contrast by Neutron and Resonant X-ray Powder Diffraction

J.K. Warner, A.P. Wilkinson, A.K. Cheetham, and D.E. Cox

$\mathrm{Fe}^{\mathrm{II}} / \mathrm{Fe}^{\mathrm{III}}$ Valence Contrast by Resonant X-ray Powder Diffraction

J.K. Warner, A.K. Cheetham, and D.E. Cox

Reproducibility of Site Occupancies Obtained from Resonant X-ray Powder Diffraction

J.K. Warner, A.P. Wilkinson, A.K. Cheetham, and D.E. Cox

Atomic In-Plane Disorder in Co/Pt Superlattices

X.Yan, T. Egami, E.E. Marinero, R.F.C Farrow, and C.H. Lee

Atomic Interdiffusion in $\mathrm{Co} / \mathrm{Pt}$ Superlattices

X. Yan, T. Egami, E.E. Marinero, R.F.C. Farrow, and C.H. Lee.

Perovskite Structure as Function of Temperature \& Pressure: The Rosults from $\mathrm{NaMgF}_{3}$ Perovskite

Y. Zhao, D.J. Weidner, J.B. Parise, and D.E. Cox

\section{Beamline X7B}

High Pressure Single Crystal X-ray Diffraction Investigations of $\mathrm{CaF}_{2}$ : Data Collection at $6.7 \mathrm{GPa}$ and at Norma' Pressure

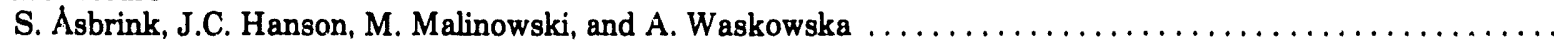

Single Crystals with Intercalated Proteins from Biomineralized Materials

A. Berman, L. Addadi, L.Leiserowiz, S. Weiner, J. Hanson, and T. Koetzle

Study of the Low Temperature Structure of the $2212 \mathrm{Tl} \cdot \mathrm{Ba} \cdot \mathrm{Ca}-\mathrm{Cu}-\mathrm{O}$ Superconductor

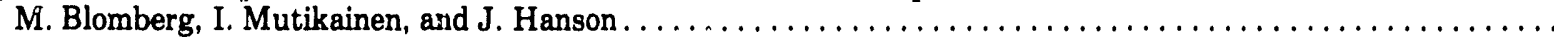

Diffraction Linewidth of Charge-Density Wave Satelite Spots

S.M. DeLand, G. Mozurkewich, and L.D. Chapman

Single Crystal Study of Hydrated Aluminophosphate at $20 \mathrm{~K}$

T. Ericksson, A. Kvick, Y. Long, and J.C. Hanson.

EXAFS Investigation of Amorphous Platinum Uridine Clusters in $\mathrm{H}_{2} \mathrm{O}$-Solution

V. Eteläniemi, R. Serimaa, T. Laitalainen, T. Paakkari, and I. Mutikainen.

\section{Beamline X9A}

EXAFS Studies of Copper-Containing Monooxygenases

N.J. Blackburn and R.S. Strange

X-ray Diffraction Studies of Tethered Protein Monolayers and Ultrathin Lipid Multilayer Films on Ge/Si Multilayer Substrates

J.K. Blasie, J. Chupa, S. Xu, M. Murphy and R. Fischetti

Time-Resolved, Resonance X-ray Diffraction Studies of Sarcoplasmic Reticulum Membrane Multilayers

J.K. Blaise, F. Asturias, L. DeLong and R. Fischette

XAFS Studies of the Iron Containing Subunit of Ribonucleotide Reductase

G. Bunker, K. Zhang, B. Chance, M. Sahlin, and B.-M. Sjöberg .

The Structure of the Transient State Intermediate of the $\mathrm{H}^{+}$-ATPASE from Chloroplasts

C. Carmeli, O. Tadmor, R. Ophir, and S. Carmeli 
In-Situ XANES Study of the Chemistry of Passive Oxides on AlV and AlMn Thin Films Under Electrochemical

Control

A.J. Davenport, H.S. Isaacs, G.S. Frankel, A.G. Schrott, C.V. Jahnes, and M.A. Russak . . . . . . . . . . . . . . 142

Purification, EXAFS, and Edge Data of Vitamin-B12 Binding Proteins with Cyano-and Aquo-Cobalamin

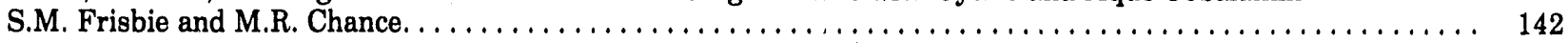

EXAFS Studies of Ni Tetraazamacrocycles and Their Adducts as Models of F430

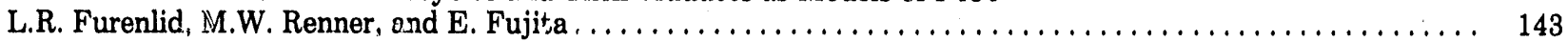

In-Situ Characterization of a Copper Monolayer Electrochemically Adsorbed on a Platinum Electrode

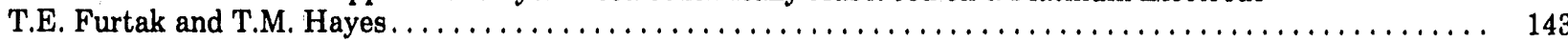

The Structure of the Zinc Sites of E. coli DNA-Dependent RNA Polymerase

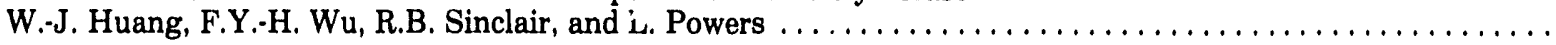

Drug Structures In Biological Membranes

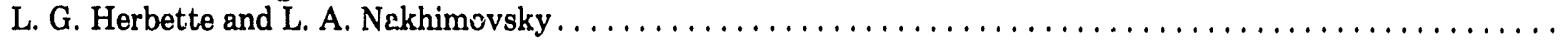

Discotic Liquid Crystal Strands

S.H.J. Idziak, N.C. Maliszewskyj, G.B.M. Vaughan, P.A. Heiney, J.P.McCauley, Jr. A.B. Smith, III,

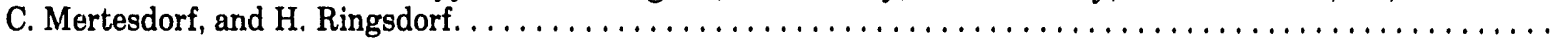

$\mathrm{X}$-Ray Absorption Spectroscopy of Manganese in Chloroplasts

M.P. Klein, V.K. Yachandra, V.J. DeRose, M.J. Latimer, I. Mukerji, and K. Sauer. . . . . . . . . . . . . . . 145

EXAFS Experiments on Iron, Copper, and Zinc Proteina

R. Korszun.

Protein Damage and Possible Protection: EXAFS Studies of Metallo Enzymes

C. Kumar, K.S. Redidy, C. Lee, and B. Chance .

$\mathrm{X}$-ray Induced Damage to Proteins During EXAFS Studies

C. Kumar, B. Chance, and K.S. Reddy .

The Vertical X-ray Beam Stability at Beam Lines X-9A \& B at NSLS

H.-C. Lee, S. Khalid, M.-Z. Zhang, and B. Chance.

Glancing-Angle XAFS Studies of Premelting at $\mathrm{Cu} / \mathrm{Al}_{2} \mathrm{O}_{3}$ Interfacial Regions

R.A. Mayanovic, B.A. Bunker, and N. Jisrawi

Polarized X-ray Absorption Spectroscopy

J.E. Penner-Hahn, G.S. Waldo, K. Clark, and T. Stemmler

Hemeprotein Catalysis: Structure $\leftrightarrow$ Function Relationship

L. Powers, R.B. Sinclair, C.-S. Chang, S. Hallam, and I. Yamazaki

EXAFS Studies of $\left[\left(\mathrm{HB}\left(3,5 \cdot{ }^{\mathrm{i}} \mathrm{Pr}_{2} \mathrm{pz}\right)_{3}\right) \mathrm{Fe}^{\mathrm{II}}(\mathrm{OBz})(\mathrm{MeCN})\right](1)$

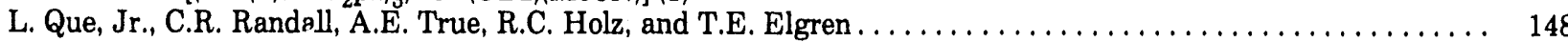

EXAFS Investigations of E7 Mutant Myoglobins

K.S. Reddy, L. Powers, H. Tang, and B. Chance.

Photomultiplier/Phosphor X-ray Detector for EXAFS Measurements

K.S. Reddy, S. Khalid, C. Kumar, and B. Chance

Prototype Stopped Flow Apparatus for EXAFS Study of Enzyme Intermediates

K.S. Reddy, C. Kumar, and B. Chance.

Improved Sagittal Focus of Scanning Monochromator

G. Rosenbaum, M. Sullivan, and L. Rock

Small Angle Diffraction Pattern from Smooth Muscle

G. Rosenbaum and A. Somlyo.

EXAFS Structure of Retroviral Gag Z̈inc Finger Protein-RNA Complex in Intact Retroviral Particles

I. Sagi, L.O. Arthur, L.E. Henderson, M.F. Summers, J.W. Bess, and M.R. Chance . . . . . . . . . . . . . . .

EXAFS Studies of Zinc Finger Binding Proteins

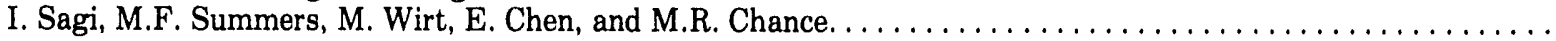

SIT Detector Characterization

V. Skita, R. Kelly

Ligninase Active Site Structure

R. Sinclair, I. Yamazaki, J. Bumpus, B. Brock, C.-S. Chang, A. Albo, and L. Powers . . . . . . . . . . . . . . . . 
EXAFS of $\mathrm{Fe}^{3+}$ in Active Soybean Lipoxygenase

Solution Scattering Studies of the Polyphenolic Protein Isolated from the Blue Mussel Mytilus Edulis M.W. Trumbore, V. Skita, and R. Kelley .

XAS Investigations Into the Structural Conformations of the Redox States of Hydrogenase from Thiocapsa

Roseopersicina

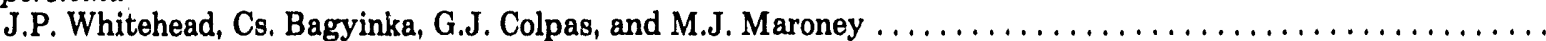

Ge Detector Dead Time Loss and Its Effect on XAFS

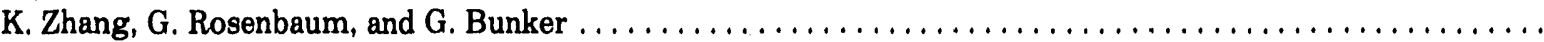

XAFS Studies of Carboxypepptidase and its Intermediate State

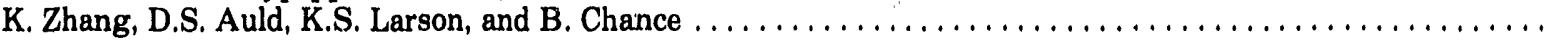

An X-ray Absorption Study on Pro-carboxypeptidase

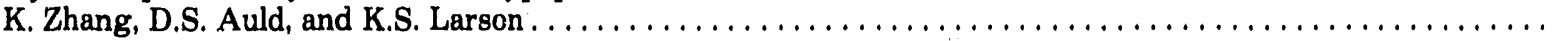

An XAFS Study on HPV-16 E7 Protein

K. Zhang and D. Patrick

\section{Beamline X10A,B}

Transverse Structure Function of the Liquid-Vapor Interface of $\mathrm{Ga}$

A. Acero, Z.H. Cai, E. Flom, B.-H. Lin, N. Maskil and S.A. Rice

Direct Phase Determination of Large Macromolecule Crystals Using Three-Beam X-Ray Interierence

S.-L. Chang, H. E. King, Jr., M.-T. Huang, and Y. Gao .

High Resloution Small Angle X-ray Scattering Study of Styrene-Butadiene Diblock Copolymer

S.-L. Chang, K.S. Liang, Z. Fu, R.J. Rose, and K.J. Joen.

Phase Determination for Macromolecular Crystals Using X-ray Multiple Diffraction

S.-L. Chang, H. King, M.T. Huang, and Y. Gao

GIXD Study of Self-Assembled Monolayers

P. Fenter, J. Li, P. Eisenberger, T.A. Ramanarayanan, and K.S. Liang

Misfit Dislocations in the Interface of Epitaxial Nb Films un Sapphire

C.H. Lee, and K.S. Liang .

The Structure of Dilute Lamellar $\mathrm{L}_{\alpha}$ Phases of Surfactant Membranes

N. Lei and C.R. Safinya

Surface-Induced Order on a $\mathrm{Cu}_{3} \mathrm{Au}(001)$ Surface

K.S. Liang, H.H. Hung, Z. Fu, and S.-L. Chang

High Resolution Powder Diffraction Studies of Disorder in Lithium Nickel Oxides

I.J. Pickering, J.T. Lewandowski, and A.J. Jacobson .

Location of Iron in Framework-Substituted Zeolite L Using High Resolution Powder Diffraction

I.J. Pickering, D.E.W. Vaughan, and K.G. Strohmaier

Effect of Excess Gold on the Surface Ordering Kinetics of the $\mathrm{Cu}_{3} \mathrm{Au}(001)$ Surface

S.B. Rivers, W.N. Unertl, H.H. Hung, K.L. D'Amico, and K.S. Liang.

Melting and Reassembling Transition of the 2-D Protein Latiice of Bacteriorhodopsin

Y. Shen, C.R. Safinya, K.J. Rothschild, and B. Thomas

X-ray Diffraction Studies of Phospholipid-Based-Tubules

B. Thomas, C.R. Safinya, B. Ratna, R. Shashicunar, and N.A. Ciark.

Specular and Off-Specular X-ray Reflectivity from Sputtered $\mathrm{Fe} / \mathrm{Cr}$ Multilayers

M.F. Toney and S.S.P. Parkin.

Observation of Ordered $\mathrm{CoPt}_{3}$ in Single Crystal Magnetic Multilayers

M.F. Toney, R. Farrow, and R. Marks .

X-ray Reflectivity Study of Oxidation Induced Roughening of the Pt(111) Single Crystal Surface Under In-Situ

Electrochemical Potential Control

H. You, D.J. Zurawski, Z. Nagy, R.P. Chiarello, H.K. Kim, and R.M. Yonco . . . . . . . . . . . . . . . . .

Pressure-Induced Staging Transition in $\mathrm{TiS}_{2}$ Intercalcation Compounds

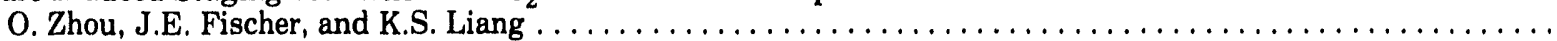


Structure of $M_{6} C_{60}(M=K, R b$, and $C s)$

Vol II

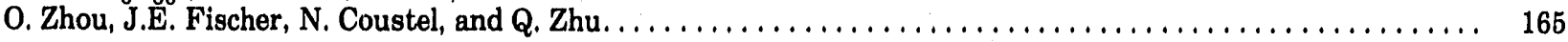

$\mathrm{X}$-Ray Diffraction Study of $\mathrm{Rb}_{x} \mathrm{C}_{60}$ Phase Diagram

Q. Zhu, O. Zhou, N. Coustel, G. Vauhan, and J.E. Fischer $\ldots \ldots \ldots \ldots \ldots \ldots \ldots \ldots \ldots \ldots \ldots \ldots \ldots \ldots \ldots$

\section{Beamline X10C}

XANES of Molybdenum Compounds and Catalysts at the Molybdenum $\mathrm{L}_{2,3}$.Edges

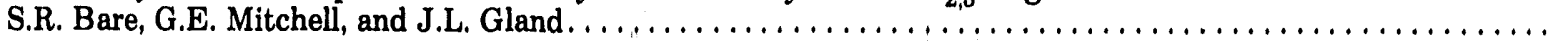

XAFS of Two Novel Tungsten-Containing Enzymes from the Hyperthermophilic Bacteria Pyrococcus Furiosus and Thermococcus Litoralis

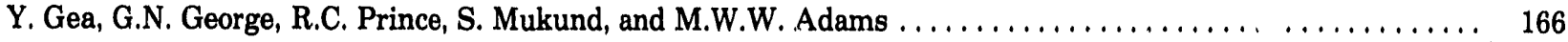

Polarized Single Crystal Sulfur K-Edge X-ray Absorption Spectroscopy of Substituted Thiophenes

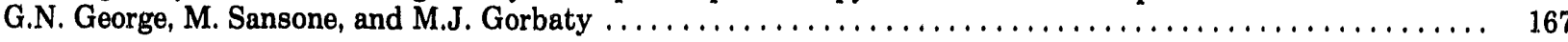

X-ray Absorption Spectroscopy of Zinc Dialkyl Dithiophosphates

G.N. George, M. Sansone, and D. Martella .................................... 167

X-ray Fluorescence XAFS Microscopy at $50 \mu \mathrm{m}$ Resolution

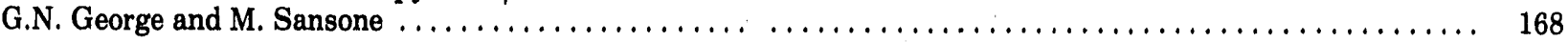

Polarized EXAFS Studies of Nitrogenase Crystals

S.L. George, J. Chen, J. Christiansen, S.P. Cramer, N. Campobasso, J. Bolin, M. Sansone, and

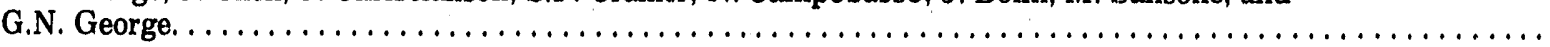

XAS Study of Metal Partitioning at Water/Oxide Interfaces

K.F. Hayes, L.E. Katz, and J.E. Penner-Hahn

In-Situ XANES of an Iron Porphyrin Irreversibly Adsorbed on an Electrode Surf?ce

S. Kim, I.T. Bae, M. Sandifer, P.N. Ross, R. Carr, J. Woicik, M.R. Antonis, and D. Scherson. . . . . . . . . . .

Investigation of the Chemistry of Chromium in Anti-Corrosion Finishes on Aluminum

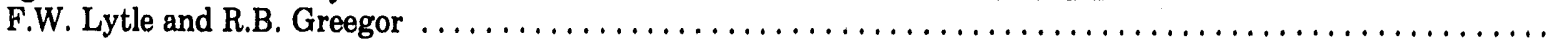

Near-Edge vs EXAFS Studies of Oxides: Understanding Without the Tears

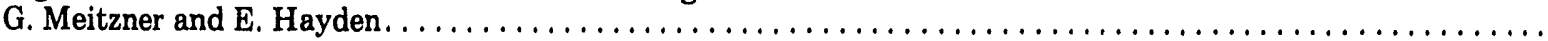

DIFFRAXAFS: A Novel Technique for Probing Local Atomic Environment

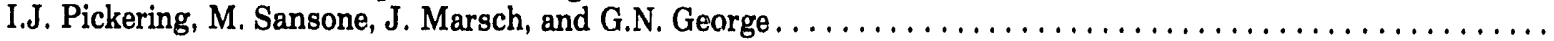

X-ray Absorption Spectroscopy of Transition Metal Substituted Zeolites

I.J. Pickering, G.N. George, G.H. Via, D. Sondericker, K.G. Strohmaier, and D.E. Vaughan. . . . . . . . . . .

Sulfur K-Edge X-ray Absorption Studies of Photographic Materials

T. Smith, J. DeWitt, K. Hodgson, and B. Hedman. . . . . . . . . . . . . . . . . . . . . . . .

\section{Beamline X11A}

Titanium Germanide Formation

D.B. Aldrich, C.L. Jahncke, R.J. Nemanich, and D.E. Sayers

Annular Detector for Obtaining Accurate X-ray Absorption Spectra in Fluorescence from Thick Concentrated

Samples

D.L. Brewe, D.M. Pease, P. Chartier, J.I. Budnick, C.E. Bouldin, and Z. Tan

Morphology of Supported Platinum Catalysts Prepared from Platinum Carbonyls on Magnesium Oxide

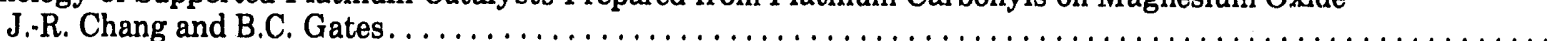

Genesis of Pt-Re Bimetallic Interactions in Supported Pt-Re Catalysts

J.-R. Chang, S.K. Purnell, and B.C. Gates.

Electron-Yield and Fluorescence XAFS Study of E-Beam Deposited Co/Pd Multilayers M. Choi, J.H. Joo, S.K. Kim, J.S. Kang, Y.-P. Lee, S.C. Shin, and S.M. Heald

Electron-Yield EXAFS Study of Multilayers for Magneto-Optical Device Materials M. Choi, J.H. Joo, J.G. Lee, C.S. Yang, K. Kim, D. Whang, and S.M. Heald

XAFS of Strained Discontinuous Au Films

G.A. DeRose and R.W. Hoffman 
XA i'S of Chemically Synthesized II.VI Nanoclusters

Glarw ing Angle X-ray Study of Crystallization of Amorphous Ge at the Ge-Al Interface

S.M. Heald and J.K.D. Jayanetti .

Operation of a Dynamically-Bent Sagittally-Focussing Double Crystal Monochromator for XAFS Studies

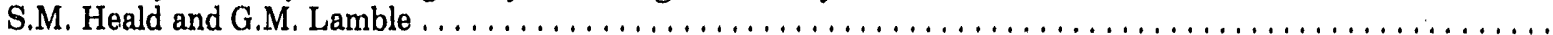

Interfacial Reactions of Ni-Cr Alloys and Aluminum

S.M. Heald, Z. Tan, and J. Jayanetti .

X-ray Reflectivity Study of Thin Film Density for $\mathrm{Al}, \mathrm{Cu}, \mathrm{Ni}, \mathrm{Co}, \mathrm{Cr}, \mathrm{Mn}$, and Pd

S.M. Heald, Z. Tan, and J.K.D. Jayanetti

Ligand Effects in Supported Metal Carbonyls: X-ray Absorption Spectroscopy of Rhenium Subcarbonyls on Magnesium Oxide

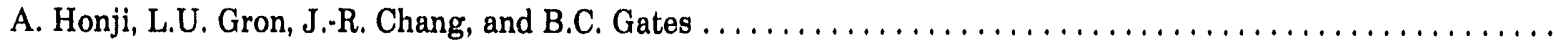

EXAFS Studies of the Interfacial Phase of Highly Milled Sn/Ge Composites

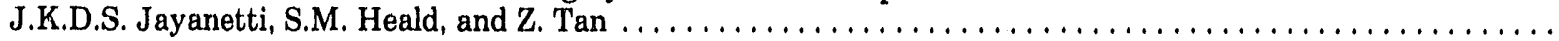

$\sigma^{2}$-Derivative Method for Disorder in $\mathrm{PbTiO}_{3}$

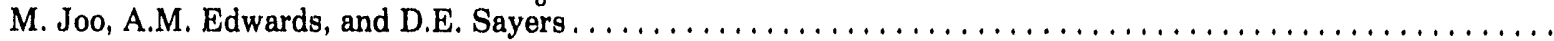

Slope Analysis of Disorder in $\mathrm{SrTiO}_{3}$

M. Joo, A.M. Edwards, and D.E. Sayers

Temperature-Dependent EXAFS Study of $\mathrm{Sr}_{0.8} \mathrm{~Pb}_{0.2} \mathrm{TiO}_{3}$

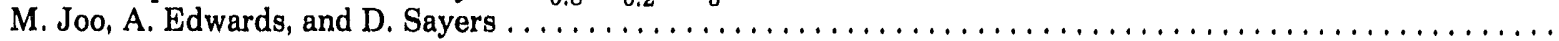

Measurement of Internal Strains Using Embedded Micromarkers

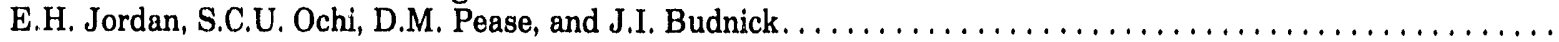

XANES of Conversion Coatings on Aluminum Alloys

M. Kendig, A. Davenport, and H. Isaacs. . . . . . . . . . . . . . . . . . . . . . . . .

A Time-Resolved Diffraction Study of the Ta-C Combustion System

E.M. Larson, J.B. Holt, J. Wong, P.A. Waide, B. Rupp, and L. Terminello . . . . . . . . . . . . . . . . . . .

Comparison Between Therium and Cerium Doping and Study of the Role of Reduction for $\eta$-Type Superconductor

Related Systems

G. Liang, Y. Guo, D. Badresingh, M. Croft, J. Chen, J. Peng, R.F. Jardim, E.A. Early, M.B. Maple,

O. Beom-hoan, J.T. Markert, M. Nagoshi, and T. Suzuki.

Electronic Structure Study of $\mathrm{Nd}_{2-\mathrm{x}} \mathrm{Ce}_{\mathrm{x}} \mathrm{CuO}_{4}$ by Polarization X-ray Absorption Near-Edge Structure

G. Liang, W. Xu, M. Croft, J. Chen, J. Peng, O. Beom-hoan, and J.T. Markert .

$\mathrm{X}$-ray Absorption Study of Electron-Doped High-T $\mathrm{T}_{\mathrm{c}}$ Superconductor-Related Compounds $\mathrm{R}_{2-\mathrm{x}} \mathrm{Ce}_{\mathrm{x}} \mathrm{CuO}_{4-\delta}$

G. Liang, Y. Guo, D. Badresingh, W. Xu, M. Croft, J. Chen, J. Peng, O. Beom-hoan, and J.T. Markert . . . . . . .

Thermal Vibrations and Melting from a Local Perspective

P. Livins, E.A. Stern, and 7. Zhang

XANES and EXAFS Study of Mn Doped $\mathrm{CaF}_{2}$ Dosimetric Phosphor

A.N. Mansour and J.B. Barkyoumb

Characterization of a $\mathrm{Cu} / \mathrm{Al}_{2} \mathrm{O}_{3}$ Buried Interface Using Glancing-Angle X-ray Reflectivity and XAFS Studies

R.A. Mayanovic, B.A. Bunker, C. Zhong, and A. Anagnostopoulos . . . . . . . . . . . . . . . . . .

Glancing-Angle XAFS Studies of Premelting at $\mathrm{Cu} / \mathrm{Al}_{2} \mathrm{O}_{3}$ Interfacial Regions

R.A. Mayanovic, B.A. Bunker, and N. Jisrawi $\ldots \ldots \ldots \ldots \ldots \ldots \ldots \ldots \ldots \ldots \ldots \ldots \ldots \ldots$

EXAFS Investigations of $\mathrm{Pt}-\mathrm{Au} / \gamma \cdot \mathrm{Al}_{2} \mathrm{O}_{3}$ Catalysts

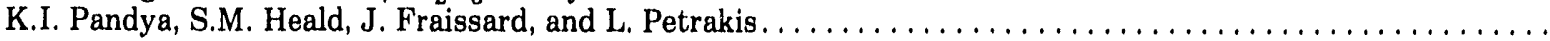

$\mathrm{L}_{3,2}$ Core Hole Lifetime Widths of $3 \mathrm{~d}$ Transition Metals

D.M. Pease

The Structural Characterization of Metal Oxide Clusters in Zeolite-Y and Zeolite-L Hosts, Produced by the

Photo-Oxidation of Group(VI) Metal Hexacarbonyls, Followed by Various Degrees of Subsequent Reduction

R.A. Prokopowicz, A. Malek, M. Steele, A. Stein, and G.A. Ozin

Spontaneous Ordering EXAFS Study of $\mathrm{Ga}_{0.5} \mathrm{In}_{0.5} \mathrm{P}$ Thin Films Deposited on $\mathrm{GaAs}$

A.Tabor-Morris, K. Kemner, R. Mayanovic, and B. Bunker 
Rare Earth Valence and Doping in T-, T'-and T*-Phase $\mathrm{R}_{2} \mathrm{CuO}_{4}(\mathrm{R}=\mathrm{Rare}$ Earths)

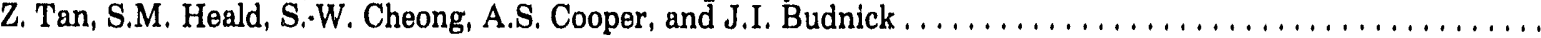

$\mathrm{X}$-ray-Absorption Studies of Local Structure of $\mathrm{LaRSrCu}_{2} \mathrm{O}_{6+y}(\mathrm{R}=\mathrm{La}, \mathrm{Pr}, \mathrm{Nd}, \mathrm{Sm}$ and $\mathrm{Gd})$

Z. Tan, S.M. Heald, S.-W. Cheong, H. Hwang, A.S. Cooper, and L.W. Rupp, Jr. . . . . . . . . . . . . . . . . . . . . 188

EXAFS Study of $\mathrm{Fe}$ Core Structure in Reconstituted Ferritins

Q. Wang, A. Edwards, D. Sayers, and E. Theil

EXAFS Study of Local Structure of Ferroelectric $\mathrm{Zn}_{\mathrm{x}} \mathrm{Cd}_{1-\mathrm{x}} \mathrm{Te}$ Alloys

Z. Wang, R. Mayanovic, B. Bunker, U. Debska, and J. Furdyna

EXAFS and X-ray Edge Studies of Cobalt Substituted Bacteriorhodopsin: Irreversible Light Induced Cation Uptake M.D. Wirt, M.R. Chance, and M. Engelhard

$\mathrm{X}$-ray Absorption Near-Edge Study of Several Bi- and Tl-Based High- $\mathrm{T}_{\mathrm{c}}$ Superconductor Systems

W. Xu, G. Liang, M. Croft, J. Chen, J. Peng, R.S. Liu, B. Jayaram, and J.M. Tarascon . . . . . . . . . . . . . . . . .

Trends in Protein Structure Changes Around the Transition Metal Site in Concanavalin A When the Crystallized

Protein is Dissolved

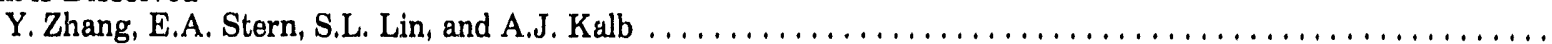

Structure and Photo-Induced Structural Changes in Non-Stoichiometric a- $\mathrm{As}_{\mathrm{x}} \mathrm{S}_{1-\mathrm{x}}$

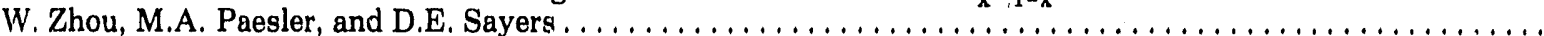

\section{Beamline X12B}

X-ray Diffraction Studies of Liquid Crystalline Polymers

P. Cebe, Y. Aihara, P. Huo, C. You, H. Gibson, A. Bluhm, and W. Yeomans . . . . . . . . . . . . . . . . . .

SAXS Study of the Folding of a Globular Protein in the Presence and Absence of Chaperonins

J. Flanagan, M. Kataoka, M. Capel, and D. Engelman

The Role of Histone $\mathrm{H} 1$ in Chromatin Higher-Order Structure

V. Graziano, M. Chapel, and V. Ramakrishnan

X-ray Diffraction Studies of Blends of Poly(Butylene Terephthalate) with Polyarylate

P. Huo, C. You, Y. Aihara, and P. Cebe

$\mathrm{X}$-ray Diffraction from Mixtures of Collagen Types I and III

V. James, J. McConnell, and M. Capel

Phase Study of AOT/Water/Decane Dense Microemulsion

E.Y. Sheu, M.M. De Tar, and M. Capel

Properties of Vacuum Residue Fractions in Organic Sclventis

E.Y. Sheu, M.M. De Tar, and D.A. Storm.

SAXS Studies of Porous Carbons

J. Wergrzyn, A.N. Goland, M. Capel, and J. Schwartz. .

\section{Beamline X12C}

Structure Determination and Refinement of Bovine Lens Leucine Aminopeptidase and Its Complex with Bestatin

S.K. Burley, P.R. David, R.M. Sweet, A. Taylor, and W.N. Lipscomb. . . . . . . . . . . . . . . . . .

Three-Dimensional Structure of T7 Lysozyme

X. Cheng, J.W. Pflugrath, X. Zhang, and F.W. Studier.

Structural Studies of Tryptophanyl-tRNA Synthetase Using Multiwavelength Anomalous Dispersion from

Selenomethionine-Substituted Protein Crystals

S. Doublie, R.M. Sweet, and C.W. Carter, Jr.

Structural Studies of the Malic Enzyme from the Parasitic Nematode ASCARIS SUUM and the Interleukin-1

Receptor Antagonist Protein

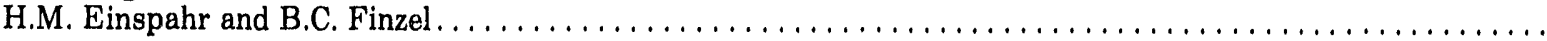

Probable Amylase/Protease Inhibitor-B from Rice Seeds

K.Y. Hwang, K.K. Kim, and S.W. Suh. .

Thermostable $\alpha$-Amylase from B. Licheniformis

K.Y. Hwang, K.K. Kin, and S.W. Suh. 
X-ray Crystallography of Arylesterase

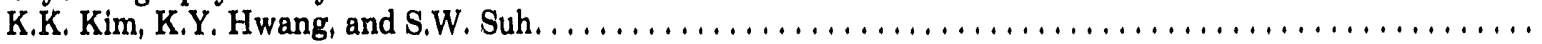

X-ray Crystallography of Lipase

K.K. Kim, K.Y. Hwang, and S.W. Suh.

Structural Studies on Flavo-Protein Reductables

J. Kuriyan, T.S.R. Krishna, B. Guenther, X.P. Kong, and R.M. Sweet . . . . . . . . . . . . . . . . . . .

The Structures of DNA and GM-CSF

J. LaLonde, K. Swaminathan, R.M. Sweet, and D. Voet

Structural Analysis of the E. Coli TRP Repressor L-Tryptophan Binding Pocket: Why 5-Methyltryptophan is a

Better Corepressor

C.L. Lawson and R.M. Sweet

Structural Basis of Latency in Plasminogen Activator Inhibitor-1

J. Mottenen, A. Strand, J. Symersky, R.M. Sweet, D.E. Danley, K.F. Geoghegan, D. Gerard,

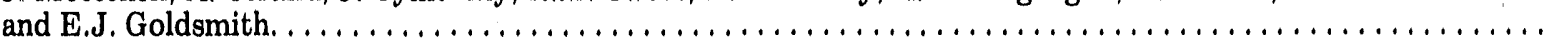

Three-Dimensional Structure of cAMP-Dependent Protein Kinase

J.W. Pflug: ath, J. Kuret, X. Cheng, and R.M. Sweet

Crystal Structure Determination of Ribosomal Protein S5

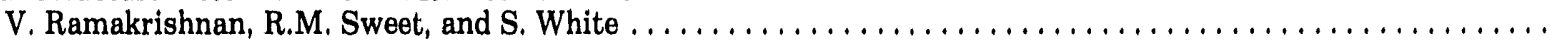

Structure of the Globular Domain of Histone H5

V. Ramakrishnan, V. Graziano, and R.M. Sweet.

Crystallographic Studies of Complexes of Bovine Somatotropin with a Soluble Fragment of its Receptor, Renin and

EPSP Synthase

H-S. Shieh, R.A. Stegeman, A.M. Stevens, W.C. Stallings, and R.M. Sweet. . . . . . . . . . . . . . . . .

A Comparison of High-Resolution Tryspin Crystal Structures Obtained by White-Beam Laue and Monochromatic

$X$-ray Diffraction Using Synchrotron Radiation

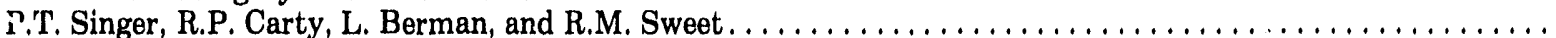

Multiwavelength Data Collection on BamHI

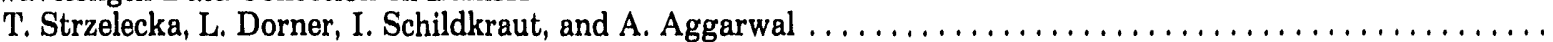

Structure Determination of Staphylococcal Enterotoxin $B$

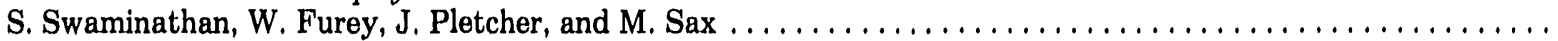

\section{Beamline X14}

Diffuse X-ray Scattering from the Alloy Fe-25\% Ni-1\%C

B.D. Butler, P.R. Zschack, H. Yalcinkaya, and J.B. C(ihen

Studies of the Structure of Amorphous Melanins

J. Cheng, M. Eisner, and S.C. Moss

Isothermal Thickening and Thinning Processes in Low MW PEO Fractions Observed From Synchrotron SAXS

S.Z.D. Cheng, A.-Q. Zhang, J.S. Barley, J.-H. Chen, A. Hubenschuss, and P.R. Zschack . . . . . . . . . . . . . .

Powder Diffraction Study of Magnetoelastic Strain Broadening in Nanocrystalline Dysprosium

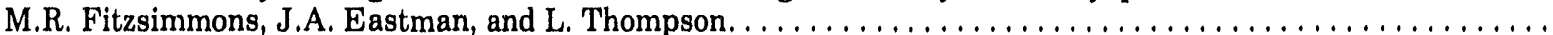

X-ray Scattering Study of Ag/Si(111) Buried Interface Structures

H. Hong, R.D. Arburano, D.-S. Lin, H. Chen, T.-C. Chiang, P. Zschack, and E.D. Sł ıecht. . . . . . . . . . . . . .

Inelastic X-ray Scattering Spectroscopy in Lithium Hydride

E.D. Isaacs, G.E. Ice, and P. Zschack

Crystallographic Study of a Single Quasicrystal of Al-Cu-Fe

X.B. Kan, S.C. Moss, J.L. Robertson, T. Ishimasa, M. Mori, D. Gratias, V. Elser, and P. Zschack

$\mathrm{X}$-ray Determination of Site Occupation Parameters in Ordered Ternaries $\mathrm{Cu}\left(\mathrm{Au}_{\mathbf{x}} \mathrm{M}_{1-\mathrm{x}}\right) \mathrm{M}=\mathrm{Ni}, \mathrm{Pd}$ : Anomalous

Scattering and Rietveld Refinement

R. Kumar, C.J Sparks, E.D. Specht, P. Zschack, G.E. Ice, T. Shiraishi, and K. Hisatsune . . . . . . . . . . . . .

Direct Determination of Precip itate Strain and Sizes Using High-Order X-ray Diffuse Scattering

B.C. Larson, M.D. Galloway, G.E. Ice, and P. Zschack 
Magnetic X-ray Scattering in Dy/Lu Superlattices

M.B. Salamon, R.S. Beach, B.A. Everitt, K. Pettit, A. Matheny, and C.P. Flynn . . . . . . . . . . . . . . . . 210

X-ray Studies of Dysprosium/Lutetium Superlattices

M.B. Salamon, R.S. Beach, C.P. Flyn n, A. Metheny, and J. Borchers

Anomalous Behavior of Diffraction Peak Withs in Lipid Monolayers

M.C. Shih, T.M. Bohanon, J.M. Mikrut, P. Dutta, and P. Zschack.

Effect of $\mathrm{pH}$ on a Fatty Acid Monolayer with Calcium Ions in the Subphase

M.C. Shih, T.M. Bohanon, J.M. Mikrut, P. Dutta, and P. Zschack.

High-Pressure Phases of a Lipid Monolayer and Their Relationship to Isotherm Anomalies

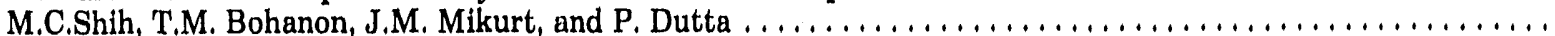

Time-Domain Spectrum of Resonantly Filtered X-rays from a Nuclear Bragg Monochromator

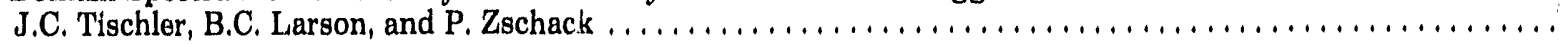

Thermal Effects on Solid Neon

C.T. Venkataraman, R.O. Simmons, M.A. Fradkin, and I. Fujita . . . . . . . . . . . . . . . . . . . .

Study of Alkoxide-Derived Amorphous $\mathrm{THO}_{2} \mathrm{Po}$ wder

Q.J. Wang, S.C. Moss, M. Shalz, A.M. Glueser, H.W. Zandbergen, and P. Zschack.

\section{Beamline X15A}

Structural Study of Si-Alkali Metal Interface by X:SW

S. Lagomarsino, F. Scarinci, P. Castrucci, C. Giannini, J.R. Patel, and E. Fontes . . . . . . . . . . . . . . . . . .

X-ray Standing Waves: $\mathrm{YBa}_{2} \mathrm{Cu}_{3} \mathrm{O}_{7}$ on $\left.\mathrm{SrTiO}_{3}(100)\right)$ and $\mathrm{Si}(111)$

J. Zegenhagen, H.-U. Habermeir, T. Siegrist, J.R. Patel, and E. Fontes . . . . . . . . . . . . . . . . . . . . .

\section{Beamline X16A}

In-Situ X-ray Diffraction Study of Silicide Growth

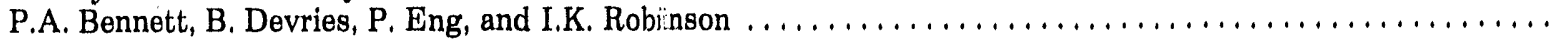

Monolayers of $\mathrm{Pb}$ on $\mathrm{Si}(111)$

K. Evans-Lutterodt, E. Fontes, B.J. Hinch. L.H. Dubois, and J. Patel . . . . . . . . . . . . . . . . . . . .

Adsorption Geometry of Antimony on Si(001) $2 \times 1$

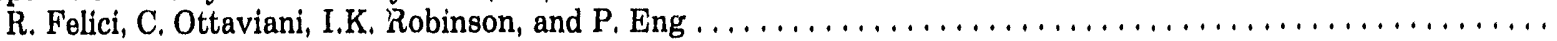

The Structure of $\mathrm{K}$ Monolayers on $\mathrm{Cu}(100)$

H.L. Meyerheim, V. Jahns, W. Mortiz, H. Schulz, I.K. Robinson, and P.J. Eng. . . . . . . . . . . . . . . . 216

Oxygen and Sulfur Structures on th: $\mathrm{Cu}(111)$ Surface

I.K. Robinson, R. Feidenhans'l, F. Besenbacher, and F. Jensen . . . . . . . . . . . . . . . . . . .

X-ray Diffraction Under Grazing Incidence on $\mathrm{Si}^{+}$Implanted Silicon After Thermal Annealing

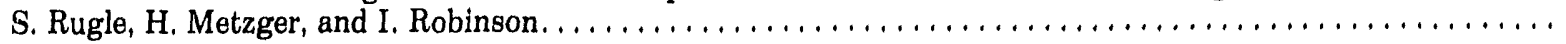

\section{Beamline X16B}

Investigation of MBE-Grown Iron Silicide Films

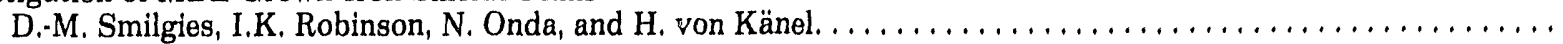

Hydrocarbon Films on Silicon I: The Structure of Wetting Films

I.M. Tidswell, T.A. Rabedeau, S.D. Kosowsky, and P.S. Pershan.

Hydrocarbon Films on Silicon II: The Structure of Chemisorbed $\mathrm{C}_{18}$ Alkylsiloxane Monolayers

I.M. Tidswell, T.A. Rabedeau, S.D. Kosowsky, P.S. Pershan, J.P. Folkers, and G.M. Whitesides ...........

\section{Beamline X17B1}

DPA Phantom Studies with a Preliminary MECT System

F.A. Dilmanian, E. Nachaliel, R.F. Garrett, W.C. Thomlinson, L.D. Chapman, N.F, Gmür, N. Lazarz,

H.R. Moulin, T. Oversluizen, H. Rarback, M. Rivers, D.N. Slatkin, P. Spanne, P.M. Stefan, A.C. Thompson,

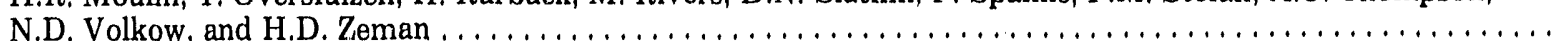


Biological Efficacy of the Auger Effect

B.H. Laster, W.C. Thomlinson, C. Gordon, N, Gmür, N, Lazarz, V. Benary, L, Warkentein, and

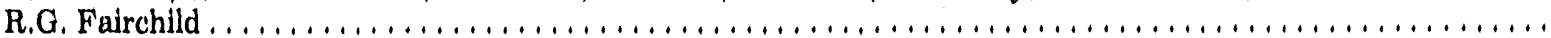

In-Situ X-ray Diffraction Study on Modified Spinel $(\beta)$ and Spinel Phases $(\gamma)$ of $\mathrm{Mg}_{2} \mathrm{SiO}_{4}$ at High Pressure and High

Temperature

Y. Meng, D.J. Weldner, G.D. Gwanmesia, R.C. Liebermann, M.T, Vaughan, R.E. Pacalo,

A. Yeganeh-Haeri, K, Leinenweber, Y. Wang, and $Y$. Zhao . . . . . . . . . . . . . . . . . . . . . . .

Performance of the MECT/Materials Science Monochromator in the Laue-Laue Diffraction Mode

E. Nachaliel, F.A. Dilmanian, R.F. Garrett, T, Oversluizen, L.E. Berman, C. Brite, L.D. Chapman,

J.B. Hastings, N. Lazarz, A. Lenhard, H. Rarback, M. Shleifer, D.P. Siddons, P.M. Stefan, and

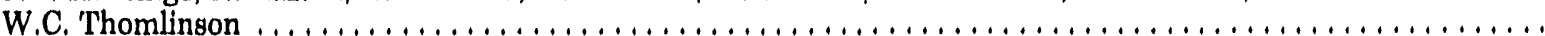

Preliminary Studies of the Potential for Structure Refinement at High Pressures Using the DIA Apparatus: $\mathrm{SiO}_{2}$ Stishovite, at High Pressure

J.B. Parise, K. Leininweber, D.J. Weidner, J, Rakovan, M. Vaughan, G. Gwanmesia,

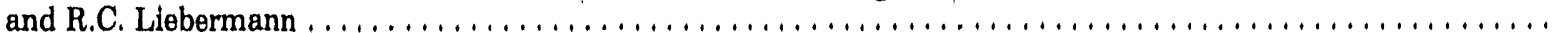

Computed Microtomography of Meteorites using a Linear Array Detector

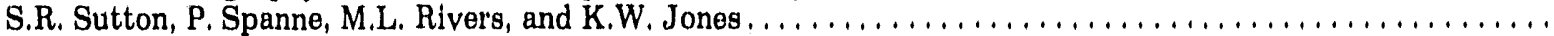

More on the High-Pressure, High-Temperature Equation of State of $\mathrm{MgSiO}_{3}$ Porovskite

D.J. Weidner, Y. Wang, R.C. Liebermann, M.T. Vaughan, K. Leinenweber, Y. Meng, R.E.G, Pacalo,

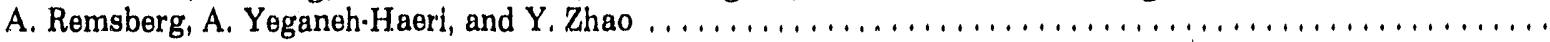

Perovskite Structure as Function of Temperature \& Pressure: - The Results from $\mathrm{NaMgF}_{3}$ Perovskite

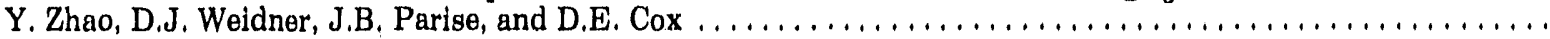

The Pressure Dependence of Transition Temperature for Structural Phase Transition in NaMgF' ${ }_{3}$ Perovskite Y. Zhao, D.J. Weidner, R.C. Liebermann, X. Liu, J. Ko, Y. Meng, R.E.G. Pacalo, J.B, Parise,

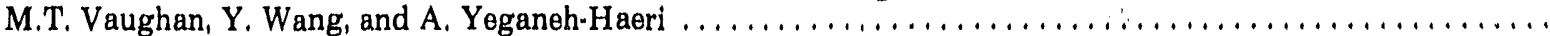

\section{Beamline X17B2}

Monochromator Harmonic Content Measurements and Calculations at Energies Above $20 \mathrm{keV}$

D. Chapman, H. Moulin, and R. Garrett .

First Human Transvenous Coronary Anglography Images at the NSLS

W. Thomlinson, N. Gmür, D. Chapman, N. Lazarz, R. Garrett, J. Morrison, P. Reiser, V. Padmanabhan,

L. Ong, S. Green, A. Thompson, H. Zeman, G. Brown, R. Hofstadter, E. Ruberistein, J. Giacomini, and

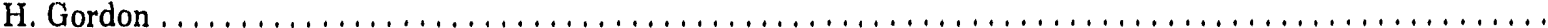

Imaging an Occluded Artery Using Transvenous Coronary Angiography

W. Thomlinson, N. Gmür, D. Chapman, N. Lazarz, R. Garrett, J, Morrison, P. Reiser, V. Padmanabhan,

L. Ong, S. Green, A. Thompson, H. Zeman, G. Brown, R. Hofstadter, E. Rubenstein, J. Giacomini, and

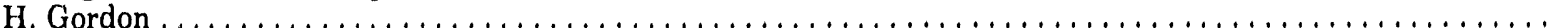

A Kinestatic Charge Detector for Intravenous Coronary Anglography Using Synchrotron Radiation X-rays

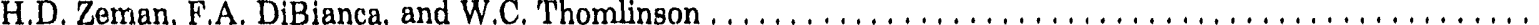

\section{Beamline X17C}

Diffraction From Single Crystals of Sub-Attoliter Volume and a New Phase of Bismuth

E.F. Skelton, L.W. Finger, S.B, Qadri, J.Z, Hu, J.D. Ayers, and H.K. Mao . . . . . . . . . . . . . . . . . .

A Method For Probing Variations in $\delta$ in $\mathrm{YBa}_{2} \mathrm{Cu}_{3} \mathrm{O}_{7-\delta}$ Single Crystals Using Energy Dispersive Diffraction

E.F. Skelton, S.B. Qadri, J.Z. Hu, M.S. Osofsky, J.L. Cohn, and T.A. Vanderah .

\section{Beamline X18A}

Early Stages of Precipitation of $\delta^{\prime}$ in $\mathrm{Al} \cdot 3.2 \mathrm{wt} \% \mathrm{Li}$

V. Mahadev, K. Mahalingam, and G.L. Liedl

A Study of Substrate-Induced Alignment of Smectic LC Phases: Presence of Surface of Substrate Stabilized Phases Near the LC-Air Interface

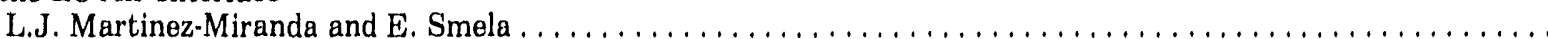

Investigation of the Local Atomic Structure in $\mathrm{HgCdTe}$

D.Q. Na, J.B. Cohen, and P. Georgopoulos 
X-ray Characterization of 10 nm Sn Film on Si(111)

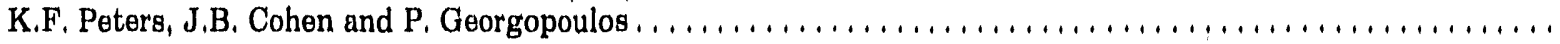

X-ray Study of Substrate-Induced Alignment on a Smectic-A Liquild Crystal

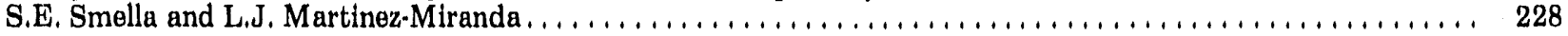

Multilayer Structure and Growth of Xenon on Ag(111)

S.-K. Wang, P. Dai, S.N. Ehrlich, T. Angot, J.Z. Larese, and H. Taub. . . . . . . . . . . . . . . . . . . . .

In-Situ X-ray Diffraction Study of YBCO Thin Films

J.Q. Zheng, S. Williams, X.K. Wang, T. Rippert, S, Maglic, D. Segel, R.P.H. Chang, J.B. Ketterson,

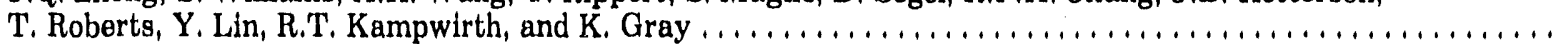

\section{Beamline X18B}

X-ray Reflectivity Study of Si/C Multilayers

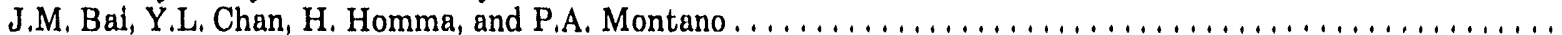

X-ray Reflectivity Study of Tl/C and W/C Multllayers

Y.L. Chan, J.M. Bai, P.A. Montano, E. Zlegler, and I.K. Schuller

Characterization by EXAFS Spectroscopy of Silver, Sodium Halo-Sodalites and Silver Oxalato-Sodalites

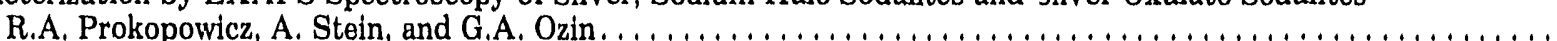

Experimental Characterization of Circular Fresnel Zone Plates

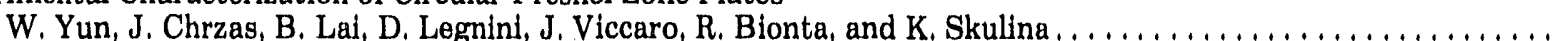

\section{Beamline X19A}

In-Situ Investigation of Passive Films on Fe-Cr Alloys

J.A. Bardwell, A.J. Davenport, H.S. Isaacs, G.I. Sproule, B. MacDourall and M.J. Graham.

Fe K-Edge EXAFS Studies of Nitrogenase

J. Chen, S.J. George, J. Christiansen, S.P. Cramer, R. Tittsworth and B. Hales . . . . . . . . . . . . . . . . . . .

$\mathrm{X}$-ray Absorption Studies of $4 d$ Transition Metal Elements

J. Chen, Y. Jeon, F. Lu, and M. Croft.

In-Situ XANES Study of Cr Valency Changes in Passive Oxides on AlCr Thin Films Under Electrochemical Control A.J. Davenport, H.S. Isaacs, G.S. Frankel, A.G. Schrott, C.V. Jahnes, and M.A. Russak . . . . . . . . . . . . . . .

Microbial Transformations of Uranium: Speciation Studies

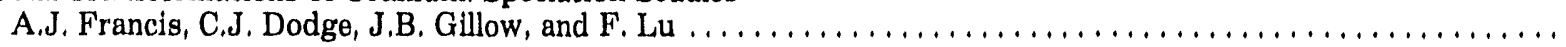

X.ray Absorption Measurements on $\mathrm{RT}_{2} \mathrm{Si}_{2}$ Compounds

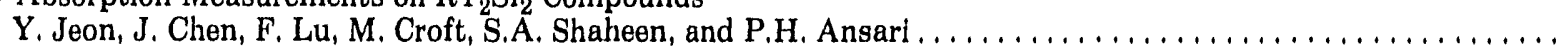

Speciation of Uranium Compounds by XANES

F. Lu, C.J. Dodge, and A.J. Francis. .

Multi-Shell Fitting of EXAFS Data in Complex Vanadium Compounds

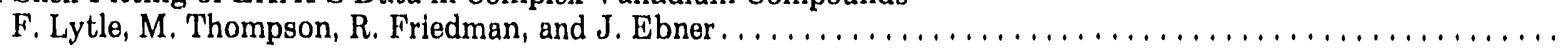

EXAFS of $\mathrm{Fe}^{3+}$ in Nitrile Hydratase

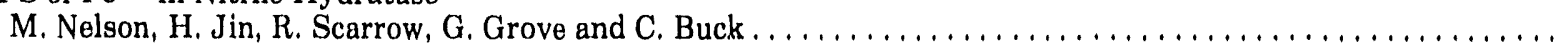

$\mathrm{X}$-ray Absorption Studies of Organo-Disulfide Redox Polymer Electrodes

X.Q. Yang, K.H. Xue, H.S. Lee, J. McBreen, T.A. Skotheim, and F. Iu . . . . . . . . . . . . . . . . . . . . .

\section{Beamline X19C}

X-ray Topographic Studies of the Phase Transformation Occuring at $-143^{\circ} \mathrm{C}$ in $p \cdot$ Terphenyl Single Crystals

R. DiSalvo, M. Dudley, J. Wu, and D. Gordon-Smith

Characterization of Chemomechanically Polished CdTe and CdZnTe Single Crystal Wafers by Synchrotron X-ray

White Beam Topography

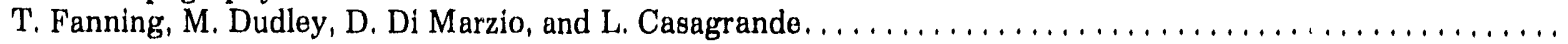

Modification of Precipitate Strain Fields Accompanying RTP of High Carbon Content Silicon

T. Fanning, M. Dudley, F.F.Y. Wang, D. Gordon-Smith, and D.T. Hodul. 
Observation of Combined Climb and Glide Induced by RTP of High Carbon Content Sillicon

T. Fanning, M. Dudley, F.F.Y. Wang, D. Gordon-Smith, and D.T, Hodul. ...

Observation of RTP Induced Dislocation Nucleation at Precipitates in High Carbon Content Sillicon

T. Fanning, M. Dudley, F.F.Y. Wang, D. Gordon-Smith, and D.T. Hodul..........................

Studies of RTP Induced Damage in High Carbon Doped Sillicon

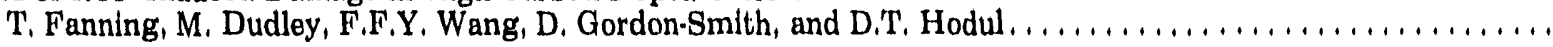

Characterization of Defect Structures Close to Grain Boundaries in Ice Crystals

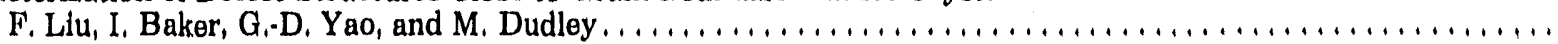

Observation of Faults Produced by Vacuncy Condensation in Ice Single Crystals

F. Liu, I, Baker, G.-D. Yau, and M. Dudley . . . . . . . . . . . . . . . . . . . . . . . . . . . . . . . . . . . . .

Synchrotron Topographic Investigation of Damage Distributions Resulting from Electromagnetic Breakdown of Epitaxial Sillicon p-n Junctions

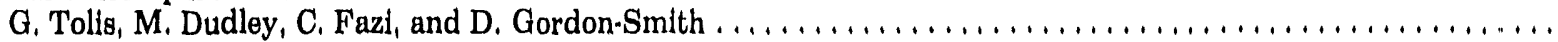

Assessment of Damage Induced by Electromagnetic Breakdown in Silicon B1-Polar Diodes by Synchrotron White

Beam X-ray Topography

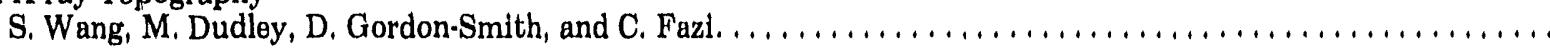

Grazing Bragg-Laue Synchrotron X-ray Topographic Imaging of Near-Surface Structural Damage in Silicon B1-Polar Diodes After Failure with Microwave Pulses

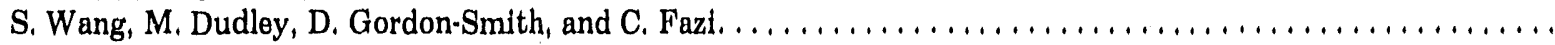

Characterization of Dislocation Structures Around Indents on $\{111\}$ Indium Antimonide Surfaces

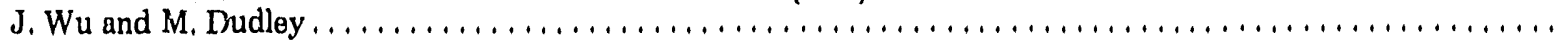

Characterization of Gel-Grown Crystals by Synchrotron White Beam Topography

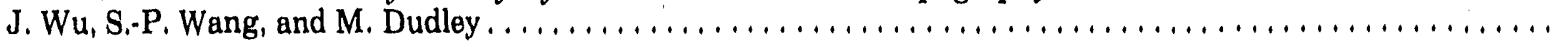

Analysis of Defect Structures in $\mathrm{NdGaO}_{3}$ Single Crystals

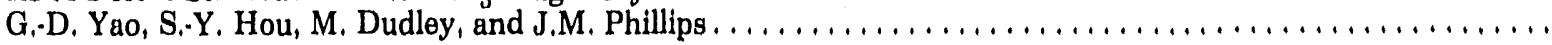

Analysis of Twin Structures in $\mathrm{LaAlO}_{3}$ Single Crystals

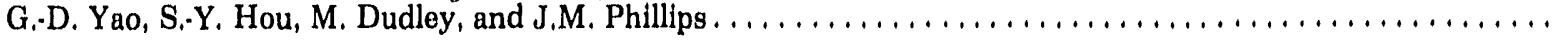

Comparison Between Observation and Calculation of Surface Relaxation Effects on Dislocation Strain Fields in

Thin Films

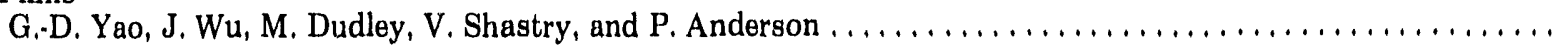

Imaging of Defect Structures in CdZnTe Single Crystals

G.-D. Yao, M. Dudley, T, Fanning, and D. Larson . . . . . . . . . . . . . . . . . . . . . . . . . . . . . .

Synchrotron Topography Observations of the Sec ond Order Phase Transition in Lanthanum Aluminate Single Crystals

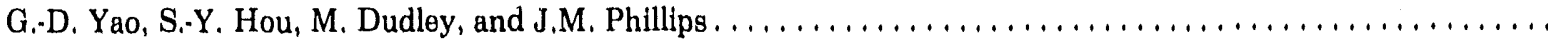

\section{Beamline X20A}

Observation of Enhanced Ordering in Polymer Films Near Air and Substrate Interfaces Using Grazing Incidence X-ray Diffraction

B.J. Factor, T.P. Russell, and M.F. Toney

Magnetic X-ray Scattering Study of the 3D Random Field Ising Model

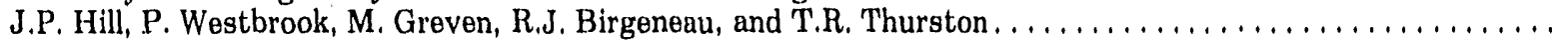

$\mathrm{X}$-ray Scattering Studies of Strain at the $\mathrm{SiO}_{\mathrm{x}} / \mathrm{Si}(001)$ Interface

S.D. Kosowsky, T.A. Rabedeau, P.S. Pershan, J. Bevk, and B.S. Freer......................... 247

Nitrogen Induced $(2 \times 3)$ Reconstruction of $\mathrm{Cu}(110)$

A.R. Sandy, C. You, S.G.J. Mochrie, A. Baddorf, D.M. Zehner, and L.D. Gibbs

Electrochemically Adsorbed Bismuth Monolayers on Silver (111): Surface Crystallography of the Uniaxially

Commensurate Structure

M.F. Toney, J.G. Gordon, M.G. Samant, G.L. Borges, D.G. Wiesler, D. Yee, and L.B. Sorensen. . . . . . . . . . .

Structural and Magnetic X-ray Scattering Measurements of Epitaxial Dy Thin Films

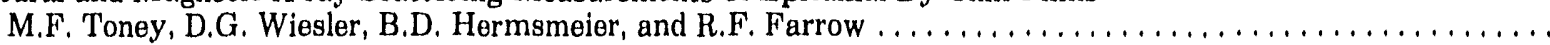

Underpotentially Deposited Thallium on Silver (111): Two-Dimensional Compressibility

M.F. Toney, J.G. Gordon, M.G. Samant, G.L. Borges, O.R. Melroy, D. Yee, and L.B. Sorensen 
Structure and Epitaxy of Anodic $1^{\prime} \mathrm{O}_{2} / \mathrm{Ti}(11 \mathrm{2} 0)$

Vol II

D.G. Wiesler, M.F, Toney, M. G. Samant, O.R. Melroy, W.H. Smyrl, and L.S. McMillan . . . . . . . . . . . . . . . . .

\section{Beamline X20B}

In-Situ X-ray Scattering Du.ing Electrodeposition Under Controlled Hydrodynamics

M.J. Armatrong, G.M. Whitney, and M.F. Toney

The Commensurate and Incommensurate Phases of Bllayer Xenon on Single Crystal Graphite

W.J, Nuttall, K.P. Fahey, M.J, Young, and R.J. Birgeneau . . . . . . . . . . . . . . . . . . . . . . . . . . .

\section{Beamline X20C}

Rapid Crystallization of Amorphous $\mathrm{CO}_{2} \mathrm{~B}$ Studied by Time Resolved X-ray Scattering

S. Brquer, H.E. Fischer, J.O. Ström.Olsen, M. Sutton and G.B. Stephenson . . . . . . . . . . . . . . . . . . . .

Polarization Measurements on a 3d Ising System

J.P. Hill, P. Westbrook, R.J. Birgeneau, T.R. Thurston, and D, Gibbs . . . . . . . . . . . . . . . . . . . . 251

Evidence for Two-Step Disordering of the $A u(110) 1 \times 2$ Reconstruction

D.T. Keane, P.A. Bancel, J.L. Jordan-Sweet, G.A. Held, A. Mak, and R.J. Birgeneau . . . . . . . . . . . . . . . . .

\section{Beamline X22B}

$\mathrm{X}$-Ray Resonance Magnetic Scattering in $\mathrm{Eu}\left(\mathrm{As}_{0.02} \mathrm{P}_{0.98}\right)_{3}$

T. Chattopadhyay, G. Grübel, and L. Rebelsky

X-ray Resonance Magnetic Scattering in EuTe

T. Chattopadhyay and G, Grübel

X-ray Reflectivity Study of Interface Growth Dynamics; In-Situ Sputter Deposition of Au on Silicon

R.P. Chiarello, H.K. Kim, and H. You ...................................

Resonant Magnetic Scattering In Europium Compounds

G. Grübel, J,D. Axe, L. Rebelsky, D. Gibbs and T. Chattopadhyay . . . . . . . . . . . . . . . . . . . .

Potential Induced $(1 \times 3)$ Reconstruction of the $\mathrm{Au}(110)$ Surface

B. M. Ocko, G. Helgesen, B. Schardt, J. Wang, and A. Hamelin .

X-ray Crystal Truncation Rod Analysis of Untwinned $\mathrm{YBa}_{2} \mathrm{Cu}_{3} \mathrm{O}_{7-\delta}$ Single Crystals: Termination Finger Print

H. You, U. Welp, G.W. Crabtree, Y. Fang, S.K. Sinha, J.D. Axe, X. Jiang, and S.C. Moss. . . . . . . . . . . . . . .

$\mathrm{X}$-ray Diffuse Scattering from Polybromostyrene PBrS/Toluene Solutions

W. Zhao, X. Zhao, M. Rafailovich, J. Sokolov, M. Sanyal, and S.K. Sinha . . . . . . . . . . . . . . . . . . .

\section{Beamline X22C}

Capillary Modes on a Close-Packed Metal Surface: Pt $(001)$

D.L. Abernathy, S.G.J. Mochrie, D.M. Zehner, G. Grübel, and D. Gibbs . . . . . . . . . . . . . . . . . . .

Order, Disorder and Structure of Crystals of $\mathrm{C}_{60} / \mathrm{C}_{70}$

J. Bohr, D. Gibbs, S.K. Sinha, W. Krätschmer, G. Van Tendeloo, E. Larson, H. Egsgaard, and

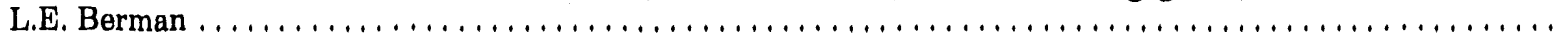

Magnetic X-ray Scattering Study of Tb

P.M. Gehring, L. Rebelsky, D. Gibbs, and G. Shirane $\ldots \ldots \ldots \ldots \ldots \ldots \ldots \ldots \ldots \ldots$

Reconstruction of the $\mathrm{Pt}(111)$ Surface

G. Grübel, K.G. Huang, D. Gibbs, D.M. Zehner, A.R. Sandy, and S.G.J. Mochrie. . . . . . . . . . . . . . . . 257

In-Plane Structural Modulation of the $\mathrm{CaF}_{2}$ Thin Film on Si(111)

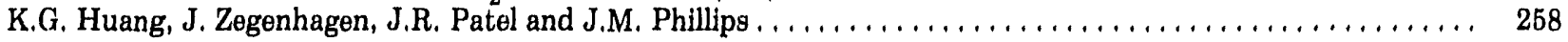

Specular and Diffuse Scattering from ErAs Buried Epitaxial Layers

P.F. Miceli, M. Sanyal, S.K. Sinha, and C.J. Palmstr $\phi m \ldots \ldots \ldots \ldots \ldots \ldots \ldots$

$\mathrm{Au}(113)$ Surface Studies

A.R. Sandy, D.L. Abernathy, S.G.J. Mochrie, D.M. Zehner, and L.D. Gibba

Anomalous Reflectivity Measurement of Electron Density Profiles in Thin Films M.K. Sanyal, S.K. Sinha, A. Gibaud, K.G. Huang, M. Rafailovich, J. Sokolov, X. Zhao, W. Zhao, and

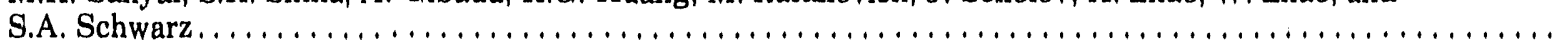


Determination of Electron Density Profile of a Thin Film Using Ariomalous Reflectivity

M.K. Sanyal, S.K. Sinha, A. Gibaud, K.G, Huang, B.L. Cau'valho, M. Rafallovich, J, Sokolov, X. Zhao,

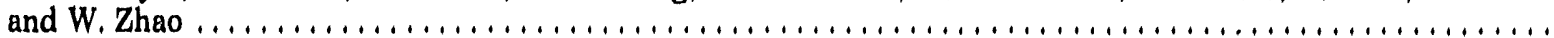

Resonance X-ray Magnetic Scattering Study of Erblum

M.K. Sanyal, D. Gibbs, J, Bohr, and M. Wulff . .

Magnetic Structure in $\mathrm{U}_{0.85} \mathrm{Th}_{0.15} \mathrm{Sb}$ Using Resonant X-ray Scattering

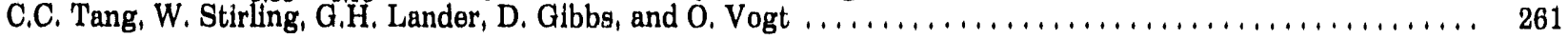

Magnetic Critical Scattering in Holmium

T.R. Thurston, D. Gibbs, J.P. Hill, and G. Shirane. . . . . . . . . . . . . . . . . . . . . . . . 261

X-ray Crystal Truncation Rod Analysis of Untwinned $\mathrm{YBa}_{2} \mathrm{Cu}_{3} \mathrm{O}_{\eta_{-} \delta}$ Single Crystals: Termination Finger Print

H. You, U. Welp, G.W. Crabtree, Y. Fang, S.K. Sinha, J.D. Axe, X. Jlang, and S.C. Moss. . . . . . . . . . . . . . . 255

X-ray Diffuse Scattering Study of Liquild Polymer Films

W. Zhao, X. Zhao, M. Rafallovich, J. Sokolov, M. Sanyal, and S.K. Sinha . . . . . . . . . . . . . . . . . .

\section{Beamline X23A2}

Spatially Selective Local Structural Information for InGaAs Multilayers Using Diffraction Anomalous Fine

Structure

C.E. Bouldin, J. Woiclk, H. Stragier, D. Yee, C. Bitz, L.B. Sorensen, and K. Kuhn . . . . . . . . . . . . . . . . . . .

Local Structural Determinations Using Diffraction Anomalous Fine Structure (DAFS) Measurements

J.O. Cross, H. Stragier, L.B. Sorensen, C.E. Bouldin, and J, Woicik . . . . . . . . . . . . . . . . . . . . . . . .

Atomic Exchange at Semiconductor Superlattice Interfaces

K.M. Kemner, B.A. Bunker, N. Samarth, H, Luo, and J.K. Furdyna. . . . . . . . . . . . . . . . . . . . . . . . . .

Total Electron Yield EXAPS at Low Temperatures

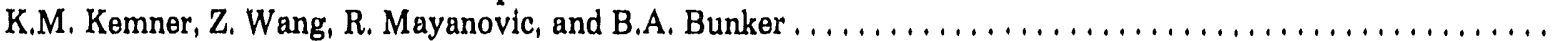

Study of Phase Transitions in Potassium Niobate

K.H. Kim, W.T. Elam, and M.I. Bell

Characterization of $\mathrm{A} \mathrm{Cu} / \mathrm{Al}_{2} \mathrm{O}_{3}$ Buried Interface Using Glancing-Angle X-ray Reflectivity and XAFS Studies

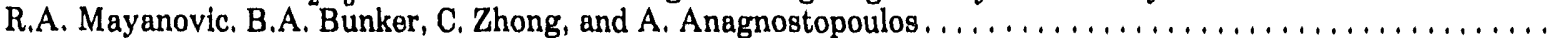

Glancing-Angle XAFS Studies of Premelting at $\mathrm{Cu} / \mathrm{Al}_{2} \mathrm{O}_{3}$ Interfacial Regions

R.A. Mayanovic, B.A. Bunker, and N. Jisrawi

Intermediate-Range-Order Around Copper in Crystalline and Amorphous Silicates Using EXAFS

D.A. McKeown

EXAFS Study of Structural Changes of the $\mathrm{Ni}_{3} \mathrm{Al}$ Ordered Intermetallic by Mechanical Milling

T. Nasu, A.M. Edwards, D.E. Sayers, and C.C. Koch

Orientation-Dependent XAFS Studies of High-' $\mathrm{T}_{\mathrm{c}}$ Superconducting $\mathrm{Pb}-\mathrm{Bi}-\mathrm{Sr}-\mathrm{Ca}-\mathrm{Cu}-\mathrm{O}$ system

W.F. Pong, P.K. Tseng, J.B. Shi, H.C. Ku, R.A. Mayanovic, and B.A. Bunker . . . . . . . . . . . . . . .

$\mathrm{X}$-ray Absorption Investigation of Amorphous Platinum Pyrimidine Derivatives

R. Serimaa, V, Etel †nlemi, T. Laitalainen, T, Paakkari, and I. Mutikainen.

Site Selective Local Structure Information for $\mathrm{YBaCuO}$ Using Diffraction Anomalous Fine Structure H. Stragier, C. Bitz, D. Yee, L.B. Sorensen, C.E. Bouldin, J. Woicik, J.M. Tranquada, and $\mathrm{K}$. Ludwig.

EXAFS Study of Spontaneous Ordering in $\mathrm{Ga}_{0.5} \mathrm{In}_{0.5} \mathrm{P}$ Thin Films Deposited on GaAs

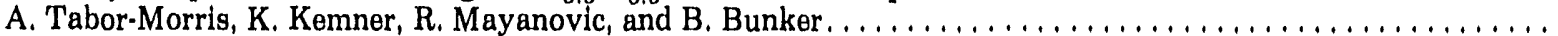

EXAFS Study of Gold-Induced Germanium Crystallization

Z. Tan, S.M. Heald, C.E. Bouldin, and J.C. Woick.

EXAFS Study of Ferroelectric $\mathrm{PbS}_{\mathbf{x}} \mathrm{Te}_{1-\mathrm{x}}$ Alloys

Z. Wang and B.A. Bunker.

EXAFS Study of the Ferroelectric $\mathrm{Zn}_{\mathrm{x}} \mathrm{Cd}_{1-\mathrm{x}}$ Te Alloys

Z. Wang, R. Mayanovic, B. Bunker, U. Debska, and J. Furdyna 
In-Situ Observation of Defect Formation in CdT Crystals at High Temperatures Due to Imposed Mechanical Stresses

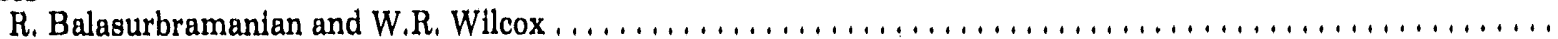

Diffraction Imaging of a Homoepitaxial Dlamond Film

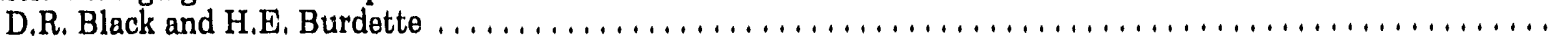

Diffraction Imaging of Isotopically Pure Diamond

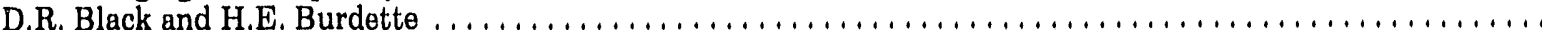

Diffraction Imaging of Isotopically Pure Man-Made Diamond

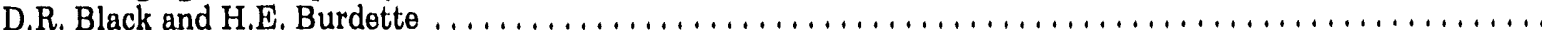

Absolute Calibration of Small-Angle X-ray Scattering

R.R. Jemian, G.G. Long, S. Krueger, and D.R. Black

Morphology of L-Arginine-Phosphate Crystals for Nonlinear Optical Devices

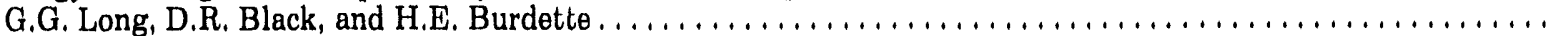

$\mathrm{X}$-ray Diffraction Imaging of L-Arginine Phosphate (LAP)

G.G. Long, D.R. Black, H.E. Burdette, S.M. Rao, and R.B. Lal . . . . . . . . . . . . . . . . . . . . . . . .

Structure of Microporous Foams

G.G. Long, S. Krueger, B.J. Olivier, R. Lagasse and D.W. Schaefer . . . . . . . . . . . . . . . . . . . . .

High-Resolution SAXS Study of the Structure of Microporous Foams

B.J. Olivier, D.W. Schaefer, S. Krueger, and G. Long

Diffraction Topography and High Resolution Diffraction Microscopy of Lattice Imperfections, Impurity Inclusions and Fracture in Amethstine $\alpha$-Quartz

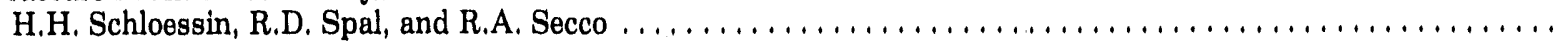

Phase Contrast Fresnel Diffraction of Hard X-rays by a Thin Film Edge

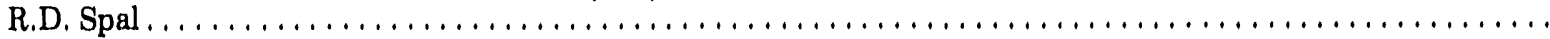

The Effect of Microgravity on the Mesoscopic Structure of Lead Tin Tellluride: High Resolution Monochromatic Diffraction Imaging of Crystals Grown in Microgravity on Space Shuttle STS 61A and on the Ground

B. Steiner, R.C. Dobbyn, D. Black, H. Burdette, M. Kuriyama, R. Spal, R. Simchick, and A. Fripp

The Mesoscopic Structural Transition from Czochralski to Bridgman Growth of Gallium Arsenide: High Resolution Monochromatic Diffraction Imaging

B. Steiner, R.C. Dobbyn, D. Black, H. Burdette, M. Kuriyama, R. Spal, D. Matthiesen, and B. Ditchek ........

The Mesoscopic Structure of Mercuric Iodide Crystals Grown in Spacelab III and on the Ground: High Resolution Monochromatic Diffraction Imaging

B. Steiner, R.C. Dobbyn, D. Black, H. Burdetto, M. Kuriyama, R. Spal, and L. van den Berg

Structural Continuity in Triglycine Sulfate Crystals Grown in Spacelab III: High Resolution Monochromatic

Diffraction Imaging

B. Steiner, R.C. Dobbyn, D. Black, H. Burdette, M. Kuriyama, R. Spal, R.B. Lal, and A. Batra

\section{Beamline X23B}

$\mathrm{Ag} \mathrm{L} \mathrm{L}_{2,3}$ Edge XANES Study of Charge Redistribution at the Ag Site in Au-Ag Alloys

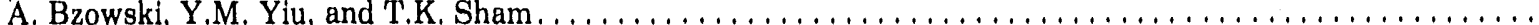

Structure in Multilayer Systems

C.I. Foiles, J.A. Cowen, D. Koppel, H.-K, Sung, and T.I. Morrison

EXAFS Studies of Structural Anisotropy in Fe-Tb Amorphous Films

V.G. Harris, K.D. Aylesworth, N.C. Koon, and W.T. Elam .

EXAFS Studies of Amorphous-To-Crystalline Transition in $\mathrm{Co}_{74} \mathrm{Fe}_{6} \mathrm{~B}_{15} \mathrm{Si}_{5}$ Thin Film Specimens

V.G. Harris, S.A. Oliver, P. Dorsey, C. Vittoria, K.H. Kim, and W.T. Elam . . . . . . . . . . . . . . . . . .

EXAFS Studies of IBS Fe-Tb Alloy Films

V.G. Harris, K.H. Kim, and W.T. Elam.

Fe Thickness Effect on Structure and Magnetism in Low-Dimesional Fe/Tb Multilayered Structures

V.G. Harris, K.H. Kim, N.C. Koon, W.T. Elam, R. Coehoorn, and W. Hoving . . . . . . . . . . . . . . . . . . . . 
X-24A Experimental Techniques: Flux Measurements from Grating-Multilayer Monochromator Combinations for Low Energy Studies

T. Jach, S. Southworth, P.L. Cowan, R.C.C. Perera, and B.A. Karlin. . . . . . . . . . . . . . . . . . . . .

$\mathrm{X}$-ray Adsorption Isotherms

J.L. Jordan-Sweet, B.D. Swanson, and L.B. Sorensen

X-ray, Soft X-ray, and VUV Beam Position Monitor

B.A. Karlin, P.L. Cowan, and J.C. Woicik

X-ray Standing-Wave Study of Sb on $\operatorname{InP}(110), \mathrm{GaP}(110)$ and $\mathrm{GaAs}(110)$

T. Kendelewicz, J.C. Woicik, K.E. Miyano, P.L. Cowan, E.A. Karlin, C.E. Bouldin, P. Pianetta, and

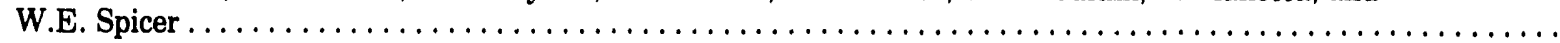

Double Photoionization of Helium at $2.8 \mathrm{keV}$

J.C. Levin, D.W. Lindle, N. Keller, R.D. Miller, Y. Azuma, N.B. Mansour, H.G. Berry, and I.A. Sellin .........

X-ray Beam Position Monitor Using A Quadrant Pin Diode

S.H. Southworth and P.L. Cowan

X-ray Standing-Wavn Study of Rare Gas Atoms Physisorbed on Single Crystal Grephite

B.D. Swanson, C. Bitz, D. Yee, L.B. Sorensen, J. Jordan-Sweet, P. Cowan, and B. Karlin

Determination of the $\mathrm{Sb} / \mathrm{Si}(111)$ Interfaciel Structure by Back Reflection X-ray Standing Waves and Surface

Extended X-ray Absorption Fine Structure

J.C. Woicik, T. Kendelewicz, K.E. Miyano, P.L. Cowan, C.E. Bouldin, B.A. Karlin, P. Pianetta, and

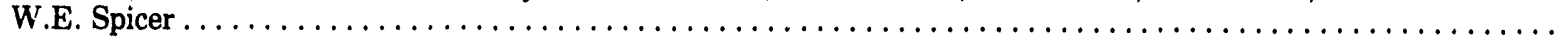

X-ray Standing Wave Determination of the Clean InP(110) Surface Reconstruction

J.C. Woicik, T. Kendelewicz, K.E. Miyano, P.L. Cowan, C.E. Bouldin, B.A. Karlin, P. Pianetta, and

W.E. Spicer .

\section{Beamline X24C}

Efficiency of Soft X-ray Detector Phosphors

E.L. Benitez, D.E. Husk, and S.E. Schnatterly

Photoemission Study of Hydrogen Adsorption on Vanadium Dioxide Near the Semiconductor - Metal Phase

Transition

V.M. Bermudez, R.T. Williams, J.P. Long, R.K. Reed, and P.H. Klein

Combined Pulsed Laser and Synchrotron Radiation Measurements of Photochemical Decomposition of GaAs(110)

J.P. Long, S.S. Goldenberg, and M.N. Kabler.

Photoelectron Spectroscopy of Semiconductors Exhibiting Surface-Poteniial Inhomogeneities

J.P. Long, S.S. Goldenberg, and M.N. Kabler

C-1s NEXAFS and EXAFS of Graphite, Natural Diamond, and Synthetic Diamond Thin Films

J. Nithianandam and J.C. Rife

Process-Dependent Spectral Reflectance Studies of Thermal $\mathrm{SiO}_{2}$ on $\mathrm{Si}$

H.F. Wei, A.K. Henning, T. Hochwitz, J. Slinkman, J. Rife, and W.R. Hunter

\section{Beamline X25}

Performance of an Adaptive Jet Cooled Silicor Monochromator in a High Power Density X-ray Beam

L.E. Berman, M. Hart, and S. Sharma.

Order, Disorder and Structure of Crystals of $\mathrm{C}_{60} / \mathrm{C}_{70}$

J. Bohr, D. Gibbs, S.K. Sinha, W. Krätschmer, G. Van Tendeloo, E. Larsen, H. Egsgaard,

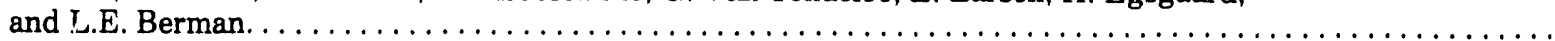

Performance of Be Crystals in a High Power Density X-ray Beam

A.K. Freund, S. Joksch, K. Kawata, G. Marot, E. Ziegler, L.E. Berman, and J.B. Hastings . . . . . . . . . . . . .

Performance of Very Thin Si Crystals Under High Heat Load

A.K. Freund, G. Marot, K. Kawata, S. Joksch, E. Ziegler, L.E. Berman, and J.B. Hastings . . . . . . . . . . . .

Inelastic X-ray Scattering Spectroscopy in Aluminum

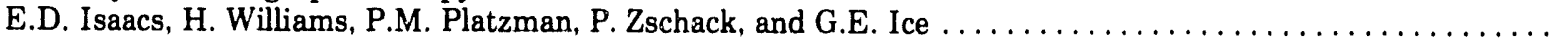


High Resolution X-ray Absorption Spectroscopy

$\underline{\text { Vol II }}$

K. Hämäläinen, D.P. Siddons, J.B. Hastings, L.E. Berman, C.-C. Kao, V. Stojanoff,

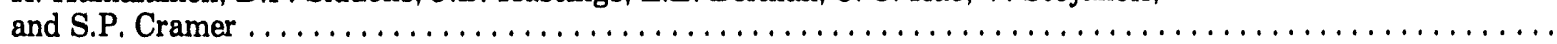

Performance of a Gallium Cooled $85^{\circ}$ Inclined Silicon Monochromator for a High Power Density X-ray Beam

W.K. Lee, A.T. Macrander, D.M. Mills, C.S. Rogers, R.K. Smither, and L.E. Berman ...................

High Resolution Inelastic X-ray Scattering Study of the Boron K-Absorption Edge

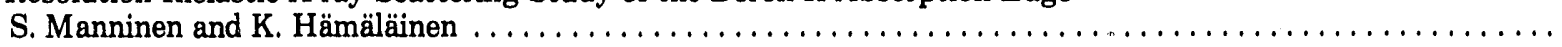

291

291

Cryogenic Cooling of Si Monochromators

G. Marot, A.K. Freund, M. Rossat, S. Joksch, K. Kawata, E. Ziegler, L.E. Berman,

D. Chapman, J.B. Hastings, M. Iarocci, G. van der Laske, and N. Lazarz $\ldots \ldots \ldots \ldots \ldots \ldots \ldots \ldots \ldots \ldots$

Kinetics of the Electrochemical Induced Reconstruction of che Au(111) Surface

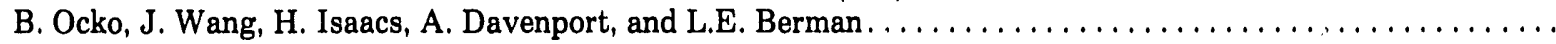

Potential Induced $(1 \times 3)$ Reconstruction of the $A u(110)$ Surface

B.M. Ocko, G. Helgesen, B. Schardt, J. Wang, and A. Hamelin .

A Comparison of High-Resolution Tryspin Crystal Structures Obtained by White-Beam Laue and Monochromatic

X-ray Diffraction Using Synchrotron Radiation

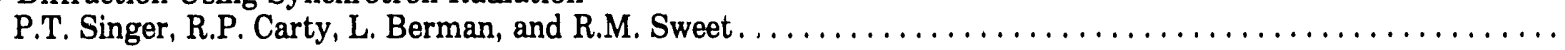

Intensity Fluctuation Spectroscopy Using Coherent X-rays

M. Sutton, E. Dufresne, S.G.J. Mochrie, L.E. Berman, G. Held, and G.B. Stephenson . . . . . . . . . . . .

$\mathrm{X}$-ray Search for CDW Satellites in $\mathrm{Ba}_{1-\mathrm{x}} \mathrm{K}_{\mathrm{X}} \mathrm{BiO}_{3}$

P. Wochner, Q.J. Wang, S.C. Moss, S.K. Sinha, G. Grübel, H. Chou, L.E. Berman, J.D. Axe,

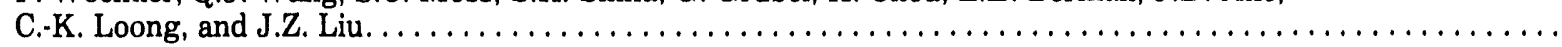

204

293

High Heat Load Study of Multilayer Monochromators at Room and Low Temperatures

E. Ziegler, A.K. Freund, G. Marot, S. Joksch, K. Kawata, M. Rossat, L.E. Berman, J.B. Hastings,

M. Iarocci, G. van der Laske, and N. Lazarz . . . . . . . . . . . . . . . . . . . . . . . . . . . . . . . . . .

294

\section{Beamline X26A}

Lunar Volcanic Glasses: Trace Element Abundances in Individual Spheres

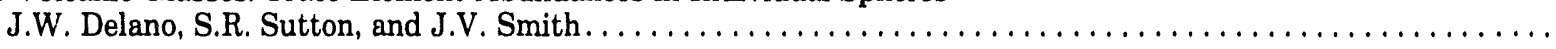

Detection and Quantitation by SRXRF of Some Trace Metals in Corn Bran and Corn Bran Passed Through the Pig

Gastrointestinal Tract

F. Dintiz and K. Jones .

Cosmic Class Stratospheric Particles: Trace Elements and Zn Depletions

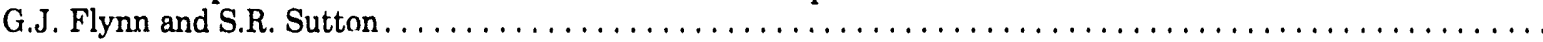

Identification and Quantification of Toxic Trace Elements in Mid-Continental Atmospheric Aerosols

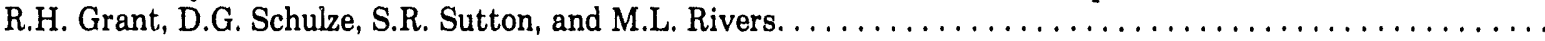

Chromium Oxidation State in Individual Lunar Olivine Grains Using MicroXANES

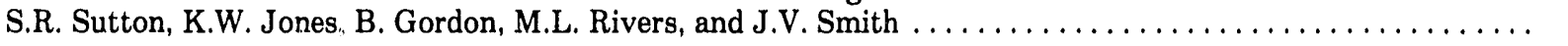

Experimental Determination of the X26 Bending Magnet X-ray Source Size

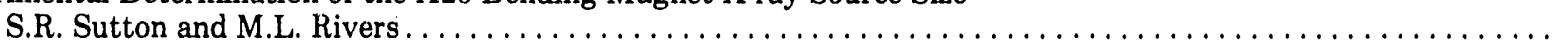

Analysis of Oceanic Fluid Inclusions by Synchrotron X-ray Fluorescence

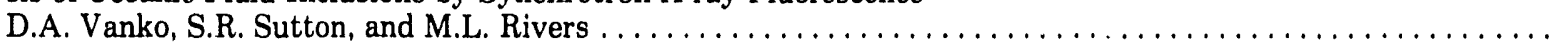

Analysis of Synthetic Fluid Inclusions in Quartz by SXRF Microbe

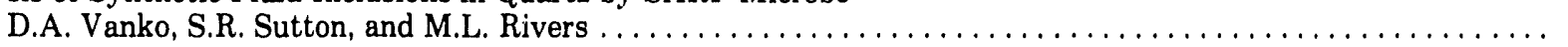

\section{Beamline X26C}

Analysis of Titanium in Boron Nitride Coating on a Beryllium Window

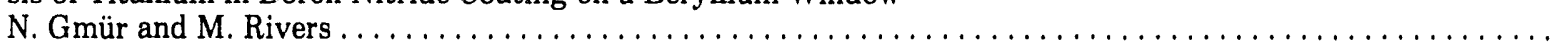

Measurements of Compton-Ejected Electrons for Ne Gas Using Filtered "White Light" of 10-15 keV

N.A. Guardala, J.L. Price, M.F. Stumborg, D.G. Simons, G.A. Glass, Jr., B.M. Johnson,

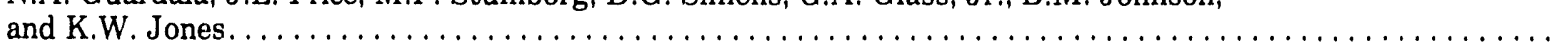


Development of Wire Scanner to Determine Vertical Profiles of Intersecting Photon and Ion Beams

Synchrotron X-ray Fluoresence Microanalysis of Synthetic Calcite Single Crystals

J. Paquette

Dynamic Studies of High-Resolution Tryspin Crystal Structures During Catalysis Obtained by White-Beam Laue

$\mathrm{X}$-ray Diffraction Using Synchrotron Radiation

P.T. Singer, R.P. Carty, K. Jones, W.F. Mangel, and R.M. Sweet .

Laue Diffraction Studies at X26C

E. Westbrook, M. Westbrook, I. Schlichting, D. Ringe, K.W. Jones, P. Singer, P. Spanne, and R. Sweet

Experimental Characterization of Circular Fresnel Zone Plates

W. Yun, J. Chrzas, B. Lai, D. Legnini, J. Viccaro, R. Bionta, and K. Skulina . 


\section{NSLS USERS' PUBLICATION LIST**}

\section{Beamline U1}

Hayes, R. G., Eberhardt, W., "Electron-Ion Coincidences Studies of the Fragmentation of Thiophene and of Tetrahydrothiophene Upon Core Ionization", J. Chem. Phys. 94 (1), 397, (1991).

Hayes, R. G., Eberhardt, W., "Electron-Ion Coincidence Studies of the Behaviour of Molecules Upon Core Excitation", Physica Scripta, 41, 449-453, (1990).

Parker, D. H., Fischer, D. A., Colbert, J., Koel, B. E., and Gland, J. L., "Hydrogen Induced Low Temperature Co Displacement from Pt(111) Surface", Surf. Sci. Lett. 236, L372-L376, (1990).

Yang, C. Y., Fischer, D. A., (et al) in High Temperature Super Conductors: Fundamental Properties and Novel Material Processing edit by D. Christer, J. Narayan and L. Schneemeyer. "The Effects of Metal Doping: $\mathrm{YBa}_{2}\left(\mathrm{Cu}_{1-\mathrm{x}} \mathrm{M}_{\mathrm{x}}\right)_{3} \mathrm{O}_{7-\delta}(\mathrm{M}=\mathrm{Fe}, \mathrm{Co}$, Ni, Zn)", MRS. symp. Vol. 169, 225-228, (1990).

\section{Beamline U3C}

Dessau, D. S., Shen, Z.X., Wells, B. O., Spicer, W. E., List, R. S., Arko, A. J., Bartlett, R. J., Fisk, Z., Cheong, S.-W., Olson, C. G., Mitzi, D. B., Eom, C. B., Kapitulnik, A., Geballe, T. H. and Scriber, J. E., "The Gold/High Temperature Superconductor Interface: Metallicity of the Near Surface Region and a Search for the Proximity Effect",J. Vac. Sci. and Tech. A9, 383, (1991).

Dessau, D. S., Wells, B. O., Shen, Z.-X., Spicer, W. E., Arko, A. J., List, R. S., Mitzi, D. B. and Kapitulnik, A., "Anomalous Spectral Weight Transfer at the Superconducting Transition of $\mathrm{Bi}_{2} \mathrm{Sr}_{2} \mathrm{CaCu}_{2} \mathrm{O}_{8+\delta}$ ", Phys. Rev. Lett. 66, 2160, (1991).

Spindt, C. J., Yamada, M., Meissner, P. L., Miyano, K. E., Herrera, A., Speicer, W. E., Arko, A. J., Woodall, J. M. and Pettit, G. D., "Au and A1 Schottky Barrier Formation on GaAs(100) Surfaces Prepared by Thermal Desorption of a Protective Arsenic Coating", J. Vac. Sci. and Tech. B9, 2090, (1991).

Shen, Z.-X, List, R. S., Arko, A. J., Bartlett, R. J., Dessau, D. S., Wells, B. O., Jepsen, O., Shih, C. K., Parmigiani, F., Huang, J. C., Lindberg, P. A. P., Lindau, I., Spicer W. E., "Electronic Structure of NiO - Correlaton and Band Effects", Phys. Rev. B 44, 3604, (1991).

\section{Beamline U4A}

Elliot, G. S., Smith, K. E. and Kevan, S. D., "Surface Localized States and The Fermi Contours of Pd(001)", Phys. Rev. B 43, 3893 (1991).

Riffe, D. M., Wertheim, G. K., and Citrin, P. H., "Enhanced Vibrational Broadening of Core-Level Photoemission from the Surface of $\mathrm{Na}(110) "$, Phys. Rev. Lett. 67, 116, (1991).

Rowe, J. E., Wertheim, G. K. and Riffe, D. M., "Silicon 2p Surface Core-Level Line Shape Si(111)-B", J. Vac. Sci. Tech. A9, 1020, (1991).

Smith, K. E., Elliot, G. S. and Kevan, S. D., "Reconstruction and Surface Fermi Surface of W(001)", Phys. Rev. B 42, 5385, (1990).

Smith, K. E. and Kevan, S. D., "Electronic Effects in the Mo(001) Surface Reconstruction: Two-dimensional Fermi Surfaces and Non-adiabaticity", Phys. Rev. B 43, 3986, (1991).

Smith K. E. and Kevan, S. D., "Electronic Structure of $(1 \times 1)$ H/Mo(001): Two-dimensional Fermi Surface and Non-adiabatic Adsorbate Vibrational Damping", Phys. Rev. B 43, 1831, (1991).

Smith K. E. and Kevan, S. D., "The Role of Non-adiabaticity and Two-dimensional Fermi Surfaces in the Reconstruction of Mo(001) and W(001)", Appl. Surface Sci. 49/49, 135, (1991).

Wei, D., Smith K. E. and Kevan, S. D., "Surface Fermi Contour and Phonon Anomalies on Pt(111)", Phys. Rev. B 43, 12062, (1991).

Wertheim, G. K., Riffe, D. M., Rowe, J. E. and Citrin, P. H., "Crystal-Field Splitting and Charge Flow in the Buckled-Dimer Reconstruction of Si(100)-2×1", Phys. Rev. Lett. 67, 120, (1991).

Wertheim, G. K., Rowe, J. E., Riffe, D. M. and Smith, N. V., "Improved Resolution of a 6m Toroidal Grating Monochromator", AIP Conf. Proc. 215, edited by T. A. Carlson, M. O. Krause, and S. T. Mason, (American Institute of Physics, New York, 1990) p. 259.

\footnotetext{
*Publications list was complied from the PRT/IDT progress reports for the period 9/16/90 through 9/15/91. It contains only published articles, no abstracts, and is not a complete listing for the facility.
} 


\section{Beamline U4B}

Abbate, M., deGroot, F. M. F., Fuggle, J. C., Ma, Y. J., Chen, C. T., Sette, F., Shin, S. and Fujimori, A., "Soft X-Ray Absorption Studies of the Electronic Structure Changes Through the $\mathrm{VO}_{2}$ Phase Transition", Phys. Rev. 43, (1991).

Chen, C. T., Sette, F., Md, Y., Hybertsen, M. S., Stechel, E. B., Foulkes, W. M. C., Schluter, M. A., Cheong, S-W., Cooper, A. S., Rupp, L. W., Jr., Batlogg, B., Soo, Y. L., Ming, Z. H., Krol, A. and Kao, Y. H., "Electronic States in Laa $2-\chi \mathrm{Sr}_{\chi} \mathrm{CuO}_{4+\delta}$ Probed by Soft X-ray Adsorption", Phys. Rev. Lett. 66, 104 (1991).

Chen, C. T., Sette, F., Ma Y. and Modesti, S., "Soft X-Ray Magnetic Circular Dichroism at the $\mathrm{L}_{2,3}$ Edges of Nickel", Phys. Rev. B42, 7262 (1990).

Chen, C. T., Sette, F, and Smith, N. V., "A Double Headed Dragon Monochromator for Soft X-ray Circular Dichroism Studies", Applied Optics 29, 4535, (1990).

Chen, C. T., Smith, N. V. and Sette, F., "Exchange, Spin-Orbit and Correlation Effects in the Soft X-Ray Magnetic-CircularDichroism Spectrum of Nickel", Phys. Rev. B43, 6785, (1991).

Gadea, F. X., Köppel, H., Schirer, J., Cederbaum, L. S., Randoll, K. J., Bradshaw, A. M., Ma, Y., Sette F. and Chen, C. T., "Vibronic Coupling and Core-hole Localization in K-shell Excitations of Ethylene", Phys. Rev. Lett. 66, 883, (1991).

Ma, Y., Chen, C. T., Meigs, G., Randall K. J. and Sette, F., "High Resolution Inner-Shell Photoabsorption Measurements of Simple Molecules in X-Ray and Inner-shell Processes", edited by T. A. Carlson, M. O. Krause and S. T. Manson, AIP Conference Proceedings 215, 634, (1990).

Ma, Y., Rowe, J. E., Chaban, E. E., Chen, C. T., Headrick, R. L., Meigs, S., Modesti, and Sette, F., "Surface States and Alkulito-Ser...iconductor Charge Transfer of the K/Si(111) $(\sqrt{3} \times \sqrt{3}) \mathrm{R}\left(30^{\circ}\right)$-B System", Phys. Rev. Lett. 17, 2173, (1990).

\section{Beamline U4IR}

Carr, G. L., Quijada, M., Tanner, D. B., Hirschmugl, C. J., Williams, G. P., Etemad, S., DeRosa, F., Venkatesan, T., Dutta, B., Hemmick, D., Xi, X, "Infrared Response of $\mathrm{YBa}_{2} \mathrm{Cu}_{3} \mathrm{O}_{7-\delta}$ Filma to Pulsed, Broadband Synchrotron Radiation", SPIE 1292, 187, (1990), BNL 44573.

Carr, G. L., Quijada, M., Tanner, D. B., Hirschmugl, C. J., Williams, G. P., Etemad, S., Dutta, B., DeRosa, F., Venkatesan, T., "Fast Bolometric Response by High T $\mathrm{c}$ Detectors Measured with Subnanosecond Synchrotron Radiation", Appl. Phys. Lett., 57, 2725 (1990) BNL 45529.

Forro, L., Carr, G. L., Williams, G. P., Mandrus, D., Mihaly, L., "Far Infrared Transmission Study of Single Crystal $\mathrm{Bi}_{2} \mathrm{Sr}_{2} \mathrm{Ca}_{1} \mathrm{Cu}_{2} \mathrm{O}_{\mathrm{x}}$ Superconductors", Phys. Rev. Lett. 65, 1941, (1990), BNL 45203.

Gao, F., Carr, G. L., Porter, C. D., Tanner, D. B., Etemad, S., Venkatesan, T., Inam, A., Dutta, B., Wu, X. D., Williams, G. P., Hirschmugl, C. J., "Far Infrared Transmission and Reflection Studies of Oriented $\mathrm{YBa}_{2} \mathrm{Cu}_{3} \mathrm{O}_{7-\delta}$ Thin Films", Phys. Rev. B43, 10383, (1991), BNL 45254.

Hirschmugl, C. J., Williams, G. P., Hoffmann, F. M., Chabal, Y. J., "Adsorbate-Substrate Resonant Interactions Observed for $\mathrm{CO}$ on $\mathrm{Cu}(100)$ and (111) in the Far-IR Using Synchrotron Radiation", J. of Elec. Spec. \& Relat. Phenomena, 54/55, 109, (1990), BNL 45253.

Hirschmugl, C. J., Sagurton, M., Williams, G. P., "Multiparticle Coherence Calculations for Synchrotron Radiation Emission", Phys. Rev. A, 44, 1316, (1991). BNL 46117.

Romero, D. B., Carr, G. L., Tanner, D. B., Forro, L., Mandrus, D., Mihaly, L., Williams, G. P., "12k $\mathrm{B}_{\mathrm{c}}$ Optical Signature of Superconductivity in Single-Domain $\mathrm{Bi}_{2} \mathrm{Sr}_{2} \mathrm{CaCu}_{2} \mathrm{O}_{8}$ ", Phys. Rev. B, 44, 2818, (1991), BNL 45118.

Williams, G. P., "The Initial Scientific Program at the NSLS Infrared Beamline", Nucl. Instrum. and Methods A291, 8, (1990).

\section{Beamline U5U}

Brookes, N. B., Chang Y. and Johnson, P. D., "Magnetic Interface States and Finite-Size Effects", Phys. Rev. Lett. 67, 354, (1991).

\section{Beamline U6}

Boyne, D., Hsia, L., Wachnik, R., Decker, R. and Wasik, C., "X-ray Radiation Damage and a Reliability Study on Bipolar Devices", Appl. Phys. Lett. 58, 2687, (1991).

Conley, W., Dundatscheck, R., Gelorme, J., Horvat, J., Martino, R., Murphy, E., Petrosky, A., Spinillo, G., Stewart, K., Wilbarg, R. and Wood, R., "Chemically Amplified Negative Tone Photoresist for Sub-Half Micron Device and Mask Fabrication", Proc. SPIE 1466, 39, (1991). 
Dunn, G., "Effect of Synchrotron X-r'ay Radiation on the Channel Hot-Carrier Reliability of Reoxidized Nitrided Silicon Dioxide", IEEE Electron Device Letters 12, 8, (1991).

Hsia, L. C., "A Study of X-ray Damage Effects on Open-Bottom Trench Isolation for Bipolar Devices", J. Electrochem. Soc. 138, 239, (1991).

Maldonado, J. R., "X-ray Lithography System Development at IBM: Overview and Status", Proc. SPIE 1465, 2, (1991).

Wagner, A., Levin, J. P., Mauer, J. L., Blauner, P. G., Kirch S. J. and Longo, P., "X-ray Mask Repair With Focused Ion Beams", J. Vac. Sci. Tech. B8, 1557, (1990).

\section{Beamline U7}

Braaten, N. A., Borg, A., Grepstad, J. K., Raaen, S. and Ruckman, M. W., "Oxygen K-edge Structure for Thin Ce Oxide Films", Solid State Comm. 77, 731-4, (1991).

Horache, E., Fischer, J. E., Ruckman, M. W., "Resonant Photoemission Study of Ternary Transition Metal Silicides", Phys. Rev. B 42, 11079-85, (1990).

Jeon, Y., Liang, G., Chen, J., Croft, M., Ruckman, M. W., DiMarzio, D., Hegde, M. S., "A Study of BaBiO 3 and the Effect of Potassium Substitution Using Photoemission", Phys, Rev. B 41, 4066-71, (1990).

Li, H. Tian, D., Quinn, J., Li, Y. S., Jona, F. and Marcus, P. M., "Low-energy Electron Diffraction and Photoemission Study of Epitaxial Films of $\mathrm{Cu}$ on $\mathrm{Ag}(001)$ ", Phys. Rev. B 43,6342-6, (1991).

Li. H., Wu, S. C., Tian, D., Li, Y.S., Quinn, J., and Jona, F., "Ultrathin Films of Rh on Au(001) and Rh on Ag(001): Growth Mode and Magnetism", Phys, Rev, B 44, 1438-41, (1991).

Lin, C. L., Qiu, S. L., Chen, Jie, Tranquada, J. M., and Strongin, M., "X-ray Absorption Studies of $\mathrm{Nd}_{2-\mathrm{x}} \mathrm{Ce}_{\mathrm{x}} \mathrm{CuO}_{4}$ ", Phys. Rev. $B$ 42, 4763-66, (1990).

Qiu, S. L., Lin, C. L., Chen, Jie, and Strongin, M., "The Formation of Metal Oxygen Species at Low Temperatures", J. Vac. Sci. Tech. A8, 2595-98, (1990).

Qiu, S. L., Ruckman, M. W., and Strongin, M., "Near-edge and Photoemission Studies of the Interaction of Cesium and Ammonia at $80 \mathrm{~K}$ ", J. Vac. Sci. Tech. A 9, 1645-9, (1991).

Quinn, J., Li, Y. S., Li, H., Tian, D., Jona, F. and Marcus, P. M., "Atomic and Electronic Structure of Fe Films Grown on Pd(100)", Phys. Rev. B 43, 3959-68, (1991).

Park, K. T., Cao, J., Gao, Y., Wicks, G. W., and Ruckman, M. W., "Growth of Al Nitride Layers on GaAs(100) by Reaction With Condensed Ammonia", J. Appl. Phys. 70, 2623-7, (1991).

Raaen, S., Braaten, N. A., Grepstad, J. K., and Qiu, S. L., "Enhanced Oxidation of Aluminum: Effects of Thin Cerium Overlayers", Physica Scripta 41, 1001-04, (1990).

Ruckman, M. W., Cao, J., Park, K. T., and Gao, Y., "Photon-assisted Nitridation of GaAs(100) at Liquid Nitrogen Temperature", Appl. Phys. Lett. 59, 849-51, (1991).

Ruckman, M. W., Qiu, S. L., Chen, J., and Strongin, M., "Near-edge Study of Molecular Oxygen and Nitrogen Clusters on a Krypton Surface", Phys. Rev. B 43, 8603-5, (1991).

Ruckman, M. W., Qiu, S. L., Strongin, M., "AC Detection of Secondary Photoelectrons", Rev. Sci. Instrum. 62, 1113-14, (1991).

Wu, S. C., Li, H., Tian, D., Quinn, J., Li, Y. S., Sokolov, J., Jona, F. and Christensen, "Electronic Band Structure of a Rare Earth Metal: Tb(0001)", Phys. Rev. B 41, 11911-18, (1990).

Yang, X. Q., Ruckman, M. W., Skotheim, T. A., den Boer, M., Zheng, Y., Badzian, A. R., Badzian, T., Messier, R., Srivatsa, A. R., "X-ray Absorption Study of Diamond Films Grown by Chemical Vapor Deposition", J. Vac. Sci. Tech. A9, 1140-4, (1991).

\section{Beamline U8}

Barton, J. J., Himpsel, F. J. "Photoelectron Spectroscopy", Proceedings of the Enrico Fermi School on Photoemission and Absorption Spectroscopy of Solids and Interfaces with Synchrotron Radiation, ed. by M. Campagna and R. Rosei, North Holland, p. 429, (Amsterdam 1990).

Didziulis, S. V., Lince, J. R., Fleischauer, P. D. and Yarmoff, J. A., "Photoelectron Spectroscopic Studies of the Electronic Structure of $\alpha^{-} \mathrm{SiC}^{\prime}$, Inorganic Chemistry 30, 672, (1991).

Eastman, D. E., Himpsel, F. J., "Photoelectron Spectroscopy", Encyclopedia of Physics, ed. by R. G. Lerner and G. L. Trigg, second edition, VCH Publishers, p. 908, (New York 1991). 
Gloub, L., Herant, M., Kalata, K., Lovas, I., Nystrom, G., Spiller, E. and Wilczynski, J., "Sub Arcsecond Observations of the Solar X-ray Corona", Nature 344, 842-844, (1990).

Himpsel, F. J., "Inverse Photoemission from Semiconductors", Surf. Sci Reports 12, 1, (1990).

Himpsel, F. J., Meyerson, B. S., McFeely, F. R., Morar, J, F., Taleb-Ibrahimi, A. and Yarmoff, J. A., "Core Level Spectroscopy at Silicon Surfaces and Interfaces", Proceedings of the Enrico Fermi School on Photoemission and Absorption Spectroscopy of Solids and Interfaces with Synchrotron Radiation, ed. by M. Campagna and R. Rosei, North Holland, p. 203, (Amsterdam 1990).

Lince, J. R., Didziulis, S. V. and Yarmoff, J. A., "Resonant Photoelectron Spectroscopy Study at the Mo 4p $\rightarrow 4 \mathrm{~d}$ Absorption Edge in $\mathrm{MoS}_{2}{ }^{\prime}$, Phys Rev, B 43, 4641, (1991).

McFeely, F. R., Cartier, E., Terminello, L. J., Santoni, A. and Fischet,ti, M. V., "Soft X-ray-Induced Core Level Photoemission as a Probe of Hot-Electron Dynamics in $\mathrm{SiO}_{2}$ ", Phys, Rev, Lett. 65, 1937. (1990).

McFeely, F. R., Cartier, E., Yarmoff, J. A. and Joyce, S. A., "Low-Energy-Electron Escape Lengths in $\mathrm{SiO}_{2}$ ", Phys. Rev. B 42 , 5191 (1990).

Santoni, A., Himpsel, F. J., "Unoccupied Energy Bands, Exchange Splitting, and Self-Energy of Iron", Phys Rev. B 43, 1305, (1991).

Spiller, E., "Multilayer X-ray Optics", in Optics in Complex Systems, ed. by F. Lanzl, H.J. Preuss, G. Weigelt, ICO XV, 1990; Proc ICO 15 SPIE., Vol. 1319, 687-91, (1990).

Spiller, E., McCorkle, R., Wilczynski, J., Golub, L., Nystrom, G., Takacz, P., Welch, C., "Imaging Performance and Tests of Soft X-ray Telescopes", Proc. SPIE 1343, 134-44, (1990).

\section{Beamline U11}

Grover, J. R., Herron, W. J., Coolbaugh, M. T., Peifer, W. R., Garvey, J. F., "Cluster Beam Analysis via Photoionization", J. Phys. Chem. 95, 6473-6481, (1991).

Nesbitt, F. L., Marston, G., Stief, L. J., Wickramaaratchi, M. A., Tao, W., Klemm, R. B., "Measurement of the Photoionization Spectra and Ionization Thresholds of the $\mathrm{H}_{2} \mathrm{CN}$ and $\mathrm{D}_{2} \mathrm{CN}$ Radicals", J. Phys. Chem. 95, 7613-7617, (1991).

Preses, J. M., Grover, J. R., Kvick, A., White, M. G., "Chemistry with Synchrotron Radiation", American Scientist 78, 424-37, (1990).

\section{Beamline U12B}

McLean, A. B., Ludeke, R., Prietsch, M., Heskett, D., Tang, D., Maeda-Wong, T., "Two-Dimensional Electronic Structure of the GaAs(110)-Bi System", Phys, Rev, B 43, 7243, (1991).

Watson, G. M., Bruhwiler, P. A., Plummer, E. W., Sagner, H.-J., Frank, K.-H., "Two-Dirnensional Band Structure of a Li Layer: Li/Be(0001)", Phys. Rev. Lett. 65, 468, (1990).

\section{Beamline U13UB}

Bjorkholm, J. E., Bokor, J., Eichner, L., Freeman, R. R., Gregus, J., Jewell, T. E., Mansfield, W. M., MacDowell, A. A., Raab, E. L., Silfvast, W. T., Szeto, L. H., Tennant, D. M., Waskiewicz, W. K., White, D. L., Windt, D. L., Wood, II, O. R., Bruning, J. H., "Reduction Imaging at $14 \mathrm{~nm}$ Using Multilayer-Coated Optics: Printing of Features Smaller Than 0.1 $\mu \mathrm{m}$ ", J. Vac. Sci. Tech. B8, 1509, (1990).

Tennant, D. M., Bjorkholm, J. E., O'Malley, M. L., Becker, M. M., Gregus, J. A., Epworth, R. W., "Free-Standing Silicon Microstructures for Soft X-ray Masks and Cold Atom Focusing", J. Vac. Sci. Tech. B8, 1975, (1990).

Tennant, D. M., Raab, E. L., Becker, M. M., O'Malley, M. L., Bjorkholm, J.E., Epworth, R. W., "High Resolution Germanium Zone Plates and Apertures for Soft X-ray Focalometry", J. Vac. Sci. Tech. B8, 1970, (1990).

White, D. L., Bjorkholm, J. E., Bokor, J., Eichner, L., Freeman, R. R., Jewell, T. E., Mansfield, W. M., MacDowell, A. A., Szeto, L. H., Taylor, T. W., Tennant, D. M., Waskiewcz, W. K., Windt, D. L., Wood, II, O. R., "Soft-X-ray Projection Lithography", Solid State Technology 34, 37, (1991).

White, D. L., Bjorkholm, J. E., Bokor, J., Eichner, L., Freeman, R. R., Gregus, J. A., Jewell, T. E., MacDowell, A. A., Mansfield, W. M., Raab, E. L., Silfvast, W. T., Szeto, L. H., Tennant, D. M., Waskiewcz, W. K., Windt, D. L., Wood II, O. R., "Soft X-ray Projection Lithography: Experiments and Practical Printers", Optical and Optoelectronic Applied Science \& Engineering, ed, by R. Hoover, Proc. SPIE 1343 204, (1990). 


\section{Beamline U15}

Hanson, D. M., Ma. C. I., Lee, K, Laplano-Smith, D., Kim, D. Y., "Single-Event, Energy-Resolved, Auger-Electron, MultipleIon Coincidence Mass Spectroscopy", J. Chem. Phys, 93, 9200, (1990).

Krol, A., Resat, H., Sher, C. J., Woronick, S. C., Ng, W., Kao, Y. H., Rehn, V., "Characterization of $\operatorname{In}_{0.53} \mathrm{Ga}_{0.47} \mathrm{As} / \mathrm{InP}$ and $\mathrm{In}_{0,63} \mathrm{Ga}_{0,47} \mathrm{As} / \mathrm{GaAs}$ Epitaxial Structures by Soft X.ray Reflectivity", J. Appl. Phys, 69, 949, (1991).

Krol, A., Sher, C. J., Ming, Z. H., Lin, C. S., Song, L. W., Kao, Y, H., Smith, G. C., Zhu, Y. Z., Shaw, D. T., "Studies of Microstructures in High- $T_{\mathrm{c}}$ Superconductors by X-ray Absorption Techniques", Superconductivity and Applications, ed, by S. H. Kwok, Y.H. Kao, and D. T. Shaw, Plenum Press, NY, (1990).

Lee, K., Kim, D. Y., Ma, C. I., Lapiano-Smith, D. A., Hanson, D. M., "Angular Dependence of Photofragments from K-shell Excited $\mathrm{N}_{2}$ and $\mathrm{O}_{2}$ : Photoabsorption Anisotropy and Excited State Symmetry", J. Chem. Phys., 93, 7936, (1990).

Panessa-Warren, B. J., Tortora, G. T., Stears, R. L., Warren, J. B., "Calcium Imaging With Monochromatic X-rays", Microbeam Analysis, 405-406, (1990).

Woronick, S. C., Ng, W., Krol, A., Kao, Y. H., Arnold, E., "Characterization of $\mathrm{SiO}_{2} / \mathrm{Si}$ Heterostructures by Soft X-ray Reflection", J. Appl. Phys. 69, 1631, (1991).

\section{Beamline U16B}

Peden, C. H. F., Rogers Jr., J. W., Kidd, K. B., Tsang, K.-L., Shinn, N. D., "Synchrotron Photoemission Studies of the Structure of Thermally Grown $\mathrm{Si}_{3} \mathrm{~N}_{4}$ on Si(100)", Mat. Research. Soc. Symp. Proc., Vol. 204, 521, (1991).

Shinn, N. D., Tsang, K.-L., "Strain-Induced Surface Reactivity: Low Temperature Cr/W(110) Nitridation", J. Vac. Sci. Tech. A9, 1558, (1991).

\section{Beamline X1A}

Ade, H., Kirz, J., Hulbert, S., Johnson, E., Anderson, E., Kern, D., "Images of a Microelectronic Device with the X1-SPEM", J. Vac. Sci. Tech., A9, 1902, (1991).

Ade, H., Kirz, J., Hulbert, S., Johnson, E., Anderson, E., Kern, D., "X-ray Spectromicroscopy with a Zone Plate Generated Microprobe", Appl. Phys. Lett., 56, 1841, (1990).

Howells, M. R., Jacobsen, C., Kirz, J., McQuaid, K., Rothman, S. S., "Progress and Prospects in Soft X-ray Holographic Microscopy", Modern Microscopies, ed. by P. J. Duke and A. G. Michette (Plenum, NY) p. 119, (1.990).

Howells, M. R., Jacobsen, C., "X-ray Holography", Synchrotron Radiation News 3, \#4, 23, (1990).

Howells, M., Kirz, J., Sayre, D., "X-ray Microscopes", Scientific American, Feb., 1991.

Jacobsen, C., Howells, M., Kirz, J., Rothman, S., "X-ray Holographic Miscroscopy Using Photoresists", J. Opt. Soc. Am. A7, 1849, (1990).

Kirz, J., "X-ray Microscopy", Synchrotron Radiation News, 4, \#2, 17, (1991).

Rarback, H., et al., "Coherent Radiation for X-ray Imaging - the Soft X-ray Undulator and the X1A Beamline at tha NSLS", J. X-ray Science \& Tech. 2, 274, (1990).

Rothman, S., et al., "Soft X-ray Microscopy in Biology and Medicine: Status and Prospects", Physica Scripta T31, 19, (1990).

Tennant, D. M., Gregus, J. E., Jacobsen, C., Raab, E. L., "Construction and Test of Phase Zone Plates for X-ray Microscopy", Optics Lett. 16, 621, (1991).

\section{Beamline X2B}

Deckman, H. W., Dunsmuir, J. H., D'Amico, K. L., Ferguson, S. R., Flannery, B. P., "Development of Quantitative X-ray Microscopy: Advanced Tomographic Imaging Methods for the Analysis of Materials", Proc. of the Materials Research Society 97, 217, (1991).

D'Amico, K. L., Dunsmuir, J. H., Deckman, H. W., Flannery, B. P., and Roberge, W. G., "The Exxon Microtomography Beamline at the National Synchrotron Light Source", Proceedings of the 4th Int. Conferenc on Synchrotron Radiation Instrumentation, Rev. Sci. Instruments, p. 574, (1992).

Dunsmir, D. H., Ferguson, S. R., and D'Amico, K. L., "Design and Operation of an Imaging X-ray Detector for X-ray Microtomography", Proceedings of the 10th McGee Symp. on Photo-Electronic Imaging Devices, Imperial College, London, (1991).

Dunsmuir, J. H., Ferguson, S. R., D'Amico, K. L., Flannery, B. P., and Deckman, H. W., "X-ray Micortomography and Other Quantitative X-ray Microscopies", Proceedings of the 10th McGee Symp, on Photo-Electronic Imaging Devices, Imperial College, London, (1991). 
Dunsmuir, J. H., Ferguson, S. R., D'Amico; K. L., Flannery, B. P., Deckman, H. W., "X-ray Microtomography: Quantitative Three Dimensional X-ray Microscopy", Rev, of Progress in Quantitative Nondestructive Evaluation 10a, 443, (1991).

Dunsmuir, J. H., Ferguson, S. R., D'Amico, K. L., and Stokes, J, P., "X-ray Micortomography: A New 'Tool for the Characterization of Porous Media", Proceedings of the Annual Meeting of the Society of Petroleum Engineers, 423, (1991).

\section{Beamline X3}

Chu, B., Xu, R., Li, Y. J., Wu, D.·Q., "High Flux X-ray Scattering of Polydiacetylene (P4BCMU) in Dilute Solution", Macromolecules, 22, 3819, (1989).

Chu, B., "Small Angle Xrray Scattering Studies of Sulfonated Polystyrene Ionomers", in Proc, of the 19th Yamada Conf, on Ordering \& Organization in Ionic Solutions, pp. 67-81, (1988).

Chu, B., Wang, J., Shuely, W. J., "Solution Behavior of a Random Copolymer of Poly(isobutyl methacrylate-t-butyl aminoethyl methacrylate): Viscosity and Light Scattering Intensity Studies", Polymer, 31, 805, (1990).

Chu, B., Wu, C., "Structure and Dynamics of Epoxy Polymers", Macromolecules, 21, 1729, (1988).

Chu, B., Wu, D.-Q., MacKnight, W. J., Wu, C., Phillips, J. C., LeGrand, A., Lantman, C. W., Lundberg, R. D., "Synchrotron Small Angle X-ray Scattering of Sulfonated Polystyrene Ionomers", Macromolecules, Communication, 21, 523, (1988).

Chu, B., Wu, C., Li, Y. J., Harbison, G. S., Roche, E. J., Allen, S. R., McNulty, T. F., Phillips, J. C., "Synchrotron X-ray Diffraction of a Single Filament and Bundles of Poly(p-phenylene terephthalamide) Fibers", Polymer Letters, 28, 227, (1990).

Graafsma, H., Majewski, J., Cahen, D., Coppens, P., "Ionic Displacements and Piezoelectric Constants of AgGaS ${ }_{2}$ from X-ray Diffraction of a Crystal in an External Electric Tield", Materials Research Soc. Symp. Proc., Solid State Ionics, Vol, 210, p. 639-643.

Hilfiker, R., Wu, D.-Q., Chu, B., "Synchrotron SAXS Measurements on Solutions of Poly(styrene-isoprene) AB Block Co. polymer in Aniline", J. Coll. \& Interface. Sci., 135, 573, (1990).

Jeffrey, G. A., Piniella, J. F., "The Use of Synchrotron Radiation and its Promise in Charge Density Research, P. Coppens", The Application of Charge Density Research to Chemistry and Drug Design, Series B: Physics, ed. by P. Coppens, G. A. Jeffrey, and J. F. Piniella, (Plenum Press, 1991) Vol. 250, 209.

Lee, P., Graafsma, H., Gao, Y., Sheu, H.-S., Coppens, P., Golden, S. J., Lange, F. F., "Modulated Structure of an $800 \AA \AA$ Epitactic Film of the Superconductor $\mathrm{Bi}_{2} \mathrm{Sr}_{2} \mathrm{CaCu}_{2} \mathrm{O}_{8}$ as Studied by Synchrotron Radiation", Acta Cryst. A47, 67-69, (1991).

Li, Y., Chu, B., "Structure of Aggregates of P4BCMU in Dilute THF/Toluene Solutions", Macromolecules, 24, 4115, (1991).

Paturle, A., Graafsma, H., Sheu, H.-S., Coppens, P., Becker, P., "Measurement of the Piezoelectric Tensor of an Organic Crystal by the X-ray Method: The Nonlinear Optical Crystal 2-methyl 4-Nitroaniline", Phys. Rev. B 43, 683-691, (1991).

Qi, M., Lee, P., Li, R., Coppens, P., Wang, J. H., "Effect of Hydrogen Reduction on the Structure and Conductivity of $\mathrm{YBa}_{2} \mathrm{Cu}_{3} \mathrm{O}_{y}{ }^{\prime \prime}$, Physica $C, 174,23-27,(1991)$.

Schaefer, D. W., Brinker, C. J., Wilcoxon, J. P., Wu, D.-Q., Phillips, J. C., Chu, B., "Precursor Chemistry and the Structure of Silica Aerogels", in Proc, of the Materials Research Soc. Symp., Better Ceramics Through Chemistry, ed. by C. J. Brinker, D. E. Clark and D. R. Urlich, Vol, 121, 691-696, (1988).

Song, H. H., Wu, D.-Q., Chu, B., Satkowski, M., Ree. M., Stein, R. S., Phillips, J. C., "Time-Resolved Small-Angle X-ray Scattering of A High Density Polyethylene/Low Density Polyethylene Blend", Macromolecules, 23, 2380, (1990).

Song, H. H., Stein, R. S., Wu, D.-Q., Ree, M., Phillips, J. C., LeGrand, A., Chu, B., "Time-Resolved SAXS on Crystallization of a LDPE/HDPE Polymer Blend", Macromolecules, 21, 1180, (1988).

Stephens, P., Goldman, A., "The Structure of Quasicrystals", Scientific American, 264, 44-53, (1991).

Stephens, P. W., Mihaly, L., Lee, P. L., Whetten, R. L., Huang, S.-M., Kaner, R. B., Diederich, F., Holozer, K., "Structure of Single-Phase Superconducting $\mathrm{K}_{3} \mathrm{C}_{60}{ }^{\prime \prime}$, Nature, 351, 632, (1991).

van Smaalen, S., de Boer, J. L., Coppens, P., Graatsma, H., "Comment on Charge-Density-Wave Structure in NbSe ${ }_{3}$ Determined by Scanning Tunneling Microscopy", Phys. Rev. Letts., 67, 1471, (1991).

Wang, J., Wang, Z, Peiffer, D., Shuely, W., Chu, B., "Light Scattering and Small Angle X-ray Scattering Studies of an Ionomer Poly(styrene-sodium-2-acrylamido-2-methylpropanesulfonate) in Polar Solvents", Macromolecules, 24, 790, (1991).

Wu, D.-Q., Phillips, J. C., Lundberg, R. D., MacKnight, W. J., Chu, B., "Long Range Inhomogeneity of Sulfonated Polystyrene Ionomers", Macromolecules, 22, 992, (1989).

Wu, D.-Q., Chu, B., "Time-Resolved Small Angle X-ray Scattering and Dynamic Light Scattering Studies of Sol-Gel Transition of Gelatin", in Proc. of the Materials Research Soc, Symp. V, Synch. Rad. in Materials Research, Vol. 143, 203, (1989). 


\section{Beamline X5}

Blanpled, G., Blecher, M., Caracappa, A., Djalali, C., Duval, M. A., Giordano, G., Hoblit, S., Kistner, O. K., Matone, G., Micell, L., Mize, K., Preedom, B. M., Sandorfi, A. M., Schaerf, C., Sealock, R. M., Thorn, C. E., Thorton, S. T., Whisnant, C. S., Babusci, D., Casano, L., D'Angelo, A., Girolami, B., Picozza, P., "Physics with Polarized Photons", Paris 90/7th Int. Conf. on Polarization Phenomena in Nucl. Physics, July 1990.

Hoblit, S., Blanpied, G., Blecher, M., Caracappa, A., Djalali, C., Duval, M.-A., Giordano, G, Kistner, O., Matone, G., Micell, L., Mize, K., Preedom, B., Sandorfi, A., Schaerf, C., Sealock, R., Thorn, C., Thorton, S., Vaziri, K, and Whisnant, C. S., "PhotonBeam Polarization Asymmetries in d( ,p)n", Int. Conf, on Particles and Nuclel, PANIC-XII, I-15, (1990).

Miceli, L., Blanpled, G., Blecher, M., Caracappa, A., Djalali, C., Duval, M.-A., Giordano, G., Hoblit, S., Kistner, O., Matone, G., Mize, K., Preedom, B., Sandorfi, A., Schaerf, C., Sealock, R., Thorn, C., Thorton, S., Vaziri, K., and Whisnant, C. S., "Deuteron Photodisintegration", 76th Int. National Meeting of the Italian Physical Soc., Trento Proceedings, 190, (1990).

Mize, K., Blanpied, G., Blecher, M., Caracappa, A., Djalall, C., Duval, M.-A., Giordano, G., Hoblit, S., Kistner, O., Matone, G., Micell, L., Preedom, B., Sandorfi, A., Schaerf, C., Sealock, R., Thorn, C., Thornton, S., Vaziri, K., Whisnant, C. S., "The Photodisintegration of the Deuteron with Polarized Photons", Bull. Amer. Phys. Soc., 35, No. 4, 928, (1990).

Sandorfi, A., "Beam-Polarization Observables in D(gamma,p)n and the Nuclear Tensor Force, The LEGS Collaboration", Phys. Rev. Lett., 67, 1206, (1991).

\section{Beamline X7A}

Bordet, P., Cox, D. E., Espinosa, G. P., Hodeau, J. L., Marezio, M., "Synchrotron X-ray Powder Diffraction Study of the Phase I' Compound: SnLa $\mathrm{Rh}_{4} \mathrm{Sn}_{12}$ ", Solid State Commun., 78, 359, (1991).

Bringley, J. F., Scott, B. A., La Placa, S. J., Boehme, R. F., Shaw, T, M., McElfresh, M. W., Trail, S. S., Cox, D. E., "The Defect Perovskite Series $\mathrm{LaCuO}_{3-\delta}$, with Copper Valence Varying from $2^{+}$to $3^{+}$", Nature, 347, 263, (1990).

Christensen, A. N., Grand, A., Lehmann, M. S., Cox, D. E., "A Synchrotron X-ray Powder Diffraction Study of the Structure of trans-dichlorotitanium(IV) Tetraphenylporphyrin", Acta Chem. Scand., 44, 103, (1990).

Cox, D.E., "Powder Diffraction", Handbook on Synchrotron Radiation, ed. by G. Brown and D. E. Moncton, (Elsevier Publishers 1991), Vol. 3, (1991).

Crawford, M. K., Farneth, W. E., McCarron, III, E. M., Harlow, R. L., Moudden, A. H., "Oxygen Isotope Effect and Structural Phase Transitions in $\mathrm{La}_{2} \mathrm{CuO}_{4}$-Based Superconductors", Science, 250, 1390, (1990).

Heiney, P. A., Fischer, J. E., McGhie, A. R., Romanow, W. J., Denenstein, A. M., McCauley, Jr., J, P., Smith, III, A. B., Cox, D. E., "Orientational Ordering Transition in Solid C60", Phys. Rev, Lett,, 66, 291.1, (1991).

Hsieh, H. Y., Toby, B. H., Egami, T., He, Y., Poon, S. J., Shiflet, G. J., "Atomic Structure of Amorphous $\mathrm{Al}_{90} \mathrm{Fe}_{\mathbf{x}} \mathrm{Ce}_{10-\mathrm{x}}$ ", J. Material Res., , 2807, (1990).

Kwei, G. H., Von Dreele, R. B., Williams, A., Goldstone, J. A., Lawson, II, A. C., Warburton, W. K., "Structure and Valence from Complementary Anomalous X-ray and Neutron Powder Diffraction", J. Mol. Struct., 223, 383, (1990).

Lightfoot, P., Hriljac, J. A., Pei, S., Zheng, Y., Mitchell, A. W., Richards, D. R., Dabrowski, B., Jorgensen, J. D., Hinks, D. G., "BaBiO ${ }_{2,5}$, A New Bismuth Oxide wtih a Layered Structure", J. Solid State Chem., 473, (1991).

Lightfoot, P., Richards, D. R., Dabrowski, B., Hinks, D. G., Pel, S., Marx, D. T., Mitchell, A. W., Zheng, Y., Jorgensen, J. D., "Phase Separation in $\mathrm{Nd}_{2-\mathrm{x}} \mathrm{Ce}_{\mathrm{x}} \mathrm{CuO}_{4}$, Evidence for Superconductivity at a Single Composition", Physica C, 168, 627, (1990).

Parise, J. B., Wang, Y., Yeganeh-Haeri, A., Cox, D. E., Fei, Y., "Crystal Structure and Thermal Expansion of $\mathrm{Mg}_{0.9} \mathrm{Fe}_{0.1} \mathrm{SiO}_{3}{ }^{\prime \prime}$, Geophys. Research Lett., 17, 2089, (1990).

Rodriguez, M. A., Matheis, D. P., Bayya, S. S., Simmins, J. J., Snyder, R. L., Cox, D. E., "A Search for a Low Temperature Phase Transition Prior to Superconducting Behavior in the $\mathrm{YBa}_{2} \mathrm{Cu}_{3} \mathrm{O}_{7}$ Compound", J. Material Research., 5, 1799, (1990).

Sullivan, J. D., Buttrey, D. J., Cox, D. E., Hriljac, J. A., "A High Resolution Synchrotron X-ray Dif" ،ution Study of Phase Separations in $\mathrm{Pr}_{2} \mathrm{NiO}_{4+\delta}$ ", J. Solid State Chem., 94, 337, (1991).

Vohra, Y. K., Ruoff, A. L., "Static Compression of Metals Mo, Pb, and Pt to 272 GPa: Comparison with Shock Data", Phys. Rev. B 42, 8651, (1990).

Wilkinson, A. P., Cheetham, A. K., Cox, D. E., "Study of Oxidation-State Contrast in Gallium Dichloride by Synchrotron X-ray Anomalous Scattering", Acta Cryst., B47, 155, (1991).

Yan, X., Egami, T., Marinero, E. E., "Bond-Orientational Anisotropy and Anelastic Deformation in Sputter-Deposited Amorphous TbFeCo Films", J. Appl. Phys., 69, 5448, (1991).

Yan, X., Hirscher, M., Egami, T., Marinero, E. E., "Direct Observation of Anelastic Bond-Orientational Anisotropy in Amorphous TbFeCo Thin Films by X-ray Diffraction", Phys. Rev. B43, 9300, (1991). 
Zemva, B., Lutar, K., Jesih, A., Casteel, Jr, W. J., Wilkinson, A. P., Cox, D. E., Von Dreele, R. B, Borrmann, H, and Bartlett, N. J., Am. Chem. Soc, 113, 4192, (1991).

Zolliker, P., Cox, D, E., Parise, J. B., McCarron, III, E, M., Farneth, W. E., "A Neutron and Synchrotron X.ray Powder Dif. fraction Study of $\mathrm{La}_{2} \mathrm{CuO}_{4+\delta^{\prime \prime}}$, Phys. Rev, B 42, 6332, (1990).

\section{Beamline X7B}

Artioli, G., Kvick, A., "Synchrotron X-ray Rietveld Sturly of Perlialite, the Natural Counterpart of Synthetic Zeolite-L", Eur. J. Mineral, 2, 749-759, (1990).

Berman, A., Addadi, L., Kvick, A., Leiserowitz, L,, Nelson, M., and Weiner, S., "Intercalation of Sea Urchin Proteins in Calcite: Study of a Crystalline Compostle Material", Science, 250, 664-667, (1990).

Chen, J, M., Yang, X. Q., Chapman, D., Nelson, M., Skotheim, T. A., Ehrlich, S. N., Rosner, R. B., Rubner, M. F," "Temperature Dependent Structure of Conducting Langmuir Blodgett Films Studied by X-ray Scattering", Molec, Cryst. Liq. Cryst., 191, 145-153, (1990).

Preses, J. M., Grover, J. R., Kvick, A., White, M. G., "Chemistry with Synchrotron Radiation", Amer, Sci., 78, 424-437, (1990).

Zhao, W., Zhao, X., Rafailovich, M. H., Sokolov, J., Mansfield, T., Stoin, R. S., Composto, R. C., Kramer, E. J., Jones, R. A. L., Sansone, M., Nelson, M., "Neutron and X-ray Reflectivity Measurements of Polystyrene/Polybromostyrene (PS/PBrS) Interfaces", Physica B., 173, 43-46, (1991).

\section{Beamline X9A}

Bowers, C., Synthesis and Spectroscopy of Zeolite Encapsulated Metal Complexes, Doctoral Thesis, Ohio Stato University, (1990).

Carmeli, C., Lewis, A., and Jagendorf, A. T., "EXAFS Analysis of the Structure of Mn Nucleotide Bound to Latent and Activated CF1-ATP-ase", Photosynthesis Res., 685-690.

Colpas, G. J., Maroney, M. J., Bagyinka, C., Kumar, M., Willis, W. S., Suib, S. L., Mascharak, P. K., "X-ray Spectroscopic Studies of Nickel Complexes, with Application to the Structure of Ni Sites in Hydrogenases", Inorg. Chem., 30, 920-928, (1991).

DeRose, V. L., Structural Studies of Manganese and Halide on the Photosystem II Oxygen Evolving Complex, Doctoral Thesis, University of California at Berkeley, (1990).

DeRose, V. J., Latimer, M. J., Mukerji, I., Yachandra, V. K., Sauer, K., and Klein, M. P., "Cofactor Effect on the Oxygen Evolving Manganese Center Determined by X-ray Absorption Spectroscopy", Third International Conference on Blophysics and Synchrotron Radiation, July 1990, p. 129.

Furt,ak, T, E., Wang, L., Creek, E. A., Samanta, P., Hayes, T, M., Kendall, G., Li, W., Liang, G., and Lo, C. M., "Surface Ex. tended X-ray Absorption Fine Structure of Copper on a Platinum Electrode", Interdisciplinary Conf, on Electrified Interfaces, Asilomar, CA, September 16-21, 1990.

Idziak, S. H. J., Maliszewskyj, N. C., Heiney, J, P., McCauley, P. A., Jr., Sprengler, P. A., and Smith, III, A. B., "Structure and Mesophases of Hexacyclene Derivatives", J. Am. Chem. Soc., 113, 7666-7672, (1991).

Maroney, M. J., Colpas, G. J., and Bagyinka, C., "XAS Studies of Ni Model Compounds Wtth Application to the Structure of Ni Sites in Thiocapsa roseopersicina Hydrogenase", Proceedings of the Third International Conference on Biophysics and Synchrotron Radiation, 141-143 (1990).

Maroney, M. J., Colpas, G. J., Bagyinka, C., Baidya, N., and Mascharak, P, K., "EXAFS Investigations of the Ni Site in Thiocapsa rosoepersicina Hydrogenase: Evidence for a Novel Ni, Fe, S, Cluster", J. Am. Chem. Soc., 113, 3962-3972, (1991).

Maroney, M. J., Colpas, G. J., and Bagyinka, C., "XAS Structural Investigations of the Ni Site in Reduced Thiocapsa roseopersicina Hydrogenase", J. Am. Chem. Soc., 112, 7067-7068, (1990),

Penner-Hahn, J. E., Fronko, R. M., Waldo, G. S., Yocum, C. F., Bowlby, N. R., Betta, S. D., "X-ray Absorption Spectroscopy of the Photosynthetic Oxygen", Current Res, in Photosyn., ed, by N. Baltchessky, 1, 797-800, (1990).

Pettingill, T. M., Strange, R. W., Blackburn, N. J., "Carbonmonoxy Dopamine- $\beta$-hydroxylase", J. Biol. Chem., 266, 1699617003, (1991).

Sagi, I., Wirt, M. D., Chen, E., Frisbie, S., Chance, M. R., "Structure of an Intermediate of Coenzyme B 12 Catalysis by EXAFS: Cobalt(II) B 12 " J. Amer. Chem. Soc,, 112, \#24, (1990).

Sagi, I., Wirt, M., Chen, E., Frisbie, S., Lee, R., Chance, M., "Structure of an Intermediate of Coenzyme B 12 Catalysis by EXAFS: Co(II) B B2", J. Am. Chem. Soc., 112, 8639-8644, (1990). 
Sanyal, I, Strange, R, W., Blackburn, N, J, Karlin, K, D., "Formation of a Copper-Dioxygen Complex $\left(\mathrm{Cu}_{2} \cdot \mathrm{O}_{2}\right)$ Using a Simple Imidazole Ligands", J, Am. Chem. Soc., 113, 4692-93, (1981).

Sinclair, R., Bumpus, J., Chang, C.-S., Yamazakl, I, Powers, L." "The Structure of the Active Site of the Heme Peroxidasesi A Comparative Study", Proceedings from the 10th International Blophysics Congress, 93, (1990).

True, A, E., Orville, A, M., Pearce, L, L, Lipscomb, J, D., Que, Jr, L, "An EXAFS Study of the Interaction of Substrate with the Ferric Active Site of Protocatechuate 3,4-Dioxygenase", Bioohemistry, 29, 10847-10854, (1990).

Twu, J., Synthetic, Mechanistic and Structural Chemistry of Zeolitic and Intercalation Materiale, Doctoral 'Thesis, Ohio State University, (1990).

Whitehead, J, P., Colpas, C, J, Bagyinka, C., Maroney, M. J, "An X-ray Absorption Spectroscopic Study of the Reductive Activation of Thiocapsa roseopersicina Hydrogenaso", J. Am. Chem. Soc., 113, 6288-6289, (1991).

Wirt, M., Sagi, I., Chen, E., Frisbie, S. Lee, R., Chance, M., "Geometric Conformations of Intermediates of $B_{12}$ Catalysis by X.ray Edge Spectroscopy: Co(I) $\mathrm{B}_{12}, \mathrm{Co}(11) \mathrm{B}_{12}$, and Base-Off Adenosycobalamin", J. Am. Chem. Soc., 113, 5299, (1991).

Zhang, K., Reddy, K. S., Bunker, G. B., Chance, B., "Active Site Conformation in Myoglobin as Determined by X.ray Absorption Spectroscopy", Proteins: Structure, Function, and Genetics, 10, 279-286, (1991).

Zhang, K., Chance, B., Reddy, K. S., Ayene, I., Stern, E, A., Bunker, G. "Structural Differences in Solution and Crystalline Forms of Met-Myoglobin", Biochemistry, 30, 9116-9120, (1991).

\section{Beamline X10A/B}

Chang, S. L., King, Jr, H. E., Huang, M.'T., Gao, Y., "Direct Phase Determination of Large Macromolecular Crystals Using Three-Beam-X-ray Interference", Phys. Rev, Lett., 67, 3111, (1991).

Eisenberger, P., Fenter, P., and Liang, K. S., 2nd Surface X-ray and Neutron Diffraction, ed, by H, Zabel, and I, K. Robinson (Springer-Verlag).

Fenter, P., Jun, L., Camillone, III, N., Bernasek, S., Elsenberger, P., Liang, K, Ramanarayanan, T. A., Scoles, G., "The Structure of $\mathrm{CH}_{3}\left(\mathrm{CH}_{2}\right)_{17} \mathrm{SH} / \mathrm{Ag}(111)$ : An Incommensurate Self-Assembled Monolayer", Langmuir, 7, 2013, (1991).

Hung, H. H., Liang, K. S., Lee, C. H., Lu, 'T, M., "Microstructure of Expitaxial Al(111)/Si(111) Films", in Evolution of Thin Films and Surface Microstructure, MRS Sym. Proc. Vol, 202, (1991), p. 301.

King, Jr, H. E., Mundl, L. A, Strohmaier, K. G., and Haushalter, R. C., "A Synchrotron Single Crystal X-ray Structure Determination of a Small Crystal: Mo-Mo Double Bonds in the 3-D Microporous Molydbenum Phosphate $\mathrm{NH}_{4}\left[\mathrm{Mo}_{2} \mathrm{P}_{2} \mathrm{O}_{10}\right] \mathrm{H}_{2} \mathrm{O}^{\prime \prime}$, J. Solid State Chem., 92, 1, (1991).

King, Jr., H. E., Mundi, L. A., Strohmaier, K. G., and Haushalter, R. C., "A Synchrotron Single Crystal X-ray Structure Determination of $\left(\mathrm{NH}_{4}\right)_{3} \mathrm{Mo}_{4} \mathrm{P}_{3} 0_{16}$ : A Microporous Molybdenum Phosphate with $\mathrm{Mo}_{4} 0_{4}{ }^{++}$Cubes", J. Solid State Chem., 92, 154, (1991).

Lee, C. H., Liang, K. S., Sheu, F. S., Sass, S. L., Flynn, C. P., "Interface Structure of Epitaxial Nb Films on Sapphire", in Defects in Materials, MRS Sym. Proc. Vol. 209, (1991), p. 679.

Liang, K, S., Chien, F. Z., Hughes, C. J., Meitzner, G. D., Sinfelt, J, H., "The Nature of Rhenium in Silica-Supported Pt-Re Clusters: Synchrotron X-ray Diffraction Studies", The J. of Phys, Chem., 95, (1990).

Liang, K. S., D-Amico, K, L., Lee, C, H, and Sheu, E. Y., "Domain-Wall Pinning in Uniaxial Phases of Pb Adlayers on a Cu(110) Surface", Phys. Rev. Lett., 65, 3032, (1990).

Liang, K. S., Hung, H. H., Chang, S. L., Fu, Z, Moss, S, C., and Oshima, K., "Surface-Induced Order Observed on a $\mathrm{Cu}_{3} \mathrm{Au}(001)$ Surface", in Surface X-ray and Neutron Scattering, ed. by H. Zabel and I. K. Robinson (Springer-Verlag).

Rieker, 'T, P., Clark, N. A., Safinya, C. R., "Chevron Layer Structures in Surface Stabilized Ferroelectric Liquid Crystal (SSFLC) Cells Filled with a Material Which Exhibits the Chiral Nematic to Smectic-C* Phase Transition", Ferroelectrics, 113, $245,(1991)$.

Rivers, S. B., Unertl, W. N., Hung, H. H., Liang, K. S., "Kinetics of Surface Induced Ordering on Cu, $\mathrm{Cu}(001)$ Surface", in Kinetics of Phase Transformation, MRS Sym. Proc. Vol, 205, (1991).

Safinya, C. R., Sirota, E. B., and Plano, R., "The Nematic to Smectic-A Phase Transition Under Shear Flow: A NonEquilibrium Synchrotron X-ray Study", Phys. Rev. Lett., 66, 1986, (1991).

Safinya, C. R, Sirota, E. B., Plano, R., and Bruinsma, R. F., "A Nonequilibrium Synchrotron X-ray Study of a Liquid Crystal Under Shear Flow", J, Phys. Condes, Matter, 2, SA365, (1990).

Safinya, C. R., Sirota, E. B., Plano, R, and Lei, N., "Non-Equilibrium X-ray Study of the Nematic-Smectic-A Transition Under Shear Flow", Macromolecular Liquids, Materials Research Society Publishers, ed, by E.R. Safinya, S.A. Safran, and P.A.

Pinkus, Publishers, 177, (1990), p. 165. 
Satija, S, K., Majkrzak, C, F, Russell, T', P., Sinha, S, K, Sirota, E, B., Hughes, G, J." "A Neutron Reflectivlty Study of Block Copolymers Adsorbed from Solution", Macromoleoules, 23, 3860, (1990).

Shen, Y., Safinya, C. R., Fetters, L, Adam, M., Witten, T, and Hadjichristidis, N., "Macrolattice Formation in Amorphous Associating Polymers", Phys, Rev, A, 43, 1886, (1981).

Shen, Y., Safinya, C. R., Fetters, L, Adam, M., Witten, T., Llang, K. S., Chance, R., Stokes, J., "X.ray Diffraction Studies of Zwitterionic Assoclating Polymers", in Macromolecular Liquids, Materials Research Soclety Publlshers, ed, by E.R. Safinya, S.A. Safran, and P.A. Pinkus, 177, (1990), p. 27.

Sirota, E. B., Hughes, G. J., Sinha, S, K., Satija, S. K., and Russell, T, P., "Ray Reflectivity and Diffuse Scattering from Ordered Polymer Films", Polymer Preprints, 31, 81, (1990).

Sirota, E. B., Safinya, C. R., Smith, G, S, Plano, R., Roux, D., and Clark, N. A., "Universal Behavior in Phospholipid Multimembrane Systems", Geometry and Thermodynamics, ed. by J.C. Toledano, (Plenum, New York, 1990).

Smith, G. S., Sirota, E. B., Safinya, C. R, and Clark, N. A., "X-ray Structural Studies of Treely Suspended Hydrated DMPC Multimembrane Films", J. Chem. Phys, 92, 4519, (1990).

\section{Beamline X11 A \& B}

Barerra, E. V., Maruyaina, B., Heald, S. M., "Fracture EXAFS: A New Method for the Study of Interfaces in Thin Films and Pollycrystalline Materials", Proc. MRS 188, 275, (1990).

Fung, A. S., Tolley, P. A., Koningsberger, D. C., Kelley, and M. J., Gates, B. C., "Cationic Trirhenium Rafts on y-Alumina: Characterization by X-ray Absorption Spectroscopy", J. of Phys. Chem., 95, 225, (1991).

Heald, S. M., and Barrera, E. V., "Glancing Angle X-ray Study of the Effect of Oxygen on Interface Reactions in Al/Ni Bilayers", J. Material Research., 6, 935, (1991).

Kirlin, P. S., van Zon, F. B. M., Koningsberger, D. C., and Gates, B. C., "Surface Catalytic Sites Prepared from $\left[\mathrm{HRe}(\mathrm{CO})_{6}\right]$ and $\left[\mathrm{H}_{3} \mathrm{Re}_{3}(\mathrm{CO})_{12}\right]$ : Mononuclear, Trinuclear, and Metalle Rhenium Catalyst Supported on MgO", J, of Phys. Chem., 94, 8439, (1990).

Maloney, S. D., van Zon, F. B. M., Kelley, M. J., Koningsberger, D. C., and Gates, B. C., "A Well-defined Supported Metal Catalyst: $\operatorname{Ir}_{4} / \mathrm{MgO}^{\prime \prime}$, Catalysis Letters, 5, 161, (1990).

Maloney, S. D., Zhou, P.-L., Kelley, M. J., and Gates, B. C., "Osmium Carbonyl Clusters in Basic Zeloite Y: Characterization by Extended X-ray Absorption Fine Structure Spectroscopy", J. of Phys. Chem., 95, 5409, (1991).

Maruyama, B., Henshaw, W. M., Barerra, E. V., Everett, R, K., Heald, S. M., "Extended X-ray Absorption Fine Structure Analysis Applied to Composite Materials", in Controlled Interfaces in Composite Materials, ed. by H. Ishida, 175, (1990).

Rabenburg, L., Barrera, E. V., Maury, C. E., Allibert, C. H., Heald, S. M., "Extended X-ray-absorption Fine-Structure Study of the Position of $\mathrm{Zr}$ within the Unit Cell of $\mathrm{Sm}_{2} \mathrm{Co}_{17}{ }^{\prime \prime}, J$. Appl. Phys., 69, 5568, (1991).

Tan, Z., Budnick, J. I., Chen, W. Q., Brewe, D. L., Cheong, S.-W., Cooper, A. S., Rupp, R. W., "Observation of Ianthanum and Rare-earth-site ordering in T*-phase $\mathrm{La}_{2-\mathrm{x}-\mathrm{y}} \mathrm{R}_{\mathrm{x}} \mathrm{Sr}_{y} \mathrm{CuO}_{4}(\mathrm{R}=\mathrm{Sm}, \mathrm{Eu}, \mathrm{Gd}, \mathrm{Tb})$ ", Phys, Rev. B 42, 4808, (1990).

Tan, Z., Budnick, J. I., Chen, W. Q., Weinberger, B, R., Lynds, L., "X-ray Absorption Studies of Vanadium-containing $\mathrm{YBa}_{2} \mathrm{Cu}_{3} \mathrm{O}_{7}{ }^{\prime \prime}$, Mater. Res. Soc. Symp. Proc. 169, 229, (1990).

Tan, Z., Budnick, J. I., Luo, S., Chen, W. Q., Cheong, S.-W., Cooper, A. S., Canfield, P. C., Fick, Z., "X-ray-absorption Studies of Cation Ordering and Valence in the T*-phase $\mathrm{La}_{2-x-y} \mathrm{R}_{\mathrm{x}} \mathrm{Sr}_{y} \mathrm{CuO}_{4}(\mathrm{R}=\mathrm{Sm}, \mathrm{Eu}, \mathrm{Gd}$, Tb and Dy)", Phys. Rev, B 44, 7008, (1991).

Tan, Z., Budnick, J. I., Pease, D. M., Namavar, $F_{.,}$"X-ray-absorption Studies of Krypton Precipitates in Solid Matrices", Phys, Rev. B 43, 1987, (1991).

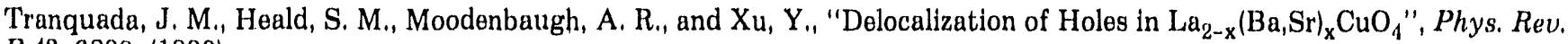
$B$ 42, 6299, (1990).

Zhou, W., Paesler, M. A., Sayers, D. E., "Structure of Germanium-Selenium Glasses: An X-ray Absorption and Structure Study", Phys, Rev. B 43, 2315, (1991).

\section{Beamline X12B}

James, V. J., McConnell, J. F, Capel, M. S., "The d-spacing of Collagen from Mitral Heart Valves Changes with Aging", $B B A$ 1078: 1, pp. 19-22, (1991).

Sheu, E., Chen, S. H., Carvalho, B. L., Lin, J. S., Capel, M. S., "Interpretation of Small-Angle X-ray Scattering Intensity Distributions from Fluctuating Lamellae in a Ternary System: Sodium Octyl Sulfate-water-decanol.", Langmuir, 7:9, pp. 1895-9, (1991). 


\section{Beamline X12C}

Lee, Kim, Sweet, and Suh, "Crystallization and a Preliminary X-ray Crystailographic Study of $\alpha$-amylase from B. Licheniformis", Archres Biochem \& Biophys, 291:20, (1991).

Mottener, Strand, Symesky, Sweet, Dawley, Geoghagan, Gerard, and Goldsmith, "Structural Basis of Latency in Plasminogen Activator Inhlbitor-I", Nature, (1991).

\section{Beamline X14}

Chen, L, C., Spaepen, F., Robertson, J, L., Moss, S, C., and Hiraga, K., "A Structural and Calorimetric Study of the Transformation in Sputtered Al-Mn and Al-Mn-S1 Films", J, Material Res, 5, 1871, (1990).

Cheng, S. Z. D., Zhang, A., Barley, J. S., Chen, J., Habenschuss, A., Zschack, P., "Isothermal Thickening and Thinning Processes in Low Molecular Weight Poly(ethylene oxide) Fractions, 1. From Nonintegral Folding to Integral Folding Chain Crystal Transitions", Macromolecules, 24, 3937, (1991).

Dutta, P., "Phase Transitions in Lipid Monolayers on Water: New Light on an Old Problem", in Phase Transitions in Surface Films 2, ed. by H. Taub, G. Torzo, H. J. Lauter, and S. C. Faine. Plenum Publishing Company, N.Y., (1991), p. 183.

Isaacs, E. D., McWhan, D. B., Kleinman, R. N., Bishop, D. J., Ice, G. E., Zschack, P., Gaulin, B. D., Mason, T. E., Garrett, J. D., Buyers, W. L. J., "X-ray Magnetic Scattering in Antiferromagnetic URu ${ }_{2} \mathrm{Si}_{2}$ ", Phys. Rev. Lett., 65, 3185, (1990).

Kan, $\mathrm{X}$., The Incommensurate Modulation and Attendant Defects in $\mathrm{Bi}_{2} \mathrm{Sr}_{2} \mathrm{CaCu}_{2} \mathrm{O}_{x}$ Single Crystals, Ph.D. Dissertation, Department of Physics, Universtiy of Houston, Houston, TX, October, 1990.

Kan, X. B., Kulik, J., Chow, P. C., Moss, S. C., Yan, Y. F., Wang, J. H., and Zhao, Z. X," "X-ray und Electron Diffraction Study of Single Crystal $\mathrm{Bi}_{2} \mathrm{Sr}_{2} \mathrm{CaCu}_{2} \mathrm{O}_{\mathbf{x}}{ }^{\prime \prime}$, J. Mater, Res., 5, 731, (1990).

Kumar, R., Sparks, C. J., Shiraishi, T., Specht, E. D., Zschack, P., Ice, G. E., Hisatsune, K., "X-ray Determination of Site Occupation Parameters in Ordered Ternaries $\mathrm{Cu}\left(\mathrm{Au}_{\mathrm{x}} \mathrm{M}_{1-\mathrm{x}}\right) \mathrm{M}=\mathrm{Ni}$, Pd", Material Res. Soc. Symp. Proc. 23, 369, (1991).

Lin, B., X.ray Diffraction Studies of Fatty Acid and Alcohol Monolayers on the Surface of Water, Ph.D. Dissertation, Department of Physics and Astronomy, Northwestern University, Evanston, IL, December, (1989).

Lin, B., Bohanon, T., Shih, M., and Dutta, P. "X-ray Diffraction Studies of the Effects of Calcium and Copper Ions on Langmuir Monolayers of Heneicosanoic Acid", Langmuir, 6, 1665, (1990).

Lin, B., Shih, M. C., Bohanon, T., Ice, G. E., and Dutta, P., "The Phase Diagram of a Lipid Monolayer on the Surface of Water", Phys. Rev. Lett. 65, 191, (1990),

McWhan, D. B., Vettier, C., Isaacs, E. D., Ice, G. E., Siddons, D. P., Hastings, J. B., Peters, C., and Vogt, O., "Magnetic Scattering Study of Uranium Arsenide", Phys, Rev, B 42, 6007, (1990).

Rao, S. I., Houska, C. R., Grabowski, K., Ice, G, E., and Sparks, C. J., "X-ray Diffuse Scattering from a Nitrogen-Implanted Niobium Film", J. Appl. Phys, 69, 8104, (1991).

Rebonato, R., Ice, G. E., Habenschuss, A., and Bilello, J. C., "High Resolution Microdiffraction Study of Notch-Tip Deformation in Mo Single Crystals Using X-ray Synchrotron Radiation", Philos. Mag,, A60, 571, (1989).

Robertson, J. L., Random-Cluster Models for Icosahedral Phase Alloys, Ph.D. Dissertation. Department of Physics, University of Houston, Houston, TX, December, (1990).

Robertson, J. L., Moss, S. C., "Atomic Decoration of a Random Cluster Model For Icosahedral Phase AlMnSi", Phys. Rev. Lett. 66, 353, (1991).

Robertson, J. L., Moss, S. C., "Random-Cluster Models for Icosahedral Phase Alloys", J. Phys. B. - Cond. Matt. 83, 391, (1991).

Robertson, J. L., Moss, S. C., and Kreider, K. G., "Comparison of Amorphous and Quasicrystalline Films of Sputtered $\mathrm{Al}_{0.72} \mathrm{Mn}_{0,22} \mathrm{Si}_{0,06}$ ", Phys. Rev. Lett. 60, 2062, (1988).

Sparks, C. J., Specht, E. D., Ice, G. E., Zschack, P., and Schneibel, J., "Bonding Energies and Long-Range Order in the TriAluminides", Mat. Res. Soc. Symp. Proc. 213, 363, (1991).

Specht, I. D., Ice, G. E., Peters, C. J., Sparks, C. J., Lukas, N., Zhu, X. M., Moret, R., and Morkoc, H., "Characterization of GaAs/Si Interface Structure by X-ray Diffraction", Mat. Res, Soc. Symp. Proc, 208, 321, (1991).

Specht, E. D., Ico, G. E., Peters, C. J., Sparks, C. J., Lukas, N., Zhu, X. M., Moret, R., and Morkoc, H., "X-ray Diffraction Measurement of Interface Structure in GaAs/Si (001)", Phys, Rev, B, 43, (1990). 


\section{Beamline X15A}

Denlinger, J. D., Olmstead, M. A., Rotenberg, E., Fontes, E., and Patel, J. R., "Atomic-size Effects on the Growth of SrF 2 and $(\mathrm{Ca}, \mathrm{Sr}) \mathrm{F}_{2}$ on Si(111)", Phys. Rev. 43, 7335, (1991).

Vlieg, E., Fontes, E., and Patel, J. R., "Structure Analysis of Si(111)-( $\sqrt{3} \times \sqrt{3})$ R30/Ag Using X-ray Standing Waves", Phys. Rev. $B$ 43, 7185, (1991).

\section{Beamline X16A}

Ferrer, S., Vlieg, E., and Robinson, I. K., "Epitaxial Submonolayer Cobalt Films on Cu(100) Studied by X-ray Diffraction", Su, face Science, 250, L363, (1991).

Robinson, I. K., "Discussion of the Crystal Truncation Rod Formalism", Faraday Discussions of the Royal Soc. of Chem., 89, 208, (1990).

Robinson, I. K., "Roughening or Ising Transition of Pt(110)", Faraday Discussions of the Royal Soc. of Chem., 89, 201, (1990).

Robinson, I. K., Vlieg, E., and Kern, K., "Roughening or Ising Transition of Pt(110): Reply to Comment of J. Villain and I. Vilfan", Phys. Rev. Lett. 65, 1831, (1990).

Robinson, I. K., Vlieg, E., Kern, K., "Structure and Roughening of the Pt(110) Surface", Faraday Discussions of the Royal Soc. of Chem., 89, 159, (1990).

Robinson, I. K., "Surface Structural Techniques Applied to Interfaces", Materials Res. Soc. Bulletin, September (1990), p. 38.

Zhu, X-M., Moret, R., Zabel, H., Robinson, I. K., Vlieg, E., and Fleming, R. M., "Grazing Incidence X-ray Study of the Charge Density Wave Phase Transition in $\mathrm{K}_{0.3} \mathrm{MoO}_{3}{ }^{\prime \prime}$, Phys. Rev. B 42, 8791, (1990).

Zhu, X-M., Zabel, H., Robinson, I. K., Vlieg, E., Dura, J. A., Flynn, C. P., "Surface Induced Heterophase Fluctuation", Phys. Rev. Lett., 65, 2692, (1990).

\section{Beamline X17B1}

Wang, Y., Weidner, D., Liebermann, R. Liu, X., Ko, J., Vaughan, M., Zhao, Y., Yeganeh-Haeri, A., and Pacalo, R., "Phase Transition a ad 'Thermal Expansion of $\mathrm{MgSiO}_{3}$ Perovskite", Science, Vol. 251, (1991).

\section{Beamline X17B2}

Thomlinson, W., Gmür, N., Chapman, D., Garrett, R., Lazarz, N., Morrison, J., Reiser, P., Padmanabhan, V., Ong, L., Green, S., Thompson, A., Zeman, H., Hufstadter, R., Brown, G., Giacomini, J., Gordon, H., and Rubenstein, E., "Venous Synchrotron Coronary Angiography", The Lancet, 337, 360, (1991).

Zeman, H. D., DiBianca, F. A., Thomlinson, W. C., "A Kinestatic Charge Detector for Intravenous Coronary Angiography Using Synchrotron Radiation X-rays", IEEE Trans. on Nucl. Science, 38, 641, (1991).

\section{Beamline X18A}

Cohen, J. B., "X-ray Diffraction Studies of Catalysts", Ultramicroscopy, 34, 41-46, (1990).

Hoffman, S. A., Venkatraman, C., Ehrlich, S. N., Durbin, S. M., and I iedl, G. L., "Structural and Transport Measurements in $\mathrm{La}_{(1.8)} \mathrm{Sr}_{(0.2)} \mathrm{NiO}_{(4+\mathrm{x})}{ }^{\prime}$, Phys. Rev. B 43, 10, 7852-58, (1991).

Levine, J. R., Cohen, J. B., and Chung, Y. W., "Thin Film Island Growth Kinetics: A Grazing Incidence Small Angle Scattering Study of Gold on Glass", Surface Science, 248, 215-224, (1991).

Mahalingham, K., Mahadev, V., Liedl, G. L., Samders, Jr., T. H., "Precipitation Behavior of Delta-prime in a Binary Al-Li Alloy", Script Met, 25, 2181-86, (1991).

Quintana, J. P., "Practical Equation for Deadtime Determination in X-ray Counting Systems", J. Appl. Cryst., 24, 261-262, (1991).

Quintana, J. P., Butler, B. D., and Haeffner, D. R., "Experimentally Determined Anomalous Scattering Factors for Mn, Fe, $\mathrm{Cu}, \mathrm{Zn}$, and Hg Using the Kramers-Kronig Relation", J. Appl Cryst., 24, 184-187, (1991).

\section{Beamline X18B}

Alp, E. E., Mini, S., Ramanathan, M., “X-ray Absorption Spectroscopy: EXAFS \& XANES”, Proceedings of Synchrotron $X$-ray Sources and New Opportunities in the Agricultural and Related Science Workshop, Jan. 8-10, 1990, Argonne, ANL/ APS/TM-7 (1990). 
Alp, E. E., Mini, S., Ramanathan, M., Goodman, G. L., "Probing Charge on Cu in Oxide Superconductors", Superconductivity \& Applications, ed. by H.S. Kwok, Plenum Press, New York, (1990), p. 313.

Alp, E. E., Mini, S., Ramanathan, M., Hyun, O. B., "X-ray Absorption Spectroscopy with Polarized Synchrotron Radiation", SPIE 1345, 137, (1990).

Antonio, M. R., Song, I., and Yamada, H., "Coordination and Valence of Niobium in $\mathrm{TiO}_{2}-\mathrm{NbO}_{2}$ Solid Solutions Through X-ray Absorption Spectroscopy", J. of Solid State Chem. 93, 183, (1991).

Lince, J. R., Hilton, M. R., and Bommannavar, A. S., "Oxygen Substitution in Sputter-Deposited $\mathrm{MoS}_{2}$ Films Studied by Extended X-ray Absorption Fine Structure, X-ray Photoelectron Spectroscopy, and X-ray Diffraction", Surface and Coatings Tech. 43/44, 640, (1990).

Mini, S. M., Alp, E. E., Ramanathan, M., Bommannavar, A., Hyun, O. B., "Polarization Dependent EXAFS Studies in Layered Copper Oxide Superconductors", SPIE 1345, 260, (1990).

Salem Sugui, Jr., S., Alp, E. E., Mini, S. M., Ramanathan, M., Campuzano, J. C., Jennings, G., Faiz, M., Pei, S., Dabrowski, B., Zheng, Y., Richards, D. R., Hinks, D. G., "Determination of Local Structure in Bal-xKxBiO3 by X-ray Absorption Spectroscopy", Phys. Rev. B 43, (1991).

\section{Beamline X19A}

Davenport, A. J., Isaacs, H. S., Frankel, G. S., Schrott, A. G., Jahnes, C. V., Russak, M. A., J. of Electrochem. Soc., Vol. 138, (1991), pp. 337.

Huffman, G. P., Huggins, F. E., Francis, H. E., Mitra, S., and Shah, N., "Structural Characterization of Sulfur in Bioprocessed Coal", Coal Sci. and Tech., Vol. 16: Processing \& Utilization of High-Sulfur Coals III, (3rd International Conference, Ames, IA), ed. by R. Markuszewski and T.D. Wheelock, 21-32, (1990).

Huffman, G. P., Huggins, F. E., and Shah, N., "Behavior of Basic Elements During Coal Combustion", Prog. Energy Combust. Sci. 16, 243-251, (1990).

Huffman, G. P., Huggins, F. E., and Shah, N., "XAFS Spectroscopy: A New Technique for Investigating Impurity Elements in Coal that Control Slagging and Fouling Behavior", Conference, Santa Barbara, CA, ed. by R. W. Bryers and K. S. Vorres, pp. 63-73, Engineering Foundation, New York, NY (1990).

Srinivasachar, S., Helble, J. J., Boni, A. A., Shah, N., Huffman, G. P., Huggins, F. E., "Mineral Behavior During Coal Combustion. 2. Illite Transformations", Prog. Energy Combust. Sci., 16, 293-302, (1990).

\section{Beamline X19C}

Chiang, F. P., Li, X. M., Wu, J., and Dudley, M., "Expe rimental Measurement of Crack. Tip Strain Field in a Single Crystal", Proc. of Mecamat '91, International Seminar on Large Plastic Deformations, Fontainbleau, France, (1991).

Dudley, M., "The Application of Neutron Topography to the Study of X-ray Sensitive Organic Single Crystals - A Possible Alternative to X-ray Topography", in Neutron Scattering for Materials Science, ed. by S. Shapiro, S. C. Moss, and J.D. Jorgenson, Mat. Res. Soc. Symp. Proc., 166, 55-60, (1990).

Dudley, M., Baruchel, J., and Sherwood, J. N., "Neutron Topography as a Tool for Studying Reactive Organic Crystals: A Feasibility Study", J. Appl. Cryst., 23, 186-98, (1990).

Dudley, M., DiSalvo, R., Foxman, B. M., and Jones, $\mathrm{W}^{\prime}$., "Characterization of Defects in p-terpheny' Single Crystals", Mol. Cryst. Liq. Cryst., (1991).

Dudley, M., DiSalvo, R., Gordon-Smith, D., and Jones, W., "The Role of Defects in a Low Temperature Phase Transition in an Organic Single Crystal", Mol. Cryst. Liq. Cryst., (1991).

Dudley, M., Hou, S. Y., and Foxman., B. M., "Synchrotron White Radiation Topographic Studies of The X-ray Induced Solid State Polymerization of Bis (Propiolato) TetraAquoZinc(II) Single Crystals", Mol. Cryst. Liq. Cryst., 187, 207-13, (1990).

Dudley, M., Sherwood, J. N., and Bloor, D., "X-ray Topographic Studies of Solid State Reaction", 2. "Solid State Polymerization of 2,4-HexadiyneDiol-Bis-(p-Tolene Sulphonate) [PTS]", Proc. R. Soc. Lond., A434, 243-61, (1991).

Dudley, M., Tolis, G., Gordon-Smith, D., and Fazi, C., "Synchrotron X-ray Topographic Investigation of Electromagnetic Breadown Induced in p-n Junctions on Silicon", Guvernment Microcircuit Applications Conference Digest of Papers, Vol. XVI, 651-54, (1990) - Restricted Distribution.

Dudley, M., Wang, F. F. Y., Fanning, T., and Gordon-Smith, D., "Synchrotron X-ray Topography as a Non-Destructive Testing (NDT) Monitor of I.C. Processing", in Materials Reliability Issues in Microelectronics, ed. by M. L. Green, J. C. Gelpey, J. Worthman and R. Singh, Mat. Res. Soc. Symp. Proc., 224, 61-66, (1991). 
Dudley, M., Wang, F. F. Y., Fanning, T., Tolis, G., and Wu, J., "Synchrotron Topographic Studies of the Influence of Rapid Thermal Annealing on Defect Structures in Single Crystal Silicon", in Defects in Materials, ed. by P. D. Bristow, J. E. Epperson, J. E. Griffith and Z. Liliental-Weber (Eds.), Mat. Research Soc. Symp. Proc., 209, 511-16, (1991).

Dudley, M., Wang, F. F. Y., Fanning, T., Tolis, G., Wu, J., and Hodul, D. T., "Synchrotron X-ray Topographic Studies of the Changes in Defect Microstructure Induced by Rapid Thermal Processing of Silicon", Mater. Letts., 10, 87-92, (1990).

Dudley, M., Wu, J., Yao, G.-D., Liu, H.-Y., and Kao, Y. C., "Determination of 3-Dimensional Defect Structures in Gallium Arsenide Epilayers on Silicon Using White Beam Synchrotron Radiation Topography in Both Transmission and Grazing Bragg-Laue Geometry", in Layered Structures, Heteroepitaxy, Superlattices, Strain and Metastability, ed. by B. W. Dodson, L. J. Schowalter, J. E. Cunningham and F. H. Pollak, Mat. Research Soc. Symp. Proc., 160, 469-474, (1990).

Dudley, M., Yao, G.-D., Paine, D., Howard, D., and Sacks, R. N., "A Characterization of $\operatorname{In}_{x}$ Ga1-x As Strained Layer Systems Using a Combination of TEM and Synchrotron X-ray Topography", Mater. Sci. \& Engin. B, 10, 75-84 (1991).

Dudley, M., Yao, G.-D., Paine, D., Howard, D., and Sacks, R. N. "Combined TEM and X-ray Topographic Characterization of In $\mathrm{Ial}_{-\chi}$ As Strained Layer Systems", in Defects in Materials, ed. by P. D. Bristowe J. E. Epperson, J. E. Griffith and Z. Liliental-Weber, Mat. Research Soc. Symp. Proc., 209, 655-660, (1991).

Dudley, M., Yao, G.-D., and Wu, J., "Characterization of Superconductor Heterostructures Using Synchrotron X-ray Topography", Advances in X-ray Analysis, 35, (1991).

Dudley, M., Yao, G.-D., and Wu, J., "Investigation of Semiconductor Heterostructures by Synchrotron White Beam X-ray Topography in Grazing Bragg-Laue Geometries", Advances in X-ray Analysis, 35, (1991).

Dudley, M., Yao, G.-D., Wu, J. and Liu, H.-Y., "Depth Sensitive Imaging of Defects in Epilayers and Single Crystals Using White Beam Synchrotron Radiation Topography in Grazing Bragg-Laue Geometry", in Impurities, Defects and Diffusion and Semiconductors: Bulk and Layered Structures, ed. by J. Bernholc, E. E. Haller and D. J. Wolford, Mat. Research Soc. Symp. Proc., 163, 1031-1036, (1990).

Fanning, T., Dudley, M., Wang, F. F. Y., Gordon-Smith, D., "Synchrotron X-ray Topography Study of Defects in High Carbon-Containing Si Wafers", in Rapid Thermal and Integrated Processing, ed. by J. R. Lloyd, T. S. Ho, C. T. Sah, and E. Host, Mat. Research Soc. Symp. Proc., 225, 301-06, (1991).

Jardine, A. P., Dudley, M., Yao, G.-D., and Tolis, G., "X-ray Topographic Analysis of Strain Fields Associated with MicronSized Grating on Si(100) Surfaces", in Layered Structure, Heteroepitaxy, Superlattices, Strain and Metastability, ed. by B. W. Dodson, L. J. Schowalter, J. E. Cunningham and F. H. Pollak, Mat. Res. Soc. Symp. Proc., 160, 533-38, (1990).

Liu, F., Baker, I., Yao, G.-D., and Dudley, M., "Dislocations and Grain Boundaries in Polycrystalline Ice: A Preliminary Study by Synchrotron X-ray Topography, J. Mater. Sci., (1991).

Milat, J. and Dudley, M., "X-ray Topography", Chapter 3 in Application of Synchrotron Radiation in Chemistry, ed. by C.R.A. Catlow and G. N. Greaves, Blackie and Son Ltd., Glasgow, U.K., (1990), 65-99.

Wang, Y., Liu, X., Yao, G.-D., Liebermann, R. C. and Dudley, M., "High Temperature TEM and X-ray Diffraction Studies on Twinning and the Phase Transition at $145^{\circ} \mathrm{C}$ in $\mathrm{LaGaO}_{3}$ ", Mater, Sci. \& Eng., A132, 13-21, (1990).

Winter, Jr., J. M., White Beam Transmission Topography of Single Crystal Gallium Arsenide, Ph.D. Thesis, The Johns Hopkins University, (1991).

Winter, Jr., J. M., Green, Jr., R. E., and Green, K. A., "Application of Synchrotron and Flash X-ray Topography to Improved Processing of Electronic Materials", Advances in X-ray Analysis, 35, (1991).

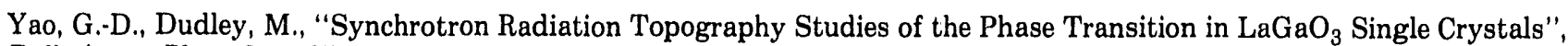
Bull. Amer. Phys. Soc., 35, 1792, (1990).

Yao., G.-D., Dudley, M., Hou, S.-Y. and DiSalvo, R., "Application of White Beam Synchrotron Radiation Topography to the Analysis of Twins", Nucl. Instr. \& Meth., 56/57, 400-04, (1991).

Yao, G.-D., Dudley, M., Wang, Y., Liu, X. and Liebermann, R.C., "Synchrotron Radiation Topography Studies of the Phase Transition in $\mathrm{LaGaO}_{3}$ Single Crystals", Nucl. Instr. \& Meth., 56/57, 405-09, (1991).

Yao, G.-D., Dudley, M., Wang, Y., Liu, X. and Liebermann, R. C., "Synchrotron X-ray Topographic Studies of Twinning and the Phase Transition at $145^{\circ} \mathrm{C}$ in $\mathrm{LaGaO}_{3}$ Single Crystals", Mater. Sci. \& Engin., A132, 23-30, (1990).

Yao, G.-D., Dudley, M. and Wu, J., "Synchrotron White Beam Topographic Imaging in Grazing Bragg-Laue Geometries", J. X-ray Science \& Technology, 2, 195-213, (1990).

Yao, G.-D., Wu, J., Dudley, M., Shastry, V. and Anderson P., "Influence of Surface Relaxiation on X-ray Topographic Imaging of Interfacial Dislocations in Heterosystems", in Defects in Material, ed. by P. D. Bristowe, J. E. Lpperson, J. E. Griffith and Z. Liliental-Weber, Mat. Res. Soc. Symp. Proc., 209, 707-12, (1991).

Yuan, D., and Dudley, M., "Disolcation Line Direction Determination in Pyrene Single Crystals", Mol. Cryst. Liq. Cryst., (1991). 


\section{Beamline X20}

Birgeneau, R. J., Aharony, A., Cowley, R. A., Hill, J. P., Pelcovits, R. A., Shirane, G. and Thurston, T. R., "Effects of Random Fields on Bicritical Phase Diagrams in Two and Three Dimensions", Physica, 177, 58, (1991).

Gibbs, D., Ocko, B. M., Zehner, D. M., and Mochrie, S. G. J., "Structure and Phases of the Au(001) Surface: In-Plane Structure", Phys. Rev. B 42, (12), 7330 (1990).

Factor, B. J., Grazing Incidence X-ray Scattering Studies of Thin Polymer Films, (Thesis, Stanford Univ., September 1991).

Factor, B. J., Russell, T. P., and Toney, M. F., "Surface-Induced Ordering of an Aromatic Polyimide", Phys. Rev. Lett., 66, $1181,(1991)$.

Hainsey, R. F., A Combined Thermodynamics and X-ray Scattering Study of the Krypton/Graphite System in the Multilayer Region, (Thesis, Carnegie-Mellon Univ., 1991).

Hainsey, R. F., Gangwar, R., Schindler, J. D., and Suter, R. M., "Reentrant Disordered Phase in Two-Layer Films of Krypton on Graphite", Phys. Rev. B 44, 3365, (1991).

Hill J. P., Thurston, T. R., Erwin, R. W., Ramstad, M. J., and Birgeneau, R. J., "Transition to Long-Range Order in the ThreeDimensional Random Field Ising Model", Phys. Rev. Lett., 66, (25), 3281, (1991).

Huang, K. G., Gibbs, D., Zehner, D. M., Sandy, A. R., and Mochrie, S. G. J., "Phase Behavior of the Au(111) Surface: Discommensurations and Kinks", Phys. Rev. Lett., 65, (26), 3313, (1990).

Keane, D. T., Bancel, P. A., Jordan-Sweet, J. L., Held, G. A., Mak, A., and Birgeneau, R. J., "Evidence for Two-Step Disordering of the Au(110) $1 \times 2$ Reconstructed Surface", Surf. Sci., 250, 8, (1991).

Köster, U., Schunemann, U., Blank-Bewersdorff, M., Brauer, S., Sutton, M., and Stephenson, G. B., "Nanocrystalline Materials by Crystallization of Metal-Metalloid Glasses", Materials Sci. and Engineering, A133, 611, (1991).

Mak, A., Synchrotron X-ray Diffraction Studies of Surface Disordering of Novel Metal and Germanium Surfaces, (Thesis, Massachusetts Inst. Technol., June 1991).

Mak, A., Evans-Lutterodt, K. W., Blum, K., Noh, D. Y., Brock, J. D., Held, G. A. and Birgeneau, R. J., "Synchrotron X-ray Study of the Disordering of the Ge(111) Surface at High Temperatures", Phys. Rev. Lett., 66, 2002, (1991).

Noh, D. Y., X-ray Scattering Study of Orientational and Positional Order in Liquid Crystal Films, (Thesis, Mass. Inst.

Technol, December, 1991).

Noh, D. Y., Brock, J. D., Fossum, J. O., Hill, J. P., Nuttall, W. J., Litster, J. D., and Birgeneau, R. J., "Synchrotron X-ray Study of Dimensional Crossover in Solid-Phase Smectic Liquid Crystal Films", Phys, Rev, B 43, 842, (1991).

Ocko, B. M., Huang, K. G., Gibbs, D., Zehner, D. M., and Mochrie, S. G. J., "Structure and Phases of the Au(001) Surface: Absolute Reflectivity", Phys. Rev. B 44, (12), 6429, (1991).

Rabedeau, T. A., Tidswell, I. M., Pershan, P. S., Bevk, J., and Freer, B. S., "X-ray Scattering Studies of the $\mathrm{SiO}_{2} / \mathrm{Si}(001)$ Interfacial Structure", Appl. Phys. Lett., 59, 706, (1991).

Samant, M. G., Brown, C. A., and Gordon, II, J. G., "Structure of an Ordered Self-Assembled Monolayer of Docosyl Thiol on Gold(111) by Surface X-ray Diffraction", Langmuir, 7, 437, (1991).

Sandy, A. R., Mochrie, S. G. J., Zehner, D. M., Huang, K. G., and Gibbs, D., "Structure and Phases of the Au(111) Surface: Xray Scattering Measurements", Phys. Rev. B 43, (6), 4667, (1991).

Tidswell, I. M., X-ray Scattering from Silicon and Thin Films on Silicon, (Thesis, Harvard Univ., 1991).

Tidswell, I. M., Rabedeau, T. A., Pershan, P. S., Kosowsky, S. D., Folkers J. P., and Whitesides, G. M., "X-ray Grazing Incidence Diffraction from Alkylsiloxane Monolayers on Silicon Wafers", J. Chem. Phys., 95, 2854, (1991).

Toney, M. F., "In-situ Synchrotron X-ray Diffraction of Electrochemical Interfaces", Proc. Symp. on Applications of Surface Analysis Methods to Erivironmental/Materials Interactions, ed. by D. R. Baer, C. R. Clayton and G. D. Davis, The Electrochemical Society, Pennington, 1991, p. 200.

Toney, M. F., Gordon II, J. G., Samant, M. G., Borges, G. L., Melroy, O. R., Kau, L. S., Wiesler, D. G., Yee, D., and Sorensen, L. B., "Surface X-ray Scattering Measurement of the Substrate-Induced Spatial Modulation of an Incommensurate Absorbed Monolayer", Phys. Rev. B 42 (2), 5594, (1990).

Toney, M. F., Gordon, J. G., Samant, M. G., Borges, G. L., Wiesler, D. G., Sorensen, L. B., and Yee, D., "In-situ Surface X-ray Scattering Measurements of Electrochemically Deposited Bi on Ag(111): Structure Compressibility, and Comparison with Exsitu LEED Measurements", Langmuir, 7, 796, (1991).

Yang, Y. S., Time-Resolved X-ray Scattering Study of Crystallization In Zirconium-Based Glasses, (Thesis, McGill University, June, 1990).

Zehner, D. M., Mochrie, S. G. J., Ocko, B. M., and Gibbs, D., "Structure and Phases of the Au(001) Surface: X-ray Scattering Measurements", J. Vac. Sci. Tech., 9, (3), 1861, (1991). 


\section{Beamline X22B}

Ocko, B. M., Gibbs, D., Huang, K. G., Zehner, D. M. and Zehner, S. G. J., "Structure and Phases of the Au(001) Surface: Absolute X-Ray Reflectivity", Phys. Rev. B 44,6429, (1991).

Pershan, P. S., "Structure of Surfaces and Interfaces as Studied Using Synchrotron Radiation: Liquid Surfaces", Faraday Discuss., Chem. Soc., 89, 231, (1990).

Sanyal, M. K., Sinha, S. K., Huang, K. G., Ocko, B. M., "X-ray Scattering Studies of Capillary Wave Fluctuations at a Liquid Surface", Phys. Rev. Lett., 66, 628-31, (1991).

Schlossman, M. L., Schwartz, D. K., Kawamoto, E. H., Kellogg, G. J., Pershan, P. S., Kim, M. W., and Chung, T. C., "X-ray Reflectivity of a Polymer Monolayer at the Water/Vapor Interfaces", J. Phys. Chem., 95, 6628, (1991).

Schlossman, M. L., Schwartz, D. K., Kawamoto, E. H., Kellogg, G. J., Pershan, P. S., Ocko, B. M., Kim, M. W., and Chung, T. C., "X-ray Studies of the Liquid/Vapor Interface: Water and Polymer and Fatty Acid Monolayers on Water", Mat. Res. Soc. Symp. Proc., 177, 351 (1991).

Schlossman, M. L., Schwartz, D. K., Pershan, P. S., Kawamoto, E. H., Kellogg G. J., and Lee, S., "Relaxation and Appearance of a Reentrant Phase in a Molecular Monolayer", Phys. Rev. Lett., 66, 1599, (1991).

Sinha, S. K., Sanyal, M. K., Huang, K. G., Ocko, B. M.. "X.ray Scattering Studies of Capillary Fluctuations at Liquid Surfaces and in Thin Liquid Films", Proc. of Mat. Res. Soc. Fall Meeting, (1990).

Swislow, G., Schwartz, D., Ocko, B. M., Pershan, P. S., and Litster, J. D., "X-ray Studies of the Surface and Bulk Structure of the Isotropic and Nematic Phase of a Lyotropic Liquid Crystals", Phys. Rev. A 43, 6815, (1991).

You, H., Welp, U., Fang, Y., "Slope Discontinuity and Fluctuation of Lattice Expansion Near Tc in Untwinned $\mathrm{YBa}_{2} \mathrm{Cu}_{3} \mathrm{O}_{7-8}$ Single Crystals", Phys. Rev. B, 43, 3660, (1991).

\section{Beamline X22C}

Bohr J., Gibbs D., Huang, K., "X-ray Diffraction Studies of the Magnetic State of Thulium", Phys. Rev. B, 42, 4322, (1990).

Gibbs, D., Grubbel, G., Harshman, D. R., Isaacs, E. D., McWhan, D. B., Mills, D., Vettier, C., "Polarization and Resonance Studies of X-ray Magnetic Scattering in Holmium", Phy's, Rev. B 43, 5663- 81, (1991).

Grübel, G., Axe, J. D., Gibbs, D., Lander, S. H., Marineggi, J. C., Brückel, T., "Incommensurate Charge-Density-Wave State in $\alpha$-Uranium - A High Resolution X-ray and Neutron Scattering Study", Phys. Rev. B 43, 8803, (1991).

Majkerzak, C. F., Kwo, J., Houg, M., Yafet, Y., Gibbs, D., Chien, C. L., Bohr, J., "Magnetic Rare Earth Superlattices", Adv. in Phys., 40, 99, (1991).

Ocko, B. M., Gibbs, D., Huang, K. G., Zehner, D. M., Mochvie, S., "Structure and Phases of the Au(001) Surface: Absolute X-ray Reflectivity", Phys. Rev. B, 44, (1991).

Sinha, S. K., Sanyal, M. K., Gibaud, A., Satija, S. K., Majkrzak, C. F., Homma, H., "The Characertization of Interface Roughness and Other Defects in Multilayers by X-ray Scattering", NATO Advanced Study Institute Conf., June 26-July 6, 1990, Crete, Greece.

Sanyal, M. K., Sinha, S. K., Huang, K. G., Ocko, B. M., "X-ray Scattering Study of Capillary - Wave Fluctuations at a Liquid Surface", Phys. Rev. Lett., 66, 628, (1991).

Zehner, D. M., Mochrie, S., Ocko, B. M., Gibbs, D., "Structure and Phases of the Au(001) Surface: X-ray Scattering Measurements", J. Vac. Sci. Tech., A9, (3), 1861, (1991).

\section{Beamline X23A2}

Bouldin, C. E., Forman, R. A., Bell, M. I., and Donovan, E. P., "Structural Relaxation in Ion-Damaged Ge", Phys. Rev. B.

Kendelewicz, T., Woicik, J. C., Miyano, K. E., Cowan, P. L., Karlin, B. A., Bouldin, C. E., Pianetta, P., and Spicer, W. E., "Synchrotron X-ray Standing-Wave Study of Sb on GaAs(110) and InP(110)", J. Vac. Sci. Tech.

Kim, K. H., Bell, M. I., Dozier, C. M., Freitag, R. K., and Bouldin, C. E., "Correcting for the X-ray Energy Calibration Error Caused by Misalignment of a Right-Angle Linkage Monochromator", Rev. Sci. Inst., 62, 982, (1991).

Webb, A. W., Kim, K. H., and Bouldin, C. E., "The Valence of Copper in $\mathrm{LaCuO}_{3}$ : An X-ray Absorption Study", Solid State Comm.

Woicik, J. C., Bouldin, C. E., Cross, J. O., Sorensen, L. B., King, C. A., "EXAFS and X-ray Diffraction Study of Strain and Bond Distortions in Epitaxial Semiconductor Layers", J. Vac. Sci. Tech.

Woicik, J. C., Kendelewicz, T., Miyano, K. E., Bouldin, C. E., Meissner, P. L., Pianetta, P., and Spicer, W. E., "Structure of the $\mathrm{Si}(111) \mathrm{Si}(111) \sqrt{3} \times \sqrt{3} \mathrm{Sb}$ Interface by SEXAFS and Photoemission", J. Vac. Sci. Tech. 


\section{Beamline X23A3}

Black, D. R., Burdette, H. E., Dobbyn, R. C., Spal, R. D., and Kuriyama, M., "Advanced X-ray Imaging Methods for Ceramics", American Ceramic Society Bulletin, 70, 1004-1009, (1991).

Black, D. R., Burdette, H. E., Kuriyama, M., and Spal, R. D., "Diffraction Imaging of Polycrystalline Materials", J. Material Research, 6, 1469, (1991).

Jemian, P. R., and Long, G. G., "Silicon Photodiode Detector for Small-Angle X-ray Scattering", J. Appl. Cryst., 23, 430-432, (1990).

Kuriyama, M., Dubbyn, R., Burdette, H. E., Spal, R., and Black, D., "Hard X-ray Microscope with Submicron Spatial Resolution", J. Res. NIST, 95, 559-574, (1990).

Long, G., Jemian, P., Weertman, G., Black, D., Burdette, H., and Spal, R., "High Resolution Small-Angle X-ray Scattering Camera for Anomalous Scattering", J. Appl. Cryst., 24, 30-37, (1991).

Long, G. G., Krueger, S., Black, D. R., Cline, J. P., Jemian, P. R., and Gerhardt, R. A., "Small-Angle Neutron Scattering and Small-Angle X-ray Scattering From Bulk Microporous Silica", Synch. Rad. in Material Res., M. R. S. Symp. Proc., 166, 421426, (1990).

Mendoza, E. A., Sunil, D., Wolkow, E., Gafney, H. D., Rafailovich, M. H., Sokolov, J., Long, G. G., Jemian, P. R., Schwartz, S. A., and Wilkens, B. J., "Effect of Photodeposited Iron Oxide and Tin Oxide on the Consolidation of Porous Vycor Glass", Appl. Phys. Lett., 57 (3), 209, (1990).

Posthill, J. B., Rudder, R. A., Hudson, G. C., Malta, D. P., Fountain, G. G., Thomas, R. E., Markunas, R. J., Humphreys, T. P., Nemanich, R. J., and Black, D. R." "Substrate Effects and the Growth of Homoepitaxial Diamond(100) Layers Using Low Pressure rf Plasma Enhanced Chemical Vapor Deposition", Proc. of the 2nd International Symposium on Diamond Materials, Washington, D.C., (1991), 274.

Steiner, B., Dobbyn, R., "Crystal Regularity with High-Resolution Synchrotron X-Radiation Diffraction Imaging", American Ceramic Soc. Bulletin, 70, 1017-1023, (1991).

Steiner, B., Dobbyn, R., Black, D., Burdette, H., Kuriyama, M., Spal, R., van den Berg, L., Fripp, A., Simchick, R., Lal, R., Barta, R., Matthiesen, D., and Ditchek, B., "High Resolution Synchrotron X-Radiation Diffraction Imaging of Crystals Grown in Microgravity and Closely Related Terrestrial Crystals", Proceedings of the SPIE'S International Symposium on Optical Applied Science and Engineering, July 21-26, San Diego, CA, (1991).

Steiner, B., Dobbyn, R., Black, D., Spal, R., van den Berg, L., Simchick, R., Fripp, A., Batra, A., Lal, R., Matthiesen, D., Ditchek, B., and Kuriyama, M., "High Resolution Synchrotron X-Radiation Diffraction Imaging of Crystals Grown in Microgravity and Closely Related Terrestrial Crystals", J. Res. NIST, 96, 305-331, (1991).

\section{Beamline X23B}

Harris, V. G., Oliver, S. A., Nowak, W. B., Kim, K. H., Elam, E. T., and Vittoria, C., "Correlation of Magnetic Properties and Local Atomic Structure Observed by Electron Yield Extended X-ray Absorption Fine Structure in $\mathrm{Amorphous}^{\mathrm{Co}_{74}} \mathrm{Fe}_{6} \mathrm{~B}_{16} \mathrm{Si}_{5}$ Thin Films", J. Appl. Phys. 69, 1-4, (1991).

Harris, V. G., Oliver, S. A., Nowak, W. B., Vittoria, C., Kim, K. H., and Elam, W. T., "Correlation of Magnetic Properties and Local Atomic Structure Observed by Electron-Yield EXAFS in Heat-Treated Amorphous $\mathrm{Co}_{74} \mathrm{Fe}_{6} \mathrm{~B}_{15} \mathrm{Si}_{5} \mathrm{Films}$ ", J. Appl. Phys., 69, (1), 1, (1991).

Kim, K. H., Bell, M. I., Dozier, C. M., Freitag, R. K., and Bouldin, C. E., "Correcting for X-ray Calibration Error Caused by Misalignment of a Right-angle Linkage Monochromator", Rev. Sci. Instrum. 62, 1-4, (1991).

Woicik, J. C., Kendelewicz, T., Miyano, K. C., Bouldin, C. E., Meissner, P. L., Pianetta, and Spicer, W. E., "Local Bonding Structures of $\mathrm{Sb}$ on $\mathrm{Si}(111)$ by Surface Extended X-ray Absorption Fine Structure and Photoemission", Phys. Rev. B 43, 4331$4339,(1991)$.

\section{Beamline X24A}

Cowan, P. L., "Polarization and Anisotropy of X-ray Emission from Molecules", in X-Ray and Inner-Shell Processes, AIP Conf. Proceedings 215, ed. by T. A. Carlson, M. O. Krause, and S. T. Manson, (American Inst. of Physics, New York, 1991), pp. 696-709.

Cowan, P. L., "Selective Excitaion of X-ray Emission Spectra", Physica Scripta, T31, 112-118, (1990).

Cowan, P. L., Lindle, D. W., Southworth, S. H., Karlin, B. A., Perera, R. C. C., "Use of Multilayers in a Double Crystal Monochromator", Nucl. Instrum. Meth. A291, 219-220, (1990).

Kendelewicz, T., Woicik, J. C., Miyano, K. E., Cowan, P. L., Karlin, B. A., Bouldin, C. E., Pianetta, P., and Spicer, W. E., "Synchrotron X-ray Standing.Wave Study of Sb on GaAs(110) and InP(110)", J. Vac. Sci. Tech. B9, 2290, (1991). 
Levin, J. C., Biedermann, C., Keller, N., Liljeby, L., O, C.-S., Short, R. T., Sellin, I. A., and Lindle, D. W., "Auger-Photoion Auger-Electron Coincidence Measurements Following K-Shell Excitation by Synchrotron Radiation", Phys. Rev. Lett. 65, 988, (1990).

Levin, J. C., Lindle, D. W., Keller, N., Miller, R. D., Azuma, Y., Berrah Mansour, N., Berry, H. G., Sellin, I. A., "Measurement of the Ratio of Double-to-Single Photoionization of Helium at $2.8 \mathrm{keV}$ Using Synchrotron Radiation", Phys. Reu. Lett., 67, 968, (1991).

Lindle, D. W., Cowan, P. L., Jach, T., LaVilla, R. E., Deslattes, R. D., "Polarized X-Ray Emission Studies of Methyl Chloride and the Chlorofluoromethanes", Phys. Rev. A 43, 2353, (1991).

Mayer, R., Lindle, D. W., Southworth, S. H., and Cowan, P. L., "Direct Determination of Molecular Orbital Symmetry of $\mathrm{H}_{2} \mathrm{~S}$ Using Polarized X-ray Emission", Phys, Rev, A 43, 235, (1991).

Perera, R. C. C., Cowan, P. L., Lindle, D. W., LaVilla, R. E., Jach, T., and Deslattes, R. D., "Molecular Orbital Studies via Satellite Free X-ray Fluorescence: $\mathrm{Cl}(\mathrm{K} \cdot \mathrm{V})$ Emission and K Adsorption Spectra of Chlorofluoromethanes", Phys. Rev. A 43, 3609, (1991).

Southworth, S. H., Lindle, D. W., Mayer, R., and Cowan, P. L., "Anisotropy of Polarized X-ray Emission From Atoms and Molecules", Nucl. Instr. and Meth. B 56/57, 304, (1991).

Southworth, S. H., Lindle, D. W., Mayer, R., and Cowan, P. L., "Anisotropy of Polarized X-ray Emission From Molecules", Phys. Rev. Lett., 67, 1098, (1991).

Woicik, J. C., Kendelewicz, T., Miyano, K. E., Cowan, P. L., Bouldin, C. E., Karlin, B. A., Pianetta, P., and Spicer, W. E., "Determination of the Sb/Si(111) Interfacial Structure by Back-Reflection X-ray Standing Waves and Surface Extended X-ray Absorption Fine Structure", Phys, Rev. B 44, 3475, (1991).

\section{Beamline X24C}

Barbee, Jr., T. W., Rife, J. C., Hunter, W. R., Cruddace, R. G., and Pianetta, P., "Multilayer Diffraction Gratings: Application to Synchrotron Radiation Instrumentation", Opt. Engr. 29, 738, (1990).

Benitez, E. L., Husk, D. E., Tarrio, C., and Schnatterly, S. E., "Surface Recombination Effects in Soft X-ray Efficiencies", Appl. Phys. Lett., 59, 396, (1991).

Goldenberg, S. S., Long, J. P., and Kabler, M. N., "Photoemission Study of Cleaved GaAs Surfaces Modified by Weak Laser Irradiation Using Combined Laser and Synchrotron Radiation", Proc. of 20th World Scientific International Conf. on the Physics of Semiconductors, ed. by E. M. Anastassakis, J. D. Joannopoulos, (1991), p. 139.

Goldenberg, S. S, Long, J. P., and Kabler, M. N., "Photoemission Study of Low-Flu'dnce, Nonthermal Laser Damage of UHVCleaved Gallium Arsenide (110)", in Surface Chemistry and Beam-Solid Interactions, ed. H. A. Atwater, F. A., Houle, D. H. Lowndes, Mater. Res. Soc. Symp. Proc. 201, (1991), p. 519.

Husk, D. E., Tarrio, C., Benitez, E. L., and Schnatterly, S. E., "Response of Photodiodes in the Vacuum Ultraviolet", J. Appl. Phys., 70, 3338, (1991).

\section{Beamline X25}

Berman, L. E., and Hart, M., "Adaptive Crystal Optics for High Power Synchrotron Sources", Nucl. Instrum. Methods Phys. Res., Sect. A 302, 558, (1991).

Berman, L. E., and Hart, M., "Performance of Water Jet Cooled Silicon Monochromators on a Multipole Wiggler Beam Line at NSLS", Nucl. Instrum. Methods Phys. Res., Sect. A 300, 415, (1991).

Berman, L. E., and Hart, M., "Water Jet Cooled Silicon Monochromators", Synchrotron Radiation News 4, No. 1, 22, (1991).

Sutton, M., Mochrie, S. G. J., Greytak, T., Nagler, S. E., Berman, L. E., Held, G. A., and Stephenson, G. B., "Observation of Speckle by Diffraction with Coherent X-rays"', Nature, 352, 608, (1991).

Zeman, H. D., Dilmanian, F. A., Garrett, R. F., Berman, L. E., Chapman, L. D., Hastings, J. B., Oversluizen, T., Siddons, D. P., Stojanoff, V., and Thomlinson, W. C., "An X-ray Monochromator for Dual-Energy Computerized Tomography Using Synchrotron Radiation", Nuclear Instrum. Methods Phys. Res., Sect. B56/57, 1218, (1991).

\section{Beamline X26A,C}

Bockman, R. S., Warrell, Jr., R. P., Levine, B., Pounds, J. G., Schidlovsky, G., and Jones, K. W., "Trace Elemental Analysis in Bone Using X-ray Microscopy", In Vivo Body Composition Studies, Recent Advances, ed. by S. Yasumara, J. E. Harrison, K. G, McNeill, A. D. Woodhead, and F. A. Dilmanian, (Plenum Press, New York, 1990), pp. 293-6. 
Church, D. A., Kravis, S. D., Johnson, B. M., Azuma, Y., Levin, J. C., Sellin, I, A, Meron, M., Jones, K. W., Druetta, M., Mansour, N., Berry, H. G., and Short, R. T. "Electron Transfer Collision Studies on Stored Ions Produced by Synchrotron Radiation", Nucl. Instrum. Methods B56/57, 417-21, (1991).

Conner, W. C., Webb, S. W., Spanne, P., Jones, K. W. "The Use of X-ray Microscopy and Syuchrotron Microtomography to Characterize Polyethylene Polymerization Particles", Macromolecules 23, 4742-7, (1990).

Flynn, G. J., and Sutton, S. R., "Chemical Characterization of Seven Large Area Collector Particles by SXRF", Proc. Lunar" and Planetary Sclence, Vol. 21, 549-56, (1991).

Flynn, G. J., and Sutton, S. R., "Cosmic Dust Particle Densities: Evidence for Two Populations of Stony Micrometeorites", Proc. Lunar and Planetary Science, Vol, 21, 541-47, (1991).

Gordon, B. M., Jones, K. W., "Synchrotron Radiation and its Application 'o Chemical Speclation: A Review", in Biological Trace Element Research, American Chemical Society Symposium Series No, 445, ed. by K, S. Subramanian, G. V. Yenger, II, and K. Okamoto, (American Chemical Society, Washington, D.C., 1991), Chpt. 23, 290-305.

Johnson, B. M., "Atomic Physics Research with Second and Third Generation Synchrotron Light Sources", Nucl. Instrum. Methods B56/57, 433-7, (1991).

Jones, K. W., Spanne, P., Webb, S. W., Conner, W. C., Beyerlein, R. A., Reagan, W. J., and Dautzenberg, F. M., "Catalyst Analysis Using Synchrotron X-ray Microscopy", Nucl. Instrum. Methods B56/57, 427-32, (1991).

Jones, K. W., Schidlovsky, G., Burger, D. E., Milder, F, L., and Hu, H., "Distribution of Lead in Human Bone: III. Synchro" tron X-ray Microscope Measurements", In Vivo Body Composition Studies Recent Advances, ed. by S. Yasumura. J. E. Harrison, K. G. McNeill, A. D. Woodhead, F. A. Dilmanian, (Plenum Press, N.Y., 1990), pp, 281-6.

Kravis, S. D., Church, D. A., Johnson, B. M., Levin, J. C., Azuma, Y., Sellin, I. A, Meron, M., Jones, K. W., Druetta, M., Mansour, N., Berry, H. G., Short, R. T., "Sequential Photoionization of Ions Using Synchrotron Radiation and a Penning Ion Trap", Nucl. Instrum. Methods B56/57, 396-9, (1991).

Kravis, S. D., Church, D. A., Johnson, B. M., Meron, M., Jones, K. W., Levin, J., Sellin, I. A., Azuma, Y., Berra Mansour, N., Berry, H. G., Druetta, M., "Inner-shell Photoionization of Stored Positive lons Using Synchrotron Raditaion", Phys. Rev. Let.t, 66, 2956-9, (1991).

Lowenstern, J. B., Mahood, G. A., Rivers, M. L., Sutton, S. R., "Evidence for Extreme Partitioning of Copper into a Magmatic Vapor Phase", Science, 252, 1405-9, (1991).

Rivers, M. L., Sutton, S. R., Jones, K. W., "Synchrotron X-ray Fluoresence Microscopy", Synch. Rad. News 4, (2), 23-6, (1991). Tuniz, C., Zanini, F., Jones, K. W., "Probing the Environment with Accelerator-Based Techniques", Nucl. Instrum. Methods B56/57, 877-81, (1991).

Wu, Y., Thompson, A. C., Underwood, J. H., Giauque, R. D., Chapman, K., Rivers, M. L., and Jones, K. W., "A Tunable X-ray Microprobe Using Synchrotron Radiation", Nucl. Instrum. Methods A291, 146-51, (1990). 


\section{Publications of the NSLS Staff}

Ade, H, Kirz, J., Hulbert, S., Johnson, E., Anderson, E., Kern, D., "Images of a Microelectronic Device with the X1-SPEM, a First Generation Scanning Photoemission Microscope at the NSLS", J, Vac. Sci. Tech. A9, 1902 (1991), BNL 45610.

Batchelor, K., Ben-Zvi, I., Fernow, R., Gallardo, J., Kirk, H, Pellegrini, C., van Steenbergen, A., Bhowmik, A., "A Microwiggler Free Electron Laser at the Brookhaven Accelerator Test Facility", Nucl. Instr, \& Meth. in Phys. Res., A296, (1990), 239-243, BNL 46639.

Ben-Zvi, I." "The BNL Accelerator Test Facillty and Experimental Program", Conference Record of the 1991 IEEE Particle Accelerator Conference, May 6-9, 1991, Vol, 1, pp. 550-54, (1991), BNL 46641, September 1991.

Ben-Zvi, I, DiMauro, F., Krinsky, S., White, M. G. and Yu, L, H." "Proposed UV-FEL User Facility at BNL", Nucl. Instr, and Meth., A304, 181, (1991), BNL 45161.

Ben-Zvi, I., Lombardi, A., Paul, P., "Design of a Superconducting RFQ Resonator", Particle Accelerators, Vol. 35, 177-192, (1991), BNL 46607.

Ben-Zvi, I., Xie, J., Zhang, R., "Feed Forward RF Control System of the Accelerator Test Facility", Conference Record of the 1991 IEEE Particle Accelerator Conference, Vol. 2, May 6-9, 1991, pp. 1323-2,5, (1991), BNL 46643.

Berman, L. E., Hart, M., "Performance of Water Jet Cooled Silicon Monochromators on a Multipole Wiggler Beam Line at NSLS", Nucl. Instr, \& Meth, in Phys. Res., Section A 300, 415, (1991), BNL 45530.

Berman, L. E., Hart, M., "Water Jet Cooled Silicon Monochromators", Synchrotron Rad. News, Vol. 4, No. 1, pg. 22, (1991), BNL 45531.

Berman, L. E., Hart, M., "Adaptive Crystal Optics for High Power Synchrotron Sources", Nucl. Instr, \& Meth. in Phys. Res., Section A 302, 658, (1991), BNL 45609.

Berman, L. E., Hastings, J. B., Oversluizen, T., Woodle, M., "Optical Design and Performance of the X25 Hybrid Wiggler Beam Line at the NSLS", Rev. Sci. Instrum., 63, 428, (1992), BNL 46654.

Berman, L. E., Hart, M., "Performance of Water Jet Cooled Silicon Monochromators in High Power X-Ray Beams", Rev. Sci. Instrum. 63 (1), 437, (1992), BNL 46652.

Biscardi, R., Broome, W., Buda, S., Keane, J., Ramirez, G., Wachtel, J., Wang, J. M., "Progress on Bunch Lengthening at the NSLS VUV Ring", Conference Record of the 1991 IEEE Particle Accelerator Conference, Vol. 2, May 6-9, 1991, pp. 1326-28, (1991), BNL 46179.

Blumberg, L. N., Murphy, J. B., Sharma, S., "Orbits, Tunes and Chromaticities for the BNL SXLS Storage Ring", Conference Record of the 1991 IEEE Particle Accelerator Conference, May 6-9, 1991, Vol, 4, pp. 2661-663, (1991), BNL 46184.

Blumberg, L. N., Murphy, J. B., Reusch, M., "Lattice Properties of the Phase I BNL X.Ray Lithography Source Obtained from Fits to Magnetic Measurement Data", Conference Record of the 1991 IEEE Particle Accelerator Conference, May 6-9, 1991, Vol, 4, pp. 2664-666, (1991).

Bohr, J., Gibbs, D., Sinah, S. K., Krätschmer, W., Van Tendeloo, G., Larsen, E., Egsgaard, H., Berman, L. E., "Order, Dis• order and Structure of Crystals of $\mathrm{C}_{60} / \mathrm{C}_{70}$ ", Europhys. Lett., 17 (4), 327-332, (1992), BNL 46503.

Bozoki, E., "Beam Transport to the SXLS Ring", Conference Record of the 1991 IEEE Particle Accelerator Conference, May 6-9, 1991, Vol. 5, p. 2703, (1991), BNL 46187.

Bozoki, E., "Calculation of Closed Orbit Errors Due to Misalignment of Combined Function Magnets with Large Bend Angle", Proceedings of EPAC 90 2nd European Part. Accel. Conf., Vol. 2, p. 1441, (1990), BNL 44656.

Bozoki, E., "Determination of Beam Position From Induced Electric Signals", Nucl. Instr, \& Meth., A307, 195, (1991), BNL 45582 .

Bozokl, E., Friedman, A., Ben-Zvi, I., "ASAP - A Symbolic Algebra Package for Accelerator Design", Conference Record of the 1991 IEEE Particle Accelerator Conference, May 6-9, 1991, Vol. 2, p. 272, (1991), BNL 46186.

Bozoki, E. and Halama, H., "Ion Related Problems for the XLS Ring", NIM, A307, 156 (1991), BNL 4.4523.

Bozoki, E, and Halama, H., "Ion Clearing in the XLS Ring", Proceedings of EPAC 90 2nd European Part. Accel. Conf., Vol. 1, 812 (1990), BNL 44657.

Budhani, R. C., Lesyna, L., DiMarzio, D., Wiesmann, H., Williams, G. P., "Electrodynamics of $\mathrm{YBa}_{2} \mathrm{Cu}_{3} \mathrm{O}_{7} \mathrm{Films}$ Deduced From Submillimeter Synchrotron Radiation Transmittance Measurements", Phys. Rev., 44, 7087, (1991), BNL 46595.

Carr, G, L., Quijada, M., Tanner, D. B., Hirschmugl, C. J., Williams, G, P., Etemad, S., DeRosa, F., Venkatesan, T., Dutta, B., Hemmick, D., Xi, X, "Infrared Response of $\mathrm{YBa}_{2} \mathrm{Cu}_{3} \mathrm{O}_{7-\delta}$ Filma to Pulsed, Broadband Synchrotron Radiation", SPIE 1292, 187, (1990), BNL 44573. 
Carr, G, L., Quijada, M., Tanner, D. B., Hirschmugl, C. J., Williams, G. P., Etemad, S., Dutta, B., DeRosa, F., Venkatesan, T., "Fast Bolometric Response by High ' $\mathrm{T}$ Detectors Measured with Subnanosecond Synchrotron Radiation", Appl. Phys, Lett., 57, 2725 (1990), BNL 45529.

Chapman, D., Moulin, H., Garrett, R. F., "Monochromator Harmonic Content Measurements and Calculations", Rev, Sci. Instrum., 63, (1), 893-95, (1992), BNL 46647.

Chou, T, S., "Photon Stimulated Desorption from Aluminum and Stainless Steel", J. Vac. Sci. Tech., A9, 2014, (1991), BNL 46468 .

Chou, T, S., "Photon Stimulated Desorption of Neutral Species from Aluminum", Conference Record of the IEEE Particle Accelerator Conference, May 6-9, 1991, Vol, 4, p. 2286, (1991) BNL 46474.

Cramer, S. P. deGroot, F. M. F., Ma, Y., Chen, C. T., Sette, F., Kipke, C. A., Elchhorn, D. M., Chan, M. K., Armstrong, W. H., Libby, E., Christou, G., Brooker, S., McKee, V., Mullins, O. C., Fuggle, J, C., "Ligand Field Strengths and Oxidation States from Manganese L-Edge Spectroscopy". Submitted to the Journal of American Chemical Society. BNL 45362, October 1990.

Desmond, E., Galayda, J., Louie, W., Rose, J., "Control System of the Superconducting X-Ray Lithography Source (SXLS) at Brookhaven", IEEE 1991 Particle Accelerator Conference Record, May 6-9, 1991, Vol, 3, pp. 1374-76, (1991), BNL, 45569.

Dilmanian, F. A., Garrett, R. F., Thomlinson, W. C., Berman, L. E., Chapman, L. D., Gmür, N. F., Lazarz, N. M., Luke, P. N., Moulin, H. R., Oversluizen, T., Slatkin, D. N., Stojanoff, V., Thompson, A. C., Volkow, N. D., and Zeman, H. D., "Multiple Energy Computed Tomography for Neuroradiology with Monochromatic X-Rays from the National Synchrotron Light Source", Physica Medica VI, 301, December, (1990), BNL 46750.

Dilmanian, F. A., Garrett, R. F., Thomlinson, W. C., Berman, L. E., Chapman, I, D., Hastings, J. B., Luke, P. N., Oversluizen, T., Siddons, D. P., Slatkin, D. N., Stojanoff, V., Thompson, A. C., Volkow, N. D., Zeman, H. D., "Computed Tomography with Monochromatic X-Rays from the National Synchrotron Light Source", Nucl. Instr. \& Meth. in Phys. Res., B56/57, 1208-1213, (1991), BNL 45598.

Forro, L., Carr, G. L., Williams, G. P., Mandrus, D., Mihaly, L., "Far Infrared Transmission Study of Single Crystal $\mathrm{Bi}_{2} \mathrm{Sr}_{2} \mathrm{Ca}_{1} \mathrm{Cu}_{2} \mathrm{O}_{\mathrm{x}}$ Superconductors", Phys. Rev. Lett. 65, 1941, (1990), BNL 45203.

Forest, E., Murphy, J., Reusch, M. F., "Explicit LIE Group Integrators", AIP Conforence Proceedings 230, Nonlinear Dynamics and Particle Acceleration, September 1991, 60-66, BNL 46712.

Friedman, A., "Reduction of Undulator Radiation and FEL Small Gain Due to Wiggler Errors", Conference Record of the 1991 IEEE Particle Accelerator Conference, May 6-9, 1991, Vol. 5, pp. 2766-68, (1991), BNL 46181.

Fureulid, L. R., Renner, M. W., and Fajer, J., "EXAFS Studies of Ni(II) and Ni(I) Factor 430M, Conformational Flexibility of F430 Skelton", J, Am. Chem. Soc. 112, 8987, (1990), BNL 44930.

Furenlid, L. R., Renner, M. W., Szalda, D. J., and Fuijita, E., "EXAFS Studies of Ni(II), Ni(I) and Ni(I)-CO T'etraazamacrocyles and the Crystal Structure of 5,7,7,12,14,14-Hexamethyl-1,4,8,11-tetraazacyclotetradeca-4,11-diene Nickel(I) perchlorate", J. Am. Chein. Soc. 113, 883 (1991), BNL 44843.

Galayda, J., Fauchet, A. M., "Operation of Synchrotron Light Sources with Multiple Insertion Devices", Conference Record of the 1991 IEEE Particle Accelerator Conference, May 6-9, 1991, Vol, 2, pp. 1099-1103, (1991), BNL 46680.

Gao, F., Carr, G. L., Porter, C. D., Tanner, D. B., Etemad, S., Venkatesan, T., Inam, A., Dutta, B., Wu, X.D., Williams, G. P., Hirschmugl, C. J., "Far Infrared Transmission and Reflection Studies of Oriented $\mathrm{YBa}_{2} \mathrm{Cu}_{3} \mathrm{O}_{7-\delta}$ Thin Films", Phys. Rev. B43, 10383, (1991), BNL 45254.

Garrett, R. F., Dilmanian, F. A., Oversluizen, T., Lenhard, A., Berman, L, E., Chapman, L. D., and Stoeber, W., "A High Energy Double Crystal Fixed Exit Monochromator for the X17 Superconducting Wiggler Beamline at the NSLS", Rev. Sci. Instrum., 63 (1), 594-598 (1992), BNL 46621.

Halama, H., Bozoki, E., "Ion Clearing and Photoelectron Production in the $200 \mathrm{MeV}$ SXLS Ring", IEEE 1991 Particle Accelerator Conference Record, May 6-9, 1991, Vol. 4, p. 2313, (1991), BNL 46471.

Halama, H. J., Guo, Y., "Non-evaporable Getter Investigation at the National Synchrotron Light Source", J. Vac. Sci. Tech. A9, 2070, (1991), BNL 46473.

Hämäläinen, K., Siddons, D. P., Hastings, J. B., Berman, L. E., "Elimination of the Inner Shell Lifetime Broadening in X-Ray Absorption Spectroscopy", Phys, Rev. Lett, 67, 2850, (1991), BNL 46601.

Happek, U., Sievers, A. J., Blum, E. B., "Observation of Coherent Transition Radiation;", Phys, Rev, Lett., 67, 18, 2962, (Nov. 1991), BNL 46714.

Hastings, J.B., Siddons, D.P., vanBurck, U., Hollatz, R., Bergmann, U., "Mössbauer Spectroscopy Using Synchrotron Radia" tion", Phys, Rev. Lett., 66, 770 (1991), BNL, 45302.

Hastings, J. B., (NSLS Department), "Performance and Upgrades of the NSLS Storage Rings and Photon Sources", Rev, Sci. Instr., 63 (1), 1565-1566, (1992), BNL 46810. 
Heese, R., Kalsi, S., Leung, E., "The DARPA Compact Superconducting X-Ray Lithography Source Features", Conference Record of the 1991 IEEE Particle Accelerator Conference, Vol, 4, May 6-9, 1991, pp. 2652-64, (1991), BNL 46178.

Hirschmugl, C. J., Williams, G, P., Hoffmann, F. M., Chabal, Y. J., "Adsorbate-Substrate Resonant Interactions Observed for $\mathrm{CO}$ on $\mathrm{Cu}(100)$ and (111) in the Far.IR Using Synchrotron Radiation", J. of Elec. Spec. \& Relat, Phenomena, 54/55, 109, (1990), BNL 45253.

Hirschmugl, C. J., Sagurton, M., Williams, G. P., "Multiparticle Coherence Calculations for Synchrotron Radlation Emission", Phys, Rev. A, 44, 1316, (1991), BNL 46117.

Hsieh, H., Blumberg, R., Heese, R., Murphy, J., Pjerov, S., Sharma, S., Leung, E. M. W., Kalsl, S., Reusch, M. F." "Superconducting Magnet Program for X-Ray Lithography Source at Brookhaven National Laboratory", Conference Rer'ord of the 1991 IEEE Particle Accelerator Conference, May 6-9, 1991 CH3038-7, Vol, 4, p. 2248-50, (1991), BNI, 46475.

Hulbert, S. L., "Interaction of InGa Liquid Alloy Coolant with Gold Coated Optical Materials", Rev. Soi. Instr, 63, 505, (1992), BNL 46496.

Hulbert, S. L., Kao, C. C., Garrett, R, F., Bartynskl, R.A., Yang, S., Weinert, M., Jensen, E., Zehner, D. M., "A Comparison of the Surface Electronic Structure of $\mathrm{Ta}(100)$ and $\mathrm{TaC}(111)$ Using Auger-Photoelectron Coincidence Spectroscopy (APECS)", J. Vac. Sci. Tech., A9, 1919 (1991), BNL 45325.

Jain, A., Ben-Zvl, I., Paul, P., Wang, H., Lombardi, A., "Status of the SUNY Superconducting RFQ", May 6-9, 1991, Conference Record of the IEEE Particle Accelerator Conference, Vol. 4, pp. 2444-46, (1991), BNL 46642.

Johnson, E. D., Kao, C. C. Hastings, J. B., "Recent Advances in Soft X-Ray Scattering Instrumentation", Rev. Sci. Instrum., 63 (1), 1443-46, (1992), BNL 46648.

Johnson, E. D., Fauchet, A. M., Zhang, X., "Correlation of Photon Beam Motion with Vacuum Chamber Cooling on the NSLS X-Ray Ring", Rev. Sci. Instrum., 63, (1), 513-18, (1992), BNL 46838.

Johnson, E, D., Freeman, J., Powell, F., "Compact UHV Valve with Field Replaceable Windows", Rev. Sci. Instrum., 63, (1), 1282-84, (1992), BNL 46493.

Johnson, P. D., Hulbert, S. L., "Inverse Photoemisslon", Rev. Soi. Instrum., 61 (9), 2277 (1990), BNL 43422.

Kao, C.-C., Merrill, R. P., "A Simple Model for the Calculation of the Extra-atomic Relaxation Energies in Ionic Solids", $J$, Phys, and Chem. Solids, Vol. 52, No. 7, 909-912, (1991), BNL 46928.

Kao, C.-C., Johnson, E. D., Hastings, J. B., Siddons, D. P., "Soft X-Ray Resonant Magnetic Scattering Study of Thin Films and Multilayers", SPIE Proceedings, "Production Analysis of Polarized X-Rays", Editor D. P. Siddons, Vol, 1548, 149, (1991), BNL 46640.

Karlin, B. A., Cowan, P. L., Woicik, J, C., "X-Ray, Soft X-Ray, and VUV Beam Position Monitor", Rev, Sci. Instrum., 63 (1), 626-529 (1992), BNL 46694.

Kalsi, S., Heese, R., "Calculation of Eddy-currents Induced in a Compact Synchrotron Superconducting Magnet Structure During a Current Ramp", Conference Record of the 1991 IEEE Particle Accelerator Conference, May 6-9, 1991, Vol, 4, pp. 2658-60, (1991), BNL 46176.

Krinsky, S., "Physics and 'Technology Challenges of Ultra Low Emittance Synchrotron Light Sources", Conference Record of the 1991 IEEE Particle Accelerator Conference, May 6-9, 1991, Vol. 1, pp. 11-15, (1991), BNL 46206.

Krishnaswamy, J., Kalsi, S., Hsieh, H., "Magnetic Measurements of the 12-Pole Trim Magnets for the $200 \mathrm{MeV}$ Compact Synchrotron XLS at the National Synchrotron Light Source", Conference Record of the 1991 IEEE Particle Accelerator Conference, May 6-9, 1991, Vol. 4, pp. 2119-21, (1991), BNL 46177.

McWhan, D. B., Hastings, J. B., Kao, C.-C., Siddons, D. P., "Resonant and Non-Resonant Magnetic Scattering", Rev, Sci. Instrum., 63 (1), 1404-08, (1992), BNL 46822.

Moeller, K. D., Siddons, D. P., Hirschmugl, C. J., Scardino, D., Petrone, P., Carlson, D., Williams, G. P., "Two Mirror Wavefront Dividing Interferometer for Infrared Synchrotron Radiation", Appl. Optics, 30, 4297, (1991), BNL 45526.

Murphy, J, B., Biscardi, R., Bittner, J., Blumberg, L. N., Bozoki, E., Desmond, E., Halama, H., Heese, R., Hsieh, H., Keane, J., Kramer, S., Nawrocky, R., Romano, T., Rothman, J., Schuchman, J., Thomas, M., Wang, J, M., "Commissioning of the Phase I Superconducting X-Ray Lithography Source (SXLS) at BNL", Conference Record of the 1991 IEEE Particle Accel. erator Conference, May 6-9,1991, 91CH3038-7, p.1107, (1991), BNL 46183.

Murphy, J. B., Williams, G. P., "Electron Storage Rings as X-Ray Lithography Sources: An Update", Synchrotron Rad. News, Vol, 3, No, 6, p. 18, (1990), BNL 46507.

Nawrocky, R. J., Biscardi, R., Dabrowskl, J., Flannigan, J., Ramamoorthy, S., Rothman, J., Smith, J., So, I., Thomas, M., Decker G., "An Active Interlock System for the NSLS X-Ray Ring Insertion Devices", Conference Record of the 1991 IEEE Particle Accelerator Conference, May 6-9, 1991, Vol, 5, pp. 2790-92, (1391), BNL 46207. 
Oversluizen, 'T, Stoeber, W., Johnson, E. D., "Kinematic Mounting Systems for NSLS Beamlines and Experimentg", Rev, Sot Instru., 63 (1), 1286, (1992) BNL 46494.

Plvit, E., Hanna, S. M., Keane, J "Fast Ferrite Tuner for the BNL Synchrotron Light Source", Conference Record of the IEEE Particle Accelerator Conference, May 6-9, 1991, Cat, 91CH3038-7, Vol, 2, pp. 774-76 (1991), BNL 46182.

Rogers, J. T, Gray, A., Warren, J, B., "A High Repetition Rate Beam Profile Monitor", Workshop on Advanced Beam Instrumentation, 4/22-24/91, Tsukuba, Japan, Vol, 1, p. 141-144, BNL 46695, September 1891.

Romano, T. J., Heese, R., "The Ultra-Fast Injection Kicker for SXLS", Conference Record of the 1901 IEEE Particle Accelerator Conference, May 6-9, 1991, Vol. 2, p. 993-95, (1991), BNL 46476.

Romero, D. B., Carr, G. L., Tanner, D. B., Forro, L., Mandrus, D., Mihaly, L., Williams, G, P." "12k ${ }_{\mathrm{B}} \mathrm{T}_{\mathrm{c}}$ Optical Signature of Superconductivity in Single-Domain $\mathrm{Bl}_{2} \mathrm{Sr}_{2} \mathrm{CaCu}_{2} \mathrm{O}_{8}{ }^{\prime \prime}$, Phys, Rev, B, 44, 2818, (1991), BNL 45118.

Rose, J., Biscardl, R., Broome, W., D'Alsace, R., Keane, J., Wang, J, M., "Dlagnosis and Cure of a Transverse Instability in the NSLS VUV Ring", Conference Record of the 1991 IEEE Particle Accelerator Conference, May 6-9, 1991, Vol, 3, pp. 179496, (1991), BNL 46175.

Schuchman, J, C., Chou, T. S., Halama, H, Hsieh, H., Kim, T., PJerov, S., Stalcu, F, "SXLS Phase II Vacuum System", Conference Record of the 1991 IEEE Particle Accelerator Conference, May 6-9, 1991, 91CH3038-7, Vol. 4, p. 2316, (1991), BNL 46460 .

Shu, D., Warwick, T., Johnson, E. D., "Diagnostic Phosphors for Photon Beams at the ALS and APS", Rev. Sci. Instrum., 63 (1), 548-49, (1992), BNL 46649 .

Singh, O., Nawrocky, R, Flannigan, J., "Automatic Local Beam Steering Systems for NSLS X.Ray Storage Ring - Design and Implementation", Conference Record of the IEEE Particle Accelerator Accelerator Conference, May 6-9, 1991, Vol. 3, pp. 1'628-30, (1991), BNL 46563.

Solomon, L., Galayda, J., Sylvester, C., "Magnetic Measurements of the SXIS Magnets", May 6-9, 1991, Conference Record of the IEEE Particle Accelerator Conference, Vol, 4, pp. 2116-18, (1991), BNL 45567.

Stefan, P. M., Solomon, L, Krinsky, S., Rakowsky, G, "NSLS Prototype Small-Gap Undulator (PSGU)", Conference Record of the 1991 IEEE Particle Accelerator Conference, Vol, 2, pp. 1096-1098, (1991), BNL 45670.

Stojanoff, V., Hämäläinen, K., Siddons, D. P., Hastings, J. B., Berman, L. E., Cramer, S. P., Smith, G., "A High Resolution X-Ray Fluorescence Spectrometer for Near Edge Absorption Studies", Rev. Soi. Instrum., 63 (1), 1125-27, (1992), BNL 46809.

Suorttl, P., Thomlinson, W., Chapman, D., Gmür, N., Greene, R., Lazarz, N., "Performance Evaluation of a Bent Laue Monochromator", Nucl. Instru. \& Meth. in Phys. Res., A297, 268-274, (1990), BNL 43998.

Sutton, M., Mochrie, S. G. J., Greytak, 'T., Nagler, S. E., Berman, L. E., Held, G. A., Stephenson, G. B., "Observation of Speckle by Diffraction with Coherent X-Rays", Nature, V 352, 608, (1991), BNL 46506.

Thomlinson, W., Gmür, N., Chapman, D., Garrett, R., Lazarz, N., Morrison, J., Reiser, P., Padmanabhan, V., Ong., L., Green, S., Thompson, A., Zeman, H., Hofstadter, R., Brown, G., Glacomini, J., Gordon, I1., Rubenstein, E., "Venous Synchrotron Coronary Angiography", The Lanoet, Vol. 397, February 9, 1991, 360, BNL 46030.

Thomlinson, W., Gmür, N., Chapman, D., Lazarz, N., Moulin, H., Garrett, R., Thompson, A., Zeman, H., Brown, G, Morrison, J., Reiser, P., Padmanabahn, V., Ong, L., Green, S., Glacomini, J., Gordon, H., Rubenstein, E., "First Operation of the Medical Research Facility at the NSLS F'or Coronary Anglography", Rev, of Sci, Instr, 63 (1), 625-628 (1992), BNL 46109.

Tourillon, G., Guay, D., Fontaine, A., Garrett, R., Williams, G. P., "Characterization of Metal/Organic Molecule and Metal/ Polymer Interfaces by NEXAFS Spectroscopy", Faraday Discuss, Chem. Soc, 89, 275-290, BNL 46609, September 1991.

van Steenbergen, A., Gallardo, J., Romano, T., Woodle, M., "Fast Excitation Variable Period Wiggler", Conference Record of the 1991 IEEE Particle Accolerator Conference, May 6-9, 1991, Vol. 5, pp. 2724-26, (1991), BNL 45564.

Wang, H., Ben-Zvi, I., Jain, A., Paul, P., Lombardi, A., "Numerical Simulation of a Short RFQ Resonator Using the MAFI.A Codes", Conference Record of the 1991 IEEE Particle Accelerator Conference, May 6-9, 1991, Vol. 5, pp. 3038-40, (1991), BNL 46640 .

Warwick, T., Shu, D., Rodricks, B., Johnson, E. D., "Prototype Photon Position Monitors for Undulator Beams at the Advanced Light Source", Rev. Sci. Instrum., 63 (1), 550-53, (1992), BNL 46743.

Williams, G. P., "Infrared Synchrotron Radiation Instrumentation and Applications", Rev, Sci. Instrum. 63 (1), 1535, (1992), BNL 46592 .

Williams, G. P., Winick, H., "Ovorview of Synchrotron Radiation Sources World-Wide", Synchrotron Rad. News, Vol 4, No, 5, 23, (1991), BNL 46508 .

Yu, L. H., "Generation of Intense UV Radiation by Subharmonically Seeded Single Pass FEL", Phys, Rev, A, Vol. 44, No, 8, 5178-93, BNL 45970. 
Yu, L, H, Biscardl, R., Bittner, J, Fauchet, A. M., Krinsky, S, Nawrocky, R, J, Rothman, J, Singh, O, V., Yang, K, M.1 "Real Time Global Orbit Feedbac System for the NSLS X-Ray Ring", Conference Record of the 1991 IEEE Particle Accel. erator Conference, Vol, 4, May 6 -9, 1991, pp, 2542-44, (1991), BNL 45565.

Yu, L, H., Krinsky, S, Gluckstern, R. L., "Universal Scaling Function for FEL Gain", Submitted to the Twelfth International Free Electron Laser Conference, Paris, France, September 17-21, 1990, BNL 46152, November 1890.

Zhang, X., Murphy, J. B., Krinsky, S. "Undulator Based Synchrotron Radiation Source in the E-30 eV Spectral Region", Conference Recurd of the 1991 IEEE Particle Accelerator Conference, May 6-9, 1991, Vol, 5, pp, 2676-78, (1991), BNL 46185, (Abstract BNL 45668).

Zeman, H. D., Dilmanian, F. A, Garrett, R. F, Berman, L. E, Chapman, L, D., Hastings, J. B., Oversluizen, T, Siddons, D. P. Stojanoff, V. Thomlinson, W. C. "An X-Ray Monochromator for Dual-Energy Computerized Tomography Using Synchrotion Radiation", Nucl. Instr, \& Meth, in Phys, Res, B56/57, 1218-1222, (1991), BNL 46693.

Zeman, H. D., DiBlanca, F, A., Thomlinson, W, C., "A Kinestatlc Charge Detector for Intravenous Coronary Angiography Using Synchrotron Radiation X.Rays", IEEE Trans. Nucl. Sco, Vol. 38, No. 2, April 1991, 641-67, BNL 46692. 


\section{Preprints of the NSLS Staff}

Barklgla, K. M., Fajer, J, Furenlid, L. R., Renner, M. W. and Thompson, M, A., "Porphyrin Chemistry". Abstract, 15th DOE Solar Photochemistry Research Conference, Snowmass CO, June, 1991. BNL 45906.

Barkigia, K, M., Furenlid, L, R, Medforth, C, J, Renner, M. W., Smith, K, M., and Fajer, J., "Crystallographic and EXAFS Studies of Sterically Crowded Porphyrins". Presented at the 4th North American Chemical Congress, NY, Aug. 25-30, 1991. BNL 46099 .

Batchelor, K., Ben-Zvi, I, Fernow, R, C., Fischer, J, Flsher, A, S., Gallardo, J., Ingold, G, Kirk, H, G, Leung, K.P., Malone, R, Pogorelsky, I, Srinivasan-Rao, TT, Rogers, J., Tsang, T., Sheehan, J., Ulc, S., Woodle, M., Xle, J., Zhang, R. S., Lin, L, Y., McDonald, K. T, Russell, D. P., Hung, C. M., Wang, X. J." "Performance of the Brookhaven Photocathode RF Gun", 1991 International FEL Conference, Santa $\mathrm{Fe}, \mathrm{NM}, 8 / 26-30 / 91$, BNL 46679, September 1991.

Batchelor, K., Ben-Zvi, I., Fernow, R, C., Fisher, A. S., Friedman, A., Gallardo, J., Ingold, G., Kirk, H., Kramer, S., Lin, L., Rogers, J. T', Sheehan, J. F., vanSteenbergen, A., Woodle, M., Xie, J., Yu, L. H., Zhang, R., Bhowmik, A., "Status of the Visible Free-Electron Laser at the Brookhaven Accelerator Test Facillty", 1991 Internatlonal FEL Conference, Santa Fe, NM, 8/26-30/91, BNL 46681, September 1991.

Ben-Zvi, I, DiMauro, L. F., Krinsky, S., White, M. G., Yu, L. H., Batchelor, K., Friedman, A., Fisher, A. S., Halama, H., Ingold, G., Johnson, E. D., Kramer, S., Rogers, J. T., Solomon, L., Wachtel, J., Zhang, X., "Proposed UV.FEL User Facility at BNL". 1991 International FEL Conference, Santa Fe, NM, 8/26-30/91, BNL 466'73, September 1991.

Ben-Zvl, I, Friedman, A., Hung, C.M., Ingold, G., Krinsky, S., Yang, K. M., Yu. L. H., Lehrman, I, Welssenburger, D., "Design of a Harmonic Generation FEL Experiment at BNL". 1991 International FEL Conference, Santa Fe, NM, 8/26-30/91, BNL 46682, September 1991.

Ben-Zvi, I., Yang, K. M., Yu, L. H., "The 'Fresh-Bunch' Technique in FELS". 1991 International FEL Conference, Santa Fe, NM, 8/26-30/91, BNL 46688, September 1991.

Ben-Zvi, I., Fernow, R., Gallardo, J., Ingold, G., Sampson, W., Woodle, M., "Theoretical Analysis of Iron-Bore Superconducting Undulators", 1991 International FEL Conference, Santa Fe, NM, 8/26-30/91, BNL 46702, September 1991.

Ben-Zvi, I., Fernow, R., Gallardo, J., Ingold, G., Sampson, W., Woodle, M., "Performance of a Superconducting, High Field Subcentimeter Undulator". 1991 International FEL Conference, Santa Fe, NM, 8/26-30/91, BNL 46713, September 1991.

Bhowmik, A., Lordi, N., Ben-Zvi, I., Gallardo, J., "Resonator Design for a Visible Wavelength Free Electron Laser". International Conference on Lasers, San Diego, CA, BNL 46644, September 1991.

Fajer, J., Barkigia, K. M., Furenlid, L. R., Smith, K. M., and Renner, M. W., "Structural and Electronic Control of Reaction Sites and Reactivity in Hydroporphyrins". Abstract, 13th International Congress of Heterocyclic Chemistry, Corvallis, OR, August, 1991. BNL 46069.

Foerster, C. L., Korn, G., "The Search for Low Photodesorption Coatings". Submitted to the Vacuum Design of Synchrotron Light Sources Conference, ANL, 11/13-15/91, BNL 46492, July 1991.

Halama, H., "NSLS Vacuum System Operating Experience Conditioning and Desorption Yields, Vacuum Design of Synchro. tron Light Sources", 11/13-15/91, ANL, IL, BNL 46472, August 1991.

Hämäläinen, K., Kao, C. C., Hastings, J, B., Siddons, D. P., Berman, L. E., Stojanoff, V., Cramer, S. P." "Spin-Dependent $\mathrm{X}$-Ray Absorption of $\mathrm{MnO}$ and $\mathrm{MnF}_{2}{ }^{\prime \prime}$, Phys. Rev. Lett., BNL 46651, September 1991.

Hastings, J. B., Chapman, D., Siddons, D. P., Grover, J, R., "Nuclear Fluorescence Using SR Excitation", Abstract submitted to the 1991 Particle Accelerator Conference, 5/6-9/91, San Francisco, CA, BNL 45611A, December 1990.

Jensen, E., Bartynski, R. A., Garrett, R. F., Hulbert, S. L, Johnson, E. D., Kao, C.-C., "Origin of the Low Energy Tail in the Al L $\mathrm{L}_{2,3}$ VV Auger Spectrun Studied with Auger Photoelecytron Colncidence Spectroscopy", Submitted to Phys. Rev, B., November, 1990.

Johnson, P. D., Hulbert, S. L., Klaffky, R, Brookes, N. B., Clarke, A., Sinkovic, B., Smith, N. V., Celotta, R., Kelly, M. H., Pierce, D. T., Scheinfein, M. R., Waclawski, B. J, and Howells, M. R., "Spin-Polarized Photoemission Spectroscopy of Magnetic Surfaces Using Undulator Radiation". Submitted to Review of Sclence Instruments, BNL. 47015.

Joksch, S., Graeff, W., Hastings, J. B, Siddons, D. P., "Performance of an X-ray Optical Time Delay Line with Synchrotron Radiation". Review of Sclentific Instruments, BNL 46575, September 1991.

Laster, B. H., Thomlinson, W. C., Fairchild, R. G., "Blological Efficacy of the Auger Effect in Iodine", Radiation Research (July 1991).

Moeller, K. D., Williams, G. P., Steinhauser, S., Hirschmugl, C., Smith, J, C., "Hydration-Dependent Far-Infrared Absorption in Lysozume Detected Using Synchrotron Radiation". Submitted to Biophysical Journal, BNL 46574, September 1991. 

Renner, M. W., Furenlid, L. R., and Fajer, J., "Structural and Spectroscopic Studies of Ni(II) and Ni(I) Hydroporphyrins as Models for F430". Presented at the 3rd Symposium on Inorganic Biochemistry and Molecular Biophysics, Karparz, Poland, September, 1991. BNL 45988.

Russell, Andrea E., Williams, G. P., O'Grady, William E., "In Situ Far-Infrared Studies of the Electrode/Electrolyte Interface: Chloride and Water Adsorbed on Ag(111)". Physical Review Letters, BNL 46516. August 1991.

Schuchman, J. C., "Review of Vacuum Systems for X-ray Lithography Light Sources", 37th National Symposium of the American Vacuum Society, 10/8-12/90, Toronto, Canada, BNL 46470, August 1991.

Yu, L. H., Krinsky, S. Friedman, A., Gluckstern, R. L., van Zeijts, J. B. J., "Reduction of FEL Gain Due to Wiggler Errors", Submitted to 1991 Intl. FEL, Conference, Santa Fe, NM, 8/26-30/91, BNL 46582, August, 1991.

Yu, L. H., Krinsky, S., Gluckstern, R. L., van Zeijts, J. B. J., "The Effect of Wiggler Errors on FEL Gain". Phys. Rev. A, BNL 46608, September 1991. 


\section{BNL Formal and Informal Reports of the NSLS Staff}

Bozoki, E., Transport Line From the LINAC to the SXLS Ring. Informal Report, BNL 46249, May 1991.

Gmur, N. F., (Ed.) Imaging Research at the National Synchrotron Light Source. Informal Report, BNL 45765 (reprinted from BNL 52274), July 1991.

Gmur, N., National Synchrotron Light Source Community Directory, Third Edition. Informal Report, BNL 45197, October 1990.

Gmur, N. F., National Synchrotron Light Source User's Manual: Guide to the VUV and X-ray Beamlines - Fourth Edition. Informal Report, BNL 45764, February 1991.

Heese, V., Gmur, N., Thomlinson, W., A Survey of Medical Diagnostic Imaging Technologies. Informal Report, BNL 46739, September 1991.

Hulbert, S. L., Lazarz, N., National Synchrotron Light Source Annual Report 1990. BNL 52272 (1991).

Johnson, E. D., NSLS Process Water Systems: Description of Physical Plant and Retrospective Analysis of Fault Reports. NSLS Informal Report, BNL 46116, June 1991.

Krinsky, S., Bittner, J., Fauchet, A. M., Johnson, E. D., Keane, J., Murphy, J., Nawrocky, R. J., Rogers, J., Singh, O. V., Yu, L. H., Storage Ring Development at the National Synchrotron Light Source. NSLS Informal Report, BNL 46615, September 1991.

Solomon, L., Galayda, J., Magnetic Measurements of the X25 and X21 Wigglers at the NSLS. Informal Report, BNL 45665, January 199 . 

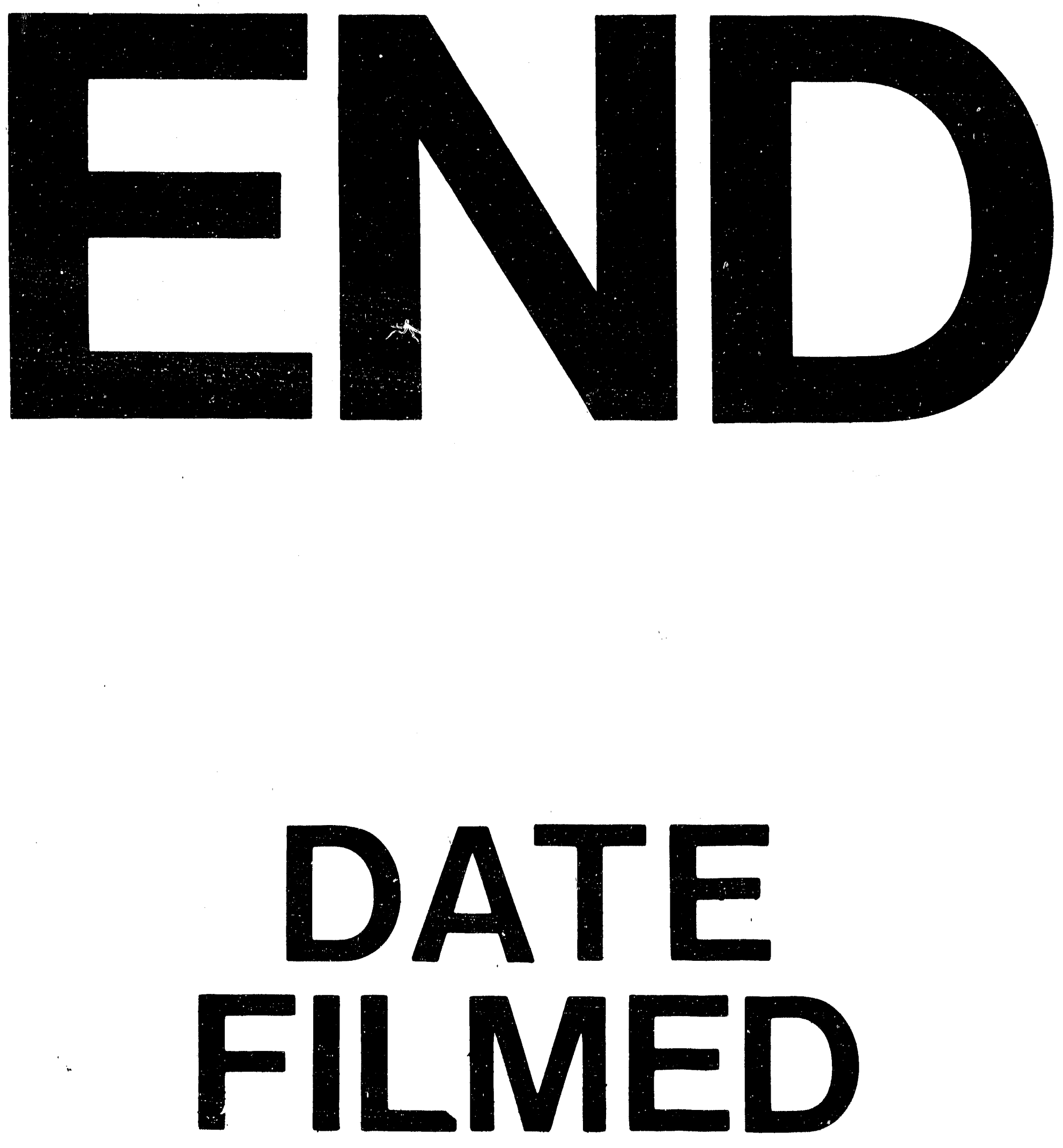

$\neq$

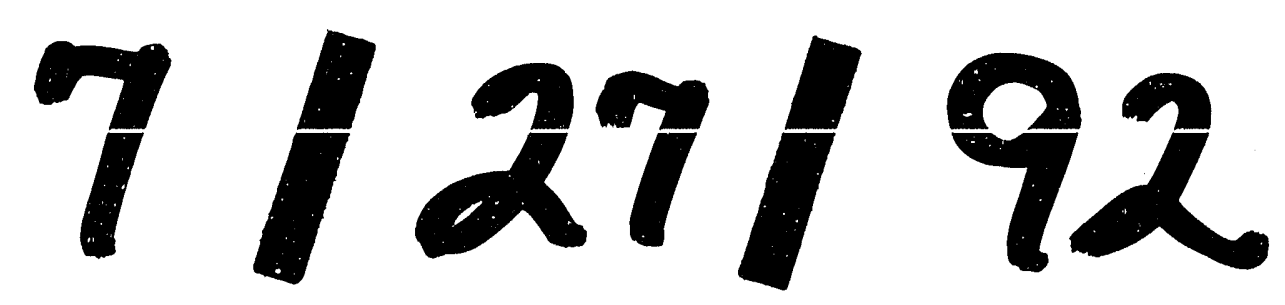


